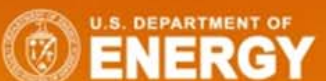

Office of Electricity Delivery and Energy Reliability (OE1)

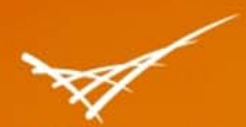

Pacific Northwest

NATIONAL LABORATORY

Proudly Operated by Battelle Since 1965

\title{
National Assessment of Energy Storage for Grid Balancing and Arbitrage: Phase 1, WECC
}

M Kintner-Meyer

$\mathrm{P}$ Balducci

W Colella

M Elizondo

C Jin

T Nguyen

$\checkmark$ Viswanathan

Y Zhang

June 2012 


\title{
DISCLAIMER
}

This report was prepared as an account of work sponsored by an agency of the United States Government. Neither the United States Government nor any agency thereof, nor Battelle Memorial Institute, nor any of their employees, makes any warranty, express or implied, or assumes any legal liability or responsibility for the accuracy, completeness, or usefulness of any information, apparatus, product, or process disclosed, or represents that its use would not infringe privately owned rights. Reference herein to any specific commercial product, process, or service by trade name, trademark, manufacturer, or otherwise does not necessarily constitute or imply its endorsement, recommendation, or favoring by the United States Government or any agency thereof, or Battelle Memorial Institute. The views and opinions of authors expressed herein do not necessarily state or reflect those of the United States Government or any agency thereof.

\author{
PACIFIC NORTHWEST NATIONAL LABORATORY \\ operated by \\ BATTELLE \\ for the \\ UNITED STATES DEPARTMENT OF ENERGY \\ under Contract DE-AC05-76RL01830
}

Printed in the United States of America

Available to DOE and DOE contractors from the

Office of Scientific and Technical Information,

P.O. Box 62, Oak Ridge, TN 37831-0062;

ph: (865) 576-8401

fax: $(865) 576-5728$

email: reports@adonis.osti.gov

\begin{abstract}
Available to the public from the National Technical Information Service, U.S. Department of Commerce, 5285 Port Royal Rd., Springfield, VA 22161 ph: (800) 553-6847 fax: (703) 605-6900 email: orders@ntis.fedworld.gov

online ordering: http://www.ntis.gov/ordering.htm
\end{abstract}




\title{
National Assessment of Energy Storage for Grid Balancing and Arbitrage: Phase 1, WECC
}

\author{
M Kintner-Meyer \\ P Balducci \\ W Colella \\ M Elizondo \\ C Jin \\ T Nguyen \\ V Viswanathan \\ Y Zhang
}

June 2012

Prepared for

the U.S. Department of Energy

under Contract DE-AC05-76RL01830

Funded by the Energy Storage Systems Program of the U.S. Department of Energy

Dr. Imre Gyuk, Program Manager

Pacific Northwest National Laboratory

Richland, Washington 99352 



\section{Executive Summary}

Electricity production from wind and other renewables technology has increased significantly to meet the renewable portfolio standards (RPS) targets imposed by 31 U.S. states and the District of Columbia. Energy storage is attracting greater interest as an enabling technology for integrating variable renewable power into the electric grid, addressing grid reliability challenges, and increasing overall infrastructure utilization. The integration of renewable energy technology into the U.S. grid is one of the key drivers for the growing interest in stationary energy storage systems. Other countries are also interested in advanced energy storage systems for accommodating the variable nature of renewable resources and the inherent uncertainty in accurately forecasting production. Internationally, significant investments in research and development for advanced energy storage systems are being made to address the perceived need that energy storage will be an important component of the future power grids worldwide.

\section{Motivation for the National Assessment}

To provide a better understanding to industry, this National Assessment of Energy Storage for Grid Balancing and Arbitrage attempts to estimate the market size for stationary energy storage systems for two specific applications: 1) balancing services necessary to accommodate the growing variations in the generation supply from renewable energy resources, and 2) energy arbitrage that provides congestion management strategies and the potential to lower the cost of delivering electricity. There are many other benefits and values that energy storage can furnish to grid operations. Earler reports identified a total of 17 applications, in which electric energy storage could provide benefits and value to both end-use customers and the electric grid. The applications not addressed here are are either location-specific or difficult to assess without detailed grid modeling capability requiring highly detailed data. To initiate the discussion on the potential market size of grid-connected energy storage that could be plausibly and defensibly integrated into the grid (and considering competing technologies that vie for the same market share and market opportunities of energy storage) a balance was struck. This balance means addressing fewer storage applications, however, for the entire U.S. grid, rather than a set of highly detailed case studies with limited regional scope. Furthermore, significant fundamental work will still need to be done to estimate multiple values of energy storage in a comprehensive manner that avoids double-counting of benefits. Clearly, the market for grid energy storage is expected to be significantly larger than might be estimated solely from this study.

This assessment will ultimately be performed for the entire U.S. grid. Because of regional differences in the distribution of renewable resources and the structural differences in the transmission and generation mix, the analysis was performed on a regional basis using the North American Electric Reliability Corporation (NERC) 22 sub-regions. This document is the first of two reports that comprise the entire National Assessment of Grid-Connected Energy Storage. Volume I discusses Phase I of the National Assessment. It includes the western grid under the Western Electricity Coordinating Council jurisdiction. Volume II, to be published later, will include the results for the Eastern Interconnect and the Electric Reliability Council of Texas (ERCOT). The regional disaggregation of the U.S. grid is shown in Figure ES.1. 


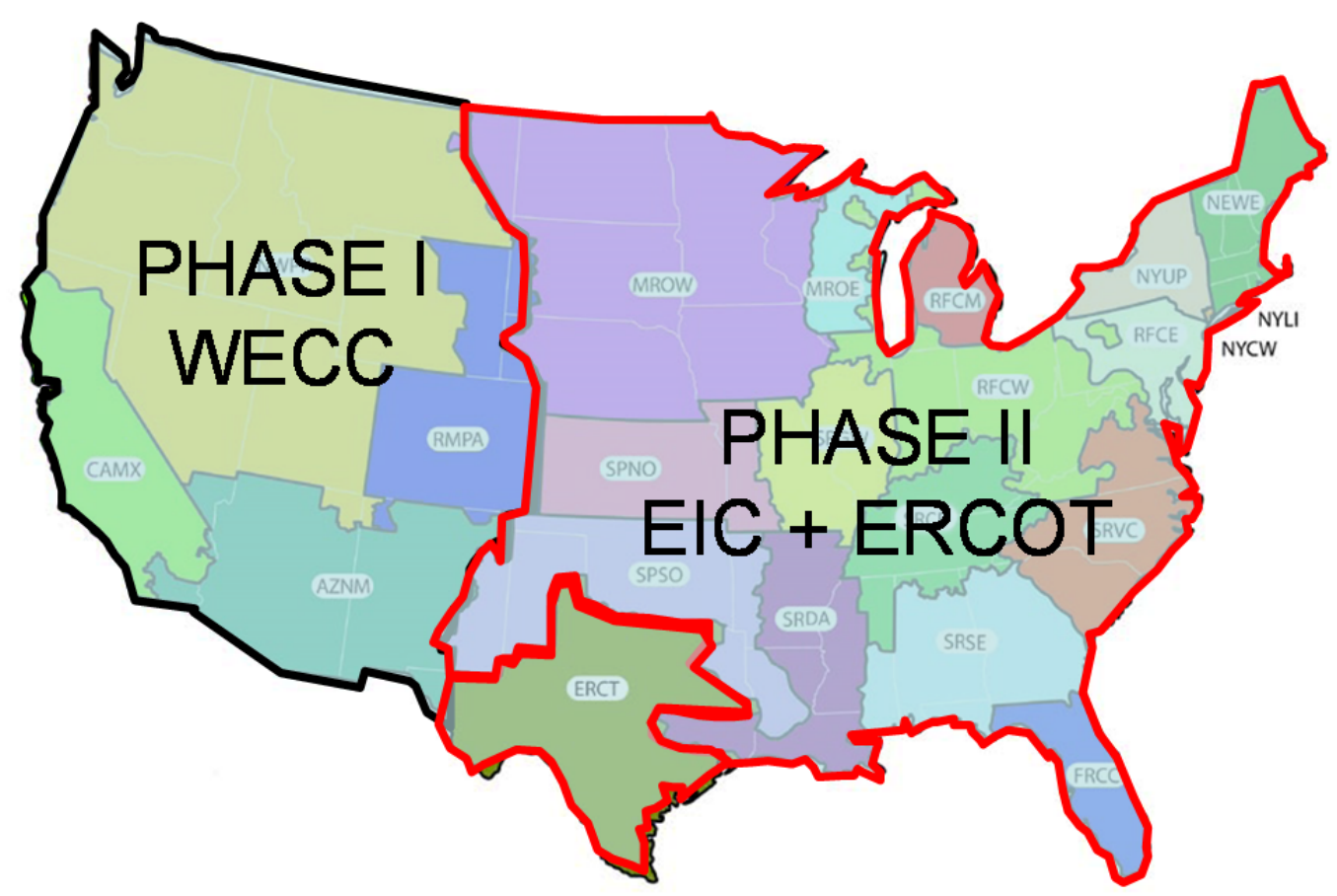

Figure ES.1. Regional Resolution of the National Assessment

\section{Key Questions Addressed}

This assessment addresses the following questions:

1. What are the future balancing requirements ${ }^{1}$ necessary to accommodate enhanced wind generation capacity, so as to meet the RPS of about $20 \%$ of the generation for the Western Electricity Coordinating Council (WECC) in 2020? This analysis assumes that state-specific RPS above 20\%, such as California's 33\% RPS target for 2020, will be honored. Estimates are derived and discussed for four WECC sub-regions. These sub-regions are the Arizona-New Mexico-Southern Nevada (AZNM) Power Area, California-Mexico (CAMX) Power Area, Northwest Power Pool (NWPP), and Rocky Mountain Power Area (RMPA). From a market size perspective it is insightful to estimate both the additional balancing requirement between 2010 and a 2020 grid scenario as well as the total balancing requirements for the 2020 grid scenario. The additional requirements estimate the new demand of balancing services. The total requirement includes replacement options for storage to displace existing generators providing this service.

\footnotetext{
${ }^{1}$ A balancing market is a niche market within a competitive electricity market for last-minute, just-in-time, rapidresponse electricity. This market may demand either increases or decreases in a quantity of electric power. Electricity generators are paid to quickly ramp up or ramp down their electric power in this market. This market results from discrepancies between scheduled electric power generation and actual real-time electric demand and generation. This market is often served by fast-ramping electric power plants like gas turbines and by demand response.
} 
2. What are the most cost-effective technology options for providing additional balancing requirements today and in 2020 assuming technological progress? Our analysis includes the following technologies:
i. Combustion turbine as a base case technology
ii. Na-S (Sodium Sulfur) batteries
iii. Li-ion (Lithium-ion batteries)
iv. Flywheel
v. CAES (Compressed Air Energy Storage)
vi. Redox Flow batteries
vii. Pumped Hydroelectric (PHES) Storage
viii. Demand Response
ix. Hybrid energy storage systems (configurations of various above mentioned storage technologies)

3. What is the market size (quantified in MW and MWh) for energy storage and its respective cost targets (expressed in $\$ / \mathrm{kWh}$ ) for balancing and energy arbitrage applications by regions?

\section{Key Outcomes}

Pacific Northwest National Laboratory (PNNL) analyzed a hypothetical 2020 grid scenario in which additional wind power is assumed to be built to meet WECC's 20\% renewable energy portfolio standard target. Several models were used to address the three questions, including a stochastic model for estimating the balancing requirements using current and future wind statistics and the statistics of forecasting errors. A detailed engineering model was used to analyze the dispatch of energy storage and fast-ramping generation devices for estimating capacity requirements of energy storage and generation that meet the new balancing requirements. Financial models estimated the life-cycle cost (LCC) of storage and generation systems and included optimal sizing of energy storage and generation to minimize LCC. Finally, a complex utility-grade production cost model was used to perform security constrained unit commitment and optimal power flow for the WECC.

\section{Outcome 1: Total Balancing Market in the WECC is Estimated to be $6.32 \mathrm{GW}$ Assuming about 24 GW of Installed Wind Capacity in 2020}

The total amount of power capacity for a 20\% RPS scenario in the WECC for 2020 would require a total intra-hour balancing capacity of approximately $\sim 6.32 \mathrm{GW}$. The total market size was estimated for the WECC by NERC sub-regions based on the potential for energy storage in the high-value balancing market. The energy capacity, if provided by energy storage, would be approximately $2.0 \mathrm{GWh}$, or a storage that could provide power at rated capacity for about 20 minutes. The additional intra-hour balancing capacity that is required to accommodate the variability due to capacity addition in wind technology and load growth from 2011-2020 was estimated to be $1.53 \mathrm{GW}$. If these additional balancing services were provided by new energy storage technology, the energy capacity would be about $0.58 \mathrm{GWh}$, or a storage capable of providing electricity at the rated power capacity for about 20 minutes. 
The regional distribution of balancing requirements within the WECC is driven by load forecasting wind prediction errors. Because of the non-homogeneous distribution of the loads and wind across the WECC region, the balancing requirements increase with load and wind capacity. NWPP and California were the two major regions with significant intra-hour balancing requirements.

Model results indicate that the new balancing requirements will span a spectrum of variability, from minute-to-minute variability (intra-hour balancing) to those indicating cycles over several hours (interhour balancing). This study focused on the intra-hour balancing needs as they include sharp ramp rates that are of significant concern to grid operators. Furthermore, a single-balancing area for each of the 4 WECC sub-regions was assumed, which tends to under-estimate both the magnitude and the variability in the balancing market relative to current conditions. ${ }^{1}$ As a result, it is reasonable to infer that the analysis shown here may under-estimate required levels of storage or generation needed to serve the balancing market. The additional and total intra-hour balancing requirements are presented in Table ES.1 for the four consolidated balancing areas.

Table ES.1. Additional and Total Intra-Hour Balancing Requirements for WECC Sub-Regions in 2020 for $20 \%$ RPS

\begin{tabular}{|c|c|c|c|c|c|c|c|}
\hline & $\begin{array}{c}\frac{\text { Additional }}{\text { Balancing }} \\
\text { Power } \\
\text { Required } \\
\text { (MW) }\end{array}$ & $\begin{array}{l}\text { Total } \\
\text { Balancing } \\
\text { Power } \\
\text { Required } \\
\text { (MW) }\end{array}$ & $\begin{array}{c}\text { Total } \\
\text { Balancing } \\
\text { Power } \\
\text { Required as a } \\
\text { Percentage of } \\
\text { Peak Load } \\
\text { (\%) }\end{array}$ & $\begin{array}{c}\text { Marginal } \\
\text { Balancing } \\
\text { Power } \\
\text { Required as a } \\
\text { Percentage } \\
\text { Wind Capacity } \\
\text { (\%) }\end{array}$ & $\begin{array}{l}\text { Existing } \\
\text { Wind } \\
\text { Capacity } \\
\text { (MW) }\end{array}$ & $\begin{array}{l}\text { Additional } \\
\text { Wind } \\
\text { Capacity } \\
\text { (MW) }\end{array}$ & $\begin{array}{c}\text { Total } \\
\text { Wind } \\
\text { Capacity } \\
\text { in 2020 } \\
\text { (MW) }\end{array}$ \\
\hline AZNM & 210 & 1220 & 4 & 22 & 390 & 970 & 1360 \\
\hline CAMX & 530 & 2400 & 4 & 13 & 2430 & 4110 & 6540 \\
\hline NWPP & 280 & 2020 & 3 & 7 & 5560 & 4200 & 9760 \\
\hline RMPA & 510 & 670 & 5 & 10 & 1170 & 5160 & 6330 \\
\hline Total & 1530 & 6320 & & & 9550 & 14440 & 23990 \\
\hline
\end{tabular}

This study concludes that the future total intra-hour balancing requirements to address both load and renewable variability are expected to range between $3 \%$ and $5 \%$ of the peak load in a given region. Furthermore, on the margin for every additional unit of wind capacity power, approximately 0.07 to 0.22 units of intra-hour balancing need to be added.

\footnotetext{
${ }^{1}$ The main factor that contributes to the under-estimation of the balancing reserve is the assumption that sharing the variability of resources and loads across a broader region reduces the per unit variability with a resulting reduction in required reserves. At present, neither the markets nor the operations are aggregated to the degree assumed in this study.
} 


\section{Outcome 2: Each Technology Option Requires its Own Size to Meet the Future Balancing Needs}

The following technology cases were analyzed:

1. Combustion turbines (CT)

2. Na-S (Sodium Sulfur) batteries integrated with combined cycle gas turbine (CCGT)

3. Li-ion (Lithium-ion) batteries integrated with CCGT

4. Flywheels integrated with CCGT

5. CAES integrated with CCGT

6. Redox (reduction-oxidation) flow batteries integrated with CCGT

7. PHES (pumped hydro energy storage) with frequent mode changes per day ${ }^{1}$

8. PHES with two mode changes per day ${ }^{1}$

9. Demand Response technology (only electric vehicle [EV] charging considered).

In technology case 1 , the CTs are used to provide balancing with controlled variable power output. In technology cases 2-8, CCGTs are used to compensate for the storage electricity loss of different types of batteries, flywheels, CAES, and PHES ${ }^{2}$. It should be noted that for the Na-S case an assumption was used that battery systems with a ratio of rated energy to rated power $(\mathrm{E} / \mathrm{P}=1)$ will be available in future, as opposed to the currently available ratio $\mathrm{E} / \mathrm{P}=7$.

Table ES.2 presents the sizing results for both the power and energy requirements for each of the aforementioned nine cases based on the additional intra-hour balancing services. Table ES.3 illustrates the sizing requirements total intra-hour balancing. Capacity requirements are based on a $100 \%$ energy storage depth of discharge (DOD). Under this assumption, the energy capacity of the storage device is fully utilized during each cycle, with the device cycled from a fully charged to a fully discharged state. From a LCC analysis viewpoint, there may be economic benefits to over-sizing the battery such that it is cycled at a DOD of less than $100 \%$ to improve the life of the energy storage device. DOD impacts both battery lifetime and size. In turn, battery sizing influences capital costs. The tradeoff between energy storage cycle life and capital costs are examined in detail later in the report.

\footnotetext{
${ }^{1}$ To bridge the waiting period during the mode changes, a small Na-S battery was assumed.

${ }^{2}$ A source of energy is needed to charge the storage technologies. This energy that flows through the storage technologies is assumed to come from existing generation on the margin. CCGT was assumed to be marginal generation most of the time.
} 
Table ES.2. Power and Energy Requirements by Technology Case to Meet Additional Intra-Hour Balancing Requirements

\begin{tabular}{llcccccccc}
\hline & \multirow{2}{*}{ Technology } & \multicolumn{3}{c}{ AZNM } & \multicolumn{2}{c}{ CAMX } & \multicolumn{2}{c}{ NWPP } & \multicolumn{2}{c}{ RMPA } \\
\cline { 3 - 10 } Cawh & \multicolumn{1}{c}{ GW } & GWh & GW & GWh & GW & GWh & GW & GWh \\
\hline C1 & Combustion turbine & 0.21 & - & 0.53 & - & 0.28 & - & 0.52 & - \\
C2 & Na-S & 0.21 & 0.08 & 0.53 & 0.18 & 0.28 & 0.13 & 0.51 & 0.19 \\
C3 & Li-ion & 0.21 & 0.08 & 0.53 & 0.18 & 0.28 & 0.12 & 0.51 & 0.19 \\
C4 & Flywheel & 0.21 & 0.07 & 0.53 & 0.17 & 0.28 & 0.11 & 0.51 & 0.18 \\
C5 & CAES 2 modes & 0.37 & 1.69 & 0.92 & 6.32 & 0.52 & 2.84 & 0.99 & 6.16 \\
& 7-min waiting period, Na-S & 0.12 & 0.01 & 0.16 & 0.02 & 0.1 & 0.01 & 0.23 & 0.02 \\
C6 & Flow battery & 0.21 & 0.08 & 0.52 & 0.19 & 0.28 & 0.13 & 0.51 & 0.19 \\
C7 & Pumped hydroelectric (PH) & 0.21 & 0.07 & 0.53 & 0.17 & 0.28 & 0.12 & 0.51 & 0.18 \\
& multiple modes & & & & & & & & \\
& 4-min waiting period, Na-S & 0.08 & 0.01 & 0.18 & 0.02 & 0.1 & 0.02 & 0.17 & 0.03 \\
C8 & PH 2 modes & 0.37 & 1.7 & 0.92 & 6.35 & 0.52 & 2.86 & 0.99 & 6.19 \\
& 4-min waiting period, Na-S & 0.07 & 0.01 & 0.1 & 0.01 & 0.07 & 0.01 & 0.18 & 0.01 \\
C9 & DR (demand response) & 0.75 & - & 1.53 & - & 1.01 & - & 1.73 & - \\
\hline
\end{tabular}

The two storage technologies (C5, C8), which require a distinct mode change from charging to discharging, demand significantly higher power capacities than those that can switch instantaneously between charging and discharging. Because the entire balancing requirements (from maximum increment to maximum decrement) must be provided in one mode, the power and the energy capacity of such technologies must be significantly increased. The large power capacity requirement for DR resources is attributable to low resource availability during the early morning, low load conditions, when there are few resources available. To compensate for the low availability of resources, the resources have to be increased. In this particular case, where we assumed that all of the DR resources are provided by EV charging, a significant number of EVs must be engaged to overcome the low load condition in early morning hours when most of the EVs are fully charged.

The results for the total intra-hour requirement are shown in Table ES.3. 
Table ES.3. Power and Energy Requirements by Technology Case to Meet Total Intra-Hour Balancing Requirements

\begin{tabular}{llrrrrrrrr}
\hline & \multirow{2}{*}{ Technology } & \multicolumn{2}{c}{ AZNM } & \multicolumn{2}{c}{ CAMX } & \multicolumn{2}{c}{ NWPP } & \multicolumn{2}{c}{ RMPA } \\
\cline { 3 - 9 } Case & \multicolumn{1}{c}{ GW } & GWh & GW & GWh & GW & GWh & GW & GWh \\
\hline C1 & Combustion turbine & 1.2 & - & 2.3 & - & 2.0 & - & 0.7 & - \\
C2 & Na-S & 1.2 & 0.5 & 2.4 & 0.7 & 2.0 & 0.6 & 0.7 & 0.2 \\
C3 & Li-ion & 1.2 & 0.5 & 2.4 & 0.7 & 2.0 & 0.6 & 0.7 & 0.2 \\
C4 & Flywheel & 1.2 & 0.4 & 2.4 & 0.6 & 2.0 & 0.6 & 0.7 & 0.2 \\
C5 & CAES 2 modes & 2.3 & 13.4 & 4.2 & 23.8 & 3.7 & 22.1 & 1.3 & 7.3 \\
& 7-min waiting period, Na-S & 0.6 & 0.1 & 0.9 & 0.1 & 1.2 & 0.1 & 0.4 & 0.05 \\
C6 & Flow battery & 1.2 & 0.5 & 2.4 & 0.7 & 2.0 & 0.6 & 0.7 & 0.2 \\
C7 & PH multiple modes & 1.2 & 0.4 & 2.4 & 0.6 & 2.0 & 0.6 & 0.7 & 0.2 \\
& 4-min waiting period, Na-S & 0.5 & 0.1 & 0.9 & 0.2 & 0.9 & 0.1 & 0.3 & 0.1 \\
C8 & PH 2 modes & 2.3 & 13.5 & 4.2 & 23.9 & 3.7 & 22.2 & 1.3 & 7.4 \\
& 4-min waiting period, Na-S & 0.5 & 0.03 & 0.7 & 0.05 & 0.9 & 0.05 & 0.4 & 0.03 \\
C9 & DR & 4.3 & - & 8.5 & - & 7.19 & - & 2.37 & - \\
\hline
\end{tabular}

Figure ES.2 show both the additional intra-hour and the total intra-hour balancing requirements to integrate about $14.4 \mathrm{GW}$ of additional wind to a total installed wind power capacity of about $24 \mathrm{GW}$ in the WECC. The power requirements for the additional intra-hour balancing services to add about 14.4 GW of wind capacity in the WECC are about $1.53 \mathrm{GW}$. If provided by energy storage, the energy capacity requirements are $0.58 \mathrm{GWh}$ or a 23 minute storage (23 minutes on average in WECC, ranging between 20 and 27 minutes across the four regions).

For the total intra-hour balancing services, the power requirements are estimated to be $6.3 \mathrm{GW}$ by 2020 with an energy capacity of 2.0 GWh or a 19 minute storage (19 minutes on average in WECC, ranging from 17 to 23 minutes across the four regions). 


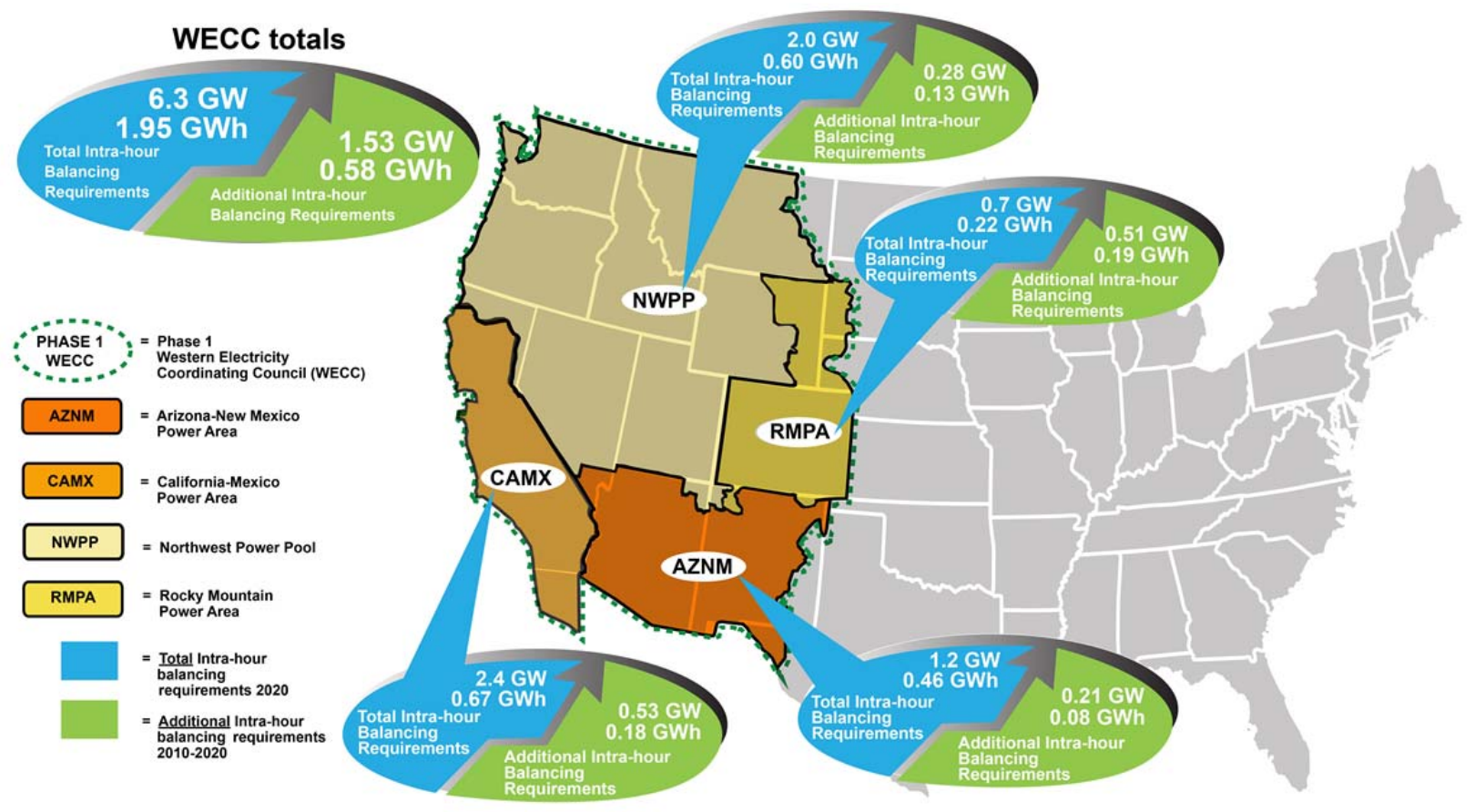

Figure ES.2. Size Requirements for Storage Technologies to Meet Additional and Total Intra-Hour Balancing Requirements for a 2020 Grid with 20\% RPS

\section{Outcome 3: Competitiveness of Storage Technologies: Na-S Batteries, Flywheels Pumped Storage, and Demand Response compete today, Li-Ion and Redox Flow are likely to be competitive in $\mathbf{2 0 2 0}$}

Various technologies compete for the growing balancing market opportunities, not only energy storage, but also demand response. The base case technology is a gas-fueled CT, which may be attractive particularly under low cost gas projections for the next decades. The LCC analysis for intra-hour service indicated that Na-S, flywheel storage technologies, pumped hydro storage with multiple mode changes, and DR are under current cost estimates are already cost-competitive (lowest LCC). Li-ion and Redox flow will follow under cost reduction assumption for the 2020 timeframe. These findings are consistent among the four sub-regions and are applicable across other regions. Figure ES.3 presents the results of the LCC analysis and the effects of capital, O\&M (operations and maintenance), emissions, and fuel costs on the total LCC for each case, as applied in the NWPP. 


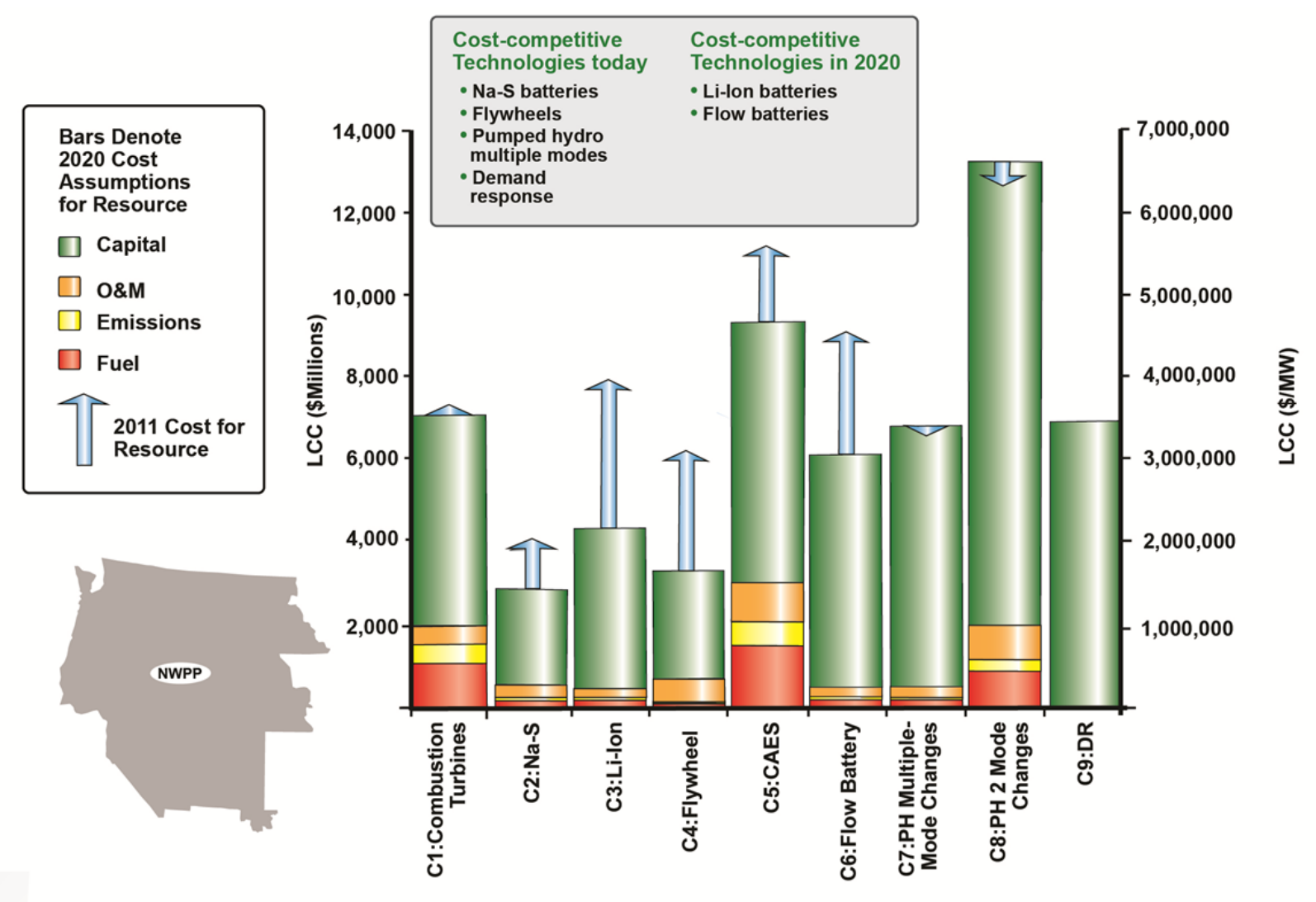

Note: Cost ranges include key uncertainties in the 2011 and 2020 cost assumptions

Figure ES.3. Scenario LCC Estimates ${ }^{1}$

The detailed LCC modeling effort was used to assess the cost competitiveness of different technologies to address the future, intra-hour balancing requirements. The cost analysis considered the costs associated with initial and recurrent capital costs, fixed and variable O\&M costs, emissions costs, and fuel costs. Annualized costs incurred over a 50-year time horizon were collapsed into a single present value cost for each scenario using a nominal discount rate of $8 \%$, across all cases. There is a significant degree of capital cost uncertainty associated with the energy storage technologies, especially for cases evaluated farther into the future. A constant discount rate across all technologies may tend to underestimate the risk associated with some technologies, particularly those technologies at an earlier technology readiness level (TRL) stage. The future cost ranges were determined on an individual basis, based on conversations with vendors, assessment of novel materials that would enable cost cutting, and the risk of these assumptions not coming to fruition.

LCC results are strictly applicable for intra-hour balancing services with an average cycle time of about 20-30 minutes. As the application requires longer cycle times with higher energy capacity, capital costs and production cost of conventional generators will be different, all affecting the LCC results and the relative cost competitiveness.

\footnotetext{
${ }^{1}$ Note that the costs of implementing DR are assumed to be $\$ 50.70$ per kW per year as estimated in EPRI (2009). This value includes all costs required to install, operate, and maintain DR and DR-enabling equipment.
} 


\section{Outcome 4: Energy Storage Devices are not Expected to Achieve Cost Recovery when Deployed for Arbitrage Services}

Energy arbitrage alone is insufficient to provide sufficient revenues to make new energy storage installations economically viable even in congested paths such as transfer into Southern California and interchange at the California-Oregon border. Although this result was based on the production cost modeling that estimates cost differential between peak and off-peak, not market price differentials, which tends to be higher than the cost differentials, the frequency and duration of transmission congestions were simply not sufficient to make energy storage technologies a viable business proposition as an energy product. It will require other value streams such as a capacity payments that are valued more highly that the average energy value to augment the revenue stream for storage capacities. Potentially, with sufficiently large duration of energy storage beyond 4 hours, capital deferment in the bulkpower system and at the substation could provide additional revenues such that longer duration storage could be economically viable.

The revenue potential of arbitrage was determined by identifying constraints in the system and determining the locational marginal price (LMP) differential for hours throughout the year along those congested paths. Simulations were then carried out for different energy storage sizes for the NWPP and CAMX. The other regions (AZNM and RMPA) did not indicate significant congestion paths that would suggest to explore storage as a mitigation strategy. The results of the WECC simulation are presented in Table ES.3. Arbitrage revenue estimates in the WECC grow from $\$ 4.3$ million annually at $240 \mathrm{MW} / 10$ hour storage to $\$ 99.1$ million for a 9,712 MW/10 hour storage capacity.

Annual costs are also presented in Table ES.4, demonstrating that arbitrage revenue expectations fall short of the revenue requirements necessary for cost recovery. This general finding applies to each scenario presented in this report. Thus, it would take either a significant increase in the peak- to off-peak LMP differential for extended periods of time or reductions in energy storage capital costs for energy arbitrage as designed in this analysis to break even. Under the current projections for the WECC, even the $243 \mathrm{MW}$ scenario fails to yield profits when applied to the most profitable hours of the year. This result supports the conclusion that at a 30\% reserve margin, the WECC region is not sufficiently congested for energy storage to become cost-effective when used to provide only arbitrage services. 
Table ES.4. Annualized Revenue and Capital Costs for Na-S Batteries, Li-Ion Batteries, and Pumped Hydro

\begin{tabular}{cccccc}
\hline \multicolumn{2}{c}{ Storage Size } & \multirow{2}{*}{$\begin{array}{c}\text { Annual } \\
\text { Revenue }\end{array}$} & \multicolumn{3}{c}{ Annualized Costs (in \$ mill.) } \\
\cline { 5 - 6 } (in \$mill.)
\end{tabular}

While the findings of this analysis indicate that profits from energy arbitrage are insufficient to achieve capital cost recovery, it is important to note that there are several other services that could be supplied by energy storage technologies that were excluded from this assessment. These services include load following, transmission and distribution upgrade deferral, grid stability management, power quality enhancements, electricity service reliability, and additional reserve capacity. The valuation of these services and grid benefits, particularly, when provided simultaneously is immature or highly site-specific and, thus, beyond the scope of this assessment. Additional research is therefore necessary to examine the full revenue potential of energy storage used in multiple applications.

This study treats pumped hydro as an asset whose performance attributes are consistent with presently technology presently installed in the U.S. New pumped hydro technology (not yet deployed in the U.S.) reportedly has faster response than available from the existing U.S. facilities. Consideration of the role of fast response hydro and pumped hydro in meeting grid balancing requirements should be addressed in future studies, as cost performance information becomes available.

\section{Outcome 5: Hybrid System Offer No Technical Performance Advantages, Therefore Will Have to Compete on Cost Alone}

The analysis of the optimal hybrid energy storage system offered results that were solely driven by cost. The minute-by-minute simulation did not provide sufficient resolution in the time domain to expose ramping limits of all of the tested energy storage technologies. Thus, differences in the ramp rates across all studied technologies were not a differentiator in the optimal hybridization of storage systems. The results clearly indicated a "winner-take-all” solution. As a consequence all of the attempts to optimally pair two individual technologies resolved to one, and only one, of the two technologies. There was only one particular case, where the cost-optimal solution indicated a bundling of two technologies.

For the lithium-ion (Li-ion) and DR case under the 2011 price scenario, the cost-optimal bundling suggested $60 \%$ of DR and $40 \%$ of Li-ion because of a non-constant availability of the demand response resource. The DR resource was assumed to be smart charging strategies of EVs (i.e., variable charging 
about an operating point of charging). The availability of the resource is high after the morning commute when the vehicles are assumed to be recharged at work, likewise, when the vehicles come home and being recharged at home for the next day. There times when the EV fleet is almost fully charged (very early in the morning 3-5 a.m.), thus the DR resource is very low. At that time the Li-ion stationary batteries must be used to off-set the lack of DR resource. The optimum tradeoff between DR and stationary Li-ion batteries for the 2011 cost estimates was 60/40 share of DR and battery. As the cost for the Li-ion stationary battery drops relative to the DR (as for the 2020 cost estimate) the optimal pairing suggested a 0/100 share between DR and the battery.

The key message of the hybrid storage analysis suggests that hybridizing storage technologies will only be meaningful if there are a wide spectrum of cycles expected with sharp transients with sub-oneminute time resolution, which this analysis did not expose. Alternatively, energy storage may function in concert with DR or other generators (as a virtual hybrid system) to compensate for their lack of availability or ramping capability. 


\section{Acknowledgments}

We are particularly thankful to Dr. Imre Gyuk, manager of the Energy Storage System Program of the U.S. Department of Energy, Office of Electricity Delivery and Energy Reliability for providing the funding for this project.

We would like to thank the following PNNL staff: Dr. Daiwon Choi for several useful discussions on Li-ion battery materials development. Dr. Gary Yang ${ }^{1}$, and especially Dr. Soowhan Kim and Dr. Liyu Li ${ }^{2}$ for providing significant information on battery performance trends.

We would also like to thank Dr. Lawrence Thaller, Consultant for helping with developing a cost model for redox flow batteries.

\footnotetext{
${ }^{1}$ Dr. Yang left PNNL during the final edits of this report.

${ }^{2}$ Dr. Li left PNNL during the final edits of this report.
} 



\section{Acronyms and Abbreviations}

\begin{tabular}{|c|c|}
\hline ACE & area control error \\
\hline AEO & Annual Energy Outlook \\
\hline ANL & Argonne National Laboratory \\
\hline AZNM & Arizona-New Mexico-Southern Nevada (sub-region of the WECC) \\
\hline BA & balancing authority \\
\hline BASF & Badische Anilin- und Soda-Fabrik, Ludwigshafen, Germany \\
\hline $\mathrm{BC}$ & British Columbia \\
\hline BOP & balance of plant \\
\hline Btu & British Thermal Unit \\
\hline $\mathrm{BPA}$ & Bonneville Power Administration \\
\hline CAISO & California Independent System Operator \\
\hline CAMX & $\begin{array}{l}\text { California-Mexico. Only a small region of the Baja peninsula is included (sub- } \\
\text { region of the WECC) }\end{array}$ \\
\hline $\mathrm{CC}$ & combined cycle \\
\hline CCGT & combined cycle gas turbine \\
\hline CT & combustion turbine \\
\hline CAES & compressed air energy storage \\
\hline DOD & depth of discharge \\
\hline $\mathrm{DR}$ & demand response \\
\hline EIA & Energy Information Administration \\
\hline $\mathrm{E} / \mathrm{P}$ & energy/rated power \\
\hline EPA & Environmental Protection Agency \\
\hline EPRI & Electric Power Research Institute \\
\hline ERCOT & Electric Reliability Council of Texas \\
\hline ESPC & Energy Storage and Power Corporation \\
\hline ESS & Energy Storage Systems \\
\hline $\mathrm{EV}$ & Electric Vehicle \\
\hline GW & gigawatt \\
\hline GWh & gigawatt-hours \\
\hline ID & Idaho \\
\hline ICAP & installed capacity (NYISO capacity market) \\
\hline ISO & independent system operator \\
\hline KEMA & Keuring Electrotechnisch Materieel Arnhem \\
\hline $\mathrm{kW}$ & kilowatt \\
\hline kWh & kilowatt-hour \\
\hline LCC & life-cycle cost \\
\hline
\end{tabular}


LHV

Li-ion

LMP

LTC

MRL

MW

MWh

MISO

$\mathrm{Na}-\mathrm{S}$

$\mathrm{NO}_{\mathrm{x}}$

NREL

NERC

NWPP

NYISO

O\&M

OR

$\mathrm{P} / \mathrm{E}$

PCS

$\mathrm{PH}$

PHES

PHEV

PNNL

PROMOD

redox

RPS

RMPA

RD\&D

SA

SCE

$\mathrm{SO}_{2}$

TRL

TEPPC

TSI

USABC

V2G

$\mathrm{V}_{2} \mathrm{O}_{5}$

WECC lower heating value

lithium-ion

locational marginal piece

Lithium Technology Corp

manufacturing readiness level

megawatt

megawatt-hour

Midwest Independent Transmission System Operator

sodium sulfur

nitrogen oxides

National Renewable Energy Laboratory

North American Electric Reliability Corporation

Northwest Power Pool (sub-region of WECC)

New York Independent System Operator

operations and maintenance

Oregon

power to energy

power conversion system

pumped hydroelectric

pumped hydro energy storage

plug-in hybrid electric vehicles

Pacific Northwest National Laboratory

production cost modeling software by Ventyx

reduction-oxidation

renewable portfolio standards

Rocky Mountain Power Area (sub-region of the WECC)

research, development, and demonstration

Sensitivity Analysis

Southern California Edison

sulfur dioxide

technology readiness level

Transmission Expansion Planning and Policy Committee

Tribology Systems Inc.

US Advanced Battery Consortium

vehicle-to-grid

vanadium oxide

Western Electricity Coordinating Council 


\section{Contents}

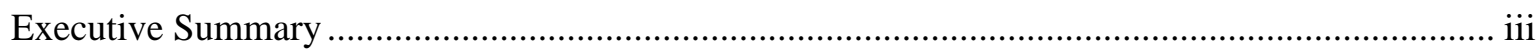

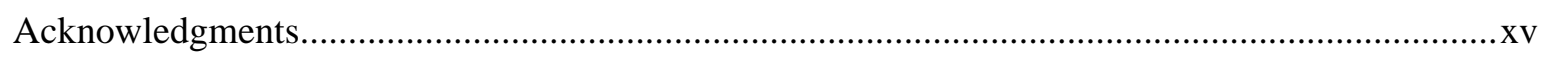

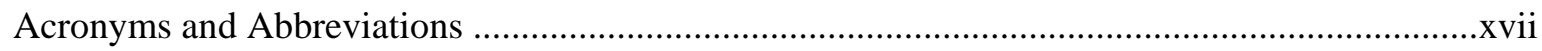

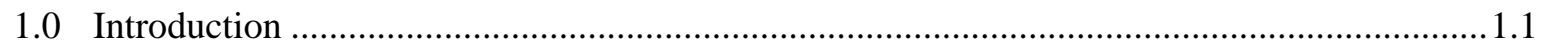

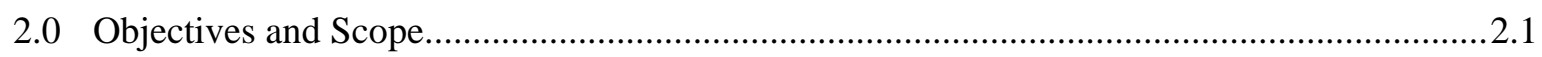

3.0 How is this Assessment Different from Other Studies? ........................................................

4.0 Technology Choices for Balancing Services .................................................................... 4.1

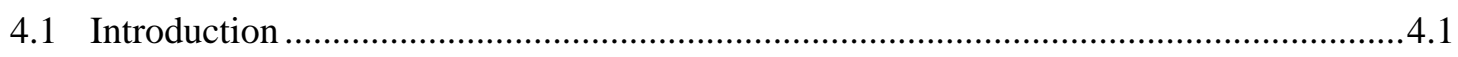

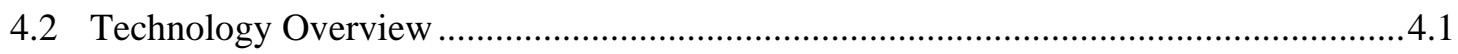

4.2.1 Combustion Turbine...................................................................................... 4.1

4.2.2 Combined Cycle Plant................................................................................ 4.1

4.2.3 Compressed Air Energy Storage .....................................................................4

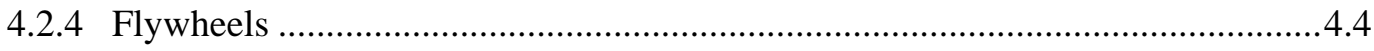

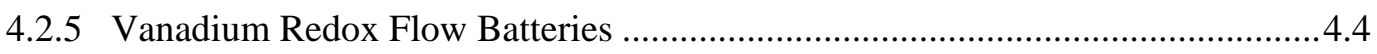

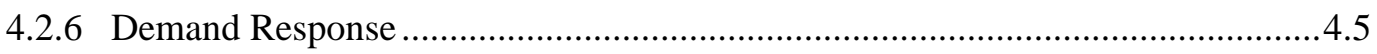

4.3 Technology Cost and Performance Characteristics..................................................... 4.5

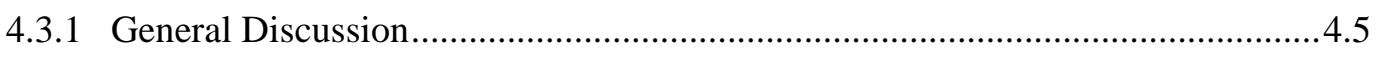

4.3.2 Summary of Capital, O\&M Costs, and Efficiency for Batteries and PH .............4.7

4.4 Definition of Technology Options ............................................................................. 4.9

5.0 Methodology for Estimating Balancing Requirements …............................................... 5.1

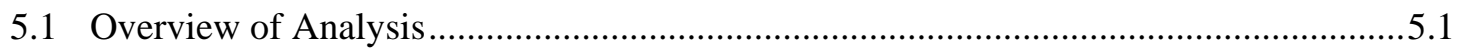

5.2 Approach and Data Used to Determine Balancing Requirements ..................................5.1

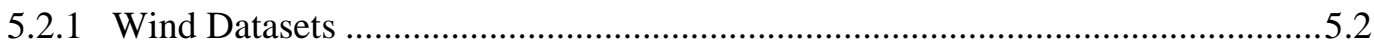

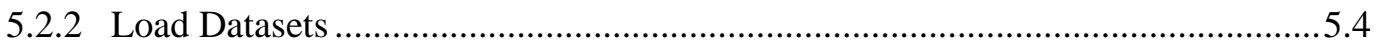

5.2.3 Balancing Service Requirement .....................................................................5.5

5.2.4 Consolidation of Balancing Areas................................................................... 5.6

5.2.5 Resulting Total and Additional Balancing Signals .............................................5.7

5.2.6 Spectral Analysis and Extraction of Intra-Hour Balancing Signal........................5.8

5.3 Sizing Storage to Meet Balancing Requirements ......................................................10

5.3.1 Sizing Hybrid Technology Options for Balancing Services ..............................5.10

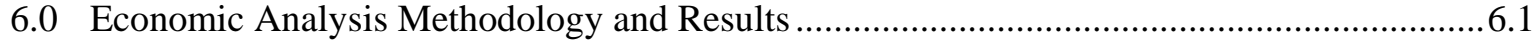

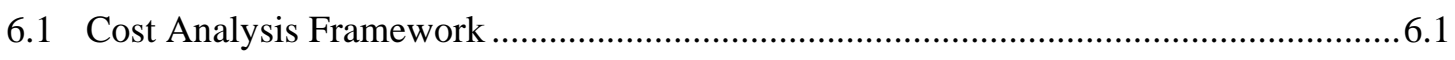

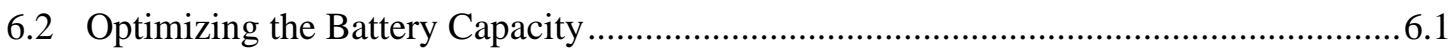

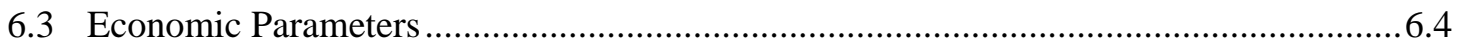

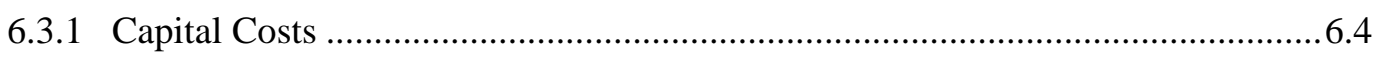

6.3.2 Operations and Maintenance Costs ….................................................................. 6.5 


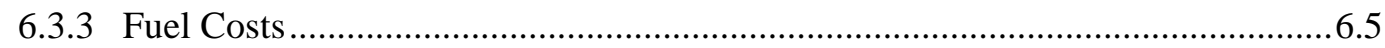

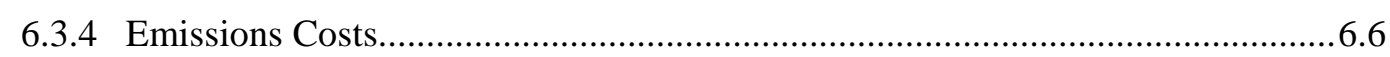

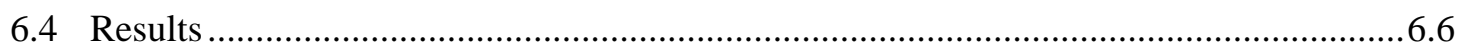

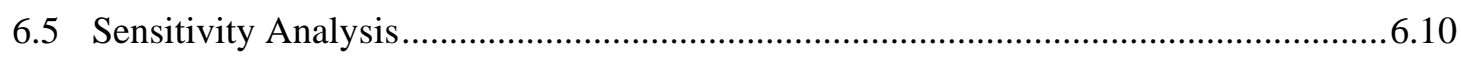

7.0 Arbitrage Opportunities for Energy Storage................................................................... 7.1

7.1 Arbitrage Analysis Framework ….............................................................................

7.1.1 General discussion..................................................................................... 7.3

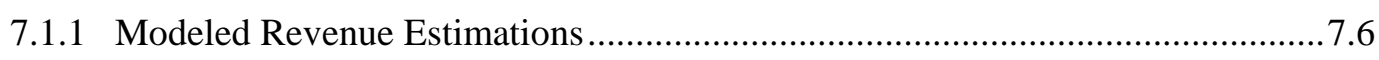

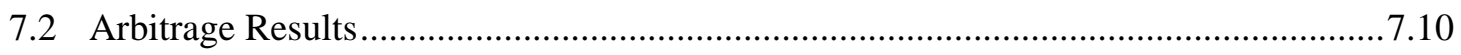

7.3 Final Observations on Arbitrage Results................................................................16

8.0 Results: Balancing Requirements ................................................................................... 8.1

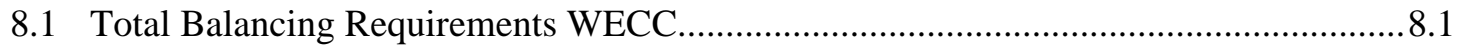

8.2 Additional Balancing Requirements for WECC ........................................................ 8.3

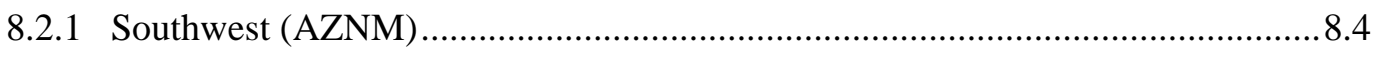

8.2.2 Energy and Power Requirements ......................................................................8.6

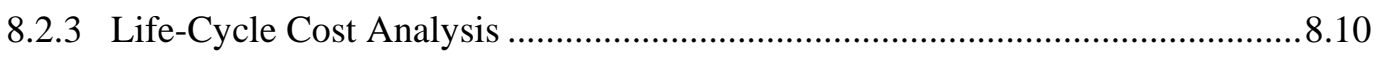

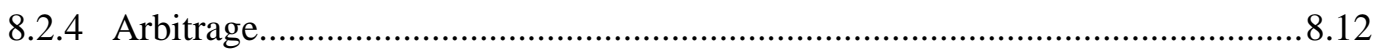

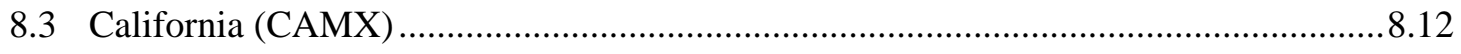

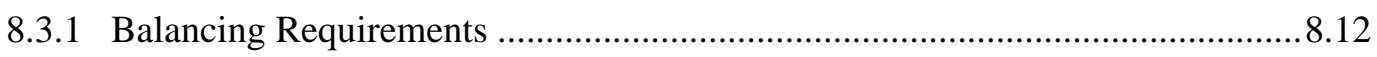

8.3.2 Life-Cycle Cost Analysis .............................................................................. 8.14

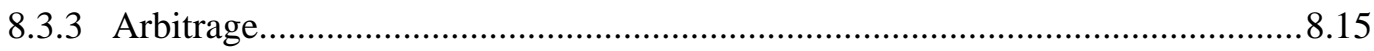

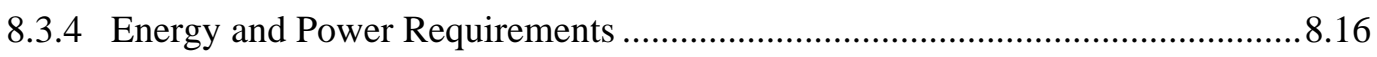

8.4 Northwest Power Pool (NWPP) ............................................................................. 8.19

8.4.1 Balancing Requirements ..............................................................................19

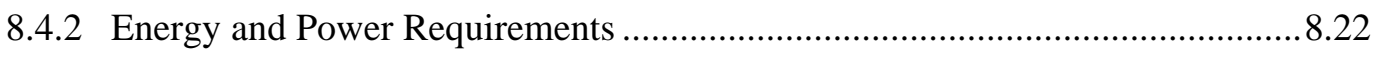

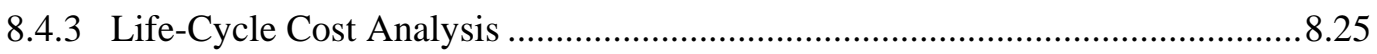

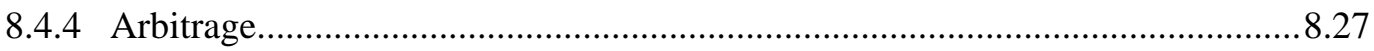

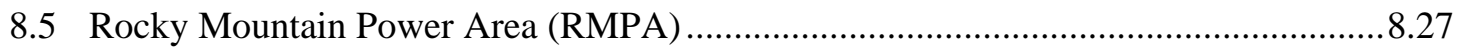

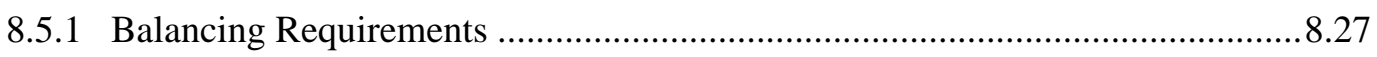

8.5.2 Energy and Power Requirements ................................................................. 8.30

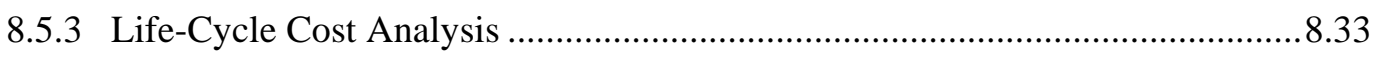

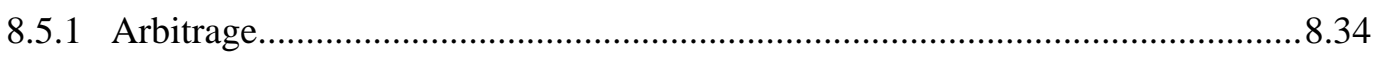

8.5.2 Sensitivity Analysis................................................................................. 8.34

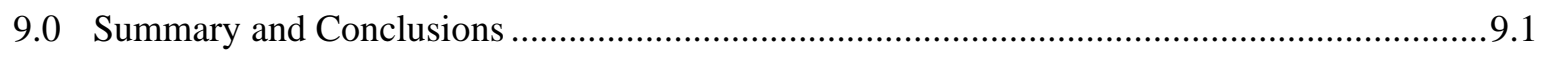

9.12020 Balancing Requirements ............................................................................. 9.1

9.2 Market Size for Energy Storage for Balancing Services................................................2

9.3 Life-Cycle-Cost Analysis of Various Technology Options to Meet Future Balancing

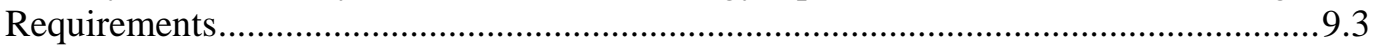

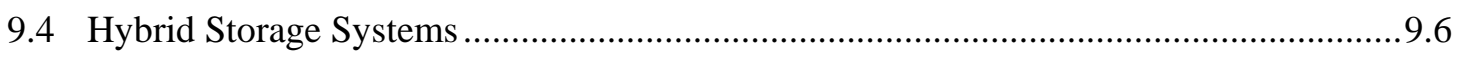




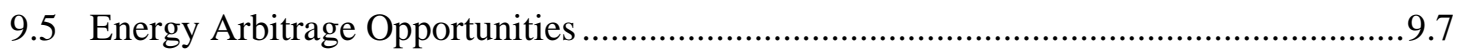

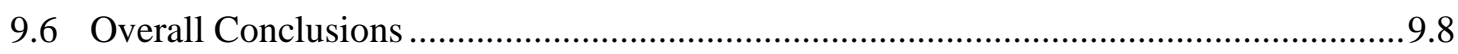

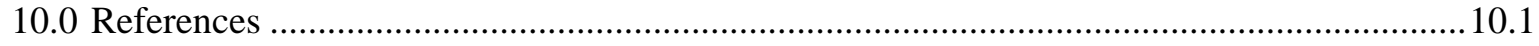

Appendix A - Detailed Technology Discussion ...................................................................... 1

Appendix B - Specific Operational Strategies to Meet Balancing Requirements ........................ B.1

Appendix C - Additional Balancing Requirements and Report Differentiators............................. C.1

Appendix D - Detailed Desciption of Energy Storage Analysis Landscape ................................ D.1 


\section{Figures}

ES.1 Regional Resolution of the National Assessment ...................................................... iv

ES.2 Size Requirements for Storage Technologies to Meet Additional and Total Intra-Hour Balancing Requirements for a 2020 Grid with 20\% RPS .......................................... ix

ES.3 Scenario LCC Estimates …....................................................................................

1.1. Energy storage and market assessment, conducted by Sandia National Laboratories.............1.2

1.1. Energy storage and market assessment, conducted by Sandia National Laboratories (Eyer and Corey, 2010).

2.1. Spatial Definition of Regions based on NERC-Regionalization (DOE/EIA 2011) ................2.3

4.1. Balancing Signal and Load Resource Curve for PHEV with Home and Work Charging ....... 4.6

5.1. Wind Projection 2011-2020 for NERC regions in WECC ..................................................5.2

5.2. Distribution of Wind Capacity by States ...............................................................................

5.3. Location of Existing and Arbitrarily Sited Future Wind Plants in WECC .............................5.4

5.4. Illustration of Intra-Hour Balancing Signal ......................................................................

5.5. An Example of Total Balancing Requirements for the Month of August 2020 ....................5.7

5.6. An Example of Total Balancing Requirements for One Typical Day in August 2020............5.8

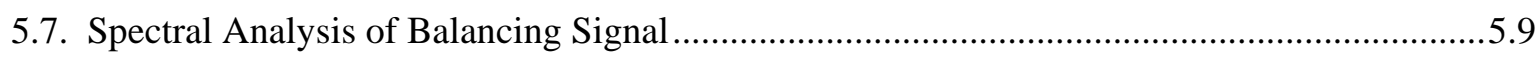

5.8. Components of Decomposed Balancing Signal .....................................................................

5.9. Division of Balancing Signal for Two Storage Technologies ...........................................5.11

5.10. Storage Sizes in Terms of Energy Requirement (kWh) for the Two Storage Technologies5.11

5.11. Storage Sizes in Terms of Power Requirement (kW) for the Two Storage Technologies...5.12

5.12.Storage Sizes in Terms of Maximum Ramp Rate Requirement (MW/min) for the Two

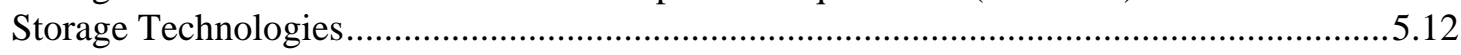

5.13.Total 50-Year LCCs for Li-ion +DR Technology Shares for 2020 Cost Assumptions. "Winner takes all” situation present in most cases studied..................................................5.13

5.14.Total 50-Year LCCs for Li-ion +DR Technology Shares for 2011 Cost Assumptions. Optimal combination (technology share 6) only present in two cases under 2011 cost assumptions.

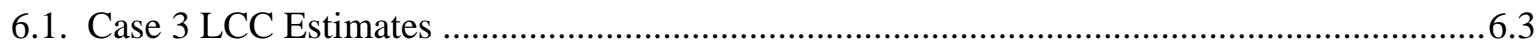

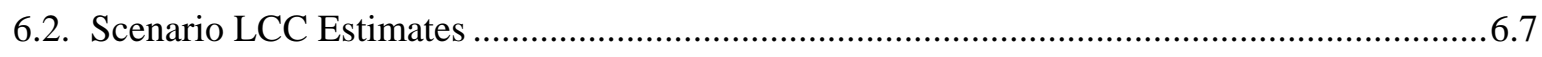

6.3. LCC Estimates Adjusted due to Capital Cost Variability...................................................6.10

7.1. (a) LMP Differential per Hour of Operation (\$/MWh) and (b) Marginal Revenue per Hour of Operation (\$/MWh)

7.2. Dependency of Capital Cost for Storage Component $C_{\text {sto }}$ in $(\$ / \mathrm{kWh})$ on Peak-Off Peak Ratio and Efficiency. Assumed: $p_{o}=\$ 40 / \mathrm{MWh}, D=260$ days, $\alpha=0.12$.

7.3. Dependency of Capital Cost for Storage Component $C_{\text {sto }}$ in $(\$ / \mathrm{kWh})$ on Peak-Off Peak Ratio and Efficiency. Assumed: $p_{o}=\$ 40 / \mathrm{MWh}, D=260$ days, $\alpha=0.12$, and capacity value of $\$ 150$ per kW per year. 
7.4. Dependency of Capital Cost for Storage Component $C_{\text {sto }}$ in $(\$ / \mathrm{kWh})$ on Peak-Off Peak Ratio and Capacity Value. Assumed: $p_{o}=\$ 40 / \mathrm{MWh}, D=260$ days, $\alpha=0.12$, and efficiency of $\eta=0.85$..

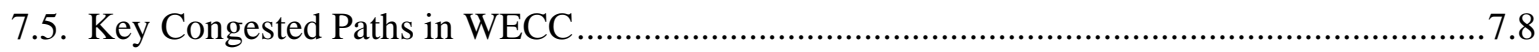

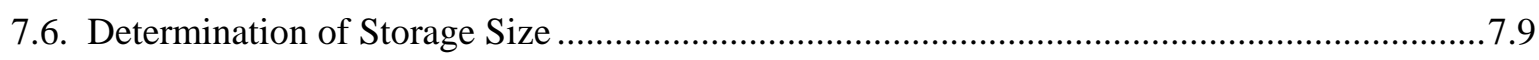

7.7. Arbitrage Revenues in NWPP, CAMX, and WECC per Year ….......................................7.10

7.8. Total and Marginal Arbitrage Revenues in WECC per Year ..............................................7.11

7.9. Arbitrage Revenues plus Capacity Values in NWPP, CAMX, and WECC per Year............7.12

8.1. One Month Total Balancing Signal of August 2020 for AZNM ...........................................8.4

8.2. One Typical Day Total Balancing Signal of August 2020 for AZNM .................................8.5

8.3. One Month Balancing Requirements Caused by Load and Wind Respectively for AZNM ...8.5

8.4. One Typical Day Balancing Requirements Caused by Load and Wind Respectively for AZNM.

8.5. Power Requirements for all the Technologies to Meet Balancing Signal for AZNM ............8.7

8.6. Energy Requirements for Storage Technologies to Meet Balancing Signal for AZNM..........8.8

8.7. Power Requirements for all the Technologies to Meet Balancing Signal due to 2011-2020 Additional Wind and Load Signal for AZNM.....

8.8. Energy Requirements for Storage Technologies to Meet Balancing Signal due to 2011-2020

Additional Wind and Load Signal for AZNM .....................................................................

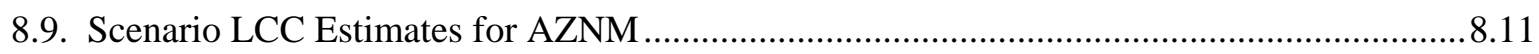

8.10. One Month Total Balancing Signal of August 2020 for CAMX ......................................8.12

8.11. One Typical Day Total Balancing Signal of August 2020 for CAMX ...............................8.13

8.12. One Month Balancing Requirements Caused by Load and Wind, Respectively for CAMX8.13

8.13. One Typical Day Balancing Requirements Caused by Load and Wind, Respectively for CAMX

8.14. Scenario LCC Estimates (California-Mexico Power Area) ...............................................15

8.15. Power Requirements for all the Technologies to Meet Balancing Signal for CAMX .........8.17

8.16. Energy Requirements for Storage Technologies to Meet Balancing Signal for CAMX .....8.18

8.17.Power Requirements for all the Technologies to Meet Balancing Signal due to 2011-2020 Additional Wind and Load for CAMX.

8.18.Energy Requirements for Storage Technologies to Meet Balancing Signal due to 2011-2020

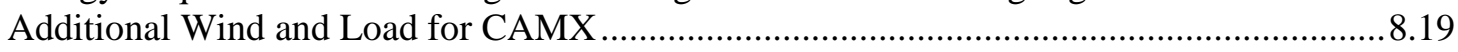

8.19. One Month Total Balancing Signal of August 2020 for NWPP ......................................8.20

8.20. One Typical Day Total Balancing Signal of August 2020 for NWPP..............................8.20

8.21. One Month Balancing Requirements Caused by Load and Wind, Respectively for NWPP8.21

8.22. One Typical Day Balancing Requirements Caused by Load and Wind, Respectively for NWPP

8.23. Power Requirements for all the Technologies to Meet Balancing Signal for NWPP ..........8.23

8.24. Energy Requirements for Storage Technologies to Meet Balancing Signal........................8.24

8.25.Power Requirements for all the Technologies to Meet Balancing Signal due to 2011-2020 Additional Wind and Load for NWPP. 
8.26.Energy Requirements for Storage Technologies to Meet Balancing Signal due to 2011-2020

Additional Wind and Load for NWPP

8.27.Scenario LCC Estimates (NWPP)

8.28. One Month Total Balancing Signal of August 2020 for RMPP 8.28

8.29. One Typical Day Total Balancing Signal of August 2020 for RMPP

8.30. One Month Balancing Requirements Caused by Load and Wind Respectively for RMPP.8.29

8.31.One Typical Day Balancing Requirements Caused by Load and Wind Respectively for RMPP.

8.32. Power Requirements for all the Technologies to Meet Balancing Signal for RMPP

8.33. Energy Requirements for Storage Technologies to Meet Balancing Signal for RMPP

8.34.Power Requirements for all the Technologies to Meet Balancing Signal due to 2011-2020 Additional Wind and Load for RMPP

8.35.Energy Requirements for Storage Technologies to Meet Balancing Signal due to 2011-2020 Additional Wind and Load for RMPP 8.33

8.36.Scenario LCC Estimates for RMPP 8.34

8.37. Impacts of Wind Forecast Error on Incremental Power Requirements 8.35

8.38. Impacts of Wind Forecast Error on Decremental Power Requirements 8.35

9.1. Size Requirements for Storage Technologies to Meet the Total and Additional Intra-Hour Balancing Services for a 2020 Grid with 20\% RPS.............................................................. 9.3

9.2. Scenario LCC Estimates .9 .5

9.3. Total 50-Year LCCs for Li-Ion +DR Technology Shares for 2011 Cost Assumptions. Optimal combination (technology share 6) only present in two cases under 2011 cost assumptions. 


\section{Tables}

ES.1 Additional and Total Intra-Hour Balancing Requirements for WECC Sub-Regions in 2020 for $20 \%$ RPS. vi

ES.2 Power and Energy Requirements by Technology Case to Meet Additional Intra-Hour Balancing Requirements........................................................ vii

ES.3 Power and Energy Requirements by Technology Case to Meet Total Intra-Hour Balancing Requirements. viii

ES.4 Annualized Revenue and Capital Costs for Na-S, Li-Ion Batteries, and Pumped Hydro...xii

3.1. Characterization of Major Storage Studies 3.2

4.2. Summary of Capital and O\&M Costs for Technologies Analyzed. Note values are representative for 2011 technologies. 2020 values are in parentheses.

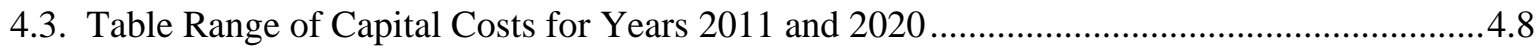

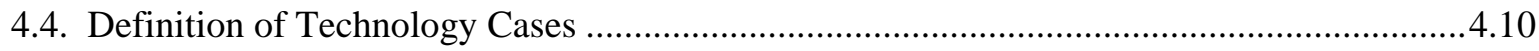

5.1. Information About NREL Wind Integration Datasets ....

5.2. Statistics of Hour-Ahead Wind Forecast Error (the percentage values are based on installed wind capacity)

5.3. Statistics of Hour-Ahead Load Forecast Error ........................................................................5.5

5.4. Frequency Limits of Components of the Balancing Signal .....................................................5.9

6.1. Utility Description Data and General Economic Parameters................................................6.2

6.2. Relationship between DOD, Battery Capacity, and Life Cycle.............................................. 6.2

6.3. Cost Minimizing DOD, Battery Capacity, and Economic Life by Case................................6.4

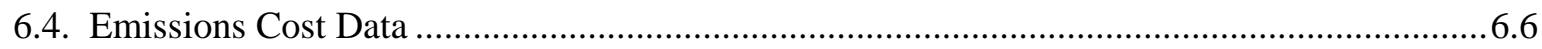

6.5. Economic Analysis Results (in Million 2011 Dollars) …...................................................6.7

6.6. Economic Analysis Results - Base Case vs. New Load and Wind Capacity Case (in million 2011 dollars)

6.7. Capital Cost Range for Various Technologies in Year 2020 .............................................6.9

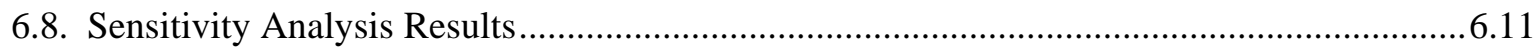

7.1. Existing and Additional Installed Capacity (MW) for AZNM, CAMX, NWPP, and RMPA

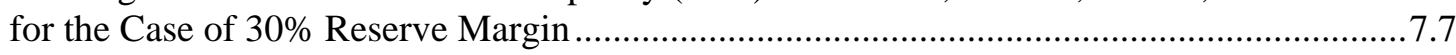

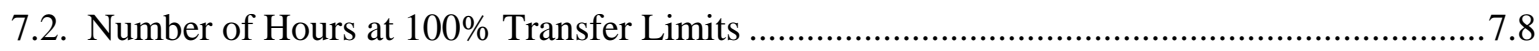

7.3. Annual Arbitrage Revenues in NWPP, CAMX, and WECC by Energy Storage Capacity

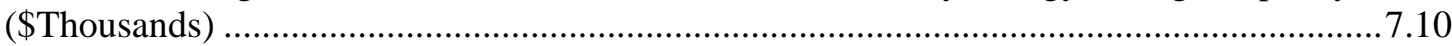

7.4. Total and Marginal Arbitrage Revenues in WECC (\$Thousands per MW per year) ............7.11

7.5. Annual Arbitrage and Capacity Reserve Revenues in NWPP, CAMX, and WECC by Energy Storage Capacity .

7.6. Annualized Capital Costs for Na-S Batteries, Li-Ion Batteries, and Pumped Hydro .............7.14

7.7. Annualized Profits for Na-S Batteries, Li-Ion Batteries, and Pumped Hydro (Capacity Value Excluded)

7.8. Annualized Profits for Na-S Batteries, Li-Ion Batteries, and Pumped Hydro in WECC with Capacity Value and Arbitrage Values. 
8.1. Total Balancing Requirements for every NERC Region in WECC.

8.2. Intra-hour Balancing Requirements for NERC Sub-Regions in WECC.

8.3. Total Balancing Requirements Caused by Wind Uncertainty

8.4. Total Balancing Requirements Caused by Load Uncertainty ...

8.5. Balancing Requirements (Intra-hour) for NERC Sub-Regions in WECC caused by Wind Variability Only (without considering load variability)

8.6. Balancing Requirements (Intra-hour) for NERC Sub-Regions in WECC caused by Load Variability Only (without considering wind variability) ....

8.7. Additional Balancing Requirements for every NERC Region in WECC.....

8.8. Power and Energy Requirements for Each Scenario for AZNM. Note: The energy capacity (GWh) for the batteries is nominated at a DOD of $100 \%$.

8.9. Power and Energy Requirements for Each Scenario due to 2011-2020 Additional Wind and Load Signal for AZNM. Note: The energy capacity (GWh) for the batteries is noted at a DOD of $100 \%$.

8.10. Economic Analysis Results - AZNM (2020 Prices).....

8.11. Economic Analysis Results - California-Mexico Power Area (2020 Prices).

8.12. Annualized Capital Costs for Na-S Batteries, Li-Ion Batteries, and Pumped Hydro (CAMX).

8.13.Power and Energy Requirements for Each Scenario for CAMX. Note: The energy capacity (GWh) for the batteries is nominated at a DOD of $100 \%$.

8.14.Power and Energy Requirements for Each Scenario due to 2011-2020 Additional Wind and Load for CAMX. Note: The energy capacity (GWh) for the batteries is nominated at a DOD of $100 \%$.

8.15.Power and Energy Requirements for Each Scenario for NWPP. Note: The energy capacity (GWh) for the batteries is nominated at a DOD of $100 \%$.

8.16.Power and Energy Requirements for Each Scenario due to 2011-2020 Additional Wind and Load for NWPP. Note: The energy capacity (GWh) for the batteries is nominated at a DOD of $100 \%$.

8.17. Economic Analysis Results for NWPP (2020 Prices)...

8.18. Annualized Capital Costs for Na-S Batteries, Li-Ion Batteries, and Pumped Hydro (NWPP)8.27

8.19.Power and Energy Requirements for Each Scenario for RMPP. Note: The energy capacity (GWh) for the batteries is nominated at a DOD of $100 \%$.

8.20.Power and Energy Requirements for Each Scenario due to 2011-2020 Additional Wind and Load for RMPP. Note: The energy capacity (GWh) for the batteries is nominated at a DOD of $100 \%$.

8.21. Economic Analysis Results - RMPA (2020 Prices).....

8.22. Total Balancing Requirements of NWPP Based on 20 Simulations ...8.36

9.1. Total and Intra-Hour Balancing Requirements for every NERC Region in WECC in 2020...9.1

9.2. Additional Intra-Hour and Total Intra-Hour Balancing Requirements for WECC SubRegions

9.3. Definition of Technology Cases

9.4. Annualized Revenue and Capital Costs for Na-S Batteries, Li-Ion Batteries, and Pumped Hydro 
xxvii 



\subsection{Introduction}

Energy storage systems have the potential to improve the operating capabilities of the electricity grid. Their ability to store energy and deliver power can increase the flexibility of grid operations while providing the reliability and robustness that will be necessary in the grid of the future - one that will be able to provide for projected increases in demand and the integration of clean energy sources while being economically viable and environmentally sustainable.

Driven by the current RPS established in 31 of the nation's states, the total contribution of renewable resources to the electricity generation portfolio in the United States is expected to grow significantly in the 2015 to 2025 timeframe. The President's clean energy goals of 80\% renewable energy by 2050 will require further accelerated deployment of renewable energy resources. The projected increase of these sources will necessitate the deployment of technologies that can address renewable variability in an environmentally sustainable fashion. Energy storage embraces a suite of technologies that have the potential for deployment to assist the increasing penetration of renewable resources. While other technologies, such as gas turbine and transmission upgrades can provide operational flexibility, energy storage has the unique ability to both improve asset use and meet the flexibility needs with one technology. Most energy storage systems have superior ramping characteristics compared to rotary turbomachinery such as combustion or steam turbines, and provide more effective area control error (ACE) compensation than do turbine-based generators (FERC NOPR 2011; Makarov 2008b).

The Energy Storage Systems (ESS) Program within the U.S. Department of Energy, Office of Electricity Delivery \& Energy Reliability (DOE-OE) is taking a lead role in the research, development, and demonstration (RD\&D) of energy storage technologies to accelerate the deployment of storage as a cost-effective technology to support the transition of the grid to a modern electric infrastructure with a low carbon footprint. Part of the ESS Program is a systems analysis element, supporting the core engineering and development elements of the program and addressing the technical, economic, and policy challenges of deploying and integrating storage technologies. Integral to this analysis is this National Assessment of Grid-Connected Energy Storage: Phase 1, WECC, (hereafter referred to as the National Assessment) that attempts to estimate the potential market size for grid-connected energy storage in two distinct markets and for two distinct applications: 1) the energy balancing application, and 2) energy arbitrage. While many other individual grid benefits can be delivered by energy storage systems, this assessment focuses on the two key storage applications that are large, well-defined, already being targeted by advanced storage vendors, and manageable from a data requirements and analysis point of view (Rastler 2010). This is not to say that applications other than balancing and arbitrage services are less important, or even smaller in size. The choice of the two distinct applications was primarily motivated by the fact that we have some ability to quantify the magnitude of their market potential, whereas others are more difficult to quantify or require highly detailed and infrastructure-specific data.

The National Assessment is the first attempt to estimate the market size on a region-by-region basis, with a total of 22 regions, as defined by the NERC and then further subdivided into sub-regions as defined by the Energy Information Administration (EIA) and the Environmental Protection Agency $\left(\right.$ EPA $^{1}$ (DOE/EIA 2011).

\footnotetext{
${ }^{1}$ http://www.epa.gov/egrid.
} 
The results will be delivered in two Phases: Phase 1 addresses the WECC, Phase 2 includes the ERCOT, and the Eastern Interconnection (EI). The two phases will make up the entire National Assessment of Grid-Connected Energy Storage. Phase 1 includes the methodology of the assessment.

This study evaluates the benefits and market potential of energy storage associated with load balancing services in the WECC. While this is an important service that yields significant value, it is only one in a larger set of services offered by energy storage. Extensive research into a broad spectrum of energy storage value streams conducted by the Sandia National Laboratories, the Electric Power Research Institute and other groups indicates that the market size for energy storage in the U.S. could be significantly greater than the market captured by balancing services alone.

The results of an energy storage and market assessment guide conducted by Eyer and Corey (2010) of the Sandia National Laboratories are presented in Figure 1.1. As shown, the study identified a number of distinct services with benefits ranging from $\$ 86$ per $\mathrm{kW}$ for transmission congestion relief to \$2,400 per $\mathrm{kW}$ for substation on-site power. The U.S. market potential was also estimated for each service. For several of the services, the market size exceeded $15 \mathrm{GW}$ nationally, with time-of-use energy cost management topping the list at $64.2 \mathrm{GW}$.

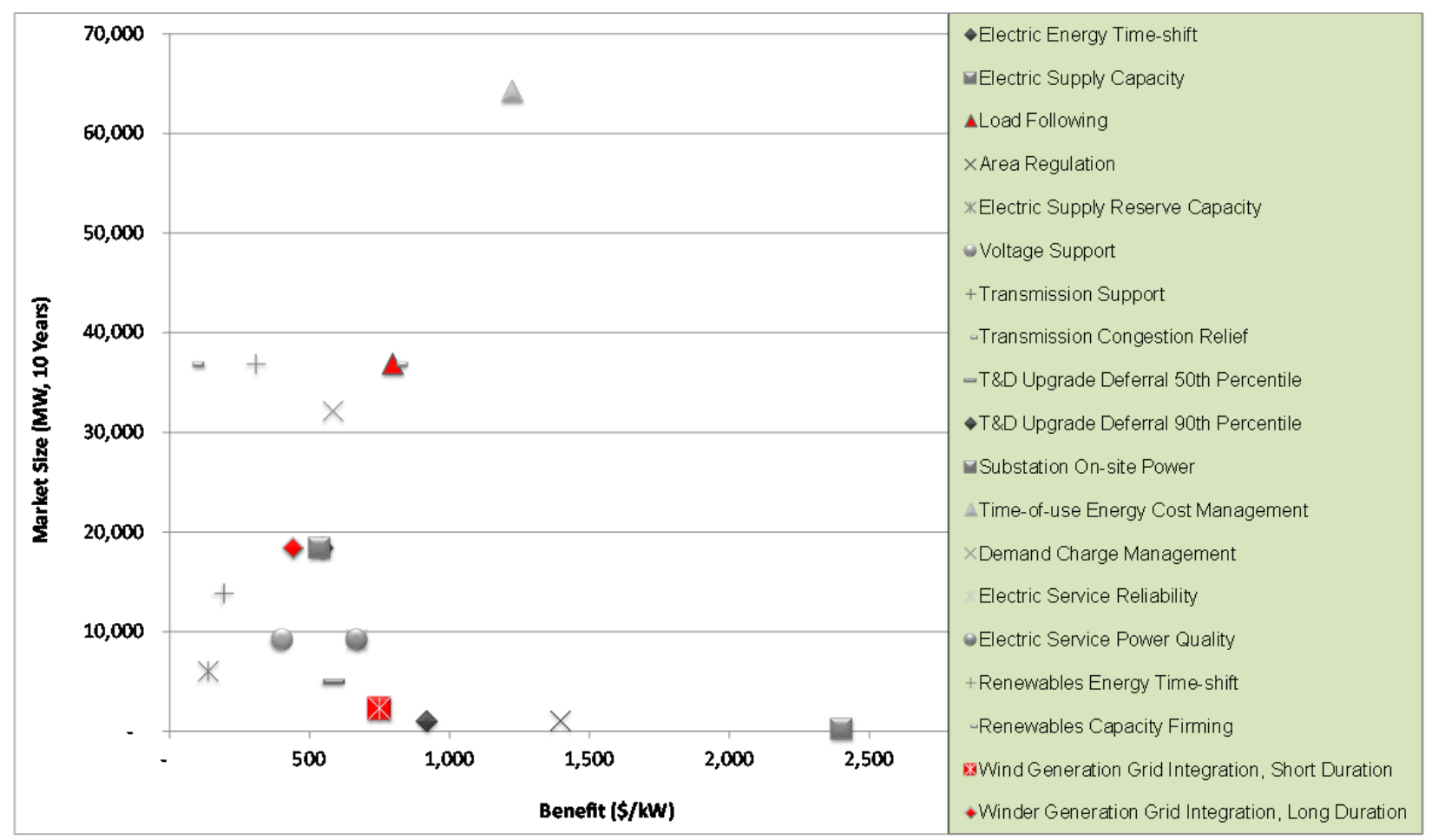

Figure 1.1. Energy storage and market assessment, conducted by Sandia National Laboratories (Eyer and Corey, 2010). 


\subsection{Objectives and Scope}

The objectives of this National Assessment are to address several questions raised in the electricity industry, formulated in a 2010 DOE-sponsored workshop, and summarized in "Electric Power Industry Needs for Grid-Scale Storage Roadmap” (Nexight 2010). The workshop revealed several grid applications of interest for applying energy storage technologies, including: a) area and frequency regulation (short duration), b) renewable integration (short duration), c) transmission and distribution upgrade deferral (long duration), d) load following (long duration), e) electric energy time shift (long duration).

This assessment addresses area and frequency regulation (short duration) and renewable integration in an aggregated form - balancing services. This assessment focuses on imbalances between demand and supply, and spans the entire spectrum of cycles from seconds to minutes. The longer duration applications are captured by analyzing operational benefits of arbitrage strategies that utilize low cost electricity during off-peak periods and dispenses it during high-cost periods during system peak periods. When operating storage in this manner, energy will be time-shifted. The capital cost benefit of deferring infrastructure upgrades are difficult to quantify and are not studied in this assessment. Evaluating infrastructure alternatives would require very specific studies with highly spatially resolved data that considers distribution system or transmissions system expansions and alternatives, which are highly casespecific. Although the capital deferment benefit of storage is important, it is out of scope for this assessment.

In summary, the assessment will address the following set of questions:

1. What are the additional balancing requirements ${ }^{1}$ necessary to accommodate enhanced wind generation capacity, so as to meet the RPS of about $20 \%$ of the generation for the WECC (in Phase 1) in 2020? This analysis assumes that state-specific RPS above 20\%, such as California's 30\% RPS target for 2020, will be honored. Estimates are derived and discussed for four NERC WECC subregions. These sub-regions are the Arizona-New Mexico-Southern Nevada (AZNM) Power Area, California-Mexico (CAMX) Power Area, NWPP, and RMPA. Phase 2 will focus on the balancing requirements for the ERCOT and the Eastern Interconnect.

2. What are the most cost-effective technology options for providing additional balancing requirements? Our analysis includes the following technologies:

i. Combustion turbine as a base case technology

ii. Na-S (Sodium Sulfur) batteries

iii. Li-ion (Lithium-ion batteries)

iv. Flywheel

v. CAES

\footnotetext{
${ }^{1}$ A balancing market is a niche market within a competitive electricity market for last-minute, just-in-time, rapidresponse electricity. This market may demand either increases or decreases in a quantity of electric power. Electricity generators are paid to quickly ramp up or ramp down their electric power in this market. This market results from discrepancies between scheduled electric power generation and actual real-time electric demand. This market is often served by fast-ramping electric power plants like gas turbines and by demand response.
} 


\section{vi. Redox Flow batteries}

vii. Pumped Hydroelectric (PHES) Storage

viii. Demand Response

ix. Hybrid energy storage systems (configurations of various above mentioned storage technologies)

3. What is the market size for energy storage and its respective cost target for balancing and energy arbitrage applications by regions?

The questions above address the two time scales in which storage is usually applied: short duration, which requires storage capacities for 15-30 minutes, and long duration storage that provides charging or discharging capabilities at rated capacity for several hours (e.g., 4-10 hours, or potentially more).

As a National Assessment, the study needs to be broad in scope - providing a meaningful picture of the opportunities and potential market sizes from a national perspective - while still providing sufficient resolution to consider some of the regional specifics that drive the results. For instance, wind resources are non-uniformly distributed throughout the United States. Furthermore, existing available generation capacities and their generation mix vary across the regions and load profiles vary in accordance to populations, economic activities, and climate conditions. To consider some of these key drivers suggested an assessment by regions (Figure 2.1). A 22-region envelope provided sufficient spatial resolution to capture the distribution and diversity of the wind resource potential, the load profiles and existing installed generation capacity, and the inter-regional transfer limits within the bulk power transmission network. 


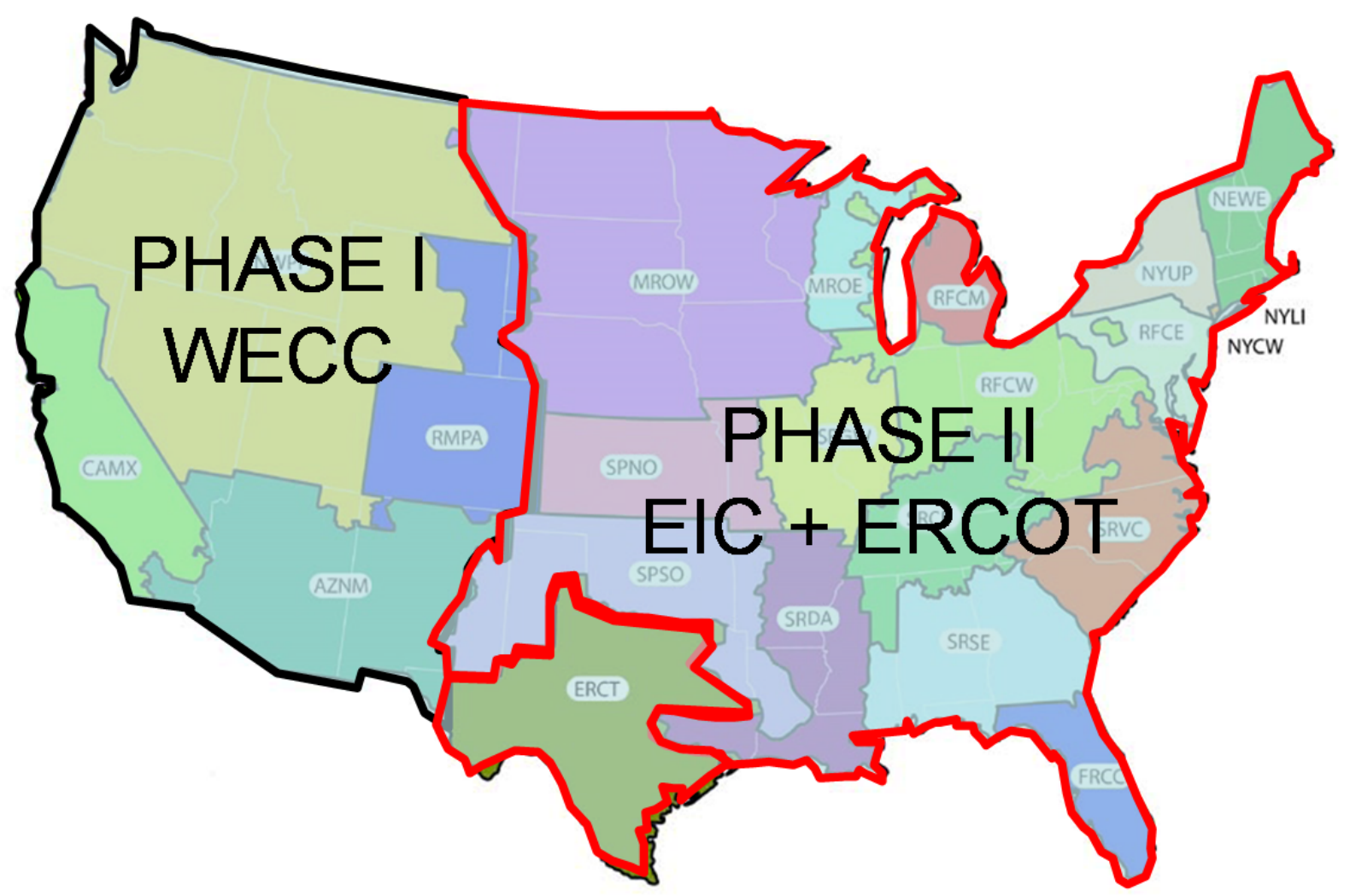

Figure 2.1. Spatial Definition of Regions based on NERC-Regionalization (DOE/EIA 2011)

\section{Legend:}

1 - Texas Reliability Entity (ERCT)

2 - Florida Reliability Coordinating Council (FRCC)

3 - Midwest Reliability Organization / East (MROE)

4 - Midwest Reliability Organization / West (MROW)

5 - Northeast Power Coordinating Council / New England (NEWE)

6 - Northeast Power Coordinating Council / NYC-Westchester (NYCW)

7 - Northeast Power Coordinating Council / Long Island (NYLI)

8 - Northeast Power Coordinating Council / Upstate New York (NYUP)

9 - ReliabilityFirst Corporation / East (RFCE)

10 - ReliabilityFirst Corporation / Michigan (RFCM)

11 - ReliabilityFirst Corporation / West (RFCW)

12 - SERC Reliability Corporation / Delta (SRDA)

13 - SERC Reliability Corporation / Gateway (SRGW)

14 - SERC Reliability Corporation / Southeastern (SRSE)

15 - SERC Reliability Corporation / Central (SRCE)

16 - SERC Reliability Corporation / Virginia-Carolina (SRVC)

17 - Southwest Power Pool / North (SPNO)

18 - Southwest Power Pool / South (SPSO)

19 - WECC / Southwest, Arizona and New Mexico (AZNM)

20 - WECC / California and Mexico (CAMX)

21 - WECC / NWPP

22 - WECC / RMPA 



\subsection{How is this Assessment Different from Other Studies?}

This National Assessment fills an essential gap in the analysis landscape of grid-connected energy storage and generation. Early in the scoping discussion of the National Assessment, it was decided that this Assessment would provide the most value by focusing on modeling and analysis depth with sufficient breadth to address the fledging stationary storage industry. Prior studies have chosen to explore the values of energy storage in all of its various application areas with an emphasis on being comprehensive in breadth. These studies have evaluated various sub-segments of the electricity market and the variety of sources of financial value garnered from grid connection. The methodologies emphasized either (1) a literature review of what other organizations had published already or (2) economic analysis, generally without thorough computer simulations of the physics of the grid and underlying current and future storage and generation technologies. Some grid operators have performed thorough grid simulations to quantify the regulation and ramping services (what is termed in this report as "balancing services" includes both regulation and ramping services). Most notably are the studies by the Midwest Independent Transmission System Operator (MISO), the California Independent System Operator (CAISO), and the Bonneville Power Administration (BPA). Furthermore, Southern California Edison (SCE) has performed screening studies and economic analytics for both distributed energy storage and central plant (megawatt (MW)-sized) storage applications. These studies were regionally defined by their specific service area and did not provide comprehensive U.S.-wide scenarios that were based on common assumptions across the entire U.S. electricity infrastructure.

The National Assessment looks out to the 2020 time horizon and provides an evaluation of the potential market sizes by 22 regions for future storage and generation technologies for two specific subsegments of the electricity market - the balancing market and the arbitrage market. The underpinnings of this assessment are model-based using a suite of specialty models that focus on specific drivers for this assessment. Furthermore, this analysis researched one of the most sensitive input variables to this modeling work, namely the incremental cost of energy storage and generation technologies, both for today and projected into the future. These costs were researched in depth with approximately 100 citations to the literature and personal conversations with leading industry professionals and leaders in the research communities. Also, unlike prior studies, costs were differentiated according to the applications, with balancing service more strongly influenced by the costs of achieving a high rate of electricity transfer per unit time (i.e., the cost per MW), and arbitrage services more greatly influenced by the cost of storing a certain quantity of total energy (i.e., the cost per MWh).

To provide an overview of how this National Assessment differentiates itself from other studies in the growing storage analysis landscape, we developed Table 3.1 that characterizes the studies by their depth (i.e., the detailed development and deployment of models describing the physics and economics of energy systems) and by their breadth (i.e., extent of market sub-segments covered). The columns indicate different studies conducted. These are referenced in the references section. The rows of the table indicate key differentiating factors of these studies. The color 'green' indicates that a study covers application area or applied a particular methodology. 'Red' means that the study did NOT cover this subject. Detailed descriptions of each of the seven storage studies are presented in Appendix D. 


\begin{tabular}{|c|c|c|c|c|c|c|c|c|}
\hline & Key Differentiating Factors & (1) PNNL 2012 & $\begin{array}{l}\text { (2) EPRI } 2010 \& \\
2012\end{array}$ & (3) MISO 2011 & $\begin{array}{l}\text { (4) Sandia 2002, } \\
\text { 2004, 2008, 2010 }\end{array}$ & $\begin{array}{c}\text { (5) Southern } \\
\text { California Edison }\end{array}$ & (6) KEMA 2010 & \begin{tabular}{|c|} 
(7) Vosen 1999, \\
Lemofouet 2006, \\
Lukic 2006, Henson \\
2008 \\
\end{tabular} \\
\hline & Depth of Modeling & & & & & & & \\
\hline & Stochastic model to determine balancing requirements: & & & & & & & \\
\hline & Wind/load uncertainties & & & & & & & \\
\hline & Diversity due to spatial relations & & & & & & & \\
\hline & Energy Storage Cost Characterization & & & & & & & \\
\hline & $\begin{array}{l}\text { Extensive literature search and industry analysis on } \\
\text { capital cost of storage technol ogies }\end{array}$ & & & & & & & \\
\hline & Estimate minimum and maximum values for year & & & & & & & \\
\hline & $\begin{array}{l}2020 \text { projected costs } \\
\text { Market size in MW and MWh }\end{array}$ & & & & & & & \\
\hline & Storage Sizing Model: & & & & & & & \\
\hline & Differentiation between $\mathrm{MW}$ and $\mathrm{MWh}$ sizing & & & & & & & \\
\hline & approach for Balancing & & & & & & & \\
\hline & $\begin{array}{l}\text { Hybrid system - cost optimizes Life Cycle Costs } \\
\text { Considers energy storage cycle life and efficiency }\end{array}$ & & & & & & & \\
\hline & Ramp rates & & & & & & & \\
\hline & Plethora of technologies considered - DR, $\mathrm{PH}$ & & & & & & & \\
\hline & Arbitrage: & & & & & & & \\
\hline & Production cost modeling considering: & & & & & & & \\
\hline & Transmission congestion & & & & & & & \\
\hline & Existing and future generation & & & & & & & \\
\hline & Efficiencies storage & & & & & & & \\
\hline & Breadth of Applications & & & & & & & \\
\hline End-user & Power quality & & & & & & & \\
\hline & Power reliability & & & & & & & \\
\hline & Retail TOU Energy Charges & & & & & & & \\
\hline & Retail Demand Charges & & & & & & & \\
\hline Distribution & $\begin{array}{l}\text { Voltage support } \\
\text { defer distribution investment }\end{array}$ & & & & & & & \\
\hline & distribution loss & & & & & & & \\
\hline & VAR support & & & & & & & \\
\hline Transmission & Transmission congestion & & & & & & & \\
\hline fransmission & transmission access charges & & & & & & & \\
\hline & defer transmission investment & & & & & & & \\
\hline System & $\begin{array}{l}\text { local capacity } \\
\text { system capacity }\end{array}$ & & & & & & & \\
\hline & renewable energy integration & & & & & & & \\
\hline & $\begin{array}{l}\text { fast regulation (1 hour) } \\
\text { regulation (1 hour) } \\
\text { regulation (15 min) }\end{array}$ & & & & & & & \\
\hline ISO markets & spinning reservess & & & & & & & \\
\hline & $\begin{array}{l}\text { non-spinning reserves } \\
\text { black start }\end{array}$ & & & & & & & \\
\hline & price arbitrage & & & & & & & \\
\hline
\end{tabular}

Table 3.1. Characterization of Major Storage Studies

Covered by analysis:

Not covered by analysis:

(1) This document; (2) EPRI (2009), Rastler (2010, 2011a); (3) MISO (2011), Rastler (2011b); (4) Butler (2002), Eyer (2004), Schoenung (2008), (Eyer 2010);

(5) Ritterhausen (2011); (6) KEMA (2010); and (7) various papers on hybrid storage systems 


\subsection{Technology Choices for Balancing Services}

\subsection{Introduction}

To mitigate the additional intermittency and fast ramps at higher penetration of intermittent energy resources (i.e., wind power in our case) in the NWPP, the conventional solution is to build more peaking units such as combustion turbine units. However, the advancement of battery technology, smart grid concepts coupled with demand response options, and the anticipated need for carbon reduction, places new emphasis on exploring non-conventional resources. The importance of a broader set of technology options for providing the ancillary services requirements that traditionally have been provided by faststarting and flexible CT cannot be understated.

The following technologies are considered for this study:

- CT, as the base case technology

- Sodium sulfur (Na-S) battery

- Lithium-ion (Li-ion) battery

- Vanadium reduction-oxidation (redox) flow battery

- CAES

- Flywheels

- PHES

- Demand response.

This following section provides a high level overview of the above technologies. A detailed discussion on the cost and performance characteristics of battery technology considered is provided in Appendix A.

\subsection{Technology Overview}

\subsubsection{Combustion Turbine}

CT, as applied in this study, are designed to provide an output of about $160 \mathrm{MW}$ while operating at an energy efficiency of 31.5\%. The efficiency is expressed in terms of a heat rate of 10,833 British Thermal Units per kilowatt-hour (Btu/kWh) at full load condition. The heat rate increases with lower partial load conditions (DOE/EIA 2008). In 2019, combustion turbine capital costs are estimated in the 2011 Annual Energy Outlook (AEO) at $\$ 990$ per kW (DOE/EIA 2010b). The economic life of the CT is estimated to be 15 years.

\subsubsection{Combined Cycle Plant}

Although the combined cycle plant is not directly applied as a technology option for providing balancing services, it provides the electric energy fed through the energy storage system. It is, thus, the 
energy provider on the margin that makes up for the energy losses in the storage device. The cost for fuel, O\&M, and emissions associated with the energy lost in storage are considered in the LCC analysis.

The typical size of a combined cycle power plant is about 250-300 MW. The design heat rate is commonly cited as 7,196 Btu per kWh (DOE/EIA 2008). The efficiency of a combined cycle power plant is generally higher than that of a combustion turbine. Typical design efficiencies are approximately $47 \%$. More details regarding cost assumptions underlying both CT and combined cycle power plants are presented in Section 5.0, Energy Storage Technologies.

The largest Na-S battery system tested is a 34-MW battery system installed in Rokkasho village in Aomori, Japan (NGK Insulators, LTD), while the corresponding number for Li-ion system is $2 \mathrm{MW}$ (KEMA 2008). A 12-MW energy storage system has been installed by AES Energy Storage using Li-ion batteries supplied by A123 Systems (Parker 2010). PH systems are available in the order of hundreds of MW and MWh.

Of the three energy storage technologies considered, PH energy storage is technologically most matured. The table below provides a perspective of the level of maturity based on installed capacity of grid-connected storage in the U.S. and globally (Nourai 2009).

Table 4.1. Installed Capacity for Various Energy Storage Devices in the U.S. and Worldwide

\begin{tabular}{lrc}
\hline & U.S. (MW) & Global (MW) \\
\hline Pumped Hydro & 23,000 & 110,000 \\
Compressed Air & 110 & 477 \\
Batteries & 40 & 300 \\
Other & 5 & 10 \\
\hline
\end{tabular}

Table 4.1 suggests that there is significant room for cost and performance improvements of the less mature technologies (compressed air and batteries), while PH technologies, due their maturity, are not likely achieve cost reduction - at least, at the same rate possible with the nascent battery technologies.

\subsubsection{Sodium Sulfur Battery}

The response time for both Na-S and Li-ion battery systems is in the order of a few milliseconds (Divya and Østergaard 2009). This allows the systems to provide power almost instantaneously, as demanded by the grid. While numbers as high as $90 \%$ have been used for battery efficiency, it is important to use appropriate efficiency values that correspond to power needs for various applications. It would also be reasonable to take into account battery degradation as a function of calendar and cycle life to incorporate losses in specific power/energy, power/energy density and efficiency losses as battery state of health degrades. For this analysis, a system efficiency of $78 \%-80 \%$ was used for both battery systems. This value also includes efficiency losses from the power conversion system (PCS).

Currently, commercially available Na-S batteries are designed to discharge over periods as long as 7 to 10 hours (Kamibayashi et al. 2002; Nourai 2007). When these batteries are used for very small durations (in the order of seconds to minutes), the batteries can provide power as high as five times the 
rated power; where the rated power is defined as power for a 7-hour discharge (Kamibayashi et al. 2002). For this study, peak power occurs for only 1 to 2 minutes, hence the required power rating of the battery that is needed could be as low as $1 / 5$ of the peak power. At present, Na-S batteries are commercially available in an energy/rated power (E/P) ratio of 6-7. For this study, it has been assumed that in the future, batteries with E/P as low as 1 will be available to avoid over-sizing the batteries.

\subsubsection{Lithium-ion Battery}

Li-ion batteries are available from various sources. A 2-MW battery from AES Energy Storage, with the battery supplied by Altairnano, was tested under the direction of KEMA recently (KEMA 2008; Altair Nanotechnologies 2008). During the test, a battery management system monitored battery cell temperatures, balanced cell voltage, and kept track of battery state of charge. Three single-phase Parker Hannifin SSD power inverters were coupled to isolation transformers and fed into a step-up transformer, with the battery side running at $480 \mathrm{~V}$ and the grid side at $13.8 \mathrm{kV}$. These voltage values are important, since the capital cost of PCS depends on the minimum voltage at the battery side, as will be discussed later. These batteries performed well and dispatched power almost instantaneously. It remains to be seen how they would hold up over the long-term, and what the impact would be of connecting several batteries in series/parallel configuration to provide the required output voltage and power.

\subsubsection{Pumped Hydro Energy Storage}

PH energy storage technology has been used for various utility applications. One of its limitations is the need to wait prior to reversing direction from charge to discharge. Variable speed pumps/turbines allow better control of ramp rates, but cost more. A detailed discussion of $\mathrm{PH}$ is provided in Appendix A.

The response time for PH systems is fast achieving high ramp rates of $3000 \mathrm{MW} / \mathrm{min}$, which corresponds to 3\% rated capacity per second (First Hydro Company 2009); calendar life is estimated to be 50 years (Schoenung 2001). There is, however, a waiting period of several minutes every time the operating mode changes. This is necessary due to significant inertia in the turbine and the hydro dynamics in and above the turbine. To meet the balancing requirements, two operating design options are investigated. The first option emulates the operation of a battery system that permits rapid changes between charging and discharging modes in accordance to the balancing requirements. Advancements in the turbine/pump design allows for frequent mode change between pumping (charging) and generating (discharging) modes. However, because of the significant hydrodynamic and mechanical inertia in the turbine, a delay of significant duration is required. The estimated 4-minute delay is of sufficient significance for meeting the balancing requirements, causing the back-up resource to be sized quite large. This delay renders the machine temporarily inoperable. Thus, some additional resources must be assigned during that period as a 'back-up' resource. A Na-S battery was chosen to function as a back-up resource.

The alternative and more commonly observed operation of pumped-hydro storage is a 2-mode operating schedule, whereby the machine is operated in a pumping mode during the off-peak hours and in a generating mode during the day. While in either of the modes, the machine can meet the balancing requirements. However, the pump/generator size must be upsized compared to 'multiple mode changes' mode because the balancing requirements must be met in both pumping and generating modes individually. This requires a pump/generator size that covers the entire swing from full increment to full 
decrement. A very small Na-S battery is applied as a back-up resource to meet the balancing requirements during the 2-mode change.

\subsubsection{Compressed Air Energy Storage}

CAES uses electricity to compress air using an electric motor and store it either in underground caverns or above ground vessels or pipes (Rastler 2011; Cavallo 2007). Underground storage comprises various geologic formations such as salt caverns, rock mines, depleted oil and gas reservoirs and aquifers, and is suitable for hundreds of MW of storage for several hours (Ridge 2005). Generation of electricity is carried out by expansion of the compressed air through a series of turbine-generators. Conventional or diabatic CAES burns natural gas in a combustion turbine, thus generating power. The exhaust from the CT is used to heat the compressed air, which is expanded through a high pressure and low pressure turbine (Drury et al. 2011). In a variation of this, the compressed air is heated with natural gas without generation of electricity. There are variations on the CAES concept that include thermal energy storage, operation in the adiabatic mode or isothermal mode with elimination of the combustion turbine, or direct coupling of the compressor with wind turbines to avoid conversion of mechanical energy from the turbines to electricity (Grazzini and Milazzo 2008). The overall efficiency for diabatic CAES is $\sim 0.5$, while it is anticipated that efficiency as high as 0.7 can be obtained for adiabatic/isothermal CAES (Drury et al. 2011). One of its limitations is the need to wait for a few minutes prior to reversing direction from charge to discharge or vice versa. Ramp rates in the range of $15 \%-40 \%$ of rated power per minute have been obtained. A more detailed discussion of these parameters, along with capital and O\&M costs, is given in Appendix A.

\subsubsection{Flywheels}

Flywheels consist of rotors made of steel or carbon composites and store energy in the form of kinetic energy proportional to the mass and the square of the angular velocity of the rotor. The motor/generator set is coupled to the same shaft to which the rotors are attached, with rotation occurring in vacuum to minimize losses. The rotors can be supported by passive or electro-magnetic bearings, with high temperature superconducting magnetic bearings requiring cryogenic cooling providing the best performance (Bolund 2007). While rotors made of graphite fiber composites are currently used, carbon nanotubes are expected to increase the energy density by an order of magnitude over carbon fiber winding (Sibley 2011a). The efficiency of the flywheel systems ranges from $85 \%-90 \%$, while their ramp rate is as high as $100 \%$ of rated power per minute. A more detailed description is provided in Appendix A.

\subsubsection{Vanadium Redox Flow Batteries}

Redox flow batteries were developed in the seventies, and have gained prominence recently due to their flexibility of use (Herman 2003; Rastler 2010). Similar to a regenerative fuel cell, the power and energy components are separated, with the stack providing power and the electrolyte storage tanks providing energy. Vanadium redox flow batteries with a wide range of power and energy capacity in the kW-MW and kWh-MWh have been deployed. The efficiency of these systems is in the $70 \%-80 \%$ range with response time in the order of milliseconds. The stacks last at least 10 years and can sustain $>200,000$ cycles. Active research is ongoing to reduce stack costs by addressing individual components and also by increasing the power density. A more detailed description is provided in Appendix A. 


\subsubsection{Demand Response}

Demand response (DR) is an unused resource fully capable of providing balancing services. Similar to a generator that provides balancing services, a load customer who operates up and down from an original operating point creates a balancing reserve value. In fact, $\mathrm{PJM}^{\circledR}$ allows large load customers to participate in the regulation services markets. Small loads such as residential and commercial customers can also deliver these services to the grid. The challenge is how to coordinate a large number of small devices to operate in a coordinated fashion such that they deliver value reliably at a sufficient scale. Communications technology and smart grid control strategies may advance the access and, thus, the utilization of small residential and commercial end-use devices to deliver value to the grid.

For the purpose of this study, plug-in hybrid EVs (PHEVs) are selected as the key candidates for this service, recognizing that other appliances may contribute as well at certain times. PHEV are not currently mass-produced, and it will take some time for the PHEVs and other EVs to gain market share to amount to a sizable load. However, significant efforts in standardizing the communication to the vehicles are underway to enable smart charging strategies. This would make EVs a likely candidate for providing balancing services.

Figure 4.1 shows the balancing signal and the load resource availability of EVs. The balancing would be achieved solely during the charging mode. No Vehicle-to-Grid (V2G) is necessary to meeting the balancing requirements. PNNL coined the term "V2Ghalf”, expressing the feature of intelligent or smart charging whereby the balancing is provided by a load resource (i.e., charging of a EV/PHEV battery) in such a manner that the charging is varied around an operating point. The aggregated EV battery charging load is not constant but varies as a function of time-of-day and availability of public charging stations at the workplace to allow for making the vehicle resource available to grid services. The number of vehicles necessary to provide sufficient load resources is then the number of vehicles that will furnish just enough load to meet the maximum balancing capacity, as seen in Figure 4.1, at 6:00 a.m. when most of the chargers are turned off after having recharged the battery overnight. More specific information on how the EV resource can be deployed is found in the Appendices and in Tuffner 2011.

\subsection{Technology Cost and Performance Characteristics}

\subsubsection{General Discussion}

Energy storage devices, unlike electric generators, have two capability ratings: 1) the power rating, expressed in kW or MW and the energy rating, expressed in kWh or MWh. This poses a challenge when comparing energy storage equipment with generators, which generally are not energy limited. To fully describe the incremental cost of an energy storage device, generally two specific cost indices must be used: cost per unit power $(\$ / \mathrm{kW})$ and cost per unit energy $(\$ / \mathrm{kWh})$. The literature is relatively inconsistent in this regard. Often, battery devices are characterized by their incremental cost per unit energy ( $\$ / \mathrm{kWh}$ ) masking the cost associated with the balance of plant (BOP) and power conditioning system, which is scaled by the unit of power, or $\$ / \mathrm{kW}$. The costs for PH projects are most commonly specified in $\$ / \mathrm{kW}$, with a cost of $\$ 10 / \mathrm{kWh}$ assigned to the energy component. In most cases, it is determined by the topology of a given location, which sets the size of the reservoir. The same applies to CAES also, with a nominal cost assigned to reservoirs for compressed air storage. For flywheels and redox flow batteries, independent costs per unit power and energy have been provided. 


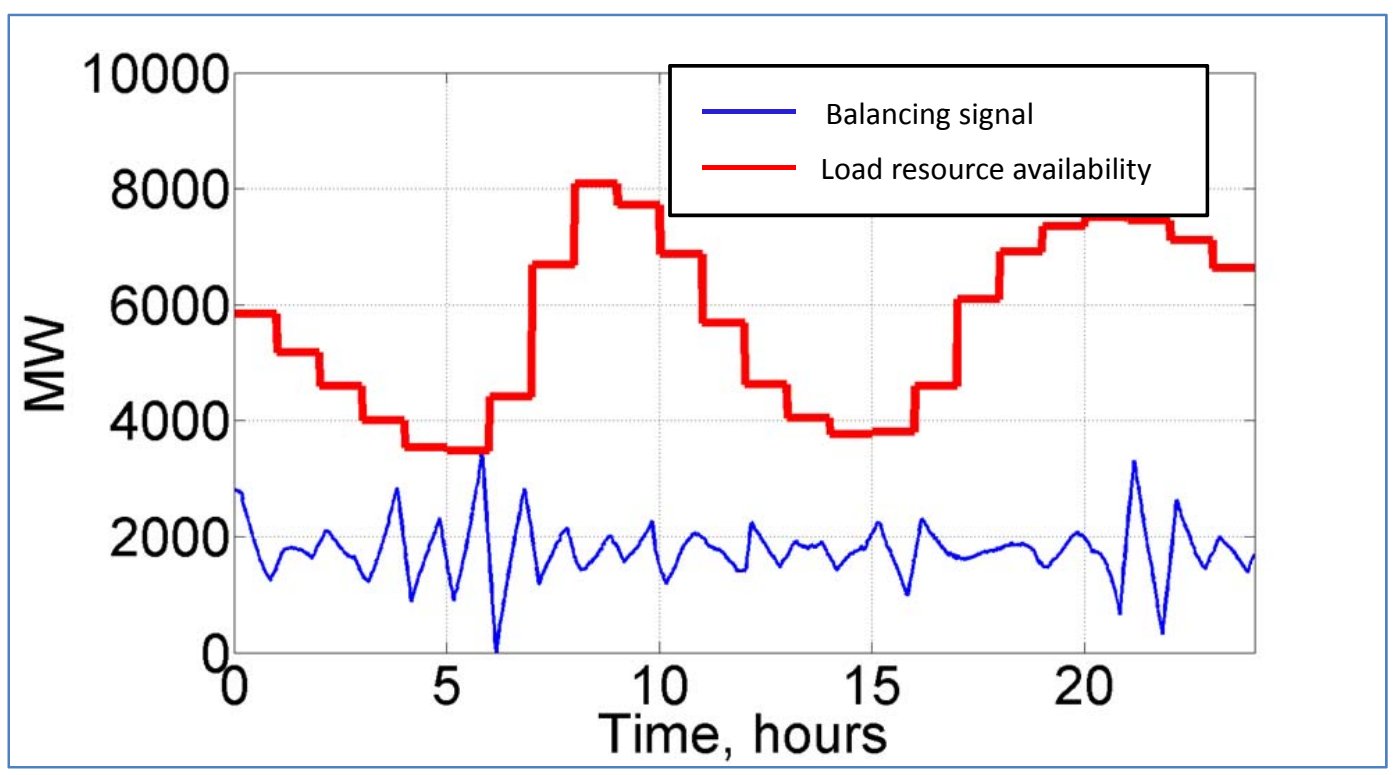

Figure 4.1. Balancing Signal and Load Resource Curve for PHEV with Home and Work Charging ${ }^{1}$

For the purpose of this report, a methodology of describing the incremental cost of energy storage devices was used that reveals both the cost that scales with the power rating $(\$ / \mathrm{kW})$, and the cost that scales with energy content of the device $(\$ / \mathrm{kWh})$.

To determine the capital costs, the energy storage device has to be sized based on the power and energy needs of the application. This means that the capital cost has two components: one that scales with unit power $(\mathrm{kW})$ estimating the cost for the PCS, and the other that scales with unit energy $(\mathrm{kWh})$, which estimates the cost associated with the storage component of the system. To provide any incremental cost, either nominated in $\$ / \mathrm{kW}$ or in $\$ / \mathrm{kWh}$, one needs to estimate the total system cost and then and only then can the incremental cost be derived. However, it should be noted that it is meaningless and often misleading to compare two storage technologies for totally different application on the basis of one incremental cost.

The battery costs are typically given in $\$ / \mathrm{kWh}$, which can be converted to $\$ / \mathrm{kW}$, where the $\mathrm{kW}$ is rated power of the battery. For example, if for a $1 \mathrm{~kW}, 4 \mathrm{kWh}$ system, $\$ 1000 / \mathrm{kW}$ corresponds to $\$ 250 / \mathrm{kWh}$.

Typically, rated power is continuous power, and is defined as power that can be sustained for at least 15 minutes. Peak power typically is defined as pulse power for 2-second duration. The ratio of peak power/rated power is a function of battery chemistry and design.

\footnotetext{
${ }^{1}$ Based on simulations using the USDOT 2001 National Household Travel Data (DOT 2003) and applying smart charging strategies that assures that vehicle batteries are fully charged at the time when owner leaves (Tuffner 2011).
} 


\subsubsection{Summary of Capital, O\&M Costs, and Efficiency for Batteries and PH Systems}

Based on the values obtained from an extensive literature review and through many consultations with domain experts, Table 4.2 summarizes the values used in this study, with 2020 values within parentheses. The range of capital costs for years 2011 and 2020 are provided in Table 4.3. More detailed cost and performance characteristics as well as ranges of cost as found in the literature are included in Appendix A. The TRL and manufacturing readiness level (MRL) for each energy storage option are also included. TRL1 represents a technology still at the basic research level, while TRL9 indicates the system is ready for full commercial deployment. All the energy storage options included in this report are at least at the prototype level TRL6, with Na-S, Li-ion, and flywheels performance validated in various demonstration projects (TRL7). Pumped hydro systems are the most widely deployed and have been assigned the highest TRL of 9, while redox flow batteries are assigned the prototype level of TRL6. The MRL assignments closely track the TRLs assigned to each storage system. MRL1 corresponds to feasibility assessed stage, while MRL10 indicates the technology has ramped up to full rate production. CTs and combined cycle (CC) systems have been assigned MRL10, while PH and CAES systems have been assigned MRL7. This is due to the site-specific consideration that has to be taken into account, thus adding a layer of complexity. Li-ion batteries have been assigned MRL6, with the expectation that with higher penetration of battery powered vehicles, this level will increase to MRL9 in about 2 years. Na-S batteries currently are manufactured by only one vendor, and hence have been assigned MRL6. For flywheels, rotors made of novel materials such as graphite and carbon nanotubes are expected to be incorporated to increase energy density and life, while reducing cost. Redox flow batteries, while being demonstrated at various sites, also have room for improvement in terms of materials selection and cell design. Both flywheels and redox flow batteries have been assigned MRL5. Details of the TRL and MRL descriptions are provided in Appendix A, Section A.7.

This study assumes that technologies with low TRL and MRL scores have further room for improvement in cost reduction, which are reflected in lower 2020 costs relative to 2011 costs.

\subsubsection{Explanation for Cost Spread}

The cost for installed Na-S systems has been provided in terms of $\$ / \mathrm{kW}$, with the cost ranging from $\$ 2400-\$ 4300 / \mathrm{kW}$ (Nourai 2007; Kishinevsky 2006; NGK 2007). The battery cost is estimated to be $50 \%$ of the installed cost. For 2011 costs, the battery costs, including non-recurring engineering support, are estimated to be $80 \%$ of installed costs, while for 2020, the battery costs are expected to be $50 \%$ of current installed costs. Additional details are provided in Appendix A, Section A.1.2.

For compressed air, the range of costs from various sources in the time frame 2003 to 2011 was $\$ 300-\$ 1200 / \mathrm{kW}$, with the cost increasing with the time when costs were given. In the time frame 2007-2010, most costs were in the \$800-\$850 range, with one estimate being \$1140/kW in 2011 excluding storage costs. Hence for year 2011, the range was set at $\$ 850-\$ 1140$. For year 2020, some advanced low cost concepts proposed (Nakhamkin 2008, 2009, 2010) were also considered, with the range set at $\$ 500-\$ 1140 / \mathrm{kW}$.

For flywheels, costs are reported as Total $\$ / \mathrm{kW}$. As discharge time increases, Total $\$ / \mathrm{kW}$ would increase. In the time frame $1999-2011$, a wide range of $\$ 250-\$ 3000 / \mathrm{kW}$ has been reported. The unit energy cost using steel rotors is $\$ 148 / \mathrm{kWh}$, while carbon rotors cost $\$ 350-\$ 380 / \mathrm{kWh}$. The current total 
cost for a 1-MW, 250-kWh system from Beacon Power is $\$ 1000 / \mathrm{kW}$, which corresponds to a unit power cost of $\$ 865 / \mathrm{kW}$ assuming $\$ 148 / \mathrm{kWh}$ for the energy component. A range of $\$ 965-\$ 1590 / \mathrm{kW}$ has been set for current unit power costs, with a unit energy cost of $\$ 148 / \mathrm{kWh}$ using steel rotors. For the year 2020, based on literature and telephone conversations, the range was set at $\$ 200-\$ 865 / \mathrm{kW}$, with a unit energy cost range of $\$ 81-\$ 148 / \mathrm{kWh}$ (lower cost corresponding to graphite fiber rotors).

Table 4.2. Summary of Capital and O\&M Costs for Technologies Analyzed. Note values are representative for 2011 technologies. 2020 values are in parentheses.

\begin{tabular}{|c|c|c|c|c|c|c|c|c|c|}
\hline Parameter & Na-S Battery & $\begin{array}{l}\text { Li-ion } \\
\text { Battery }\end{array}$ & $\begin{array}{l}\text { Pumped } \\
\text { Hydro }\end{array}$ & $\begin{array}{l}\text { Combustion } \\
\text { Turbine }\end{array}$ & $\mathrm{CC}$ & $\begin{array}{l}\text { Demand } \\
\text { Response }\end{array}$ & CAES & Flywheel & $\begin{array}{c}\text { Redox } \\
\text { Flow } \\
\text { Battery }\end{array}$ \\
\hline $\begin{array}{l}\text { Technology } \\
\text { Readiness } \\
\text { Level (TRL) }\end{array}$ & 7 & 7 & 9 & 9 & 9 & 6 & 8 & 7 & 6 \\
\hline $\begin{array}{l}\text { Manufacturing } \\
\text { Readiness } \\
\text { Level (MRL) }\end{array}$ & 6 & 6 & 7 & 10 & 10 & 2 & 7 & 5 & 5 \\
\hline $\begin{array}{l}\text { Battery Capital } \\
\text { cost } \$ / \text { kWh(a) }\end{array}$ & $415(290)$ & $\begin{array}{l}1000 \\
(510)\end{array}$ & 10 & & & & 3 & 148 (115) & $\begin{array}{c}215 \\
(131)\end{array}$ \\
\hline $\begin{array}{l}\text { System Capital } \\
\text { cost } \$ / \mathrm{kW}\end{array}$ & & & $\begin{array}{l}1750 \\
(1890)\end{array}$ & 1009 (990) & Not used & 620 & $\begin{array}{l}1000 \\
(850)\end{array}$ & $\begin{array}{l}1277 \\
(610)\end{array}$ & $\begin{array}{l}1111 \\
(775)\end{array}$ \\
\hline PCS $(\$ / k W)$ & $220(150)$ & $\begin{array}{c}220 \\
(150)\end{array}$ & & & & & & & $\begin{array}{c}220 \\
(150)\end{array}$ \\
\hline $\mathrm{BOP}(\$ / \mathrm{kW})$ & $85(50)$ & $85(50)$ & & & & & & $85(50)$ & $85(50)$ \\
\hline $\begin{array}{l}\text { O\&M fixed } \\
\$ / k W \text {-year }\end{array}$ & 3 & 3 & 4.6 & 10.24 & 14.93 & & 7 & 18 & $\begin{array}{l}39.5 \\
(5)\end{array}$ \\
\hline $\begin{array}{l}\text { O\&M fixed } \\
\$ / k W-y e a r \\
(\mathrm{PCS})\end{array}$ & 2 & 2 & & & & & & & 2 \\
\hline $\begin{array}{l}\text { O\&M variable } \\
\text { cents/kWh }\end{array}$ & 0.7 & 0.7 & 0.4 & 0.9 & 0.4 & & 0.3 & 0.1 & 0.1 \\
\hline $\begin{array}{l}\text { Round trip } \\
\text { efficiency }\end{array}$ & 0.78 & 0.80 & 0.81 & 0.315 & & & 0.50 & 0.85 & 0.75 \\
\hline
\end{tabular}

Table 4.3. Table Range of Capital Costs for Years 2011 and 2020

\begin{tabular}{lcccc}
\hline & \multicolumn{2}{c}{ 2011 Range } & \multicolumn{2}{c}{2020 Range } \\
\cline { 2 - 5 } \multicolumn{1}{c}{ Technology } & $\$ / \mathrm{kWh}$ & $\$ / \mathrm{kW}$ & $181-331$ & $\$ / \mathrm{kW}$ \\
\hline Na-S & $257-491$ & & $290-700$ & \\
Li-ion & $850-1000$ & $1500-2300$ & 10 & $1640-2440$ \\
Pumped Hydro & 10 & $850-1140$ & 3 & $500-1140$ \\
Compressed air & 3 & $965-1590$ & $81-148$ & $200-820$ \\
Flywheel & 148 & $942-1280$ & $88-173$ & $608-942$ \\
Redox flow battery & $173-257$ & &
\end{tabular}


For redox flow batteries, costs were estimated based on currently available estimates for stack components and chemicals, and projected improvements in cost and performance. Our analysis indicated unit power cost range of $\$ 600$ to $\$ 1250 / \mathrm{kW}$ and energy cost of $\$ 80$ to $\$ 260 / \mathrm{kWh}$. The current costs were estimated to be bounded by the mid-point of the above range and the higher end of this range, while 2020 costs were bounded by the lower end of this range and the mid-point of this range.

For Li-ion batteries, the costs estimates in the years 2009 to 2010 were $\$ 850$ to $\$ 1000 / \mathrm{kWh}$, while longer range estimates from the literature were in the $\$ 290$ to $\$ 700 / \mathrm{kWh}$ range.

For $\mathrm{PH}$, in the years $1996-2006$, costs were in the $\$ 600-\$ 1000 / \mathrm{kW}$ range. Conversations with Rick Miller (Renewable Energy Services) and e-mail exchanges indicated cost of \$1500-\$2300/kW.

Accounting for potential technological improvements being offset by increasing material costs and increasing trend of PH system prices, the cost for 2020 was estimated to be $\$ 1640-\$ 2440 / \mathrm{kW}$.

\subsection{Definition of Technology Options}

The set of technologies mentioned above can be applied individually or in combination with other technologies. Technology 'packages' of two technologies are investigated. These technology packages can be thought of as a portfolio of resources - in most cases will be dispersed throughout the four WECC areas. Only in the cases of PH and CAES energy storage would a single location, or potentially multiple locations, be viable based on the topology to support upper and lower reservoirs (for $\mathrm{PH}$ ) or the geological cavities for storing air in the ground (for CAES). For most of technologies, the actual capacity will be widely dispersed. This is particularly the case for demand response. Table 4.4 shows the 16 single technology packages, which we will call 'cases'.

Explored were seven hybrid storage combinations comprised of two technologies. The selection of the pairing was arbitrary and somewhat guided by the intuition that a technology designed for a high power application be complementary and perhaps more cost-competitive when paired with a high energy capacity technology. We defined seven technology pairings (C10 through C16) were studied the cost optimality that minimizes the total LCC. 
Table 4.4. Definition of Technology Cases

\begin{tabular}{|c|c|c|c|}
\hline & Case & Technology & Comments \\
\hline \multirow{9}{*}{ 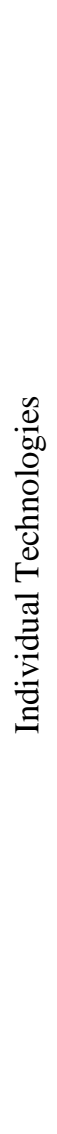 } & $\mathrm{C} 1$ & Combustion turbine & Conventional technology considered as the reference case. \\
\hline & $\mathrm{C} 2$ & $\mathrm{Na}-\mathrm{S}$ & Sodium sulfur battery only. \\
\hline & C3 & Li-ion & Lithium-ion battery only. \\
\hline & $\mathrm{C} 4$ & Flywheel & Flywheel only. \\
\hline & C5 & CAES with 2 mode changes & $\begin{array}{l}\text { CAES with a 7-minute waiting period for mode changes } \\
\text { (compression-generation and vice versa). Balancing services } \\
\text { will be provided during compression mode at night ( } 8 \mathrm{pm}-8 \mathrm{am} \text { ) } \\
\text { and during generation mode during the day ( } 8 \mathrm{am}-8 \mathrm{pm}) . \mathrm{Na}-\mathrm{S} \\
\text { battery is assumed to make up operations during } 7 \text { minute } \\
\text { waiting period. }\end{array}$ \\
\hline & C6 & Flow battery & Flow battery only. \\
\hline & C7 & PH with multiple mode changes & $\begin{array}{l}\text { PH with a 4-minute waiting period for mode changes (pumping- } \\
\text { generation and vice versa). This machine allows multiple mode } \\
\text { changes during the day. Na-S battery is assumed to make up } \\
\text { operations during } 4 \text { minute waiting period. }\end{array}$ \\
\hline & $\mathrm{C} 8$ & PH with 2 mode changes & $\begin{array}{l}\text { Same as (C7), except only two mode changes. Balancing } \\
\text { services will be provided during pumping mode at night ( } 8 \mathrm{pm}-8 \\
\text { am) and during generation mode during the day ( } 8 \mathrm{am}-8 \mathrm{pm}) \text {. } \\
\text { Na-S battery is assumed to make up operations during } 4 \text { minute } \\
\text { waiting period. }\end{array}$ \\
\hline & C9 & DR (demand response) & $\begin{array}{l}\text { Demand response only. This assumes that balancing services } \\
\text { will be provided as a load. Only considered is PHEV charging } \\
\text { at home and work. Resources are expressed in MW of DR } \\
\text { capacity as well as in numbers of PHEV with demand response } \\
\text { capability. }\end{array}$ \\
\hline \multirow{8}{*}{ 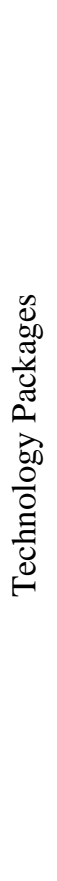 } & $\mathrm{C} 10$ & $\mathrm{Na}-\mathrm{S}$ & Sodium culfur hatterv and $\mathrm{DR}$ combined \\
\hline & & $\mathrm{DR}$ & 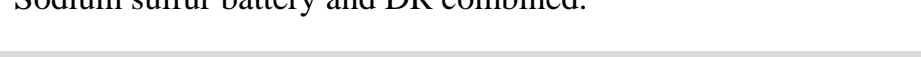 \\
\hline & C11 & $\begin{array}{l}\text { Li-ion } \\
\text { DR }\end{array}$ & Lithium-ion battery and DR combined \\
\hline & C12 & $\begin{array}{l}\text { CAES } \\
\text { Flywheel }\end{array}$ & $\begin{array}{l}\text { CAES with no constraints for mode changes with Flywheel. The } \\
\text { balancing requirement is allocated to each technology according } \\
\text { to minimum cost. }\end{array}$ \\
\hline & C13 & $\begin{array}{l}\text { PH with multiple mode chances } \\
\text { Na-S }\end{array}$ & $\begin{array}{l}\text { PH with no constraints for mode changes with Na-S battery. } \\
\text { The balancing requirement is allocated to each technology } \\
\text { according to minimum cost. }\end{array}$ \\
\hline & C14 & $\begin{array}{l}\text { PH with } 2 \text { mode changes } \\
\text { Na-S }\end{array}$ & $\begin{array}{l}\text { PH with two mode changes per day (see C8) with Na-S battery. } \\
\text { The balancing requirement is allocated to each technology } \\
\text { according to minimum cost. }\end{array}$ \\
\hline & C15 & $\begin{array}{l}\text { PH with multiple mode chances } \\
\text { Flywheel }\end{array}$ & $\begin{array}{l}\text { PH with no constraints for mode changes with Flywheel. The } \\
\text { balancing requirement is allocated to each technology according } \\
\text { to minimum cost. }\end{array}$ \\
\hline & C16 & $\begin{array}{l}\text { PH with } 2 \text { mode changes } \\
\text { Flywheel }\end{array}$ & $\begin{array}{l}\text { PH with two mode changes per day (see C8) with Flywheel. } \\
\text { The balancing requirement is allocated to each technology } \\
\text { according to minimum cost. }\end{array}$ \\
\hline
\end{tabular}




\subsection{Methodology for Estimating Balancing Requirements}

\subsection{Overview of Analysis}

PNNL developed an analytical framework for the National Assessment for the purpose of:

1. Estimating the total balancing requirements associated with forecasting errors both for load and for generation from variable renewable energy resources

2. Sizing grid resources (generation, storage, DR) to meet the new balancing requirements

3. Minimizing the LCC associated with technology options and the economic dispatch to meet the new balancing requirements. The balancing requirements are expressed as a time series of fluctuating power injections (increments) into and power absorptions (decrement) out of the bulk power system on a minute-to-minute basis. Balancing services compensate the over- and under-predictions of scheduled generation to meet the load.

The analytical framework provides a set of sizing tools to dispatch one or several resources to meet the balancing requirements. The resources can be energy storage devices, commonly used generator or DR strategies. Several different dispatch strategies have been developed to dispatch an ensemble of several storage devices or bundled resources comprised of DR, energy storage systems, and generators. The outputs of this tool are size requirements of all resources, as well as dispatch profile by resource, fuel requirements, and emissions. The size requirements are expressed as a pairing of power and energy capacities necessary to meet the balancing requirements. As part of the analytics suite, a LCC optimizer was developed that compares different hybrid energy storage system options based on a LCC to seek the lowest cost technology option.

\subsection{Approach and Data Used to Determine Balancing Requirements}

The fundamental approach of the PNNL methodology is outlined below. A full description of the methodology can be found in Makarov et al. (2008a). The approach uses historic load data and understanding of how the load forecasting errors are statistically distributed. In addition, wind profile data are necessary both from existing wind farms and new hypothetical wind resources that are presumed to be developed in the foreseeable future (Jacobson et al. 2004; Colella et al. 2004). The analytical approach includes the following components and individual steps:

1. Define a plausible wind capacity scenario by region. A $20 \%$ nation-wide RPS scenario for 2020 was selected, that was met primarily with new wind capacity. States with more aggressive RPS legislatures (i.e., California) were honored.

2. Placement of resources: Place hypothetical wind farms at plausible wind sites that are either at various stages in the permitting process or, alternatively, selected by the analyst based on resource potential and judgment.

3. Apply the statistics of wind and load forecasting errors from CAISO. Given PNNL's work with the CAISO, the experiences were utilized and projected to the entire WECC. It can be safely assumed that the experiences regarding load and wind forecasting errors are fairly uniformly applicable 
throughout the WECC region and by extension, throughout the United States. There is no reason to assume that specific regions have less or better capabilities to estimate the load and renewable energy forecasting error.

4. In addition, NREL wind datasets of hypothetical wind sites were utilized. Develop a stochastic process that generates a minute-by-minute balancing requirement for every sub-region with the 2020 wind capacity and load projections. The analysis assumes a consolidation of the balancing authorities into 22 sub-regions (see Figure 2.1). The output of this process will be the total balancing requirements applicable for the 2020 load and assumed total renewable energy capacity.

5. Define a set of technology options that will meet the total balancing requirements.

6. Analyze the LCC for technology options over a 50-year time horizon.

\subsubsection{Wind Datasets}

The NREL Wind Integration Datasets (NREL 2009) were utilized to determine the actual production of all wind sites in every NERC region. NREL datasets provided 10-minute interval production schedules for over 30,000 hypothetical wind sites. Wind production data are based on meso-scale wind simulation assuming the Vestas V-90 3MW wind turbine and a hub height of 100 meters above ground. Electricity production data are available for 1 year. Existing and additional (hypothetical) wind capacities are shown in Figure 5.1.

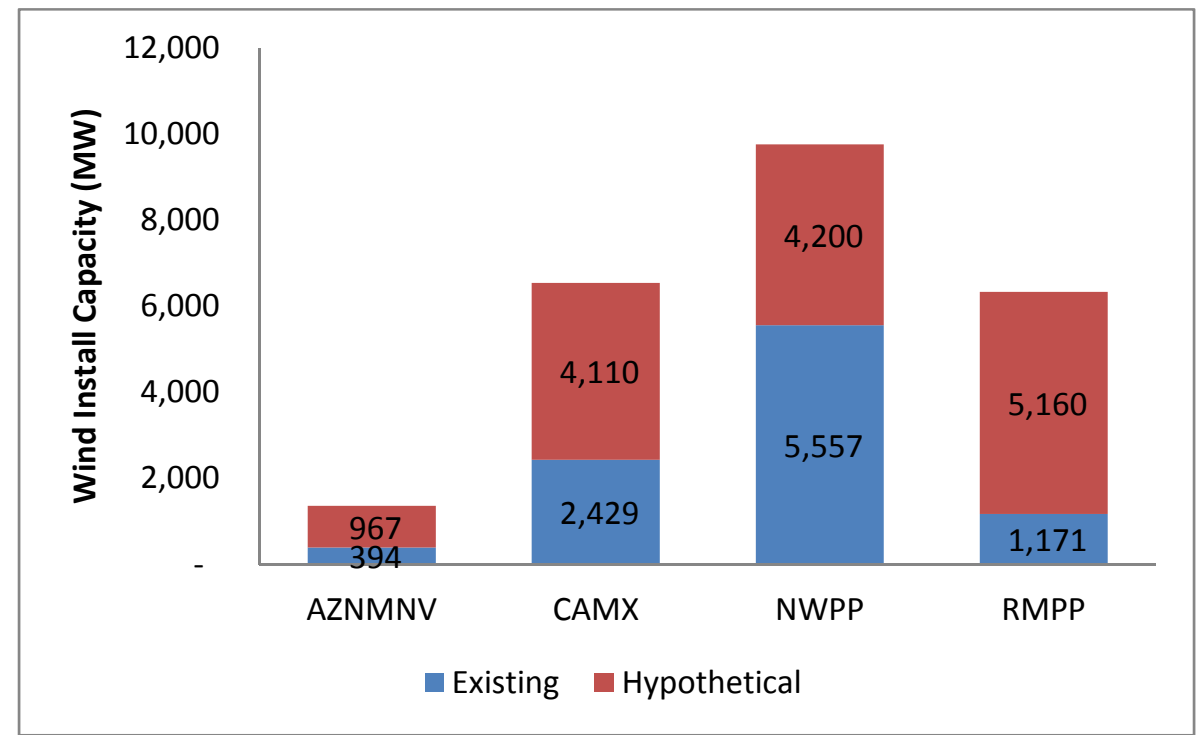

Figure 5.1. Wind Projection 2011-2020 for NERC regions in WECC

The Wind Integration Datasets from NREL are wind production simulated for 32,043 wind sites in the WECC system with 10-minute intervals. The information of the datasets is briefly shown in Table 5.1. The western wind datasets were produced by 3Tier Company using the Weather Research and Forecasting (WRF) mesoscale model. The modeled data were temporally sampled every 10 minutes and spatially sampled every arc-minute (around 2 kilometers). ${ }^{1}$

\footnotetext{
${ }^{1}$ http://www.nrel.gov/wind/integrationdatasets/western/methodology.html
} 
Table 5.1. Information About NREL Wind Integration Datasets

\begin{tabular}{ll}
\hline \multicolumn{1}{c}{ Produced By } & \multicolumn{1}{c}{ Western Dataset } \\
\hline & 3Tier Company \\
Mesoscale Model & WRF \\
Number of Output Points & 32,043 \\
Size of Output Point & 1 arc-minute ${ }^{(a)}$ \\
Output Point Capacity (MW) & 30 \\
Model Output Heights (m agl) & $100 \mathrm{~m}$ \\
Turbine Power Curves & Vestas V-90 3MW \\
\hline (a) One arc-minute of latitude is 1.825 km at any meridian. \\
One arc-minute of longitude is exactly 1.852 km at the \\
equator.
\end{tabular}

The placement of the new wind capacity is done by considering several factors, including information from grid operators about planned wind sites that are at various permitting stages, and judgment considering, best wind resources, and proximity to load or transmission lines. Based on NREL wind datasets, even when selecting only the best wind class ( 6 and 7) land areas in proximity to transmission above $230 \mathrm{kV}$, the suitable hypothetical wind farm sites and total capacity is significantly larger than what is needed for the generation capacity additions. Figure 5.2 shows the selected wind capacity distribution by state. The average capacity factor of the new wind sites is around 35\%. Figure 5.3 illustrates all additional and currently existing wind sites in the WECC.

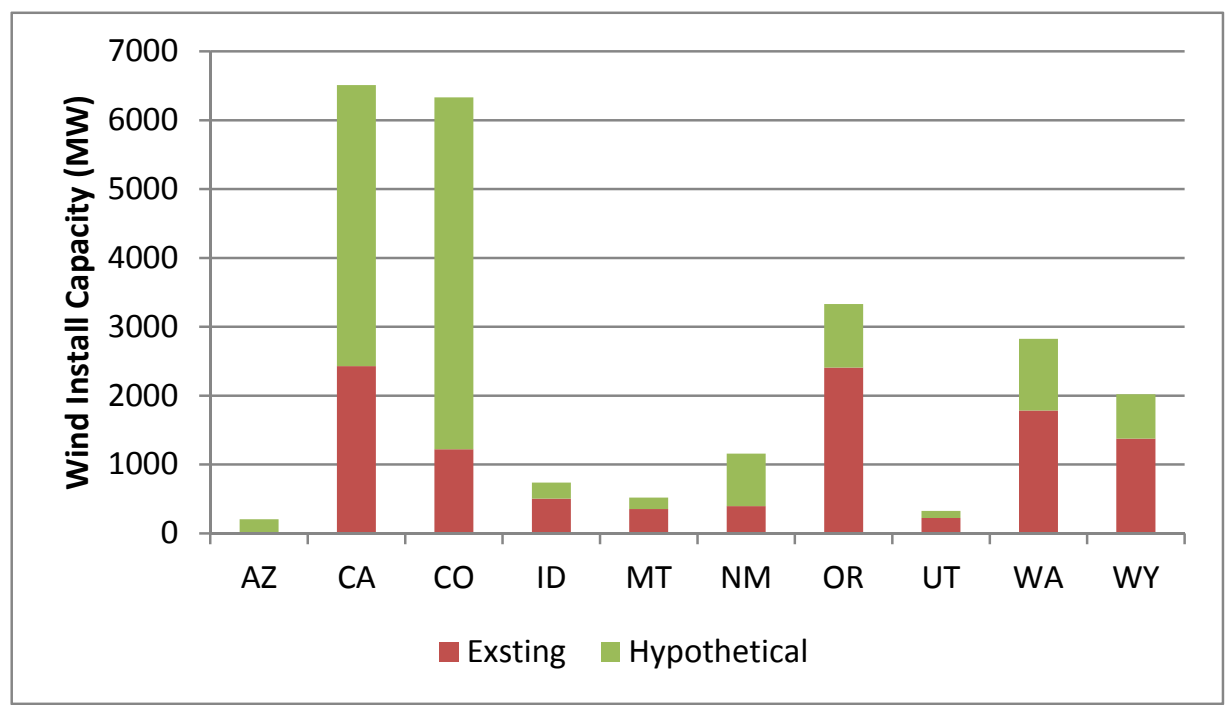

Figure 5.2. Distribution of Wind Capacity by States 


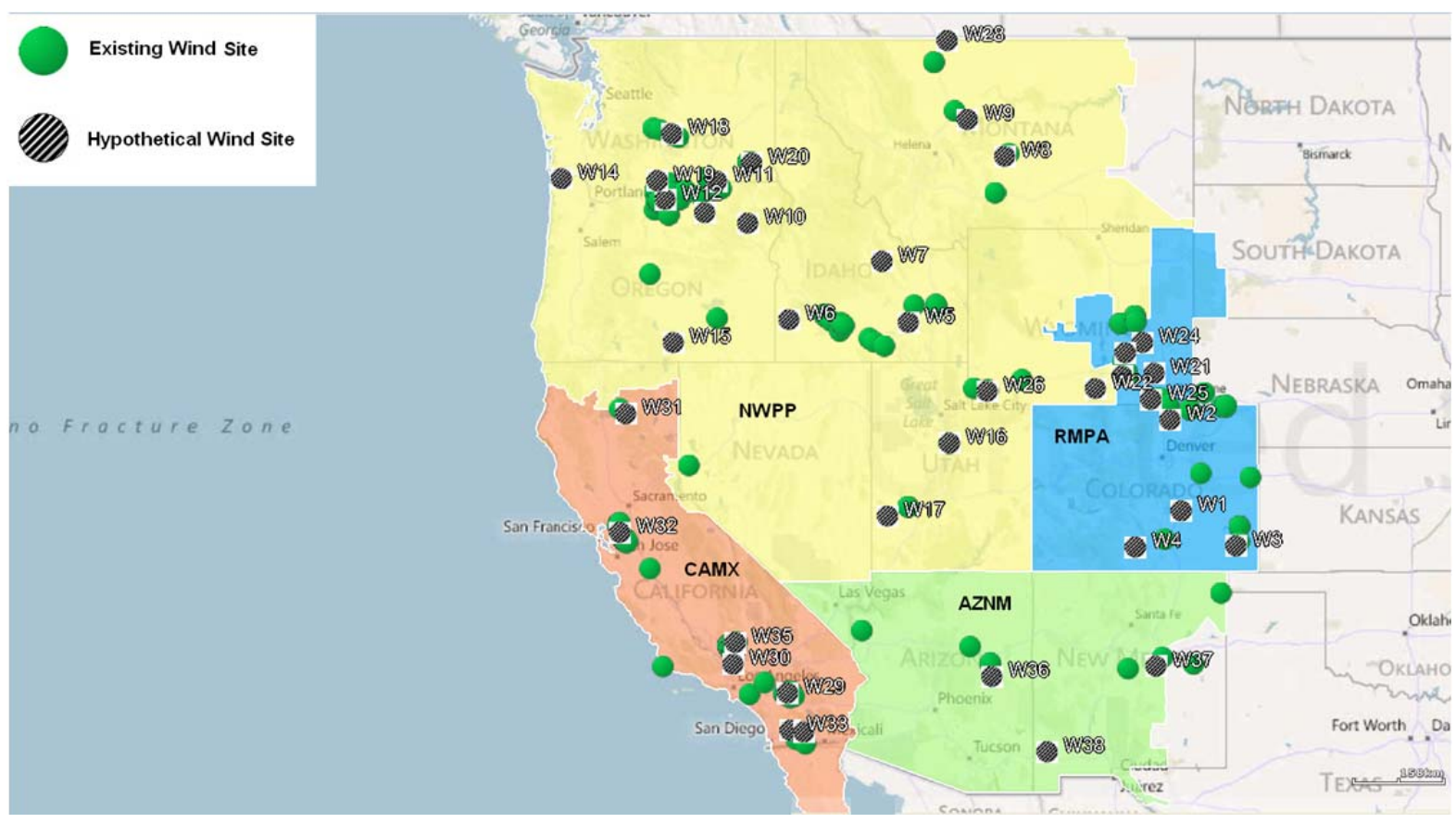

Figure 5.3. Location of Existing and Arbitrarily Sited Future Wind Plants in WECC

To obtain the balancing requirements, minute-by-minute wind production data are needed. However, NREL wind datasets are created with 10-minute interval. Therefore, the 10-minute interval data are interpolated to generate the required minute-by-minute data by using the interpolation method provided by 3Tier Company. The hourly wind forecast is obtained by averaging wind production of every hour and superimposing wind forecast error on the hourly average. Wind power generation in 2020 for each wind plant is assumed to be the same as that of the year 2006. The wind forecast error is generated by using a multivariate stationary Markov Chain to reproduce the statistical characteristics including the standard deviation, mean value, and autocorrelation of current wind forecast error (Makarov 2010b). The statistical information of hour-ahead wind forecast error is shown in Table 5.2.

\subsubsection{Load Datasets}

The minute-by-minute actual load data of 2009 and hour-ahead load forecast of 2020 for every balancing authority are obtained from WECC. The within hour variations of the 2009 load are added to the 2020 hourly load to get minute-by-minute load data for the year 2020. Then, the loads are aggregated to obtain the minute-by-minute load data for every NERC region. In this analysis, we assume the hourly generation schedule is the same as the hour-ahead load forecast. Load growth assumptions are inherited from the Transmission Expansion Planning and Policy Committee (TEPPC) 2020 15\% renewable case.

The hourly load forecast is obtained by adding load forecast error to the hourly average of load. The load forecast error is generated by using a multivariate stationary Markov Chain to reproduce the statistical characteristics including the standard deviation, mean value, and autocorrelation of current load forecast error. Table 5.2 and Table 5.3 shows the statistics for the load forecast errors, respectively. 
Table 5.2. Statistics of Hour-Ahead Wind Forecast Error (the percentage values are based on installed wind capacity)

\begin{tabular}{lllll}
\hline & AZNM & CAMX & NWPP & RMPA \\
\hline Mean (\%) & -0.03 & -0.68 & -0.56 & -0.05 \\
Standard Deviation (\%) & 7 & 7 & 7 & 7 \\
Auto Correlation & 0.8887 & 0.9386 & 0.9388 & 0.9143 \\
\hline
\end{tabular}

Table 5.3. Statistics of Hour-Ahead Load Forecast Error (the percentage values are based on peak load)

\begin{tabular}{lllll}
\hline & AZNM & CAMX & NWPP & RMPA \\
\hline Mean (\%) & -0.61 & 0.42 & 0.14 & 1.93 \\
Standard Deviation (\%) & 1.99 & 1.17 & 1.04 & 2.83 \\
Auto Correlation & 0.9559 & 0.9282 & 0.937 & 0.9255 \\
\hline
\end{tabular}

\subsubsection{Balancing Service Requirement}

The power system control objective is to minimize its ACE to the extent that complies with NERC Control Performance Standards. Therefore, the "ideal" regulation/load following signal is the signal that minimizes deviations of ACE from zero when they exceed a certain thresholds:

$$
\begin{aligned}
& -A C E=-\left(I_{a}-I_{s}\right)+\underbrace{10 B\left(F_{a}-F_{s}\right)}_{\text {Neglected }} \\
& \approx G_{a}-L_{a} \rightarrow \min
\end{aligned}
$$

where

$$
\begin{aligned}
I & =\text { interchange } \\
F & =\text { frequency } \\
a \text { subscript } & =\text { actual } \\
S & =\text { schedule } \\
G_{a} & =\text { actual generation } \\
L_{a} & =\text { actual load within the control area. }
\end{aligned}
$$

Extending the generation component in the ACE equation,

$$
G_{a}=G_{s}+G_{I B}
$$

where actual generation, $G_{a}$, is obtained where the subscript $\mathrm{s}$ is hour-ahead schedule, and $I B$ is the generation required to meet intra-hour balancing requirement. The generator output is assumed to not deviate from its schedule. That is,

$$
G_{s}=L_{f_{-} h a}
$$

where $f_{-} h a$ denotes hour-ahead forecast. 
In Equation (5.1), set $A C E$ to zero, the intra-hour balancing signal $G_{I B}$ can be calculated by equation below.

$$
G_{I B}=L_{a}-L_{f_{-} h a}
$$

When wind generation is included, wind is counted as negative load. Therefore,

$$
G_{I B}=\left(L_{a}-L_{f_{-} h a}\right)-\left(G_{a}^{w}-G_{f_{-} h a}^{w}\right)
$$

The first part of the equation above $\left(L_{a}-L_{f_{-} h a}\right)$ is also called the balancing requirements caused by load uncertainty, and the second part $\left(G_{a}^{w}-G_{f_{-} h a}^{w}\right)$ is also called the balancing requirements caused by wind uncertainty.

The terms in Equation (5.5), $L_{f_{-} h a}$ and $G_{f_{-} h a}^{w}$, are then generated using a stationary multivariate Markov Chain, that meets all of the statistics including the standard deviation, mean, and autocorrelation of current wind and load forecasting errors.

Figure 5.4 illustrates the concept of over- and under-generation as a result of the forecasting errors for both the load and the wind energy production. The over- and under-generation is then the balancing signal, which balances generation and load and minimizes the ACE in each of the four sub-regions in the western interconnection. Hence, a positive balancing signal represents over-generation, and vice versa.

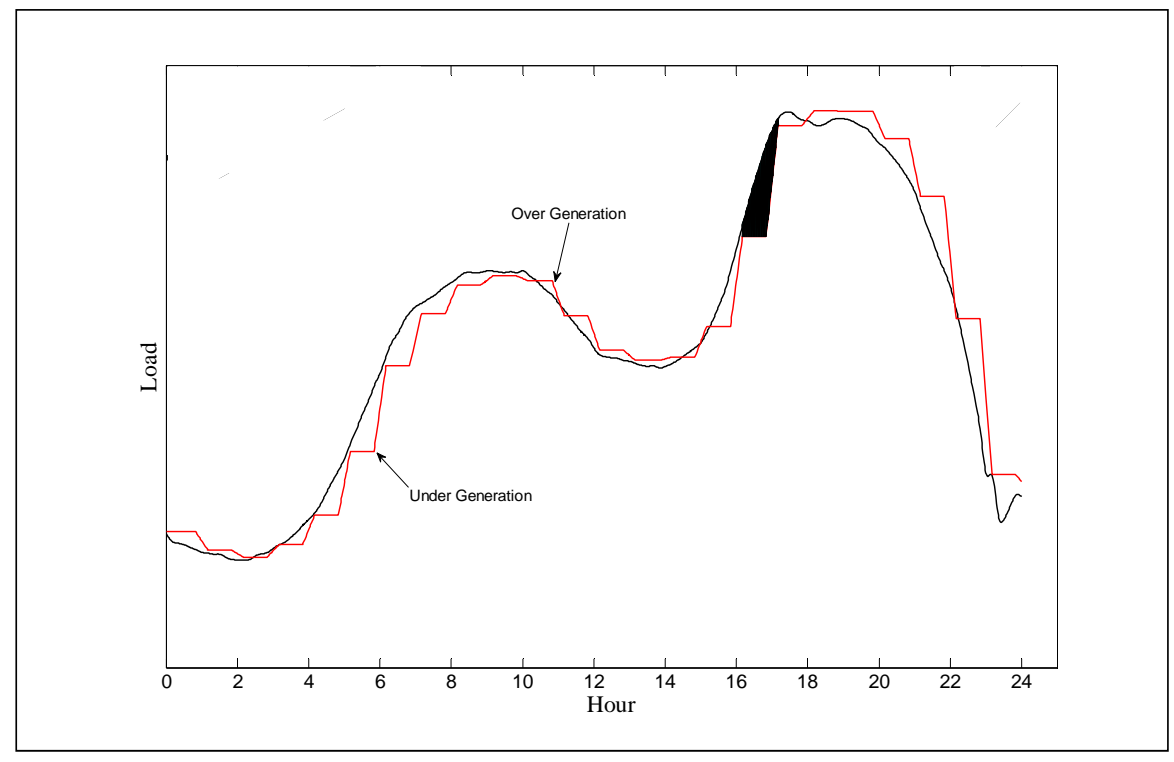

Figure 5.4. Illustration of Intra-Hour Balancing Signal

\subsubsection{Consolidation of Balancing Areas}

To simplify the analysis, balancing authorities (BA) in the WECC are assumed to be consolidated into four NERC sub-regions. This simplification reduces the analysis complexity significantly. Instead of performing a BA-by-BA analysis for the 32 BAs and combining the results for the WECC, the 
consolidation collapsed the complexity into four zones. There are implications to this simplification. The consolidation of BA will provide greater sharing of balancing and reserve resources among all constituents and offer opportunities to more effectively utilize the higher degrees of diversity of the variable renewable energy resources across the entire WECC. As a consequence, the total balancing requirements of WECC in this assessment are likely to be underestimated. This, in turn, will lead to an underestimation of the future resource requirements under the existing BA regime.

\subsubsection{Resulting Total and Additional Balancing Signals}

The total balancing requirements for each sub-region are estimated utilizing the wind and load datasets as discussed above. In addition, the balancing requirements caused by incremental demand and hypothetical wind capacity are also estimated. Figure 5.5 and Figure 5.6 illustrate an example of the resulting balancing requirements signal of a NERC region for the whole month and one typical day, respectively. These estimated values represent the total requirements, as opposed to additional requirements. These figures are based on BPA's customary $99.5 \%$ probability bound that meets $99.5 \%$ of all balancing requirements. That means that $0.5 \%$ of all of the anticipated balancing capacity exceeds that bound. For a $100 \%$ probability bound, the maximum balancing requirements are likely to increase.

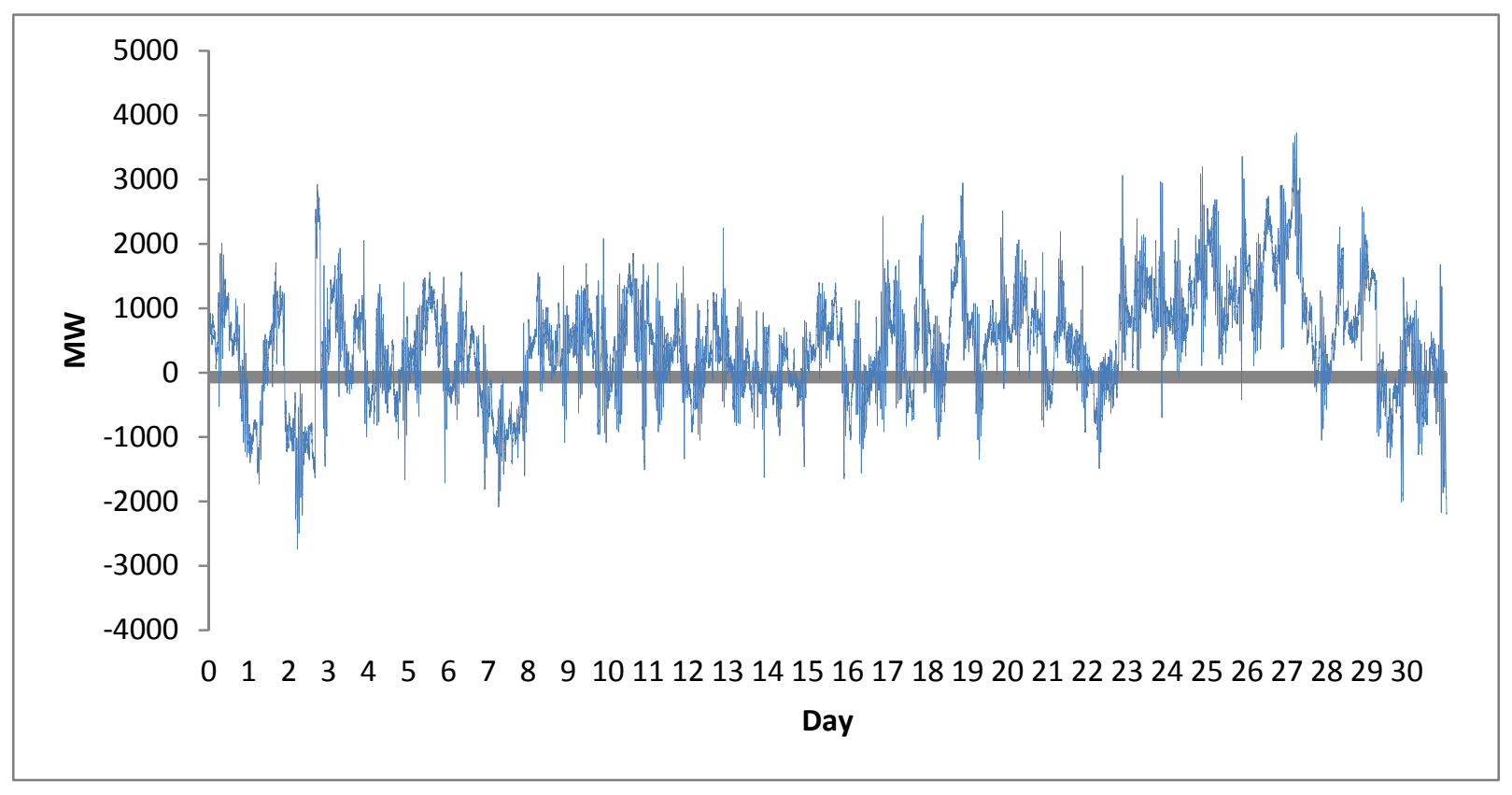

Figure 5.5. An Example of Total Balancing Requirements for the Month of August 2020 


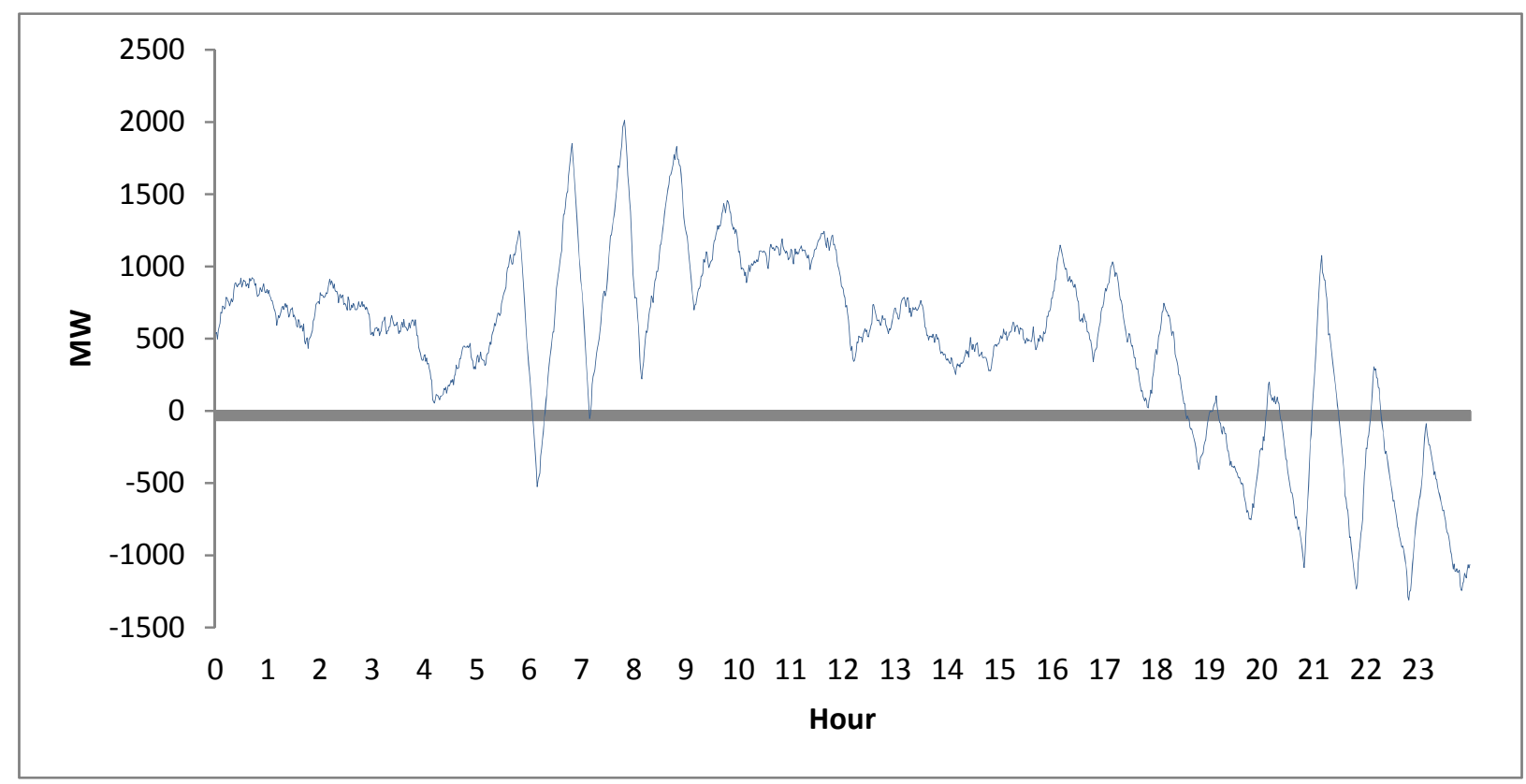

Figure 5.6. An Example of Total Balancing Requirements for One Typical Day in August 2020

The balancing signal shown in Figure 5.6 exhibits a spectrum of cycling or oscillatory content. Cycles at lower frequencies with periods of several hours (inter-hour) are less challenging to be managed. They can be accommodated in real-time energy markets (for competitive wholesale markets) or in a redispatch process when the generation schedule deviates too much from the load conditions. Balancing cycles of lower frequency are not considered in this study. Cycles within the hour (intra-hour balancing) are the key focus of this analysis. They are more challenging to provide because of their high ramping rates, which require grid assets that have a high degree of flexibility to be ramped up and down within short period of time. The rest of this section discusses the filtering strategies to extract the intra-hour cycling from the original balancing signal. The value of deploying energy storage for energy arbitrage is also investigated in this study and presented in Section 7 of this report.

\subsubsection{Spectral Analysis and Extraction of Intra-Hour Balancing Signal}

A high-pass filter was designed to filter out the fast cycles (intra-hour and real-time components) from the original balancing signal (Makarov 2010a). The cut-off frequencies for the filter were $f_{l}=1.157 \mathrm{e}-5 \mathrm{~Hz}$ and $f_{u}=0.2 \mathrm{~Hz}$. The spectral analysis of the balancing signal illustrates the oscillatory content in the signal. The results of the spectral analysis are shown conceptually in Figure 5.7 and Figure 5.8. Table 5.4 displays the frequency limits for the high-pass filter design. 


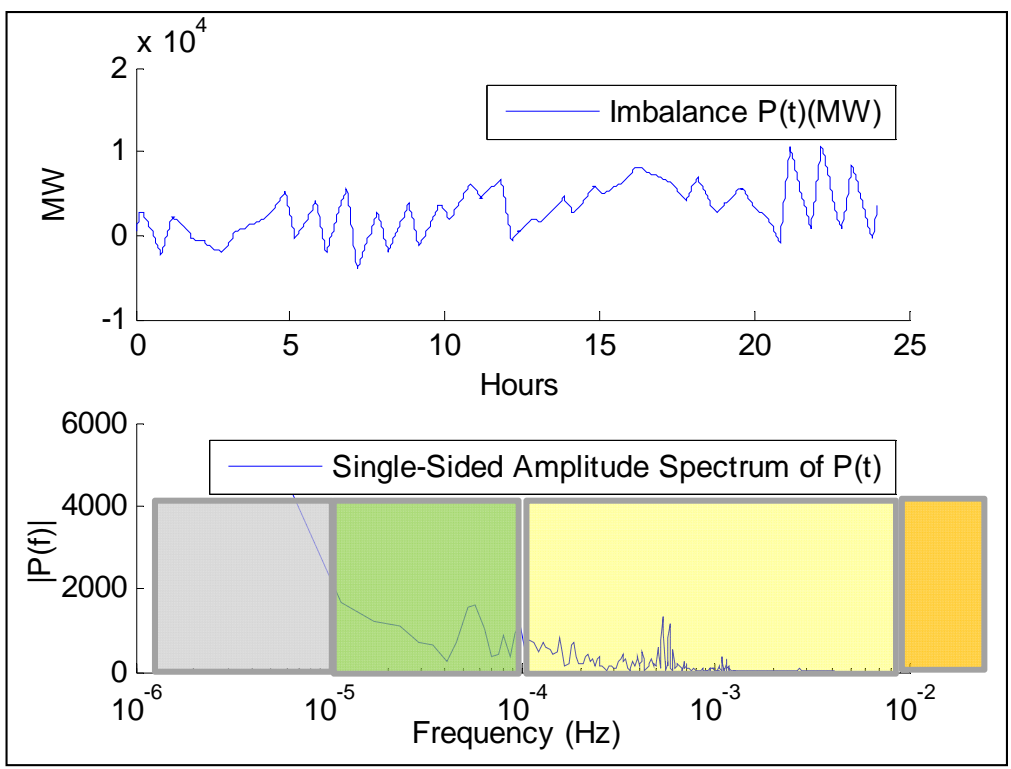

Figure 5.7. Spectral Analysis of Balancing Signal

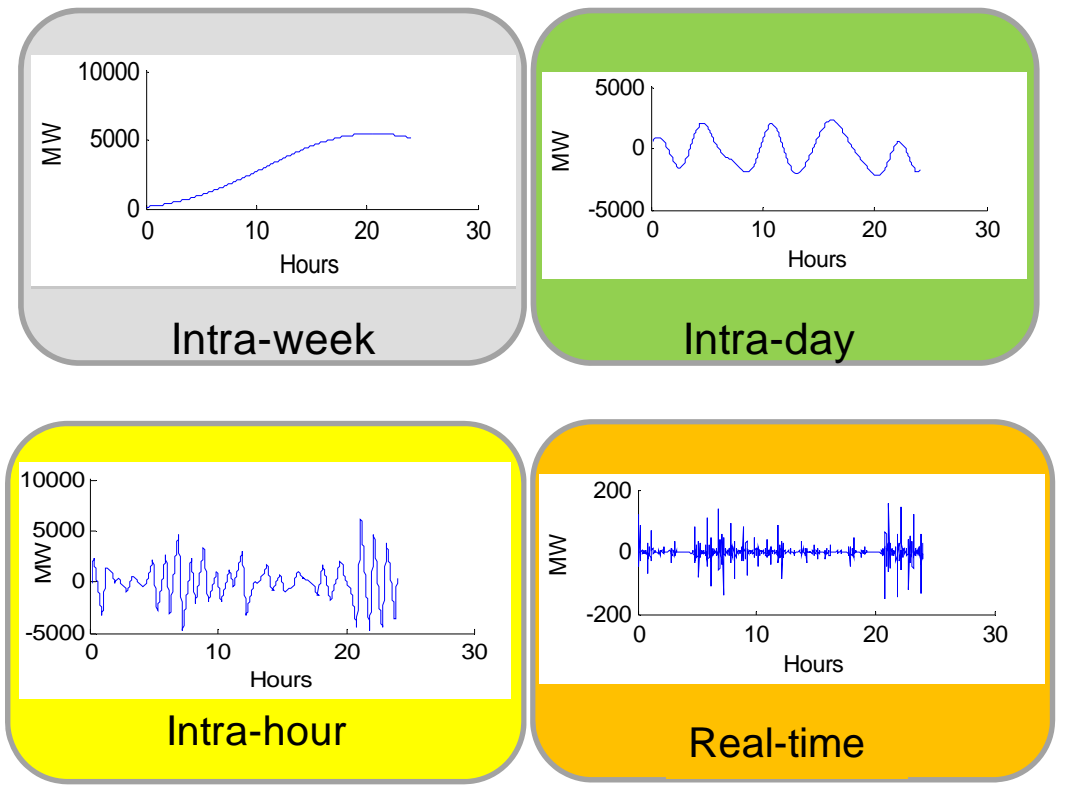

Figure 5.8. Components of Decomposed Balancing Signal

Table 5.4. Frequency Limits of Components of the Balancing Signal

\begin{tabular}{lccccc}
\hline No. & Component & $f_{l}(\mathrm{~Hz})$ & $f_{u}(\mathrm{~Hz})$ & Period of $f_{l}$ & Period of $f_{u}$ \\
\hline 1 & Intra-week & 0 & $1.157 \mathrm{e}-05$ & Inf & 24 hours \\
2 & Intra-day & $1.157 \mathrm{e}-05$ & $1.388 \mathrm{e}-04$ & 24 hours & 2 hours \\
3 & Intra-hour & $1.388 \mathrm{e}-04$ & 0.0083 & 2 hours & 2 minutes \\
4 & Real-time & 0.0083 & 0.2 & 2 minutes & 5 seconds \\
\hline
\end{tabular}




\subsection{Sizing Storage to Meet Balancing Requirements}

Sizing energy storage equipment requires determining and selecting two capacity parameters: the power rating (MW) to meet a load or power target, and an energy rating (MWh) that is expected to be delivered to the grid or absorbed by the grid at any given cycle. Because generators are not as energy limited as storage are the energy rating or energy capacity is not a design criterion (it is assumed there is an unlimited supply of natural gas, coal, uranium). However, for storage resources and to a certain degree demand resources as well, the energy capacity is a very important selection and design criterion and determines the control strategy for a storage device.

To estimate the power and energy capacity for storage technologies to meet the balancing requirements an engineering model was applied that determined the minimal size requirements in terms of MW and MWh, to meet both maximum power requirements and the electric energy necessary to balancing requirements as shown in Figure 5.5. The outcomes for the sizing analysis are a pair of power and energy capacities or ratings for each technology.

\subsubsection{Sizing Hybrid Technology Options for Balancing Services}

To determine power and energy requirements for two storage technologies, the intra-hour balancing signal, is divided into two components: a "slow storage" and a "fast storage" component. These balancing components are satisfied by two storage technologies with different technical and economic characteristics. In this study, 12 combinations of "slow storage" and "fast storage" components are defined, including the extreme cases of one single technology. To determine the optimal combination, the 12 technology shares are further optimized using the economic procedure discussed in Section 5.

The lower frequency contents of the intra-hour balancing signal are assigned to the "slow storage" component, while the higher frequency contents of the intra-hour balancing signal are assigned to the other component ("fast storage"). The "slow storage" component is satisfied by a storage technology with limitations in ramp rate caused by technical capabilities and/or wear and tear. An example of "slow storage" technology is CAES with a ramp rate limitation of $30 \%$ rated power per minute. The "fast storage" component is satisfied by a storage technology with a very high ramp rate and cycling capabilities such as flywheels (with a ramp rate of more than 100\% rated power per minute).

The methodology to assign the portions of the intra-hour balancing signal is as follows. In the frequency domain, a cut frequency $f c$ is defined; where $f_{c}$ marks the limit between the slow storage component and the fast storage component. The frequency contents of the balancing signal larger than $f_{c}$ belong to the fast storage component while the frequency contents lower than $f c$ belongs to the slow storage component. Technology share options are defined by choosing 12 different values of fc along the frequency spectrum of the intra-hour balancing signal. When $f_{c}$ equals and arbitrary frequency $f 2$

$\left(f_{c}=f 2\right)$, all the balancing is provided by the fast storage. Contrary, when the cut frequency $f_{c}$ is smallest $f c=(1 /(2 * 60 * 60)$, all the balancing is provided with the slow storage technology. Figure 5.9 illustrates this procedure. 


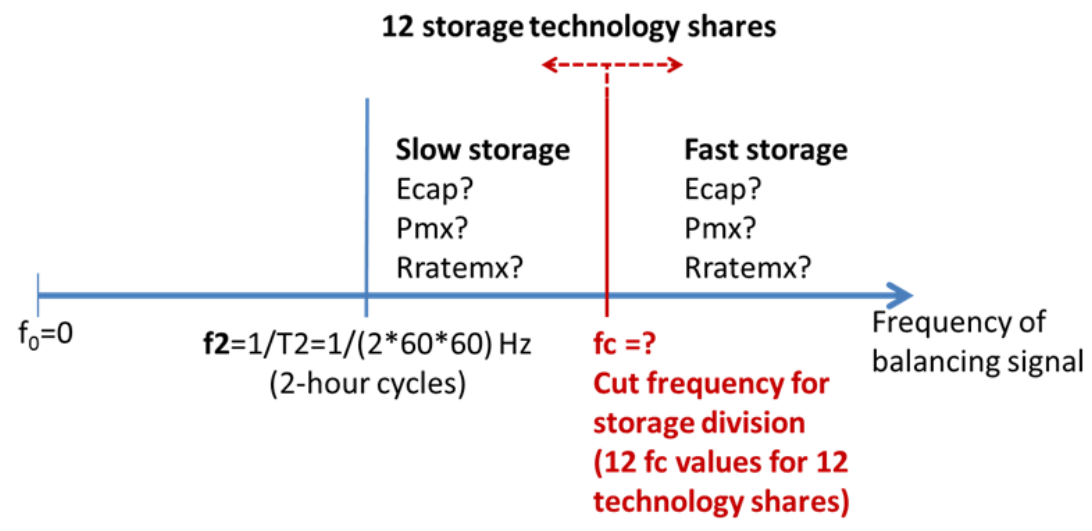

Figure 5.9. Division of Balancing Signal for Two Storage Technologies

Each value of $f_{c}$ defines a pairing of slow and fast storage sizes, together adding up to the total storage size. The sum of all technology pairings is always the same. The storage size of the two technologies is described by the energy requirement $(\mathrm{kWh})$ and power requirement $(\mathrm{kW})$. Figures 5.10 and 5.11 display the storage sizes in terms of energy requirement $(\mathrm{kWh})$ and power requirement $(\mathrm{kW})$ for the two storage technologies as a function of $f c$, going from $f_{2}$ (2-hour cycles) to the maximum frequency (half the sampling frequency $(1 / 60 \mathrm{~Hz})$ ). Figure 5.12 show the ramp rates that each storage technology faces as a function of $f c$. The ramp rate is checked against the ramp rate limitations of each technology. No ramp rate constraints were binding in the cases studied.

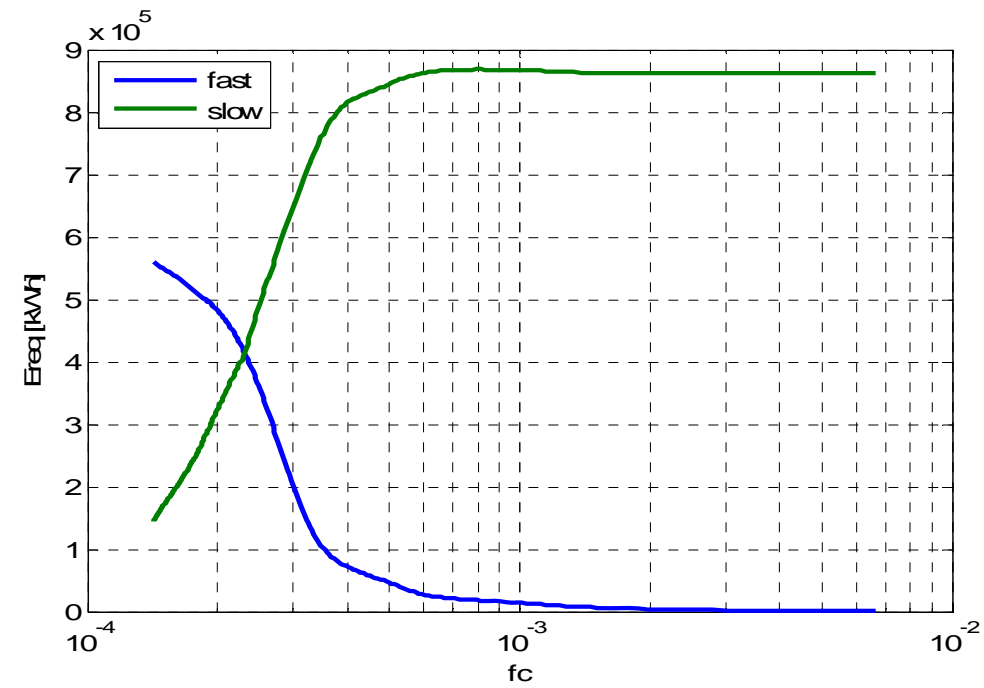

Figure 5.10. Storage Sizes in Terms of Energy Requirement (kWh) for the Two Storage Technologies 


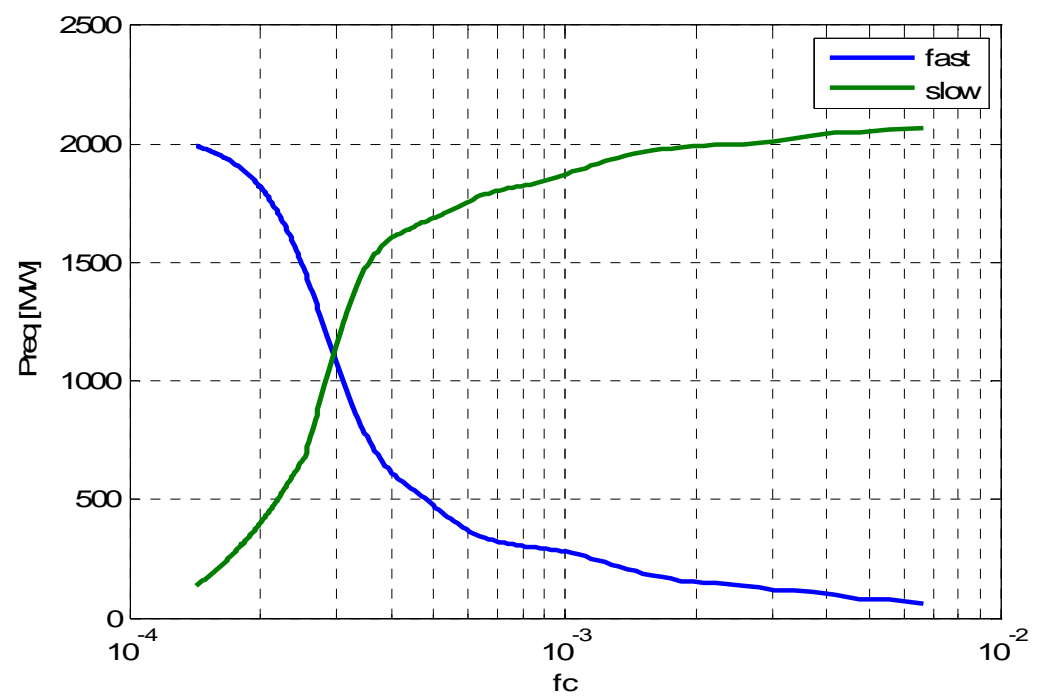

Figure 5.11. Storage Sizes in Terms of Power Requirement $(\mathrm{kW})$ for the Two Storage Technologies

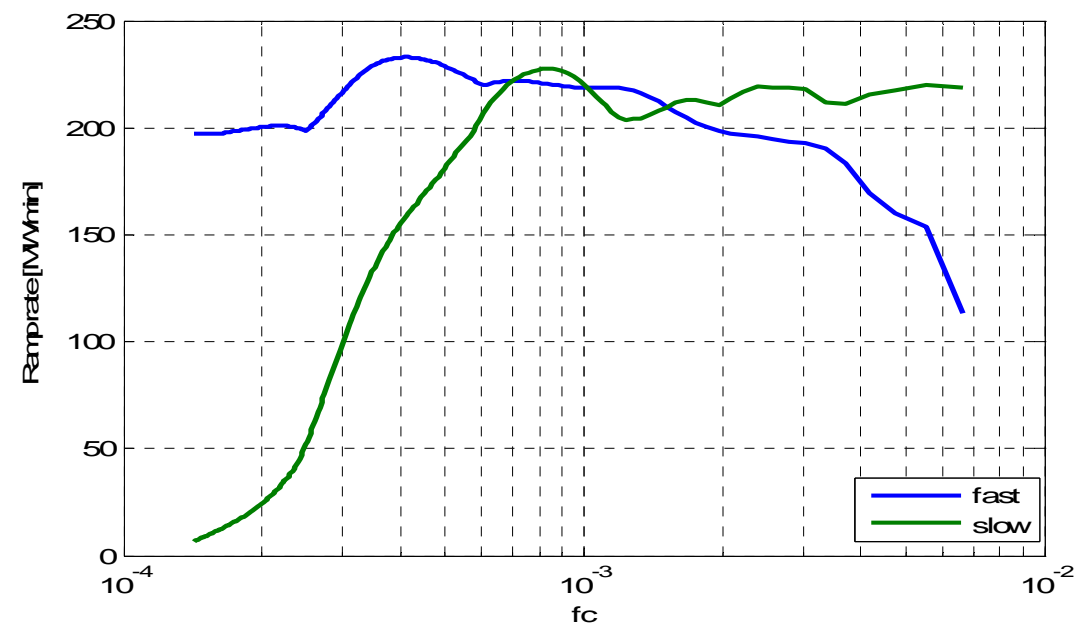

Figure 5.12. Storage Sizes in Terms of Maximum Ramp Rate Requirement (MW/min) for the Two Storage Technologies

The optimal combination of fast storage and slow storage technologies is determined based on total LCC analyses as discussed in Section 5. For each technology share, the battery capacity and DOD is varied from $0 \%$ to $100 \%$ and the least cost alternative is selected. The least cost alternative for each technology share is, in turn, identified and compared against the least cost alternative for every other technology share in order to determine the most cost-effective technology share for each case.

As shown in Figures 5.10 and 5.11, in each case, the technology shares are designed with heavy reliance on one technology at one end of the spectrum and heavy reliance on the alternative technology at the other end. For example, under Technology Share 1 in a case including Li-ion and DR, the power demand for DR is $470 \mathrm{MW}$ (about 5\% of DR needed to provide all the balancing) while the power demand for Li-ion is 2,340 MW (about 95\% of Li-ion needed to provide all the balancing). Near the 
other end of the spectrum, Technology Share 10 assigns 8,210 MW of power demand (about 95\% of DR needed to provide all the balancing) to DR and $130 \mathrm{MW}$ (about 5\% of DR needed to provide all the balancing) of power demand to Li-ion.

The results for the DR + Li-ion, for CAMX, are presented in Figure 5.13 and Figure 5.14 (for 2020 cost and 2011 costs, respectively). In Figure 5.13, the least cost alternative (Technology Share 0) is the one with $100 \% \mathrm{Li}$-ion (and 0\% DR). This outcome is driven by the relatively lower capital costs associated with the Li-ion technology considering the 2020 cost assumptions (that is, $100 \%$ Li-ion (Technology Share 0) is less expensive than 100\% DR (Technology Share 11), see Figure 5.13). The cost curve is upward sloping. However, the results change for the 2011 cost assumptions; a combination of Li-ion and DR (Technology Share 6) is more cost-effective. The cost curve has a more irregular shape (Figure 5.14). Notice that in Figure 5.14, that 100\% Li-ion (Technology Share 0) is now more expensive than $100 \%$ DR (Technology Share 11). This is a clear contrast from Figure 5.13 only due to the change in cost assumptions. Additionally, notice that a non-linearity emerges in Figure 5.14; this non-linearity is due to the technical sizing and operating assumptions of DR and economic costs model. On the technical side, DR availability from EVs (see Figures B.5 and B.6 in Appendix B) is different from the availability of stationary storage; DR resource availability changes throughout the day according to driving and charging patterns of EVs. On the economic side, DR has capital costs that depends only on power capacity, while stationary storage capital costs depend on both energy and power capacity. The nonlinearities of Figure 5.14 are still present in the case of Figure 5.13, however, these non-linearities are masked due to the 2020 cost assumptions.

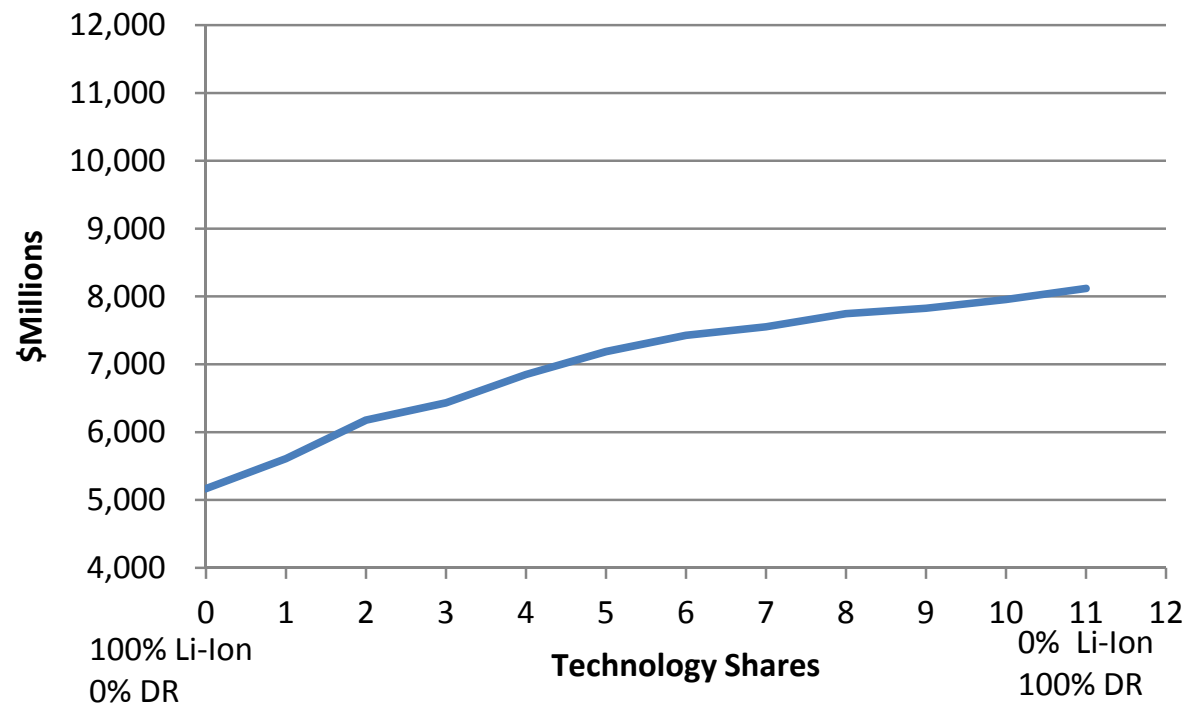

Figure 5.13. Total 50-Year LCCs for Li-ion +DR Technology Shares for 2020 Cost Assumptions. "Winner takes all” situation present in most cases studied. 


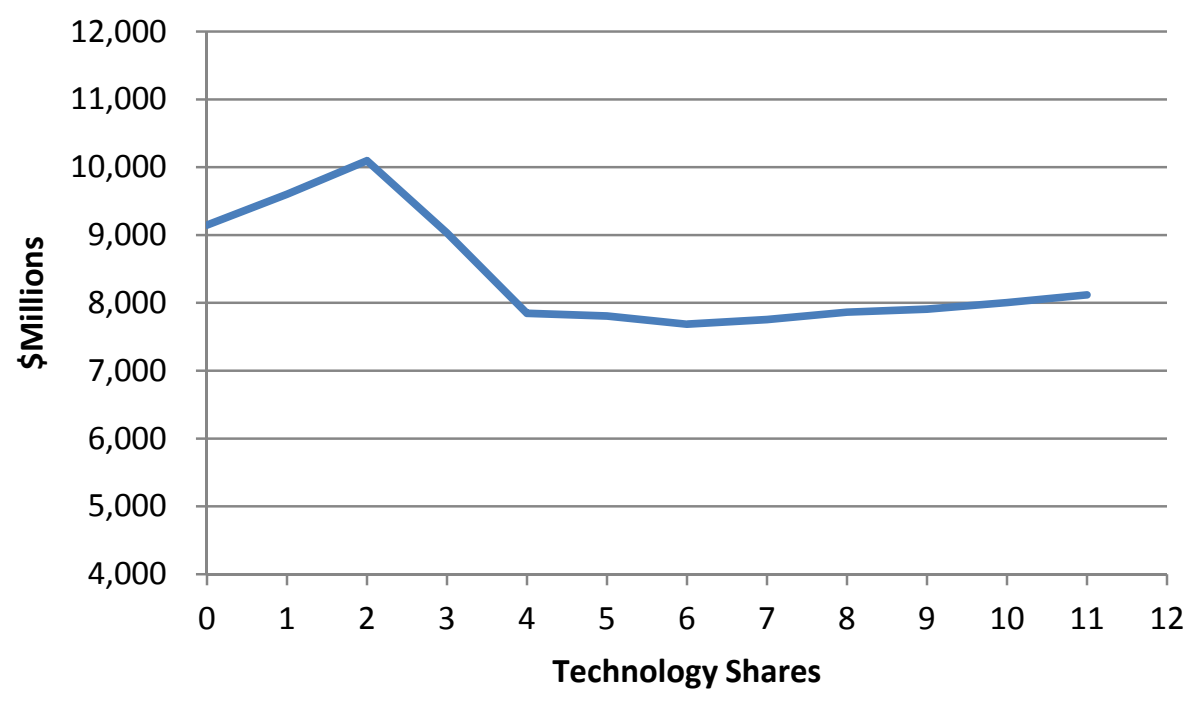

Figure 5.14. Total 50-Year LCCs for Li-ion +DR Technology Shares for 2011 Cost Assumptions. Optimal combination (technology share 6) only present in two cases under 2011 cost assumptions.

There are two possible reasons for the sizing of hybrid energy storage to result in a combination of technologies (as in Figure 5.14) instead of a "winner takes all” situation (as in Figure 5.13): a) binding of technical constraints such as ramp rate and b) nonlinearities in the objective function due to technical operating strategies and cost model. Technical constraints (such as ramp rate limitations) were nonbinding, meaning that ramp rate characteristics was not a differentiating feature of any of the technologies analyzed. This indicated that the balancing requirements represented by minute-to-minute changes could be met by all technologies.

For two hybrid technology pairings, the optimization indicated a non-trivial solution in which winner did not take all shares. As can be seen in Figure 5.14, the optimum suggest a 60/40 sharing between Li-ion and DR technologies for the 2011 cost estimates. This solution was primarily attributable to the non-constant DR resource availability (EV charging occurs primarily at night and after the morning commute) and the cost differential between DR and Li-ion.

The other technology pairing with a non-trivial solution was $\mathrm{PH}$ with multiple mode changes and flywheels. The underlying cause for the optimum we very similar to the $\mathrm{DR}$ and Li-ion case. It was primarily driven by the unavailability of the hydro resource during the mode switching and the relative cost between $\mathrm{PH}$ and a flywheel.

In addition to the case comprised of Li-ion and DR, the research team also examined the following cases: Na-S + DR, CAES + flywheels, PH with multiple mode changes + Na-S, PH with two daily mode changes + Na-S, PH with multiple mode changes + flywheels, and PH with two daily mode changes + flywheels. Each case was also examined using 2011 cost assumptions and was run through each sensitivity analysis, as described in Section 5.5. The primary conclusions drawn from this analysis are as follows. 
Under the 2020 price scenario and all sensitivity analyses described in Section 5.5 (with the exception of 2011 price scenario), a "winner takes all” condition is present where the technology share comprised primarily of the least cost technology is always the most cost-effective hybrid solution. This condition also holds true for each case under the 2011 price scenario with the exception of combinations of Li-ion and DR technologies, and combinations of pumped hydro with multiple mode change and flywheels. This result stems from the non-linearity in these two combination cases. For the Li-ion and DR combination case under the 2011 price scenario, the least cost technology share was $60 \%$ DR and $40 \%$ Li-ion in most regions. There was a non-linearity originated by the availability of DR. For the pumped hydro with multiple mode changes plus flywheels combination case, the least cost technology share was $60 \% \mathrm{PH}$ and 40\% flywheel for the CAMX area and under the 2011 price scenario. The non-linearity in this case is stemmed from the waiting period between mode changes of pumped hydro. The non-linearity influences the technology share outcome when the costs of the two technologies are comparable. 



\subsection{Economic Analysis Methodology and Results}

\subsection{Cost Analysis Framework}

That energy storage can enable higher levels of wind and solar energy integration is a necessary but not singular condition for market acceptance. There are competing technologies (e.g., CT and DR) that could be used similarly to address technical issues resulting from the variable nature of wind and solar energy.

To examine the competitiveness for energy storage, the analytical framework for Doane et al. (1976) was adapted and the parameters outlined later in this report were used to conduct an economic assessment of the following nine alternative technology cases:

1. CT

2. Na-S batteries plus CC plants

3. Li-ion batteries plus CC plants

4. Flywheels plus CC plants

5. CAES plus CC plants

6. Redox (reduction-oxidation) flow batteries plus CC plants

7. PH with multiple mode changes per day plus CC plants

8. $\quad$ PH with 2 mode changes per day plus CC plants

9. DR.

The cost model used to support this analysis examined all initial and recurrent costs, property and income taxes, depreciation, borrowing costs, and insurance premiums. Major cost assumptions are presented in Table 6.1. The cost model presents results in 2011 dollars, and treats interest and inflation in a systematic manner and distinguishes between costs that occur annually and those that occur in a single year. The cost model generated annualized cost and total LCC estimates for each case, as required to meet the balancing requirements for each of the four WECC sub-regions: the NWPP, RMPA, CaliforniaMexico Power Area (CAMX), and Arizona-New Mexico-Southern Nevada Power Area (AZNM). See Figure 2.1 for a geographic overview of each WECC sub-region.

\subsection{Optimizing the Battery Capacity}

An important factor in minimizing the costs associated with alternative energy storage cases is optimally sizing the battery capacity. In effect, one could size up the energy storage capacity to reduce the DOD and increase the economic life of the battery systems, as demonstrated in the calculations performed by the research team in Table 6.2. Increasing the battery capacity drives up the initial capital costs but reduces the DOD requirements and extends the life cycle of batteries, thus reducing interim capital costs. To account for natural aging the maximum life cycles for Na-S, 
Li-ion, and redox flow batteries were constrained to 13, 10, and 25 years, respectively. The maximum life cycle for flywheels was constrained to 25 years. Table 6.2 presents the data computed for Case 3 (Na-S plus CC).

Table 6.1. Utility Description Data and General Economic Parameters

\begin{tabular}{lr}
\hline Utility Description Data & \\
System Operating Lifetime & 50 years \\
Effective Income Tax Rate & $40 \%$ \\
After Tax Weighted Cost of Capital & $8 \%$ \\
Annual Other Taxes and Insurance Premiums as & \\
Fraction of Capital Investment & $2 \%$ \\
Base Year for Dollars & 2011 \\
General Economic Parameters & \\
Rate of General Inflation & $1.8 \%$ \\
Escalation Rate for Capital Costs & $1.8 \%$ \\
Escalation Rate for Operating and Maintenance & \\
Costs & $1.8 \%$ \\
Escalation Rate for Fuel Costs & $3.2 \%$ \\
\hline
\end{tabular}

Table 6.2. Relationship between DOD, Battery Capacity, and Life Cycle

\begin{tabular}{ccc}
\hline DOD & $\begin{array}{c}\text { Battery } \\
\text { Capacity(MWh) }\end{array}$ & $\begin{array}{c}\text { Life-Cycle } \\
\text { (Years) }\end{array}$ \\
\hline 0.05 & 11,748 & 240 \\
0.10 & 5,874 & 82 \\
0.15 & 3,906 & 43 \\
0.20 & 2,937 & 27 \\
0.25 & 2,350 & 18 \\
0.30 & 1,938 & 12 \\
0.40 & 1,468 & 7.5 \\
0.50 & 1,175 & 5.0 \\
0.75 & 781 & 2.7 \\
0.85 & 691 & 2.3 \\
0.95 & 618 & 2.0 \\
\hline
\end{tabular}

DOD, battery capacity, life cycle, and discount rates are the parameters used to establish the optimum battery size based on an assessment of the present value LCC for each DOD level, as demonstrated in Figure 6.1. In effect, upsizing the battery would increase initial capital costs but would extend the useful life of the technology, thus requiring fewer battery purchases over the 50-year analysis time horizon. While essential, the data in Table 6.2 are not sufficient to determine the optimum battery capacity required to minimize costs because you must build these values into the cost model to determine their impacts on costs. Though increasing the depth of discharge reduces the initial capital costs associated with investments in energy storage options, the life cycle falls significantly at higher DOD levels, thus 
requiring numerous interim capital investments in future years. Thus, a system that is upsized with a useful life of 10 years would result in relatively high capital costs but would require only five battery purchases over the 50-year analysis time horizon. On the other hand, a battery system with a 5-year life cycle would be smaller with lower initial capital costs but the service provider would need to purchase ten battery systems over the 50-year analysis time horizon. The cost model would calculate present value costs for each of these systems and the least cost approach would be compared against those chosen for the other cases. Tables relating DOD to battery capacity and life cycle were constructed for all battery and flywheel system.

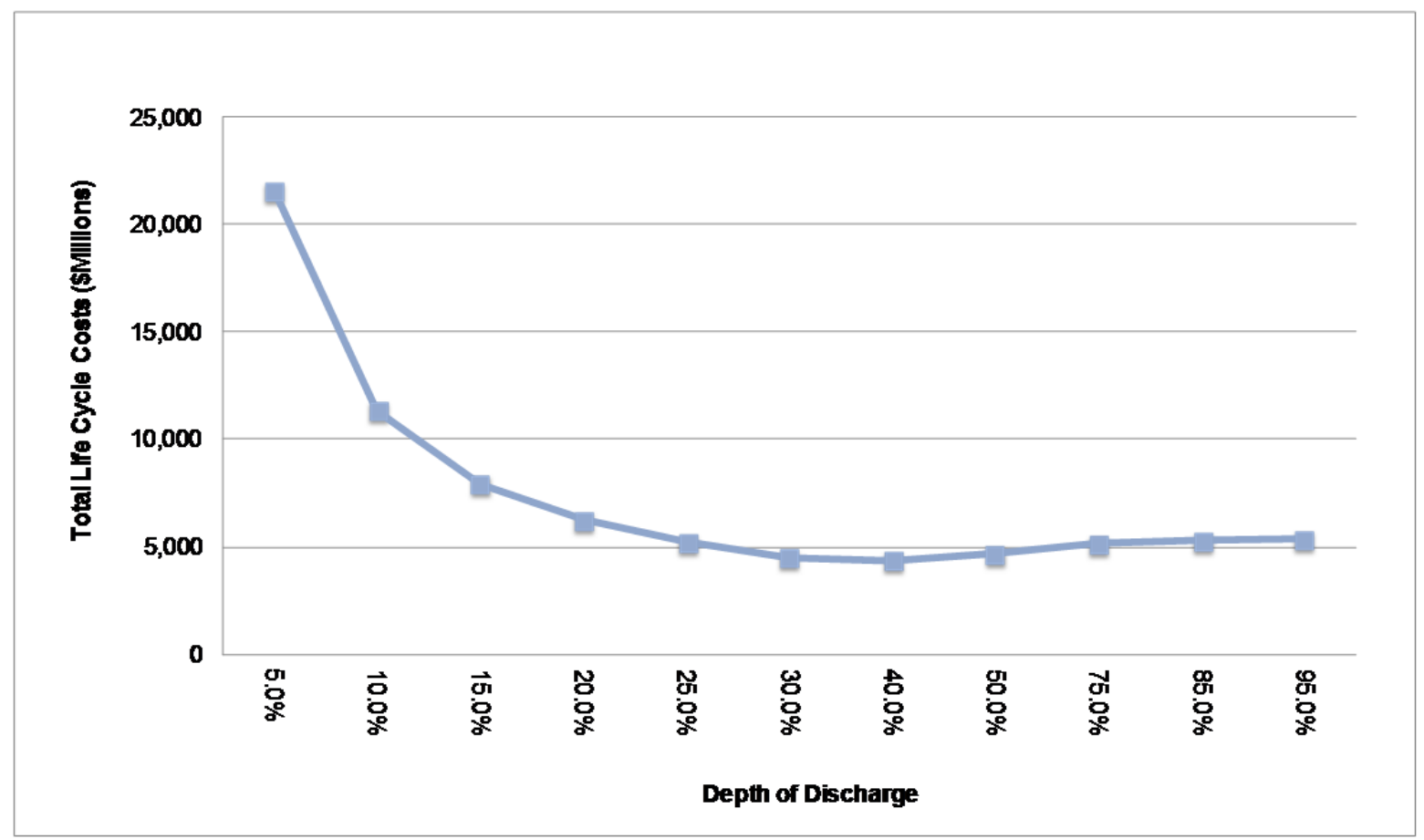

Figure 6.1. Case 3 LCC Estimates

As shown, this figure demonstrates a minimum in the LCC (objective function) is achieved at $40 \%$ under Case 3. This means that over-sizing the Na-S storage device by a factor of $2.5(1 / 0.40)$ would yield the lowest LCC given all of the other assumptions made. It should also be noted that the life-cycle costs remain rather flat over a wide range of DODs (25\% to 100\%) indicating that the LCC is fairly robust against changes in the size of the storage system. This finding suggests that the interim capital cost reductions realized by upsizing the system in order to extend the battery life cycle is largely if not entirely offset by additional initial capital costs. The curve, which rises when the DOD falls below $40 \%$, does so because we assume a 10-year economic life for Li-ion batteries regardless of use. Thus, upsizing the battery fails to extend the life cycle beyond the 10-year maximum when DODs fall below $40 \%$. This is of significant importance for long-term infrastructure planning investments. It indicates that over the longterm an investment in battery storage with a DOD-dependent lifetime is relatively insensitive to oversizing.

For each of the nine cases, costs are computed for battery DODs ranging from 5\% to $95 \%$. Results for the cost minimizing DOD for each case are presented in Table 6.3. As shown, the cost minimizing 
DODs fall between $20 \%$ and $40 \%$ for cases 2 and 3 . For the other cases considered in this analysis, the cost minimizing DOD ranges between 85\% and 95\%. With the exception of case 6 , the higher DOD under these scenarios is due to the heavy reliance on PH or CAES with minimal demand placed on the battery systems. In the absence of heavy use, these systems can withstand higher depths of discharge. For case 6 , the higher depth of discharge is due to the ability of the redox flow battery to withstand higher levels of usage (DOD) with minimal deterioration.

Table 6.3. Cost Minimizing DOD, Battery Capacity, and Economic Life by Case

\begin{tabular}{cccc}
\hline Case & DOD & $\begin{array}{c}\text { Battery Capacity } \\
(\text { MWh })\end{array}$ & Economic Life $^{(\mathrm{a})}$ \\
\hline 1 & -- & -- & -- \\
2 & $30 \%$ & 1,978 & 13.0 \\
3 & $40 \%$ & 1,468 & 7.5 \\
4 & $95 \%$ & 593 & 25.0 \\
5 & -- & -- & - \\
6 & $95 \%$ & 650 & 25.0 \\
7 & $85 \%$ & 162 & 12.9 \\
8 & $95 \%$ & 51 & 13.0 \\
9 & -- & -- & -- \\
\hline
\end{tabular}

(a) The maximum battery life for flywheels and the redox flow batteries is 25 years. The maximum battery life for Na-S and Li-ion batteries is 13 and 10 years, respectively.

\subsection{Economic Parameters}

The cost framework outlined in Section 4 and the cost model supporting this research rely on a number of assumptions regarding major cost elements, including capital costs, O\&M costs, fuel costs, and emissions costs. Costs are segmented according to each of these four cost categories within each of the aforementioned nine technology cases. The remainder of this section details the assumptions underlying each cost component.

\subsubsection{Capital Costs}

Section 3.3 presents capital cost estimates for each technology, and documents the basis for each estimate. Based on the economic lives of each technology, interim capital costs are incurred as necessary to provide required service over the 50-year analysis time horizon. For each option, results are examined using forecasted 2020 prices. In all cases, with the exception of sensitivity analyses presented in Section 5.3, present value costs of investments made in future years are discounted at a nominal rate of $8.0 \%$.

The bases of the capital costs associated with each battery technology, as well as flywheels, CAES and $\mathrm{PH}$, are outlined in Section 3.3 and Appendix A. In addition to these capital costs, one case considers the capital costs of CT and another case includes the capital costs associated with DR. Note that the costs of implementing DR are assumed to be $\$ 50.70$ per $\mathrm{kW}$ per year (EPRI 2009). Over 50 years, the present 
value of DR capital costs is $\$ 620$ per $\mathrm{kW}$, discounted at $8.0 \%$. Combustion turbine capital costs are estimated at \$990 per kW based on the latest estimates presented in the 2011 AEO (DOE/EIA 2011). In addition to these costs, PCS and BOP capital costs are included in all cases involving battery installations, and are estimated at $\$ 150$ per $\mathrm{kW}$ and $\$ 50$ per $\mathrm{kW}$, respectively (see Table 4.2). No additional CC power plant capacity is required to meet the energy requirements set forth in this assessment. Therefore, CC plant capital costs are excluded from the analysis. The costs of operating those CC plants, however, are included in the cost estimates presented for each case.

\subsubsection{Operations and Maintenance Costs}

For combustion and CC turbines, O\&M costs are expressed in variable terms based on data presented in the 2011 AEO. For CT, fixed O\&M costs are $\$ 10.24$ per $\mathrm{kW}$ and variable O\&M costs are $\$ 8.56$ per MWh. CC O\&M costs are estimated at \$14.93 per kW and \$3.54 per MWh for fixed and variable, respectively (DOE/EIA 2011).

For batteries technologies, O\&M costs were also split into fixed and variable components. The fixed component is incurred every year regardless of the energy requirement, while the variable component is proportional to electrical energy (kWh) throughput. Fixed O\&M costs were estimated at $\$ 3.00$ per $\mathrm{kWh}$ of energy storage capacity while variable O\&M costs were estimated at $\$ .007$ per $\mathrm{kWh}$ for Na-S and Li-ion batteries. These costs are detailed in Section 3.3. In addition to these costs, PCS O\&M costs are included and estimated at \$2 per kW of installed capacity for Na-S, Li-ion, and redox flow batteries.

Pumped hydro, CAES, flywheel, and redox flow battery O\&M costs are expressed in terms of fixed and variable components as well. Fixed O\&M costs for PH, CAES, flywheel, and redox flow batteries are estimated at $\$ 4.60, \$ 7.00, \$ 18.00$, and $\$ 5.00$ per $\mathrm{kW}$ of installed capacity, respectively. Variable O\&M costs are estimated at \$.004, \$.003, \$.001, and \$.001 per kWh of throughput for PH, CAES, flywheel, and redox flow batteries, respectively.

\subsubsection{Fuel Costs}

Fuel costs for each alternative were developed using average daily energy requirements as measured in million British Thermal Units (MMBTU). These energy requirements were generated based on the CT and CC turbine production schedules designed to meet load balancing requirements for the four WECC sub-regions in 2020 .

In each scenario, fuel costs associated with CT alternatives are higher than those estimated for each of the CC turbine, CAES, flywheel, PH, or battery alternatives. Fuel cost differentials are due to varying heat rates, which are a function of energy efficiencies. The energy requirements of the CC plus battery alternatives were calculated as the product of the efficiency levels associated with each component.

Average daily energy requirements were expanded to annual energy requirements, which were in turn multiplied by natural gas prices ( $\$ 4.94$ per MMBTU in 2011 escalated at 3.2\% over the 50-year analysis time horizon) to compute annual fuel costs for each alternative (DOE/EIA 2011). 


\subsubsection{Emissions Costs}

Fuel combustion levels assigned through the approach described previously were used to establish emissions levels through the application of EPA coefficients for converting quadrillion Btus into metric tons, as outlined in Table 6.4 (EPA 1995). These emissions levels were, in turn, used to construct emissions cost estimates.

Prices for emissions allowances for $\mathrm{NO}_{\mathrm{x}}, \mathrm{SO}_{2}$, and $\mathrm{CO}_{2}$ are presented in Table 6.4. $\mathrm{NO}_{\mathrm{x}}$ prices were obtained from the January-February $2010 \mathrm{NO}_{\mathrm{x}}$ Market Monthly Market Update (annual $\mathrm{No}_{\mathrm{x}}$ allowances) published by Evolution Markets (Evolution Markets 2010). SO $\mathrm{SO}_{2}$ prices were also obtained through Evolution Markets in the January-February $2010 \mathrm{SO}_{2}$ Monthly Market Update. Prices for $\mathrm{CO}_{2}$ allowances (\$45 per ton) were derived from the Sixth Northwest Power Plan (Northwest Power and Conservation Council 2010).

Table 6.4. Emissions Cost Data

\begin{tabular}{ccccc}
\hline Emissions Data & & $\mathrm{SO}_{2}$ & $\mathrm{NO}_{\mathrm{x}}$ & $\mathrm{CO}_{2}$ \\
\hline Allowances & $\$$ /metric ton & $\$ 71.75$ & $\$ 600$ & $\$ 45$ \\
EPA Coefficients & Metric tons/quadrillion Btu & 267 & 978 & $53,060,000$ \\
\hline
\end{tabular}

\subsection{Results}

The economic assessment methodology detailed in the preceding section of this report was used to compute cost estimates for nine cases using combinations of several energy generation or storage technologies - CTs, CC, Na-S and Li-ion batteries, PH, CAES, flywheels, redox flow batteries, and DR. These cases are defined in Section 5.1. For each case, the objective was to meet the load balancing requirements for WECC sub-regions over a 50-year time horizon.

The results of the economic analysis for the NWPP using 2020 prices are presented in Table 6.5 and Figure 6.2. These results represent the base or reference case. Detailed results by WECC sub-region are presented in Section 7. Sensitivity analysis results are presented in Section 6.5. Of the nine cases examined, Case 2, which employs Na-S batteries plus CC plants, is the least cost alternative at \$2.8 billion. Note that the values presented in Table 6.5 represent the present value of the stream of capital, O\&M, fuel, and emissions costs over a 50-year time horizon. The escalation rate used for both capital and $\mathrm{O} \& \mathrm{M}$ costs is $1.8 \%$ and we used an $8 \%$ nominal discount rate.

Case 4, which consists of flywheels plus CC plants, represents the second least cost alternative with costs estimated at $\$ 3.3$ billion or $16.7 \%$ higher than those estimated for Case 2 . The costs associated with the DR-only case (Case 9) are more than twice as expensive as those estimated for the two aforementioned cases, registering at $\$ 6.9$ billion. The CAES case (Case 5) is also more expensive with estimated costs of $\$ 9.3$ billion. In the predominantly $\mathrm{PH}$ case with two mode changes per day (Case 8), total costs are estimated at $\$ 13.2$ billion. Total costs under Case 6, redox flow batteries plus CC plants, are estimated at $\$ 6.2$ billion. 
Table 6.5. Economic Analysis Results (in Million 2011 Dollars)

\begin{tabular}{crrrrr}
\hline Case & Capital & Fuel & O\&M & Emissions & Total \\
\hline 1 & 5,175 & 1,067 & 454 & 422 & $\mathbf{7 , 1 1 7}$ \\
2 & 2,316 & 164 & 304 & 65 & $\mathbf{2 , 8 4 9}$ \\
3 & 3,884 & 147 & 277 & 58 & $\mathbf{4 , 3 6 6}$ \\
4 & 2,635 & 70 & 592 & 28 & $\mathbf{3 , 3 2 4}$ \\
5 & 6,332 & 1,478 & 903 & 584 & $\mathbf{9 , 2 9 8}$ \\
6 & 5,629 & 190 & 272 & 75 & $\mathbf{6 , 1 6 6}$ \\
7 & 6,334 & 144 & 281 & 57 & $\mathbf{6 , 8 1 7}$ \\
8 & 11,550 & 646 & 776 & 255 & $\mathbf{1 3 , 2 2 7}$ \\
9 & 6,891 & - & - & - & $\mathbf{6 , 8 9 1}$ \\
\hline
\end{tabular}

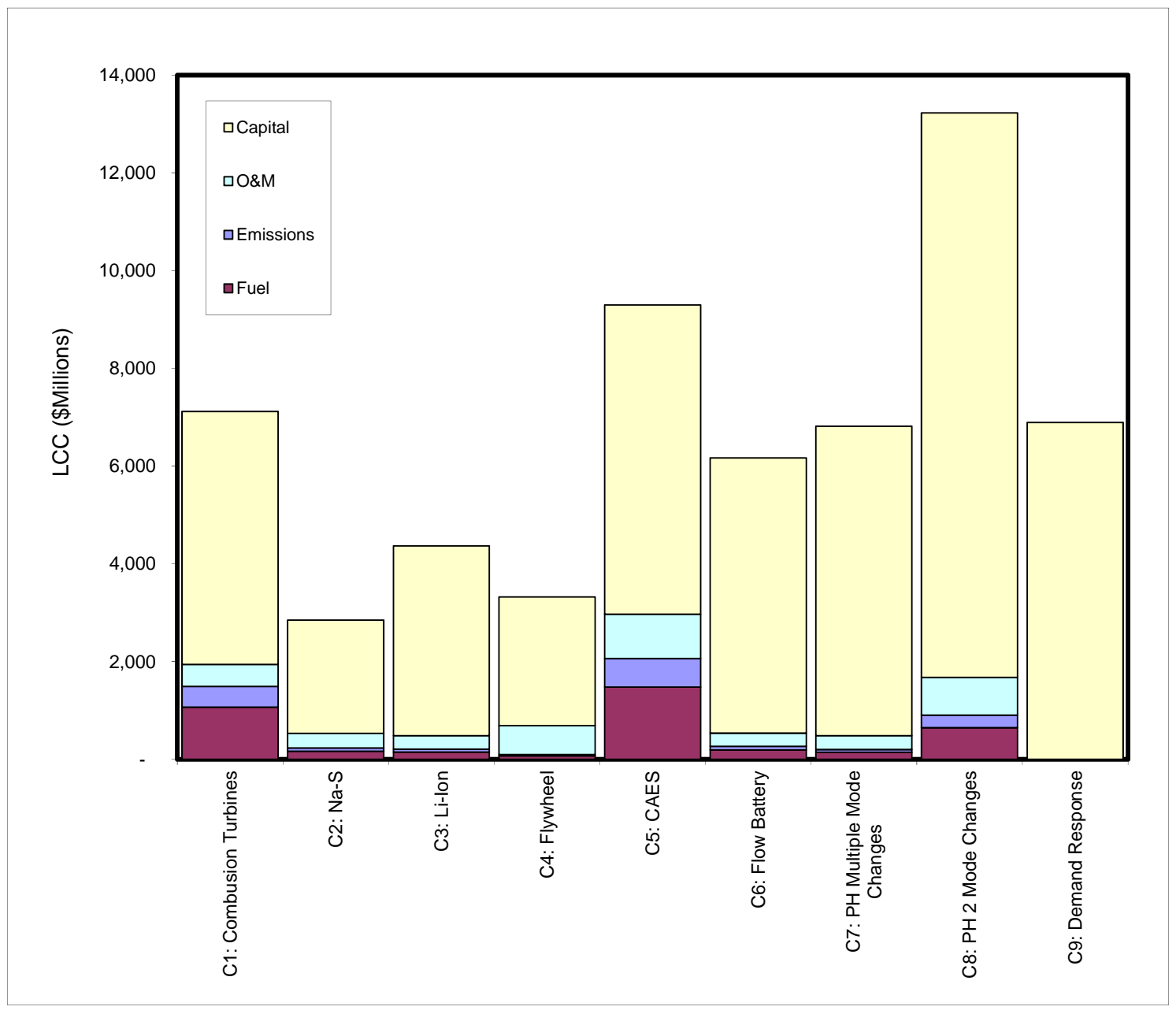

Figure 6.2. Scenario LCC Estimates

In nearly all cases, the costs associated with the energy storage options are lower than those estimated for the combustion turbine case (Case 1), particularly with respect to fuel and emissions costs. Under the current scenarios, capital costs drive the outcome and the CT and PH with their corresponding high 
capital costs do not perform well. Both options appear ill-suited for providing balancing services alone. Both options appear ill-suited for providing balancing services alone. For example, PH with its large reservoir is underutilized in this analysis.

The base or reference case analysis uses 2020 price forecasts. Over the next decade, conventional technologies are forecast to hold steady or increase in cost due to tight global steel and resource markets while new technologies' costs are forecast to decline as they mature. As shown in the next section of this report, using current 2011 price estimates favors mature technologies.

Table 6.6 shows the LCC results of all nine technology cases to meet only new load and wind capacity forecast for 2020. This set of cases or scenario assumes that uncertainty tied to existing load and wind capacity is addressed using existing grid assets. The relative cost ranges of the energy storage cases under this alternative scenario does not vary significantly, though Case 3 (Li-ion) is relatively more costeffective when compared to the base case as it is less costly than the flywheel case (Case 4) in the NWPP, RMPP, and CAMX sub-regions. The costs drop significantly for each sub-region with the exception of the RMPP. The additional load of the RMPP is a significant portion of the total demand. According to the TEPPC electricity demand projection and 2010 BA load provided by the WECC, the load growth for the RMPP is forecast at $23 \%$ compared to $14 \%$ for the AZNMNV, $2 \%$ for the CAMX, and $6 \%$ for the NWPP.

Table 6.6. Economic Analysis Results - Base Case vs. New Load and Wind Capacity Case (in million 2011 dollars)

\begin{tabular}{crrrc|rrrr}
\hline \multirow{2}{*}{$\begin{array}{c}\text { Energy } \\
\text { Storage } \\
\text { Case }\end{array}$} & \multicolumn{4}{c|}{ Basecase } & \multicolumn{3}{c}{ New Load and Wind Capacity Case } \\
\cline { 2 - 8 } & NWPP & RMPP & CAMX & AZNMNV & NWPP & RMPP & CAMX & AZNMNV \\
\hline 1 & 7,117 & 2,678 & 8,463 & 4,567 & 1,016 & 1,957 & 1,953 & 1,007 \\
2 & 2,849 & 944 & 3,499 & 1,862 & 324 & 551 & 568 & 279 \\
3 & 4,366 & 1,401 & 5,168 & 2,778 & 464 & 790 & 819 & 412 \\
4 & 3,324 & 1,134 & 3,939 & 2,041 & 469 & 846 & 873 & 347 \\
5 & 9,298 & 3,317 & 10,439 & 5,871 & 1,160 & 2,174 & 2,030 & 908 \\
6 & 6,166 & 2,056 & 7,344 & 3,800 & 863 & 1,524 & 1,575 & 643 \\
7 & 6,817 & 2,348 & 8,056 & 4,158 & 935 & 1,669 & 1,724 & 698 \\
8 & 13,227 & 4,627 & 14,277 & 7,994 & 1,722 & 3,306 & 3,074 & 1,259 \\
9 & 6,891 & 2,277 & 8,120 & 4,154 & 968 & 1,660 & 1,467 & 715 \\
\hline
\end{tabular}

The results of this analysis, which support the application of energy storage technologies for balancing services, could lead the reader to question why these technologies have not yet been deployed on a broader scale. While the results of this analysis suggest perhaps that they should be deployed more broadly, there are technical and financial barriers worth discussing.

The first hurdle to broader deployment is tied to technical limitations and inexperience in using energy storage technologies for balancing and other ancillary services. The small number of installations highlighted in Appendix A, while certain to provide useful technical information for utilities considering adoption of energy storage, does not constitute a proven track record sufficient to demonstrate the 
feasibility of implementation on a broader scale. Furthermore, recent installations have presented technical challenges resulting in significant one-time engineering time and cost that were not considered in the LCC analysis.

There is also a significant degree of cost uncertainty associated with the energy storage technologies considered in this study. Project information and literature reviewed for this study present a wide range of costs for Na-S, Li-ion, and redox flow batteries, as well as flywheels and CAES. In some cases, capital costs varied significantly, resulting in LCC variability of up to $48 \%$. Table 6.7 presents low- and highend cost estimates for each of the technologies examined in this study for the year 2020 and Figure 6.3 presents the effects of capital cost variation on the total LCC for each case.

Table 6.7. Capital Cost Range for Various Technologies in Year 2020

\begin{tabular}{lrr}
\hline \multicolumn{1}{c}{ Technology } & \$/kWh & \multicolumn{1}{c}{$\$ / \mathrm{kW}$} \\
\hline Sodium sulfur & $181-331$ & \\
Lithium-ion & $290-700$ & \\
Pumped Hydro & 10 & $1,640-2,400$ \\
Combustion Turbine & & 990 \\
Demand Response & & 620 \\
Compressed Air & 3 & $500-1,140$ \\
Flywheels & $81-148$ & $200-820$ \\
Redox Flow Battery & $88-173$ & $608-942$ \\
\hline
\end{tabular}

The technical and cost uncertainty regarding energy storage technologies is further evaluated in the TRLs and MRLs assigned to each and presented in Table 4.2. TRLs assigned to the energy storage technologies are as low as six for redox flow batteries and seven for $\mathrm{Na}-\mathrm{S}$ batteries, Li-ion batteries, and flywheels. A TRL of six indicates that a prototype system has been verified while a TRL of seven indicates that an integrated pilot system has been demonstrated. Conversely, CT received a TRL of nine, which indicates that the system is proven and ready for full commercial deployment. The MRL for flywheels and redox flow batteries is five indicating that the manufacturing process is under development. $\mathrm{Na}-\mathrm{S}$ and Li-ion batteries received MRLs of six indicating that a critical manufacturing process for utilityscale systems has been prototyped. CT received an MRL rating of 10 indicating that full rate production has been demonstrated and lean production practices are in place.

The nascent state of deployment in which most of these energy storage technologies currently are combined with the corresponding technical and cost uncertainty could present problems from the standpoint of regulatory recovery and financing costs. If these investments were undertaken using project-level financing and cost uncertainty drove up borrowing costs by 200 basis points, or $2 \%$, the LCC for Case 3 (Li-ion batteries and CC power plants) would increase by $21.5 \%$ from $\$ 4.4$ billion to $\$ 5.3$ billion. However, the impact would be more likely to be indirect as utilities perform investment planning that effects their overall creditworthiness and ability to make a rate case to utility commissions. 


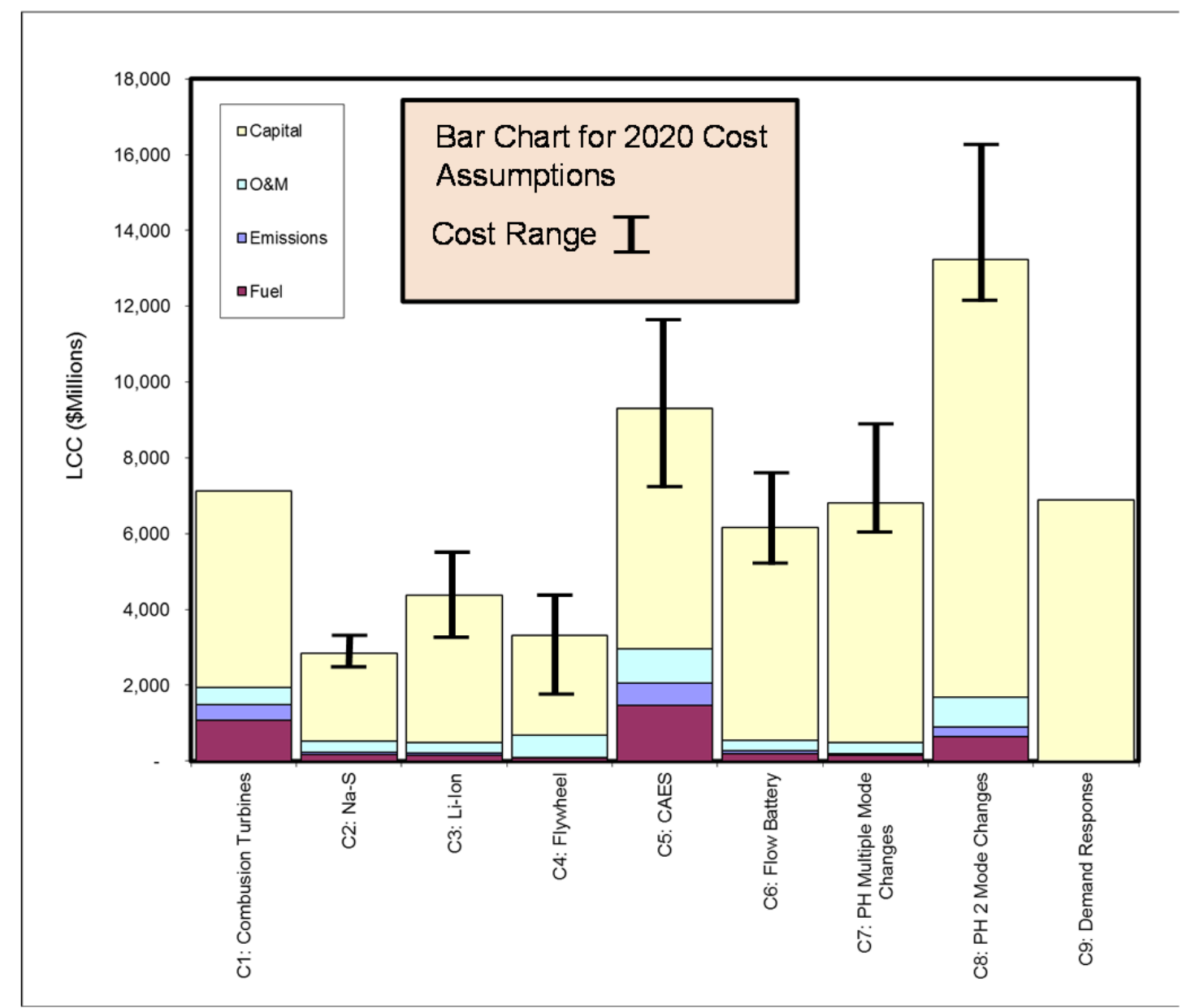

Figure 6.3. LCC Estimates Adjusted due to Capital Cost Variability

\subsection{Sensitivity Analysis}

To explore the sensitivity of the results to varying a small number of key assumptions, the research team conducted a series of sensitivity analyses. Sensitivity analysis was performed by making the following adjustments to the assumptions underlying the results:

SA 1. Use prices for each technology estimated for 2011

SA 2. Vary the discount rate between $6 \%$ and $10 \%$

SA 3. Increase the annual capital cost escalation rate to $3 \%$

SA 4. Increase the annual fuel price escalation rate to $5 \%$

SA 5. Vary the capital cost assumptions using the low- and high-end of the cost range for each technology presented in Table 6.7.

Table 6.8 presents the results of the five sensitivity analyses as compared to the base case for the NWPP. Note that both the second and fifth sensitivity analyses, which respectively adjust discount rates and capital cost assumptions, evaluate two alternative assumptions. 
Table 6.8. Sensitivity Analysis Results

\begin{tabular}{|c|c|c|c|c|c|c|c|c|}
\hline Case & Base Case & SA 1 & $\begin{array}{c}\text { SA } 2 \\
(6 \% \\
\text { discount } \\
\text { rate) }\end{array}$ & $\begin{array}{l}\text { SA } 2 \\
(10 \% \\
\text { discount } \\
\text { rate) }\end{array}$ & SA3 & SA 4 & $\begin{array}{c}\text { SA } 5 \\
\text { (Low) }\end{array}$ & $\begin{array}{c}\text { SA } 5 \\
\text { (High) }\end{array}$ \\
\hline 1 & 7,117 & 7,216 & 9,067 & 5,986 & 7,787 & 7,539 & 7,117 & 7,117 \\
\hline 2 & 2,849 & 3,916 & 3,489 & 2,469 & 3,096 & 2,914 & 2,252 & 3,074 \\
\hline 3 & 4,366 & 7,781 & 5,495 & 3,691 & 4,909 & 4,424 & 3,003 & 5,542 \\
\hline 4 & 3,324 & 6,029 & 3,897 & 2,984 & 3,500 & 3,351 & 1,723 & 4,162 \\
\hline 5 & 9,298 & 10,574 & 11,208 & 8,204 & 9,708 & 9,882 & 6,926 & 11,247 \\
\hline 6 & 6,166 & 8,969 & 7,627 & 5,291 & 6,856 & 6,241 & 4,961 & 7,367 \\
\hline 7 & 6,817 & 6,601 & 7,125 & 6,656 & 6,853 & 6,874 & 5,989 & 8,547 \\
\hline 8 & 13,227 & 12,608 & 14,022 & 12,790 & 13,253 & 13,482 & 11,777 & 16,390 \\
\hline 9 & 6,891 & 6,891 & 8,974 & 5,571 & 6,891 & 6,891 & 6,891 & 6,891 \\
\hline
\end{tabular}

In the first sensitivity analysis case, current technology prices were used. The underlying assumption governing this first case is that prices remain unchanged over the next nine years and the cost adjustments forecast in the 2020 base case are never realized. The capital cost forecasts for CT, CC plants and PH were taken from the AEO 2010 reference case, which includes forecast costs out to 2035 (DOE/EIA 2011). The costs for these technologies are forecast to grow over the next 5-6 years because construction costs for all plant types have risen significantly in recent years. This cost growth is forecast based on a commodity cost index that was implemented for the AEO. Growth in the cost index is tied to rising commodity prices, such as steel, copper, cement, and other construction materials. In the long run, the commodity prices do decline and technology growth leads to cost reductions in the latter years of the forecast.

Battery and flywheel costs used in the base case (\$510 per kWh for Li-ion, \$290 per kWh for Na-S, $\$ 133 / \mathrm{kWh}$ and $\$ 775 / \mathrm{kW}$ for redox flow battery, and $\$ 115 / \mathrm{kWh}$ and $\$ 610 / \mathrm{kW}$ for flywheel) reflect significant cost reductions forecast over the 2011 to 2020 time period. When using current cost data for these energy storage technologies, costs increase to \$1,000 per $\mathrm{kWh}$ for $\mathrm{Li}-\mathrm{ion}, \mathbf{\$} 415$ per $\mathrm{kWh}$ for Na-S, \$215 per kWh and \$1,111 per kW for redox flow batteries, and \$148 per kWh and \$1,277 per kW for flywheels.

Due to the forecasts underlying the base case, which include cost reductions for battery technologies, using current prices makes scenarios involving $\mathrm{CT}, \mathrm{PH}$, and DR relatively more cost efficient. For example, PH with multiple mode changes (Case 7) is much more cost-competitive when using 2011 prices with estimated costs dropping to $\$ 6.6$ billion. However, the results of Sensitivity Analysis 1 continue to show Case 2 as the most cost-effective, though costs under this scenario increase significantly from $\$ 2.8$ billion to $\$ 3.9$ billion.

The discount rate was varied in SA 2 from the 8\% used in the base case to $6 \%$ and $10 \%$. By reducing the discount rate, the present value of the interim capital costs associated with battery replacement in the out years of the 50-year analysis time horizon are increased. When the discount rate is reduced, the $\mathrm{PH}$ scenarios become relatively more cost efficient because the asset is long-lived (50 years) and does not require interim capital costs. Reducing the discount rate to $6 \%$ increases the costs associated with Case 2 
significantly (Na-S batteries plus CC) from $\$ 2.8$ billion to $\$ 3.5$ billion (22.4\%). For Case 7 (Na-S batteries plus CC plus $\mathrm{PH}$ with frequent mode changes per day), however, the impact is minimal with costs increasing by $4.5 \%$ from $\$ 6.8$ billion to $\$ 7.1$ billion. Using the higher discount rate of $10 \%$ conversely favors battery technologies with large interim capital costs.

The third and fourth sensitivity analyses, which include variability with respect to cost inflation for capital and fuel costs, with minor exceptions do not appear to lead to a re-ordering of the most cost efficient options. Thus, results do not appear to be very sensitive to varying capital cost and fuel price inflation rates.

The final sensitivity analysis, which examines the impacts of varying the capital cost assumptions presented in Table 6.6, demonstrates that some cases have more variability or uncertainty than others. More specifically, the cost variance is far greater among the less mature energy storage technologies, including $\mathrm{Na}-\mathrm{S}$, Li-ion, and redox flow batteries, as well as flywheels and CAES. For these technologies, the projects and literature reviewed for this study demonstrates that costs could vary significantly. For example, using the upper bound of the Li-ion capital cost range would increase total costs for Case 3 by $27 \%$. Using the lower bound of the flywheel capital cost range, on the other hand, would reduce the total costs associated with Case 4 by $48 \%$. The range of plausible capital costs is much smaller for more mature technologies, including CT, CC plants, and PH. 


\subsection{Arbitrage Opportunities for Energy Storage}

Arbitrage is the practice of taking advantage of price differences between two market prices. In the context of electric energy markets, energy storage can be used to charge during low-price periods (i.e., buying electricity) in order to discharge the stored energy during periods of high prices (i.e., selling during high-priced periods). The economic reward is the differential in the prices between buying and selling electrical energy, minus the losses during the full charging/discharging cycle.

The revenue potential of arbitrage is illustrated in Figures 7.1a and 7.1b. Figure 7.1a presents an illustrative LMP differential for hours throughout the year along a congested point in the grid. Note that the number of hours in the year was cut in half to account for the time required to charge energy storage devices. LMP differentials begin at high levels yielding the largest marginal revenues but would be expected to decline as more energy storage enters the arbitrage market. Figure $7.1 \mathrm{~b}$ demonstrates how the marginal revenue generated for each additional hour of operation of a power plant, or in this case an energy storage device, would be expected to decline. As these energy storage devices are expanded in terms of capacity and production, marginal revenues per MWh would be expected to decline until marginal revenues and the marginal costs of introducing more capacity reach a point of equilibrium. At this point, no more capacity would enter the market because the marginal expansion in energy capacity would yield economic losses.

The figures demonstrate that the first block of installed energy storage capacity would supply a portion of the energy required during the highest value hours (Area A). At the height of the load duration curve when demand is greatest, the LMP differential would reach an apex and the revenue per hour of operating the energy storage device would be maximized. In this case, the presence of the first block of energy storage devices entering the market would meet some of the demand placed on the system during the highest peak hours of the year resulting in a reduction in the LMP differential available for other energy suppliers in the arbitrage market (Area B). In addition to capturing the high-value hours represented by Area A in the figures, building energy storage into the market would result in a shift in the LMP differential curve further reducing profits available to other market participants or to further expansion of energy storage capacity.

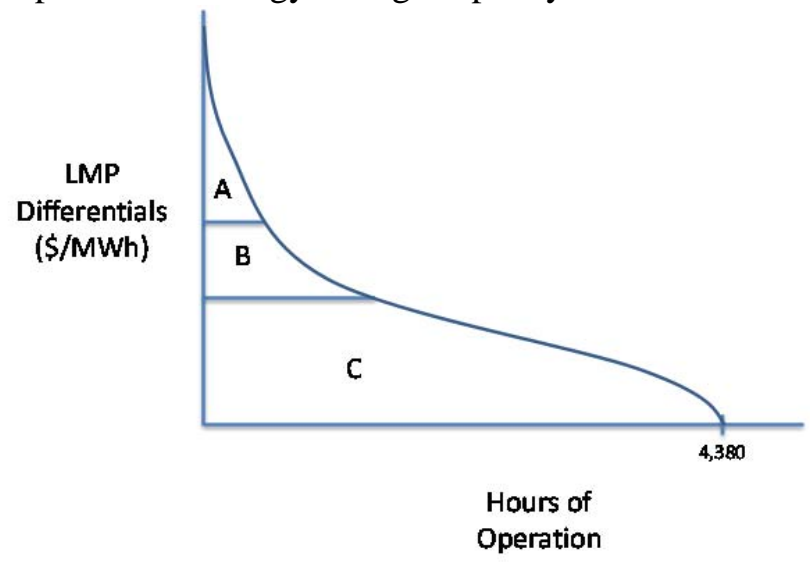

(a)

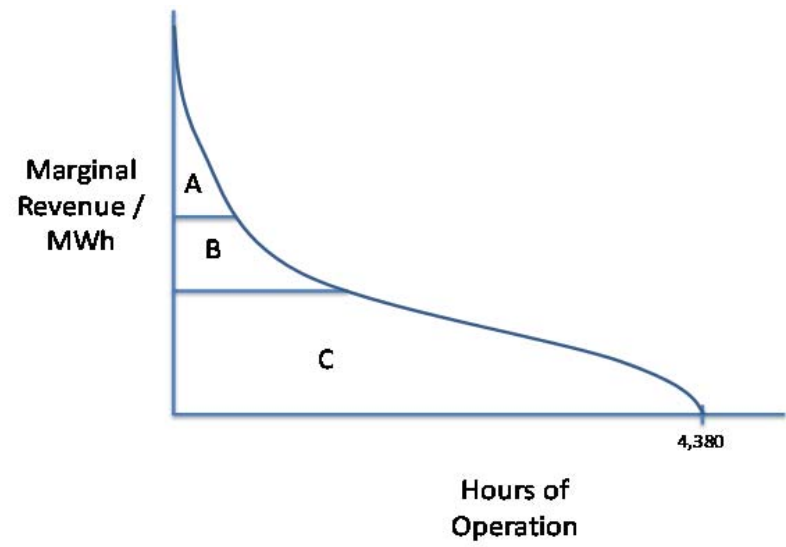

(b)

Figure 7.1. (a) LMP Differential per Hour of Operation (\$/MWh) and (b) Marginal Revenue per Hour of Operation (\$/MWh) 
To generate arbitrage revenue, energy storage is cycled daily by charging during off-peak hours and discharging during peak hours of the day. Electro-chemical energy storage systems used for arbitrage store and discharge electrical energy at a specified power rating for many hours (4-10 hours or more). $\mathrm{PH}$ storage systems with a sufficiently large reservoir, however, could be used to store capacity over longer durations. For example, PH storage could be drawn down to a low state of charge by Friday evening. In turn, pumping could be initiated from Friday night through the weekend to be terminated in the early morning hours on Monday. In this instance, PH could then be used to supply energy during peak weekday hours. In some cases, it may be desirable to store electrical energy for longer periods. For instance, in the Pacific Northwest where there is significant hydro power capacity along the Columbia and Snake River systems, a rapid snow melt could result in energy production by dams that exceed the region's demand for electricity. In such a case it may be desirable to store the water or the electricity for periods in the summer when the water resources are less ample. Such a storage system would be large in its capacity to store energy for weeks if not months. Typically, for longer duration applications in which significant amounts of energy must be stored, PH, CAES systems, and some electro-chemical storage systems (such as redox flow batteries) are used.

While these energy storage systems have been limited in terms of their overall application, their continued development has been supported through significant public and private investment. For example, the American Recovery and Reinvestment Act (ARRA) is currently funding grid-connected energy storage demonstrations at a federal funding level of approximately $\$ 185$ million with additional industry cost-sharing of roughly $\$ 586$ million. ${ }^{12}$ Of the 16 demonstration projects initiated, eight projects demonstrate or explore storage with a cycling duration of 4 hours or more. The applications of the demonstration projects vary but all of them are capable or designed to perform energy arbitrage functions.

In addition to the energy arbitrage potential, energy storage can provide operating reserves (contingency reserves) and system balancing services to the grid because of its fast response characteristics. Its competitiveness among other technology options has been discussed for the system balancing service in the previous sections of this report. Thus, this section focuses on the cost effectiveness of using energy storage as an arbitrage instrument to mitigate congestion-induced high electricity prices and/or to reduce potential low load conditions in cases where there is insufficient load (commonly at night) coincident with large electricity production attributable to growing wind generation capacity.

The exclusion of the balancing service value is made primarily based on the difficulty of valuing ancillary service in conjunction with the energy arbitrage as a bundled service. To value a bundled storage product would require a coupled optimization in which the market values of ancillary services and energy markets in a given market (hour-ahead or day-ahead markets) must be solved subject to the important constraint of a finite stored energy and a maximum capacity. Due to its analytical complexity, this element has been left to future phases of this research program.

\footnotetext{
${ }^{12}$ Sandia National Laboratories ARRA Energy Storage Demonstrations Webpage. http://www.sandia.gov/ess/docs/ARRA_StorDemos_4-22-11.pdf. Last accessed on September 28, 2011.
} 


\subsection{Arbitrage Analysis Framework}

\subsubsection{General discussion}

To quantify the arbitrage value of energy storage, the research team applied an economic viability approach that compares the annual revenue requirements from the capital expenditure to the revenue potential from arbitrage. As indicated above, the arbitrage value was isolated and de-coupled from any other services that could potentially be bundled. Because of the complexity of the analysis to estimate the total value of bundled services with multiple payment streams, this analysis strictly focused on the arbitrage potential.

Economic viability of an energy arbitrage product or services is defined by a positive cash flow when the net revenue from performing energy arbitrage services exceeds the capital cost recovery requirements. The following equation defines the key parameters for the cash flow:

$$
-\left(P d_{o} p_{o}\right)+\left(P \eta d_{o} p_{p}\right) D>\left(P C_{S t o} d+P C_{P C S}\right) \alpha
$$

where

$$
\begin{aligned}
P & =\text { Power capacity of storage in }[\mathrm{kW}] \\
\eta & =\text { roundtrip efficiency of energy storage in }[-] \\
d & =\text { duration of storage to maintain power output at rated capacity } P \text { in }[\mathrm{h}] \\
d_{o} & =\text { duration of storage operation per day at rated capacity } P \text { in }[\mathrm{h} / \mathrm{day}] \\
D & =\text { number of days per year storage operates [1/year] } \\
p_{o} & =\text { off peak price in }[\$ / \mathrm{MWh}] \\
p_{p} & =\text { off peak price in }[\$ / \mathrm{MWh}] \\
C_{S t o} & =\text { incremental cost of storage device associated with the storage of electric energy in } \\
C_{P C S} & =\text { incremental cost of storage device associated with the power electronics in }[\$ / \mathrm{kW}] \\
\alpha & =\text { annualization factor to annualize an investment in }[-] .
\end{aligned}
$$

Assuming that the energy storage system operates every day over its full duration, then $d_{o}=d$, and rearranging to solve for the necessary capital cost $C_{S t o}$, we can write the equation as follows:

$$
p_{o}\left(-1+\eta \frac{p_{p}}{p_{o}}\right) \frac{D}{\alpha}-\frac{C_{P S C}}{d}>C_{S t o}
$$

Assuming that the incremental cost associated with power electronics for the power conditioning system is a known entity, then necessary incremental cost for the storage component is a function of the off-peak power price $p_{o}$, the differential between peak and off-peak prices expressed as ratio $p_{p} / p_{o}$, and the roundtrip efficiency $\eta$, the number of days of operation $D$, and the annualization factor $\alpha$.

Illustrated in Figure 7.2 is the dependency of the incremental cost $C_{S t o}$ on the peak-to-off-peak prices differential and the efficiency $\eta$ assuming that the storage device will be operating 5 days a week for 50 weeks. The annualization factor is consistent with the economic assumptions of discount rates and life cycles as calculated in the balancing services analysis $(\alpha=0.12)$. Furthermore, assumed is an incremental cost for the power electronics (power conditioning system) of $C_{P C S}=\$ 150 / \mathrm{kW}$ consistent with earlier assumptions shown in Table 4.2. 


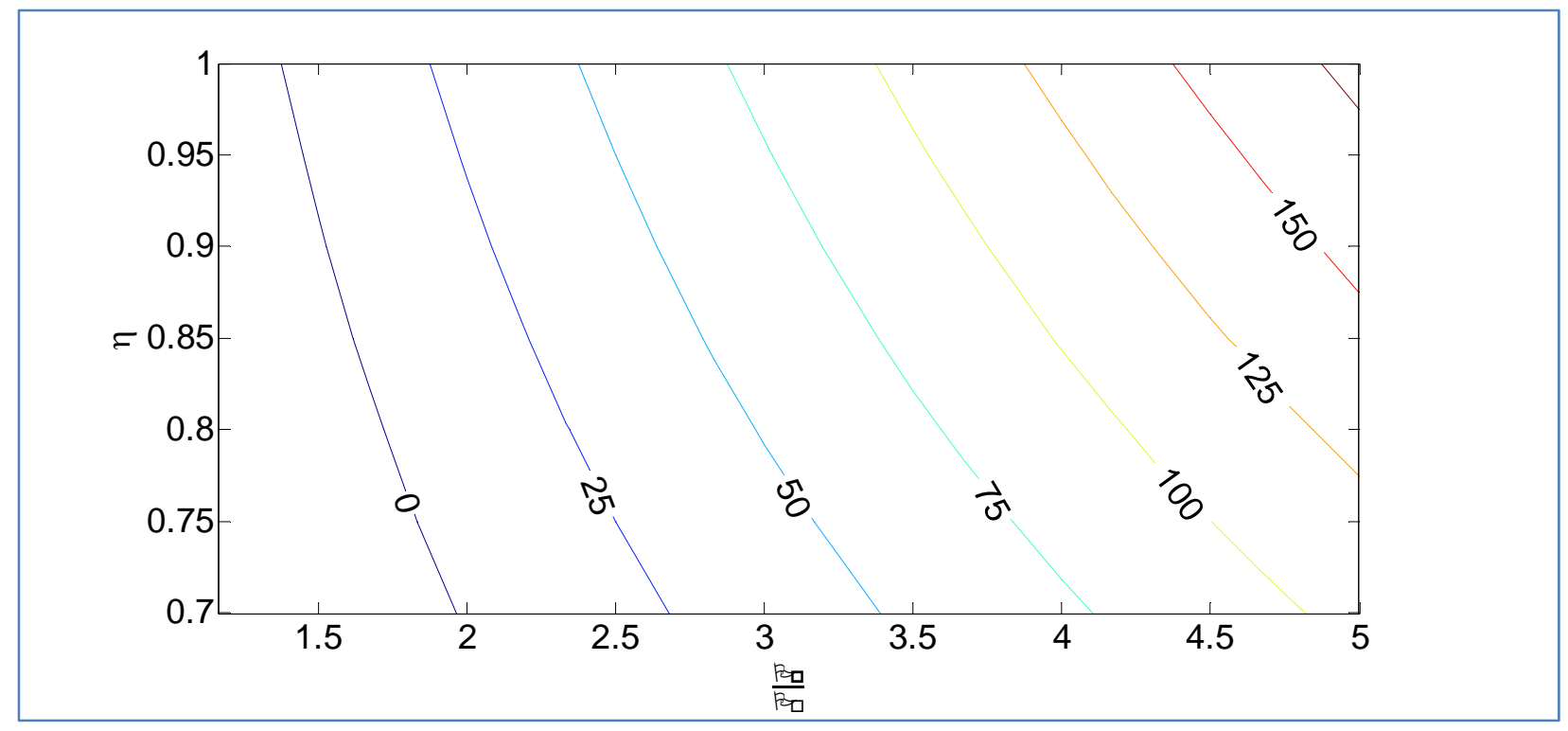

Figure 7.2. Dependency of Capital Cost for Storage Component $C_{\text {sto }}$ in $(\$ / \mathrm{kWh})$ on Peak-Off Peak Ratio and Efficiency. Assumed: $p_{o}=\$ 40 / \mathrm{MWh}, D=260$ days, $\alpha=0.12$.

Figure 7.2 clearly indicates a very low incremental cost for storage component (that scales with kWh) necessary for a positive cash flow, the economic viability criterion. This indicates that for small $p_{p} / p_{o}$ ratios of less than 1.5, which has been observed in competitive market conditions in CAISO for instance, that the breakeven capital cost levels are negative meaning that the power conditioning system by itself is too expensive. This result unequivocally suggests that energy arbitrage by itself is unlikely to be a viable market niche for storage. Rather, it can be a value-enhancing service to other higher valued services.

If we assume that energy storage with sufficient energy capacity, say more than 6 hours, can contribute to the capacity requirements within a balancing authority, then we can assign a capacity value to this resource. Generally, the capacity value or the contribution to the capacity adequacy requirement is determined by its contribution to reduce the loss-of-load-probability. The value of such a resource can be found in active capacity markets such as the New York Independent System Operator (NYISO) installed capacity (ICAP) market. The value range from around $\$ 100 / \mathrm{kW}$-year to close to $\$ 200 / \mathrm{kW}$-year (NYISO 2010). If we assume a $\$ 150 / \mathrm{kW}$-year value that energy storage device with an energy capacity of more than 6 hours can provide, then the additional revenues from capacity payments would increase the target cost to higher values as seen in Figures 7.3 and 7.4. 


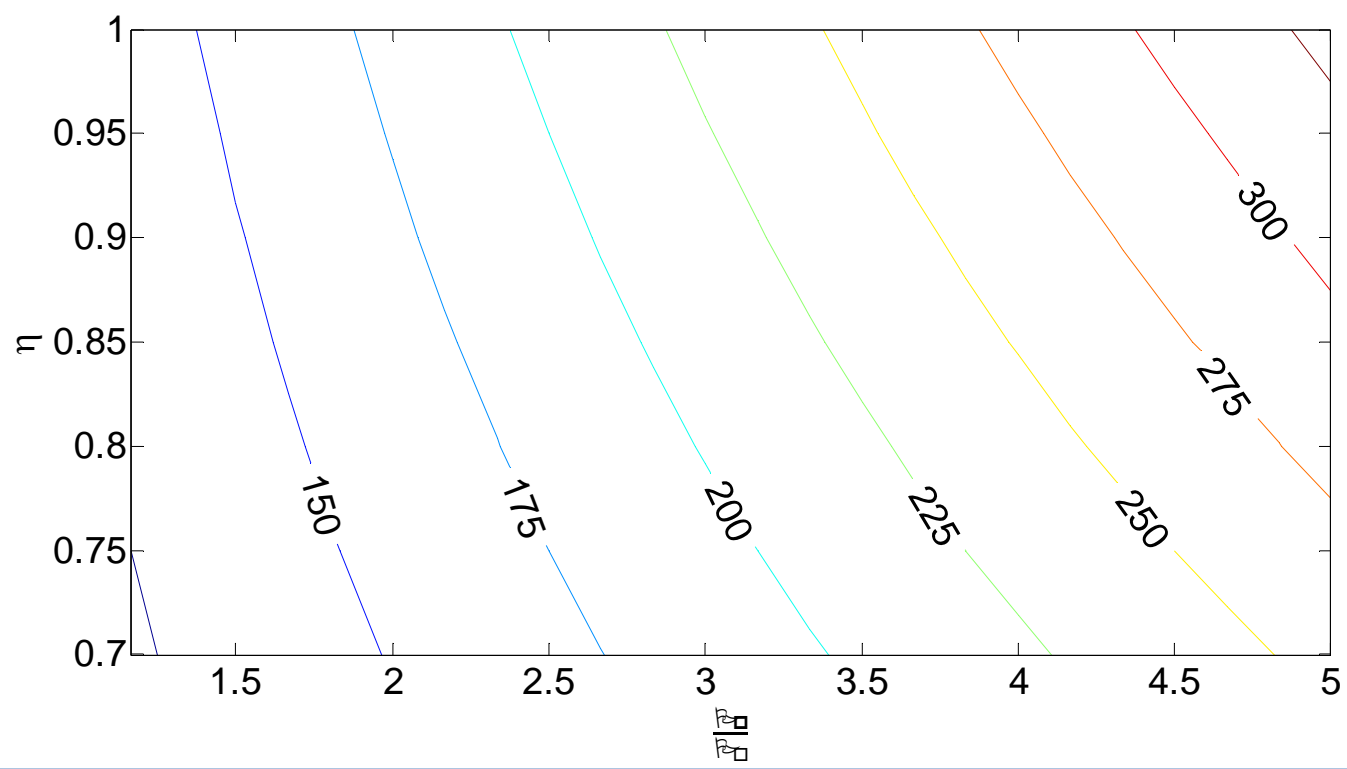

Figure 7.3. Dependency of Capital Cost for Storage Component $C_{\text {sto }}$ in $(\$ / \mathrm{kWh})$ on Peak-Off Peak Ratio and Efficiency. Assumed: $p_{o}=\$ 40 / \mathrm{MWh}, D=260$ days, $\alpha=0.12$, and capacity value of $\$ 150$ per kW per year.

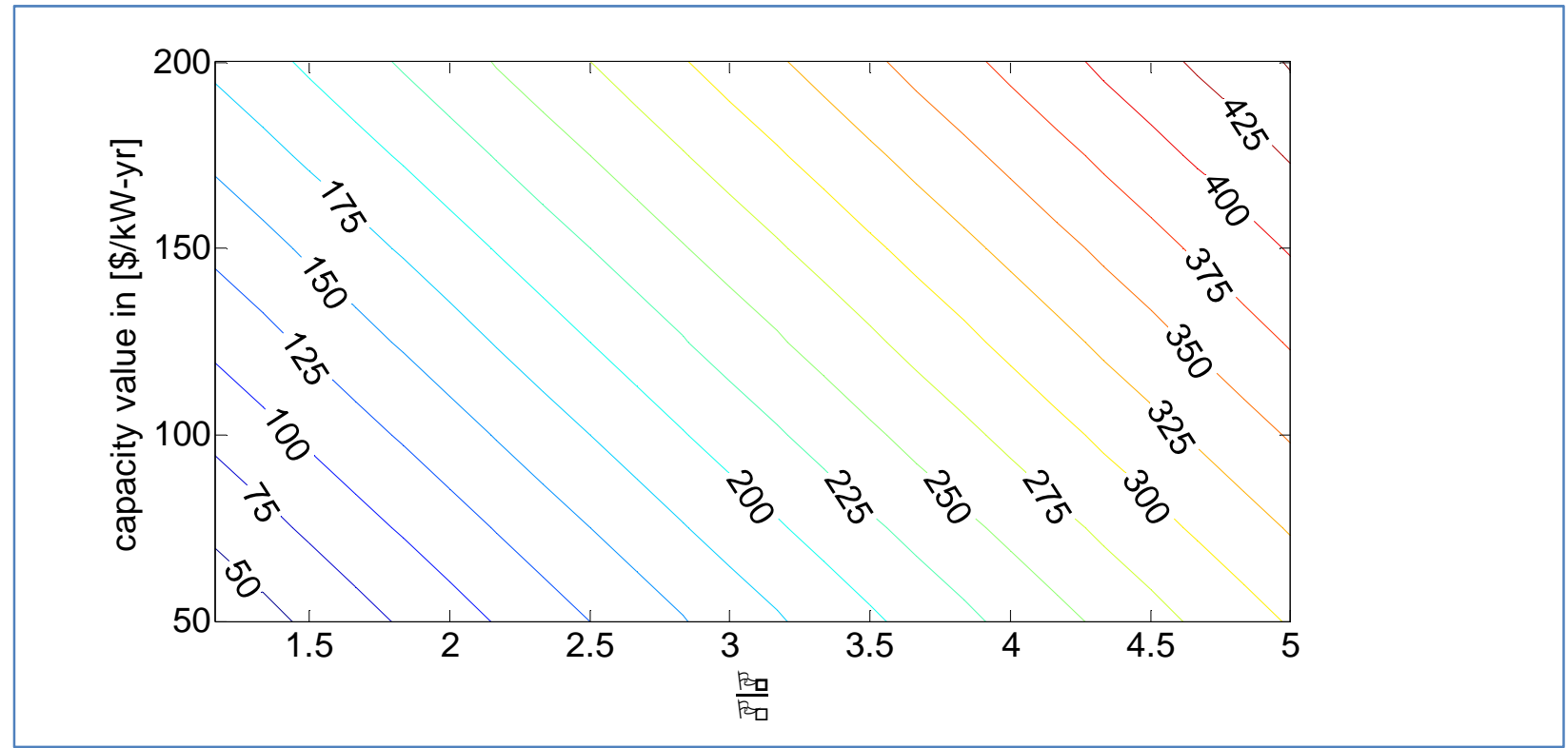

Figure 7.4. Dependency of Capital Cost for Storage Component $C_{\text {sto }}$ in $(\$ / \mathrm{kWh})$ on Peak-Off Peak Ratio and Capacity Value. Assumed: $p_{o}=\$ 40 / \mathrm{MWh}, D=260$ days, $\alpha=0.12$, and efficiency of $\eta=0.85$. 


\subsubsection{Modeled Revenue Estimations}

The revenue potential analysis is based on a production cost approach using PROMOD IV ${ }^{13}$ from Ventyx as an analysis tool. PROMOD is a production cost software that solves security constrained unit commitment and dispatch problems in power systems at either the zonal or nodal transmission level. In this analysis, a WECC system model developed by the vendor was used in its zonal mode with 22 utility zones for the WECC. In this analysis, a 2020 grid scenario is postulated with a generation capacity mix that was based on AEO 2011 reference case (DOE/EIA 2010b).

In support of the arbitrage analysis, several data forecasts were implemented in this model as described briefly below:

- Coal price forecast: The coal price forecast is at the plan level. The prices include historical monthly prices through October 2010 followed by forecast annual prices to the year of study, 2020.

- Natural gas price forecast: Monthly values are forecasted for the year of study.

- Emission price forecast: $\mathrm{NO}_{\mathrm{x}}$ and $\mathrm{SO}_{2}$ forecasts reflect the Federal Clean Air Transportation Rule. Mercury price is not modeled. $\mathrm{CO}_{2}$ price forecasts are included in the model.

- Load forecast: Peak and energy load forecasts are based on the 2009 Federal Energy Regulatory Commission (FERC) 714 filings as well as more recent regional and ISO publications such as the NYISO Gold Book, ISO-NE CELT report, ERCOT long-term reliability assessment, California Energy Commission and similar reports from Canadian sources. Load forecasts are provided at the BA level in the database.

- Topology: The topology modeling is done at the BA level as reported in the 2009 FERC 714 filings. The new topology is designed to better align with ISO regions as well as state boundaries.

- Hydro Energy: Historical average energy used to create the annual and monthly energy forecasts have included 2009 actual data.

- Load Shapes: The synthetic 8,760 load shapes have included hourly loads from 2003-2009.

- Solar hourly shapes: The solar shapes specific to renewable basins are included in the database.

- Zonal Transmission Expansion Plan: Zonal transmission expansion plans includes announced transmission projects and projected projects required to deliver renewable expansion to loads.

- Wind hourly shapes: the wind hourly shapes are derived from NREL wind map data instead of wind data in the provided package.

Our target was to build a system model that reflects the 20\% RPS by the year 2020. Because the database was built with aggressive generation and expansion assumptions, we modified it by reducing generation capacity based on AEO forecasts for the year 2020. The load forecast in the database also uses very high growth assumptions. Thus, we adjusted the demand growth rate to $1.3 \%$ in CAMX and $0.65 \%$ for other areas in the system. In turn, we calculated the existing generation capacity (year 2010) and additional capacity to the year 2020 for the four WECC sub-regions after the generation adjustment.

\footnotetext{
${ }^{13}$ PROMOD IV is an Energy Planning and Analytics Software developed by Ventyx http://www.ventyx.com/analytics/promod.asp.
} 
With the base case determined above, we found that the system always has at least a $45 \%$ reserve margin during the entire year. A system with a 45\% capacity margin, however, leaves little room for profit through arbitrage because energy storage will be competing with other traditional energy generation categories.

To increase the arbitrage value of energy storage, a degraded system was modeled in which we deactivate certain generation categories including CC and CT (and keep other categories unchanged) from the database to lower the reserve margin to 30\%. The generation capacity after CC and CT are removed to achieve the $30 \%$ reserve margin is shown in Table 7.1 . We choose the efficiency of $75 \%$ for $\mathrm{PH}$ storage and $87 \%$ for the battery storage categories under consideration. The efficiency for battery storage is chosen higher in the arbitrage study compared with that in the previous sections (balancing services) because it is cycled at much lower rates. Simulation results for those cases are presented in the next section of this report.

Table 7.1. Existing and Additional Installed Capacity (MW) for AZNM, CAMX, NWPP, and RMPA for the Case of 30\% Reserve Margin

\begin{tabular}{l|r|r|r|r|r|r|r|r}
\hline & \multicolumn{2}{|c|}{ AZNM } & \multicolumn{2}{c|}{ CAMX } & \multicolumn{2}{c|}{ NWPP } & \multicolumn{2}{c}{ RMPA } \\
\cline { 2 - 9 } Category & $\begin{array}{c}\text { Existing } \\
(2010) \\
{[\mathrm{MW}]}\end{array}$ & $\begin{array}{c}\text { New } \\
\text { Capacity } \\
{[\mathrm{MW}]}\end{array}$ & $\begin{array}{c}\text { Existing } \\
(2010) \\
{[\mathrm{MW}]}\end{array}$ & $\begin{array}{c}\text { New } \\
\text { Capacity } \\
{[\text { MW] }}\end{array}$ & $\begin{array}{c}\text { Existing } \\
(2010) \\
{[\mathrm{MW}]}\end{array}$ & $\begin{array}{c}\text { New } \\
\text { Capacity } \\
{[\mathrm{MW}]}\end{array}$ & $\begin{array}{c}\text { Existing } \\
(2010) \\
{[\mathrm{MW}]}\end{array}$ & $\begin{array}{c}\text { New } \\
\text { Capacity } \\
{[\text { MW] }}\end{array}$ \\
\hline Coal & 10,714 & 0 & 491 & 0 & 12,621 & 0 & 7,576 & 680 \\
ST & 1,597 & 0 & 12,906 & 50 & 800 & 0 & 239 & 0 \\
CC & 11,760 & 0 & 12,689 & 0 & 5,809 & 0 & 1,804 & 0 \\
CT & 2,875 & 0 & 4,613 & 0 & 1,731 & 0 & 2,034 & 0 \\
Nuclear & 3,875 & 0 & 4,550 & 0 & 1,146 & 0 & 0 & 0 \\
Pumped & & & & & & & & \\
Storage & 216 & 0 & 3,190 & 0 & 314 & 0 & 563 & 0 \\
Biomass & 29 & 35 & 889 & 714 & 427 & 717 & 8 & 162 \\
Geotherma & 0 & 252 & 2,182 & 156 & 435 & 781 & 0 & 0 \\
Hydro & 2,923 & 0 & 7,635 & 0 & 23,968 & 0 & 912 & 0 \\
Solar & 138 & 1,768 & 322 & 293 & 0 & 76 & 10 & 298 \\
Wind & 394 & 967 & 2,632 & 4187 & 5,067 & 2,364 & 2,597 & 5,753 \\
DG & 886 & 0 & 4,801 & 0 & 900 & 0 & 400 & 0 \\
\hline Total & $\mathbf{3 5 , 4 0 7}$ & $\mathbf{3 , 0 2 2}$ & $\mathbf{5 6 , 9 0 0}$ & $\mathbf{5 , 3 9 9}$ & $\mathbf{5 3 , 2 1 7}$ & $\mathbf{3 , 9 3 7}$ & $\mathbf{1 6 , 1 4 2}$ & $\mathbf{6 , 8 9 4}$ \\
\hline
\end{tabular}

Simulation results reveal that there are several congested paths in the system. The most congested path is the interface between Utah and the Los Angeles Department of Water and Power (LADWP). Several key congested paths, which experience numerous hours at their transfer limit for a month or more during the year, are shown in Figure 7.5 (shown with red stars) and tabulated in Table 7.2. 


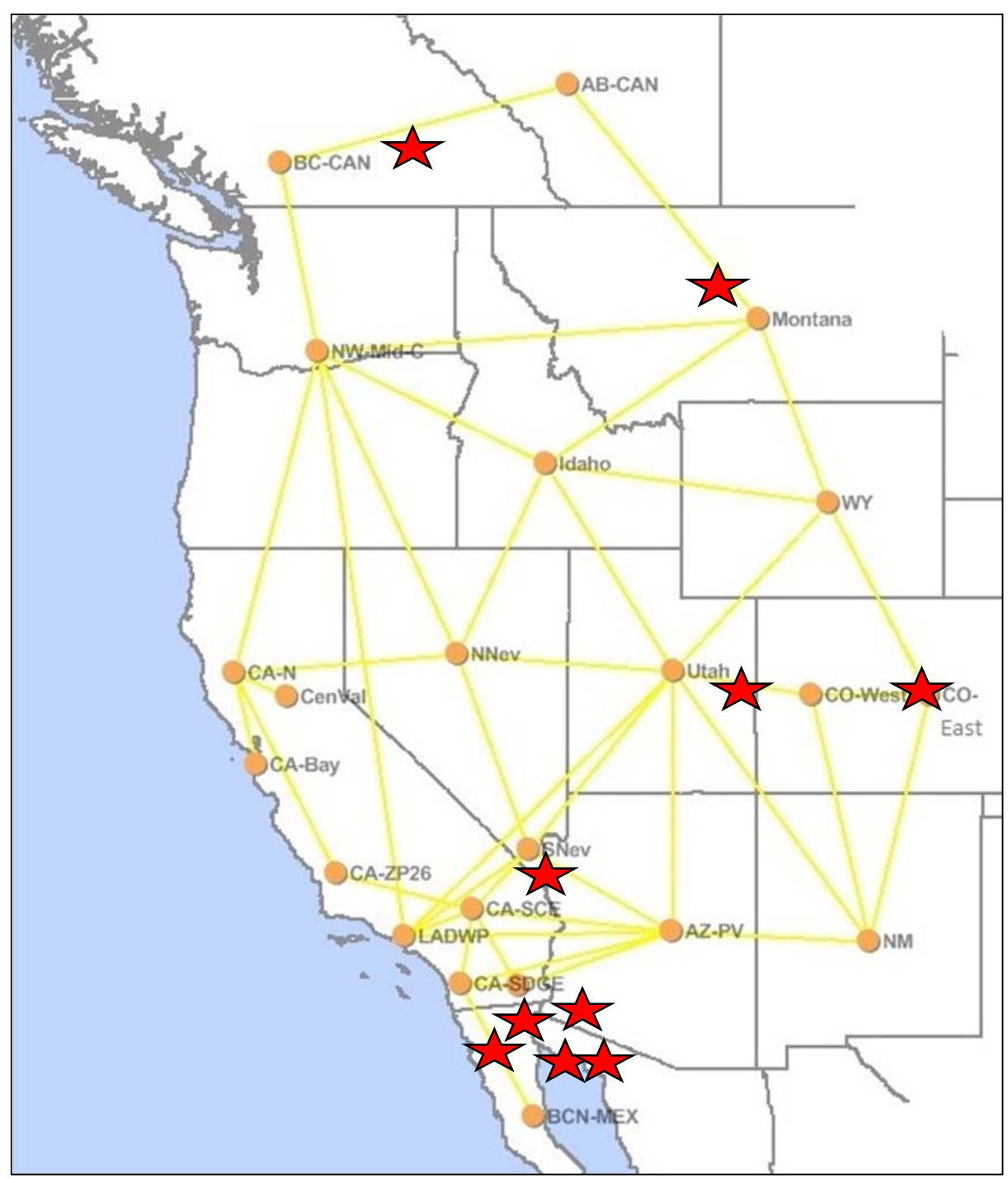

Figure 7.5. Key Congested Paths in WECC

Table 7.2. Number of Hours at $100 \%$ Transfer Limits

\begin{tabular}{lc}
\hline \multicolumn{1}{c}{ Interface } & $\begin{array}{c}\text { Without Storage } \\
{[\mathrm{h}]}\end{array}$ \\
\hline Alberta-BC (from Alberta to BC-CAN) & 2,363 \\
Alberta-Montana (from AB-CAN to Montana) & 2,600 \\
Arizona-Southern California Edison (from AZ-PV to SCE) & 4,527 \\
Arizona-San Diego Gas \&Electric (from AZ-PV to SDG\&E) & 4,071 \\
Arizona-Imperial Irrigation District (from Arizona to IID) & 751 \\
Arizona-Imperial Irrigation District (from IID to AZ-PV) & 1,104 \\
Arizona-Los Angeles (Arizona to LADWP) & 1,687 \\
CO-East-Wyoming (from CO-E to WY) & 906 \\
Imperial Irrig. Distr.-SCE (from IID to SCE) & 3,423 \\
Imperial Irrig. Distr.-SDG\&E (from IID to SDG\&E) & 3,760 \\
UTAH-LADWP (from Utah to LADWP) & 7,024 \\
UTAH-Wyoming (From WY to Utah) & 2,665 \\
\hline
\end{tabular}


Energy storage sizes and locations are needed to determine how to mitigate those congested paths. Energy storage locations are assumed to be at the sink ends of the paths, and sizes are determined using the assumptions outlined below.

Assume power flow on a path has $n$ hours at its limit during the year. In this case, the average number of hours in a day that path is congested is equal to $24^{*} n / 8,784$ (note that 2020 is a leap year that has 8,784 hours). To mitigate the path congestion in those hours, we propose storage with energy to supply an arbitrary fraction of 1/3 of the energy in that period, as shown in Figure 7.6. The apportioning of $1 / 3$ of the peak energy was somewhat arbitrary, guided by the intent to reduce the congestion significantly. Hence, the storage size is $(24 * n / 8784) *\left(F_{\max } / 3\right)$, where $F_{\max }$ is the path limit. We further assume that the storage energy (in MWh) determined as above has 10 hours of energy. The storage capacity (in MW) is therefore $1 / 10^{\text {th }}$ of its energy. The same approach is applied to determine the size of storage required to alleviate all congested paths. The total storage size for the system is called $\mathrm{x}$.

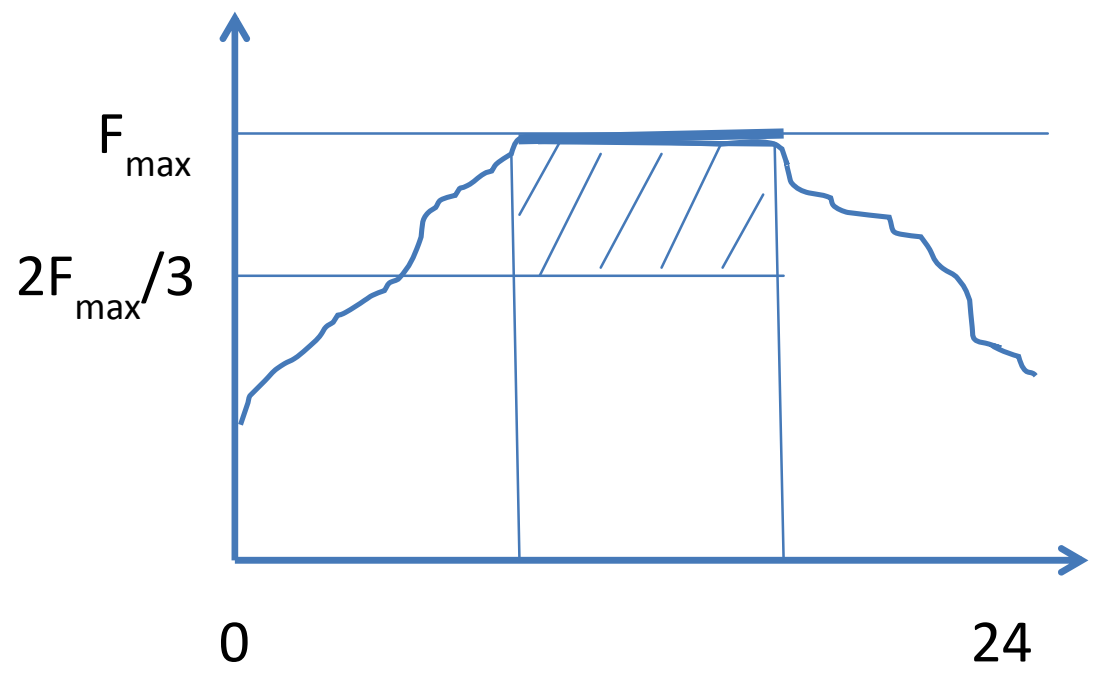

Figure 7.6. Determination of Storage Size

By applying the proposed approach, a total storage size needed is estimated as $\mathrm{x}=48,560 \mathrm{MWh}$ in which 42,710 MWh located in CAMX and 5,850 MWh located in NWPP. We do not consider applying energy storage for the path Alberta-BC because it is in Canadian territory. To determine the optimal storage size implemented at the two regions above, storage with sizes of $0.05 \times, 0.1 \times, 0.2 \times, 0.25 \times, 0.5 \times$, $0.75 \times, 1 \times, 1.25 \times$, and $2 \times$ was applied. The storage size is optimal when it yields the highest system profit. The profit at each region is calculated as the difference between revenue obtained from selling energy to the system when discharging the storage and cost incurred from buying energy from the system to charge the storage.

$$
\text { Profit }=\sum_{\text {All hours }}\left(G_{i}-L_{i}\right) L M P_{i}
$$

where $G_{i}$ is the energy generated by discharging the storage in hour $i, L_{i}$ is the energy consumed by charging the storage in hour $i$, and $L M P_{i}$ is the LMP in hour $i$ at location where storage is installed.

The system profit is defined as the summation of profit from all regions. 


\subsection{Arbitrage Results}

Using the approach outlined in the previous section, simulations were carried out for different energy storage sizes for the NWPP and CAMX. The revenue computed for each energy storage capacity includes the impact of the O\&M costs for energy storage and a capital recovery factor built in for existing and forecast power plants. The results of each simulation are presented in Table 7.3 and Figure 7.7. Revenues for the entire WECC grow from $\$ 4.3$ million annually at $243 \mathrm{MW}$ of installed capacity to $\$ 99.1$ million at 9,712 MW. As noted previously, of the 48,560 MWh of installed energy storage in the $1 \times$ scenario, 5,850 MWh is installed in the NWPP while the remaining 42,710 MWh would be installed in the CAMX.

Table 7.3. Annual Arbitrage Revenues in NWPP, CAMX, and WECC by Energy Storage Capacity (\$Thousands)

\begin{tabular}{rr|rrr}
\hline \multicolumn{2}{c|}{ Storage Size } & \multicolumn{3}{c}{ Annual Revenues in \$1000 } \\
\hline MWh & MW & NWPP & CAMX & WECC \\
\hline 2,400 & 240 & $\$ 433.9$ & $\$ 3,877.6$ & $\$ 4,311.5$ \\
4,900 & 490 & $\$ 866.4$ & $\$ 7,712.9$ & $\$ 8,579.4$ \\
9,700 & 970 & $\$ 1,710.2$ & $\$ 14,972.3$ & $\$ 16,682.5$ \\
12,100 & 1,210 & $\$ 2,120.1$ & $\$ 18,425.8$ & $\$ 20,545.8$ \\
24,300 & 2,430 & $\$ 4,117.1$ & $\$ 33,798.0$ & $\$ 37,915.1$ \\
36,400 & 3,640 & $\$ 5,968.4$ & $\$ 46,361.5$ & $\$ 52,329.9$ \\
48,600 & 4,860 & $\$ 7,627.9$ & $\$ 58,367.8$ & $\$ 65,995.6$ \\
60,700 & 6,070 & $\$ 8,835.6$ & $\$ 66,402.3$ & $\$ 75,237.9$ \\
97,100 & 9,710 & $\$ 11,810.7$ & $\$ 87,323.7$ & $\$ 99,134.3$ \\
\hline
\end{tabular}

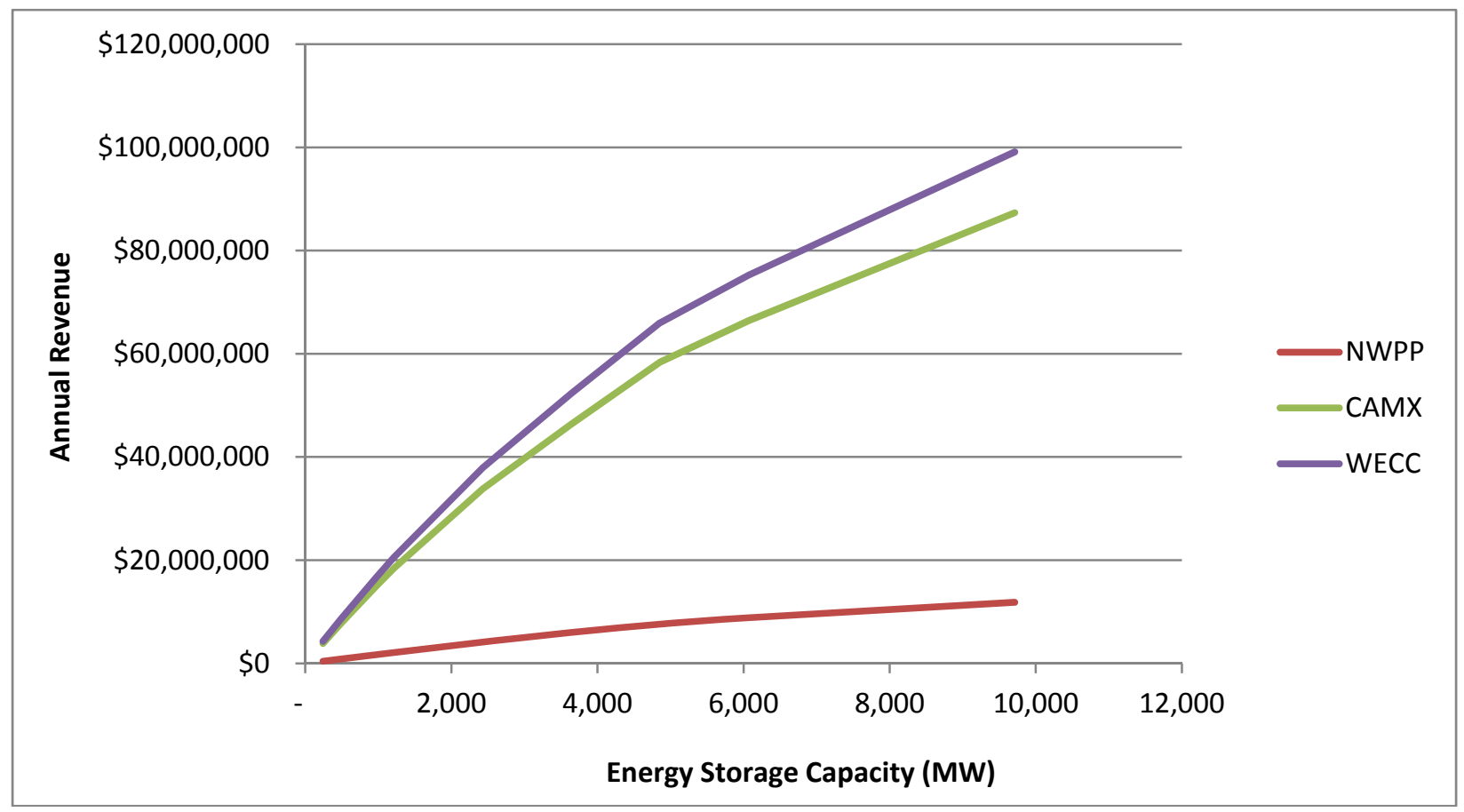

Figure 7.7. Arbitrage Revenues in NWPP, CAMX, and WECC per Year 
While annual revenue climbs as the capacity of energy storage increases, it does so at a declining rate of profitability. Table 7.4 and Figure 7.8 compare total and marginal revenue per MW per year for each of the energy storage capacities considered in this arbitrage analysis. Total revenue per MW is calculated by dividing total revenues obtained for a specific scenario by the corresponding storage size. Marginal revenue per MW is calculated by dividing the marginal revenues obtained by upsizing the storage capacity by the additional capacity added in the scenario. At $486 \mathrm{MW}$ of energy storage, annual revenues are estimated at \$8.6 million in the WECC or \$54.9 thousand per MW. At 4,856 MW of energy storage, annual revenues are estimated to grow to $\$ 66.0$ million in the WECC but revenues per MW fall to $\$ 42.0$ thousand per MW. Note that the marginal revenue curve presented in Figure 7.8 conforms to the theoretical curve presented in Figure 7.1b.

Table 7.4. Total and Marginal Arbitrage Revenues in WECC (\$Thousands per MW per year)

\begin{tabular}{cccc}
\hline \multicolumn{2}{c}{ Storage Size } & $\begin{array}{c}\text { Revenue in } \\
\text { \$1000 per MW } \\
\text { per Year }\end{array}$ & $\begin{array}{c}\text { Marginal Revenue } \\
\text { in } \$ 1000 \text { per MW } \\
\text { per Year }\end{array}$ \\
\hline MWh & MW & $\$ 17,758$ & $\$ 17,758$ \\
4,900 & 240 & $\$ 17,668$ & $\$ 17,577$ \\
9,700 & 490 & $\$ 17,177$ & $\$ 16,687$ \\
12,100 & 1,210 & $\$ 16,924$ & $\$ 15,911$ \\
24,300 & 2,430 & $\$ 15,616$ & $\$ 14,307$ \\
36,400 & 3,640 & $\$ 14,368$ & $\$ 11,874$ \\
48,600 & 4,860 & $\$ 13,591$ & $\$ 11,257$ \\
60,700 & 6,070 & $\$ 12,395$ & $\$ 7,613$ \\
97,100 & 9,710 & $\$ 10,207$ & $\$ 6,561$ \\
\hline
\end{tabular}

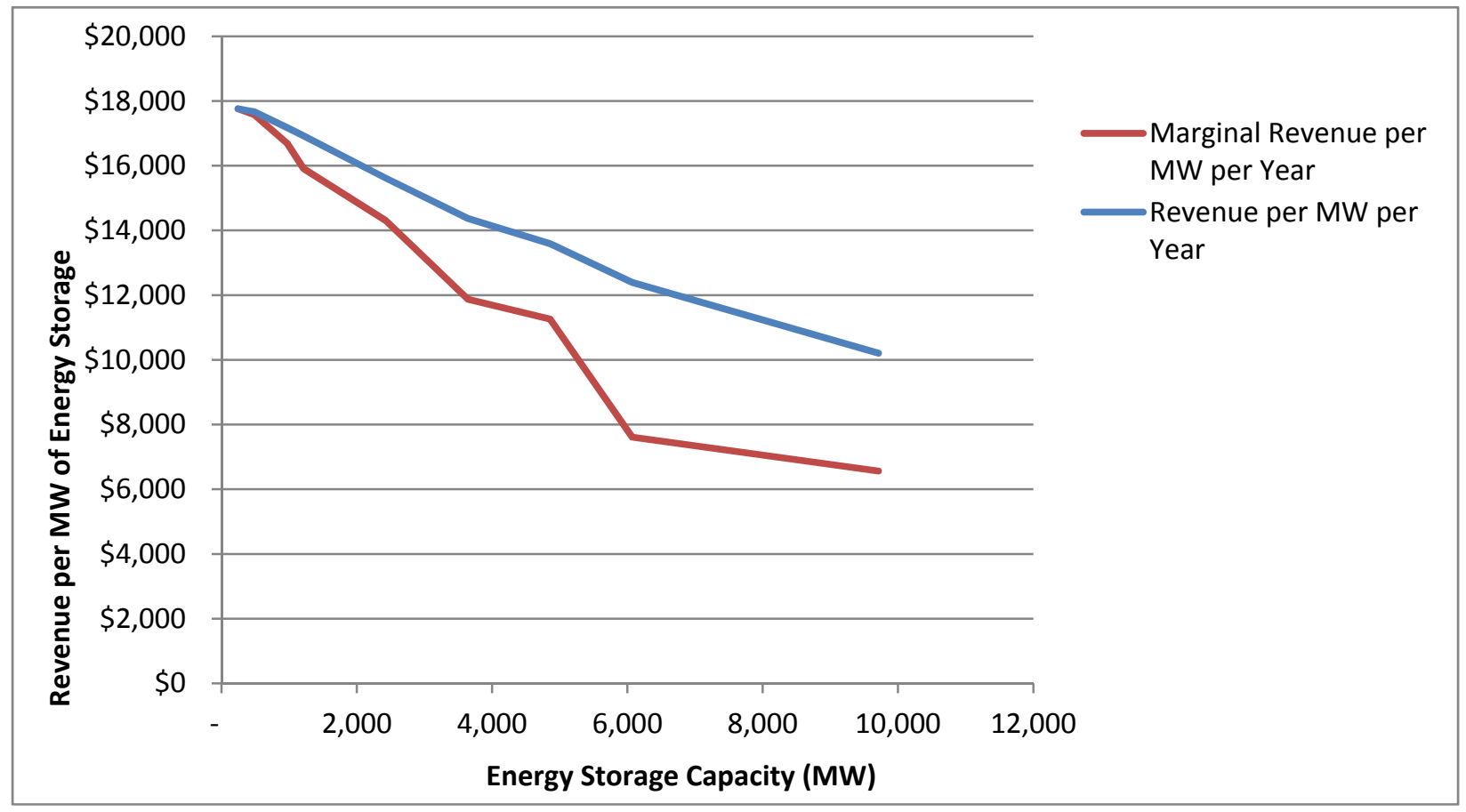

Figure 7.8. Total and Marginal Arbitrage Revenues in WECC per Year 
Adding a capacity value of $\$ 150-\mathrm{kW}$-year expands the values derived from energy storage significantly, as presented in Table 7.5 and Figure 7.9. For example, annual revenues generated by $243 \mathrm{MW}$ of storage would expand from $\$ 4.3$ million to $\$ 40.7$ million. Annual revenues for 9,712 MW of energy storage would increase from $\$ 99.1$ million to $\$ 1.6$ billion. In Figure 7.9 , note that the revenue figures demonstrate linearity when charted rather than concavity because the vast majority of the revenues would be derived from capacity values, as opposed to arbitrage, which are measured in fixed terms ( $\$ / \mathrm{kW}$-year). Thus, as the energy storage devices are scaled up, revenues grow largely proportionally when both capacity values and arbitrage service values are considered together.

Table 7.5. Annual Arbitrage and Capacity Reserve Revenues in NWPP, CAMX, and WECC by Energy Storage Capacity

\begin{tabular}{rrrrr}
\hline \multicolumn{2}{c}{ Storage Size } & \multicolumn{3}{c}{ Annual Revenues in $\$ 1000$} \\
\hline MWh & MW & \multicolumn{1}{c}{ NWPP } & \multicolumn{1}{c}{ CAMX } & \multicolumn{1}{c}{ WECC } \\
\hline 2,400 & 240 & $\$ 4,821.4$ & $\$ 35,910.1$ & $\$ 40,731.5$ \\
4,900 & 490 & $\$ 9,641.4$ & $\$ 71,777.9$ & $\$ 81,419.4$ \\
9,700 & 970 & $\$ 19,260.2$ & $\$ 143,102.3$ & $\$ 162,362.5$ \\
12,100 & 1,210 & $\$ 24,057.6$ & $\$ 178,588.3$ & $\$ 202,645.8$ \\
24,300 & 2,430 & $\$ 47,992.1$ & $\$ 354,123.0$ & $\$ 402,115.1$ \\
36,400 & 3,640 & $\$ 71,780.9$ & $\$ 526,849.0$ & $\$ 598,629.9$ \\
48,600 & 4,860 & $\$ 95,377.8$ & $\$ 699,017.8$ & $\$ 794,395.6$ \\
60,700 & 6,070 & $\$ 118,523.1$ & $\$ 867,214.8$ & $\$ 985,737.9$ \\
97,100 & 9,710 & $\$ 187,310.7$ & $\$ 1,368,623.7$ & $\$ 1,555,934.3$ \\
\hline
\end{tabular}

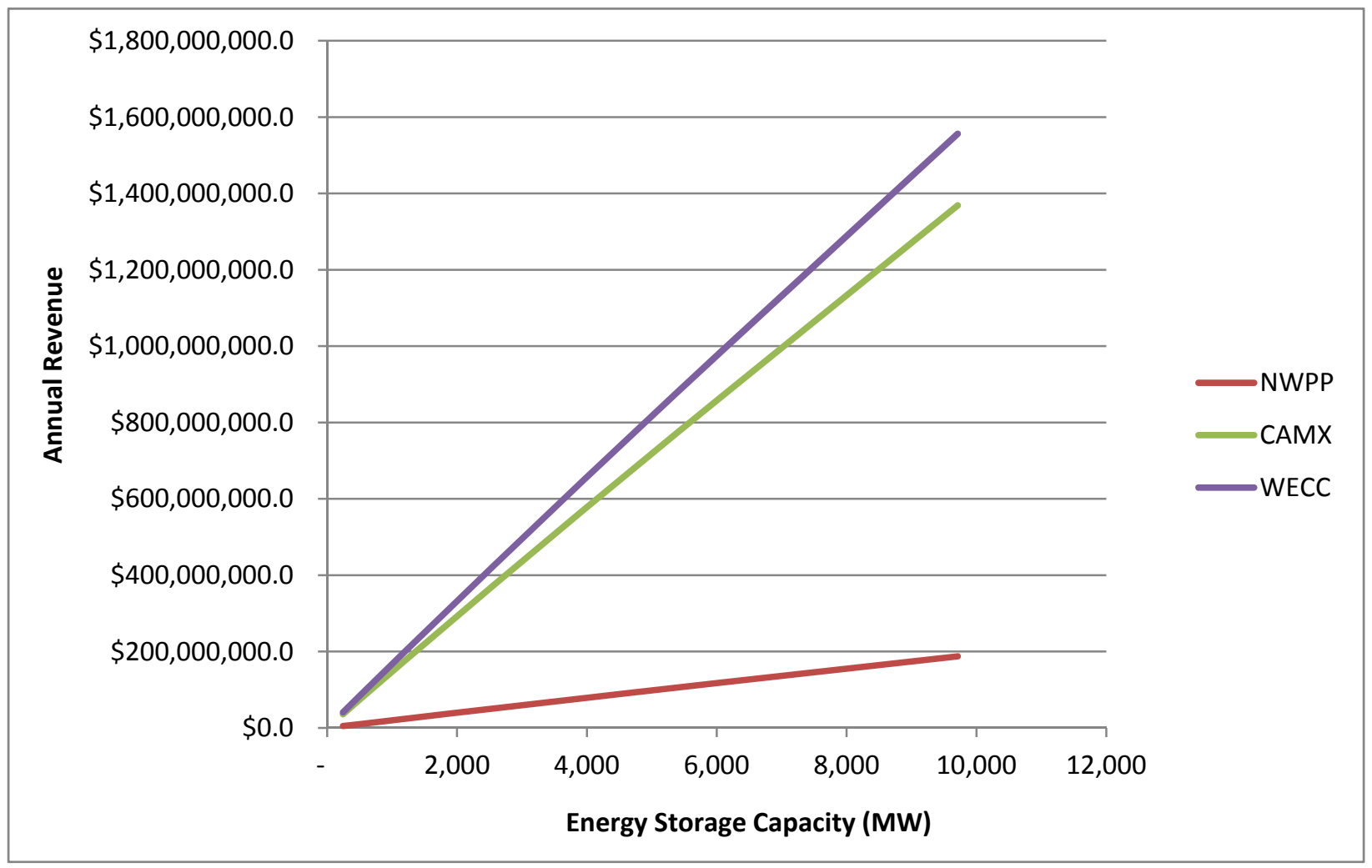

Figure 7.9. Arbitrage Revenues plus Capacity Values in NWPP, CAMX, and WECC per Year 
To determine the profitability of Na-S, Li-ion, and PH storage, the capital cost values presented in Table 3.2 were applied to the power (MW) and energy requirements (MWh) established for each increment of energy storage capacity considered for the NWPP, CAMX and WECC. For PH storage, capital costs are estimated at \$1,890 per MW and \$10 per MWh. Na-S capital costs are estimated at \$290 per MWh and \$200 per MW for BOP and PCS costs. Li-ion capital costs are estimated at \$510 per MWh and $\$ 200$ per MW for BOP and PCS costs.

Annualized capital costs for each energy storage option and each increment of capacity considered in this analysis are presented in Table 7.6. Annualized costs were constructed using the approach outlined in Section 5. To annualize capital costs, a fixed charge rate was constructed as a function of the system operating lifetime (10, 13, and 50 years for Li-ion, Na-S, and PH storage, respectively), after tax weighted cost of capital (8\%), income tax rate (40\%), and other taxes and insurance costs (2\%). These and other financial parameters are contained in Table 5.1.

Annual profits are presented in Table 7.7, demonstrating that arbitrage revenue expectations fall short of the revenue requirements necessary for cost recovery. This general finding applies to each scenario considered in this analysis. It would take either a significant increase in the peak- to off-peak LMP differential for extended periods of time or reductions in energy storage capital costs for energy arbitrage as designed in this analysis to break even. Under the current projections for the WECC, even the 243 MW scenario fails to yield profits when applied to the most profitable hours of the year. This result supports the conclusion that at a 30\% reserve margin, the WECC region is not sufficiently congested for energy storage to become cost-effective when used to provide only arbitrage services.

Table 7.8 presents the annual energy storage profits in the WECC when a capacity value is added to arbitrage values. While significant, the credits are insufficient to close the revenue shortfall. The findings of this analysis indicate that pumped hydro capital costs would need to drop to \$1,500 per MW to break even while Na-S and Li-ion costs would need to drop to $\$ 90$ per MWh. Given that we forecast $\mathrm{Na}-\mathrm{S}$ and Li-ion capital costs in 2020 at \$290 and \$510 per kWh, respectively, cost reductions of 69\% (Na-S) and $82 \%$ (Li-ion) would be required to break even. Pumped hydro capital costs would need to drop by $21 \%$ to break even.

While the findings of this analysis suggest that profits from energy arbitrage and capacity value are insufficient to achieve capital cost recovery, it is important to note that there are several other services that could be supplied by energy storage technologies that were excluded from this assessment. These services include load following, transmission and distribution upgrade deferral, power quality enhancements, and electricity service reliability. Thus, revenues from the energy storage technologies are not fully realized due to the limited focus of the analysis. Additional research is therefore necessary to examine the full revenue potential of energy storage used in multiple applications. 
Table 7.6. Annualized Capital Costs for Na-S Batteries, Li-Ion Batteries, and Pumped Hydro

\begin{tabular}{|c|c|c|c|c|c|}
\hline \multicolumn{3}{|c|}{ Storage Size } & \multicolumn{3}{|c|}{ Annualized Costs } \\
\hline MWh & MW & $\mathrm{X}^{(\mathrm{a})}$ & Pumped Hydro & $\mathrm{Na}-\mathrm{S}$ & Li-Ion \\
\hline \multicolumn{6}{|l|}{$N W P P$} \\
\hline 293 & 29 & 0.05 & $\$ 5,937,165$ & $\$ 13,329,225$ & $\$ 26,199,225$ \\
\hline 585 & 59 & 0.10 & $\$ 11,874,330$ & $\$ 26,658,450$ & $\$ 52,398,450$ \\
\hline 1,170 & 117 & 0.20 & $\$ 23,748,660$ & $\$ 53,316,900$ & $\$ 104,796,900$ \\
\hline 1,463 & 146 & 0.25 & $\$ 29,685,825$ & $\$ 66,646,125$ & $\$ 130,996,125$ \\
\hline 2,925 & 293 & 0.50 & $\$ 59,371,650$ & $\$ 133,292,250$ & $\$ 261,992,250$ \\
\hline 4,388 & 439 & 0.75 & $\$ 89,057,475$ & $\$ 199,938,375$ & $\$ 392,988,375$ \\
\hline 5,850 & 585 & 1.00 & $\$ 118,743,300$ & $\$ 266,584,500$ & $\$ 523,984,500$ \\
\hline 7,313 & 731 & 1.25 & $\$ 148,429,125$ & $\$ 333,230,625$ & $\$ 654,980,625$ \\
\hline 11,700 & 1,170 & 2.00 & $\$ 237,486,600$ & $\$ 533,169,000$ & $\$ 1,047,969,000$ \\
\hline \multicolumn{6}{|l|}{$C A M X$} \\
\hline 2,136 & 214 & 0.05 & $\$ 43,346,379$ & $\$ 97,314,735$ & $\$ 191,276,735$ \\
\hline 4,271 & 427 & 0.10 & $\$ 86,692,758$ & $\$ 194,629,470$ & $\$ 382,553,470$ \\
\hline 8,542 & 854 & 0.20 & $\$ 173,385,516$ & $\$ 389,258,940$ & $\$ 765,106,940$ \\
\hline 10,678 & 1,068 & 0.25 & $\$ 216,731,895$ & $\$ 486,573,675$ & $\$ 956,383,675$ \\
\hline 21,355 & 2,136 & 0.50 & $\$ 433,463,790$ & $\$ 973,147,350$ & $\$ 1,912,767,350$ \\
\hline 32,033 & 3,203 & 0.75 & $\$ 650,195,685$ & $\$ 1,459,721,025$ & $\$ 2,869,151,025$ \\
\hline 42,710 & 4,271 & 1.00 & $\$ 866,927,580$ & $\$ 1,946,294,700$ & $\$ 3,825,534,700$ \\
\hline 53,388 & 5,339 & 1.25 & $\$ 1,083,659,475$ & $\$ 2,432,868,375$ & $\$ 4,781,918,375$ \\
\hline 85,420 & 8,542 & 2.00 & $\$ 1,733,855,160$ & $\$ 3,892,589,400$ & $\$ 7,651,069,400$ \\
\hline \multicolumn{6}{|l|}{ WECC } \\
\hline 2,428 & 243 & 0.05 & $\$ 49,283,544$ & $\$ 110,643,960$ & $\$ 217,475,960$ \\
\hline 4,856 & 486 & 0.10 & $\$ 98,567,088$ & $\$ 221,287,920$ & $\$ 434,951,920$ \\
\hline 9,712 & 971 & 0.20 & $\$ 197,134,176$ & $\$ 442,575,840$ & $\$ 869,903,840$ \\
\hline 12,140 & 1,214 & 0.25 & $\$ 246,417,720$ & $\$ 553,219,800$ & $\$ 1,087,379,800$ \\
\hline 24,280 & 2,428 & 0.50 & $\$ 492,835,440$ & $\$ 1,106,439,600$ & $\$ 2,174,759,600$ \\
\hline 36,420 & 3,642 & 0.75 & $\$ 739,253,160$ & $\$ 1,659,659,400$ & $\$ 3,262,139,400$ \\
\hline 48,560 & 4,856 & 1.00 & $\$ 985,670,880$ & $\$ 2,212,879,200$ & $\$ 4,349,519,200$ \\
\hline 60,700 & 6,070 & 1.25 & $\$ 1,232,088,600$ & $\$ 2,766,099,000$ & $\$ 5,436,899,000$ \\
\hline 97,120 & 9,712 & 2.00 & $\$ 1,971,341,760$ & $\$ 4,425,758,400$ & $\$ 8,699,038,400$ \\
\hline
\end{tabular}

(a) A multiplier of an arbitrary reference capacity in MW. The reference capacity is different in each WECC sub-region. 
Table 7.7. Annualized Profits for Na-S Batteries, Li-Ion Batteries, and Pumped Hydro (Capacity Value Excluded)

\begin{tabular}{|c|c|c|c|c|c|}
\hline \multicolumn{3}{|c|}{ Storage Size } & \multicolumn{3}{|c|}{ Annualized Profits } \\
\hline MWh & MW & $\mathrm{x}$ & Pumped Hydro & $\mathrm{Na}-\mathrm{S}$ & Li-Ion \\
\hline \multicolumn{6}{|l|}{$N W P P$} \\
\hline 293 & 29 & 0.05 & $-\$ 5,503,218$ & $-\$ 12,895,278$ & $-\$ 25,765,278$ \\
\hline 585 & 59 & 0.10 & $-\$ 11,007,906$ & $-\$ 25,792,026$ & $-\$ 51,532,026$ \\
\hline 1,170 & 117 & 0.20 & $-\$ 22,038,445$ & $-\$ 51,606,685$ & $-\$ 103,086,685$ \\
\hline 1,463 & 146 & 0.25 & $-\$ 27,565,774$ & $-\$ 64,526,074$ & $-\$ 128,876,074$ \\
\hline 2,925 & 293 & 0.50 & $-\$ 55,254,527$ & $-\$ 129,175,127$ & $-\$ 257,875,127$ \\
\hline 4,388 & 439 & 0.75 & $-\$ 83,089,050$ & $-\$ 193,969,950$ & $-\$ 387,019,950$ \\
\hline 5,850 & 585 & 1.00 & $-\$ 111,115,450$ & $-\$ 258,956,650$ & $-\$ 516,356,650$ \\
\hline 7,313 & 731 & 1.25 & $-\$ 139,593,562$ & $-\$ 324,395,062$ & $-\$ 646,145,062$ \\
\hline 11,700 & 1,170 & 2.00 & $-\$ 225,675,906$ & $-\$ 521,358,306$ & $-\$ 1,036,158,306$ \\
\hline \multicolumn{6}{|l|}{ CAMX } \\
\hline 2,136 & 214 & 0.05 & $-\$ 39,468,787$ & $-\$ 93,437,143$ & $-\$ 187,399,143$ \\
\hline 4,271 & 427 & 0.10 & $-\$ 78,979,827$ & $-\$ 186,916,539$ & $-\$ 374,840,539$ \\
\hline 8,542 & 854 & 0.20 & $-\$ 158,413,231$ & $-\$ 374,286,655$ & $-\$ 750,134,655$ \\
\hline 10,678 & 1,068 & 0.25 & $-\$ 198,306,144$ & $-\$ 468,147,924$ & $-\$ 937,957,924$ \\
\hline 21,355 & 2,136 & 0.50 & -\$399,665,839 & $-\$ 939,349,399$ & $-\$ 1,878,969,399$ \\
\hline 32,033 & 3,203 & 0.75 & $-\$ 603,834,211$ & $-\$ 1,413,359,551$ & $-\$ 2,822,789,551$ \\
\hline 42,710 & 4,271 & 1.00 & $-\$ 808,559,828$ & -\$1,887,926,948 & $-\$ 3,767,166,948$ \\
\hline 53,388 & 5,339 & 1.25 & $-\$ 1,017,257,163$ & $-\$ 2,366,466,063$ & $-\$ 4,715,516,063$ \\
\hline 85,420 & 8,542 & 2.00 & $-\$ 1,646,531,507$ & $-\$ 3,805,265,747$ & $-\$ 7,563,745,747$ \\
\hline \multicolumn{6}{|l|}{ WECC } \\
\hline 2,428 & 243 & 0.05 & $-\$ 44,972,005$ & $-\$ 106,332,421$ & $-\$ 213,164,421$ \\
\hline 4,856 & 486 & 0.10 & $-\$ 89,987,734$ & $-\$ 212,708,566$ & $-\$ 426,372,566$ \\
\hline 9,712 & 971 & 0.20 & $-\$ 180,451,677$ & $-\$ 425,893,341$ & $-\$ 853,221,341$ \\
\hline 12,140 & 1,214 & 0.25 & $-\$ 225,871,918$ & $-\$ 532,673,998$ & $-\$ 1,066,833,998$ \\
\hline 24,280 & 2,428 & 0.50 & $-\$ 454,920,365$ & $-\$ 1,068,524,525$ & $-\$ 2,136,844,525$ \\
\hline 36,420 & 3,642 & 0.75 & $-\$ 686,923,261$ & $-\$ 1,607,329,501$ & $-\$ 3,209,809,501$ \\
\hline 48,560 & 4,856 & 1.00 & -\$919,675,278 & $-\$ 2,146,883,598$ & $-\$ 4,283,523,598$ \\
\hline 60,700 & 6,070 & 1.25 & $-\$ 1,156,850,725$ & $-\$ 2,690,861,125$ & $-\$ 5,361,661,125$ \\
\hline 97,120 & 9,712 & 2.00 & $-\$ 1,872,207,413$ & $-\$ 4,326,624,053$ & $-\$ 8,599,904,053$ \\
\hline
\end{tabular}


Table 7.8. Annualized Profits for Na-S Batteries, Li-Ion Batteries, and Pumped Hydro in WECC with Capacity Value and Arbitrage Values

\begin{tabular}{rrrrrr}
\hline & Storage Size & \multicolumn{3}{c}{ Annualized Profits } \\
\hline \multicolumn{1}{c}{ MWh } & MW & $\mathrm{x}$ & Pumped Hydro & Na-S & \multicolumn{1}{c}{ Li-Ion } \\
\hline 2,428 & 243 & 0.05 & $-\$ 8,552,005$ & $-\$ 69,912,421$ & $-\$ 176,744,421$ \\
4,856 & 486 & 0.10 & $-\$ 17,147,734$ & $-\$ 139,868,566$ & $-\$ 353,532,566$ \\
9,712 & 971 & 0.20 & $-\$ 34,771,677$ & $-\$ 280,213,341$ & $-\$ 707,541,341$ \\
12,140 & 1,214 & 0.25 & $-\$ 43,771,918$ & $-\$ 350,573,998$ & $-\$ 884,733,998$ \\
24,280 & 2,428 & 0.50 & $-\$ 90,720,365$ & $-\$ 704,324,525$ & $-\$ 1,772,644,525$ \\
36,420 & 3,642 & 0.75 & $-\$ 140,623,261$ & $-\$ 1,061,029,501$ & $-\$ 2,663,509,501$ \\
48,560 & 4,856 & 1.00 & $-\$ 191,275,278$ & $-\$ 1,418,483,598$ & $-\$ 3,555,123,598$ \\
60,700 & 6,070 & 1.25 & $-\$ 246,350,725$ & $-\$ 1,780,361,125$ & $-\$ 4,451,161,125$ \\
97,120 & 9,712 & 2.00 & $-\$ 415,407,413$ & $-\$ 2,869,824,053$ & $-\$ 7,143,104,053$ \\
\hline
\end{tabular}

\subsection{Final Observations on Arbitrage Results}

The arbitrage analysis was performed using a production cost model with a zonal representation of the WECC. The zonal representation of a load zone assumes no congestion within the zone. Congestion can only occur between zones by reaching inter-zonal transfer limits. It is assumed that if a nodal representation of the transmission system were used that represents all major transmission lines with ratings of $139 \mathrm{kV}$ lines and above, that more small niche markets of high congestions may have been identified. This may raise the economic viability of energy arbitrage, however, the total market size (size of the storage to mitigate congestion) would most likely be small. 


\subsection{Results: Balancing Requirements}

This section presents the results of the analysis by 4 WECC sub-regions. First, balancing requirements results are summarized for all the sub-regions. Then, the balancing requirements results, the size of energy storage needed to provide the balancing services, the LCC analysis results of different energy storage technologies, and the results of using energy storage for energy arbitrage service are presented for every sub-region.

\subsection{Total Balancing Requirements WECC}

The total balancing requirements for every sub-region using the BPA's 99.5\% probability bound and based on 1-year simulation results are shown in Table 8.1. The main factors that affect the balancing requirements are the wind adoption level, wind forecast accuracy, load forecast accuracy, peak demand level of every region, load intra-hour variations and wind intra-hour variations in every region. NWPP and CAMX has high wind adoption level and high peak demand, so the power balancing requirements are high. If we compare the power balancing requirements of AZNM and RMPA, AZNM has higher power requirements. But AZNM has much less wind adoption than RMPA. The main reason is that AZNM peak load is almost 2.5 times of peak load of RMPA. The balancing requirements are separated based on the causation of the requirements and reported in Table 8.3 and Table 8.4. AZNM and CAMX balancing needs are mainly caused by load uncertainty while NWPP and RMPA balancing needs are evenly caused by load and wind. The balancing power requirements caused by wind capacity are around $20 \%-30 \%$ of the installed wind capacity in each sub-region. Though the load forecast is much more precise than wind forecast, because the peak demands will reach $33 \mathrm{GW}$ in AZNM, $68 \mathrm{GW}$ in CAMX, $71 \mathrm{GW}$ in NWPP, and $14 \mathrm{GW}$ in RMPA, significant amount of balancing reserve is needed to accommodate the uncertainty of load.

The results indicate that the total balancing requirements span a spectrum of frequencies, from minute-to-minute variability (intra-hour balancing) to those indicating cycles over several hours (interhour balancing). This analysis focused on the intra-hour balancing market because the sharp ramp rates in this market are of significant concern to grid operators. The intra-hour balancing requirements were decomposed from the total balancing requirements and are presented in Table 8.2. Comparing Table 8.2 and Table 7.1, the power capacity requirements are significantly reduced in the intra-hour balancing, but the ramp rate requirements are very well preserved.

Table 8.1. Total Balancing Requirements for every NERC Region in WECC

\begin{tabular}{lcccc}
\hline & $\begin{array}{c}\text { Power } \\
(\mathrm{MW})\end{array}$ & $\begin{array}{c}\text { Balancing Power Required as } \\
\text { a Percentage of Average } \\
\text { Demand (\%) }\end{array}$ & $\begin{array}{c}\text { Balancing Power Required } \\
\text { as a Percentage of Peak } \\
\text { Demand (\%) }\end{array}$ & $\begin{array}{c}\text { Ramp Rate } \\
\text { (MW/min) }\end{array}$ \\
\hline AZNM & 2740 & 20 & 11 & 350 \\
& -3550 & 11 & 6 & 220 \\
CAMX & 4130 & & 5 & 190 \\
& -2920 & 7 & 15 & 90 \\
NWPP & 3430 & 24 & & \\
& -2730 & & & \\
RMPA & 2150 & & & \\
\end{tabular}


Table 8.2. Intra-hour Balancing Requirements for NERC Sub-Regions in WECC.

\begin{tabular}{|c|c|c|c|c|}
\hline & $\begin{array}{l}\text { Power } \\
\text { (MW) }\end{array}$ & $\begin{array}{c}\text { Balancing Power Required as } \\
\text { a Percentage of Average } \\
\text { Demand (\%) }\end{array}$ & $\begin{array}{c}\text { Balancing Power Required } \\
\text { as a Percentage of Peak } \\
\text { Demand (\%) }\end{array}$ & $\begin{array}{l}\text { Ramp Rate } \\
\text { (MW/min) }\end{array}$ \\
\hline AZNM & $\begin{array}{r}1090 \\
-1220\end{array}$ & 11 & 11 & 290 \\
\hline CAMX & $\begin{array}{r}1790 \\
-2400\end{array}$ & 5 & 5 & 230 \\
\hline NWPP & $\begin{array}{r}1690 \\
-2020\end{array}$ & 4 & 4 & 200 \\
\hline RMPA & $\begin{array}{r}670 \\
-670\end{array}$ & 22 & 15 & 120 \\
\hline
\end{tabular}

Table 8.3. Total Balancing Requirements Caused by Wind Uncertainty

\begin{tabular}{lrcc}
\hline & $\begin{array}{c}\text { Power } \\
\text { (MW) }\end{array}$ & $\begin{array}{c}\text { Balancing Power Required as } \\
\text { a Percentage of Installed } \\
\text { Wind Capacity (\%) }\end{array}$ & $\begin{array}{c}\text { Ramp Rate } \\
\text { (MW/min) }\end{array}$ \\
\hline AZNM & 290 & 22 & 10 \\
CAMX & -290 & 22 & 60 \\
& 1460 & 28 & 60 \\
NWPP & -1260 & & 100 \\
& 2730 & 31 & \\
RMPA & -1650 & & \\
& 1980 & & \\
\hline
\end{tabular}

Table 8.4. Total Balancing Requirements Caused by Load Uncertainty

\begin{tabular}{cccc}
\hline & $\begin{array}{c}\text { Power } \\
(\mathrm{MW})\end{array}$ & $\begin{array}{c}\text { Balancing Power Required as } \\
\text { a Percentage of Peak Demand } \\
(\%)\end{array}$ & $\begin{array}{c}\text { Ramp Rate } \\
(\mathrm{MW} / \mathrm{min})\end{array}$ \\
\hline AZNM & 2730 & 11 & 350 \\
& -3520 & 6 & 210 \\
CAMX & 4370 & 4 & 180 \\
& -2710 & & 80 \\
NWPP & 2690 & 12 & \\
& -2550 & & \\
RMPA & 1680 & & \\
& -1450 & & \\
\hline
\end{tabular}

Table 8.4 shows the intra-hour balancing requirements caused by wind uncertainty only. Notice the difference between the last column of Table 8.4 and the third column of Table 8.2. A capacity of $8 \%$ to $15 \%$ of wind capacity is needed for intra-hour balancing, while $22 \%$ to $30 \%$ of wind capacity is needed for total balancing (intra + inter-hour).

Table 8.5 shows the intra-hour balancing requirements caused by load uncertainty only. Notice that the balancing requirements produced by load uncertainty are larger than those produced by wind uncertainty. 
Table 8.5. Balancing Requirements (Intra-hour) for NERC Sub-Regions in WECC caused by Wind Variability Only (without considering load variability)

\begin{tabular}{ccccc}
\hline \multicolumn{5}{c}{ Intra-Hour Balancing Power Requirements Caused by Wind Variability Only } \\
\hline & & $\begin{array}{c}\text { As a Percentage of } \\
\text { Average Demand (\%) }\end{array}$ & $\begin{array}{c}\text { As a Percentage of Peak } \\
\text { Demand (\%) }\end{array}$ & $\begin{array}{c}\text { As a Percentage of Installed } \\
\text { Wind Capacity (\%) }\end{array}$ \\
\hline AZNM & 170 & 1.0 & 0.5 & 12.8 \\
CAMX & 940 & 2.5 & 1.4 & 14.4 \\
NWPP & 1,070 & 2.1 & 1.5 & 11.0 \\
RMPA & 500 & 5.6 & 3.6 & 8.0 \\
\hline
\end{tabular}

Table 8.6. Balancing Requirements (Intra-hour) for NERC Sub-Regions in WECC caused by Load Variability Only (without considering wind variability)

\begin{tabular}{ccccc}
\hline \multicolumn{5}{c}{ Intra-Hour Balancing Power Requirements Caused by Load Variability Only } \\
\hline & \multicolumn{1}{c}{ As a Percentage of } & As a Percentage of Peak & As a Percentage of Installed \\
Derage Demand (\%) & Demand (\%) & Wind Capacity (\%) \\
\hline AZNM & 1132 & 6 & 3 & 83 \\
CAMX & 2809 & 7 & 4 & 43 \\
NWPP & 1772 & 4 & 2 & 18 \\
RMPA & 631 & 7 & 5 & 10 \\
\hline
\end{tabular}

\subsection{Additional Balancing Requirements for WECC}

The additional balancing requirements for every sub-region using the BPA's 99.5\% probability bound and based on one-year simulation results are shown in Table 8.1. The additional balancing requirements are wind uncertainty driven. That is, a region with more hypothetical wind capacity, the region has higher balancing requirements. Contrasted to the total balancing requirements, the additional balancing requirements are much lower. The main reasons are the incremental load is only $6 \%$ of the total load and the hypothetical wind is around $55 \%$ of the total wind capacity.

Table 8.7. Additional Balancing Requirements for every NERC Region in WECC

\begin{tabular}{|c|c|c|c|c|}
\hline & Power (MW) & $\begin{array}{c}\text { Balancing Power Required } \\
\text { as a Percentage of } \\
\text { Hypothetical Wind Capacity } \\
(\%)\end{array}$ & $\begin{array}{c}\text { Hypothetical Wind } \\
\text { Capacity (MW) }\end{array}$ & $\begin{array}{l}\text { Ramp Rate } \\
\text { (MW/min) }\end{array}$ \\
\hline$\overline{A Z N M}$ & $\begin{array}{r}330 \\
-450\end{array}$ & 46 & 970 & 20 \\
\hline CAMX & $\begin{array}{r}970 \\
-1000\end{array}$ & 24 & 4190 & 40 \\
\hline NWPP & $\begin{array}{r}720 \\
-740\end{array}$ & 15 & 5110 & 20 \\
\hline RMPA & $\begin{array}{r}1300 \\
-910\end{array}$ & 43 & 3010 & 50 \\
\hline
\end{tabular}




\subsubsection{Southwest (AZNM)}

The pattern of balancing signal determines the size of energy storage needed to provide the signal. Specifically, the magnitude of the signal determines the power capacity requirement of energy storage.

\subsubsection{Balancing Requirements}

Monthly and daily balancing signals of region AZNM are shown in Figure 8.1 and Figure 8.2, respectively. Long cycles across several days are included in the balancing signal. If only energy storage is used to meet this balancing signal, energy storage that has several days of energy capacity is needed. The long cycle energy storage is very expensive especially for emerging energy storage technologies such as batteries and flywheels. Furthermore, traditional generation resources should have sufficient ramp capability to meet these long cycles because the long cycles usually do not have steep slope. Based on the whole year simulation, the balancing power requirements are $2740 \mathrm{MW}$ of incremental (inc) capacity and 3554 MW of decremental (dec) capacity, using the BPA’s customary $99.5 \%$ probability bound. The balancing requirements for August, especially the incremental capacity, are lower than the annual requirements.

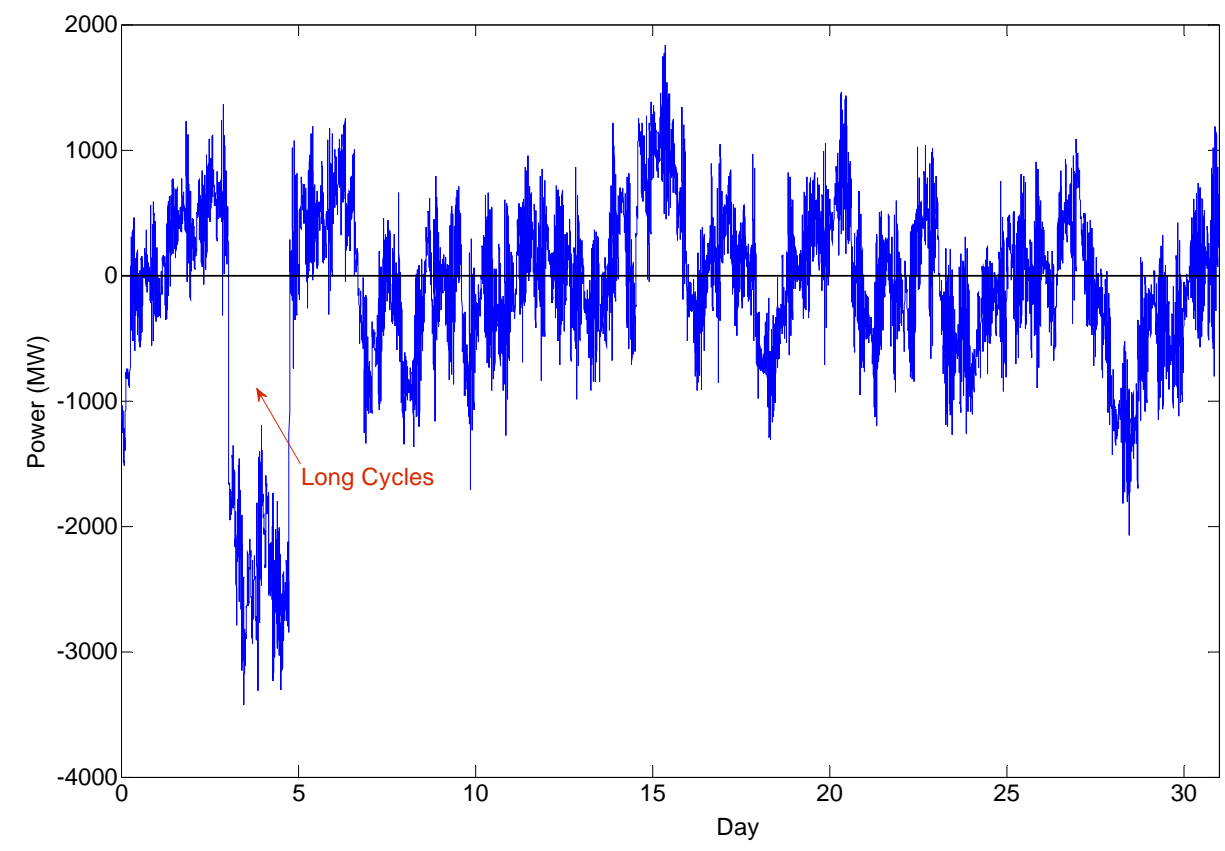

Figure 8.1. One Month Total Balancing Signal of August 2020 for AZNM 


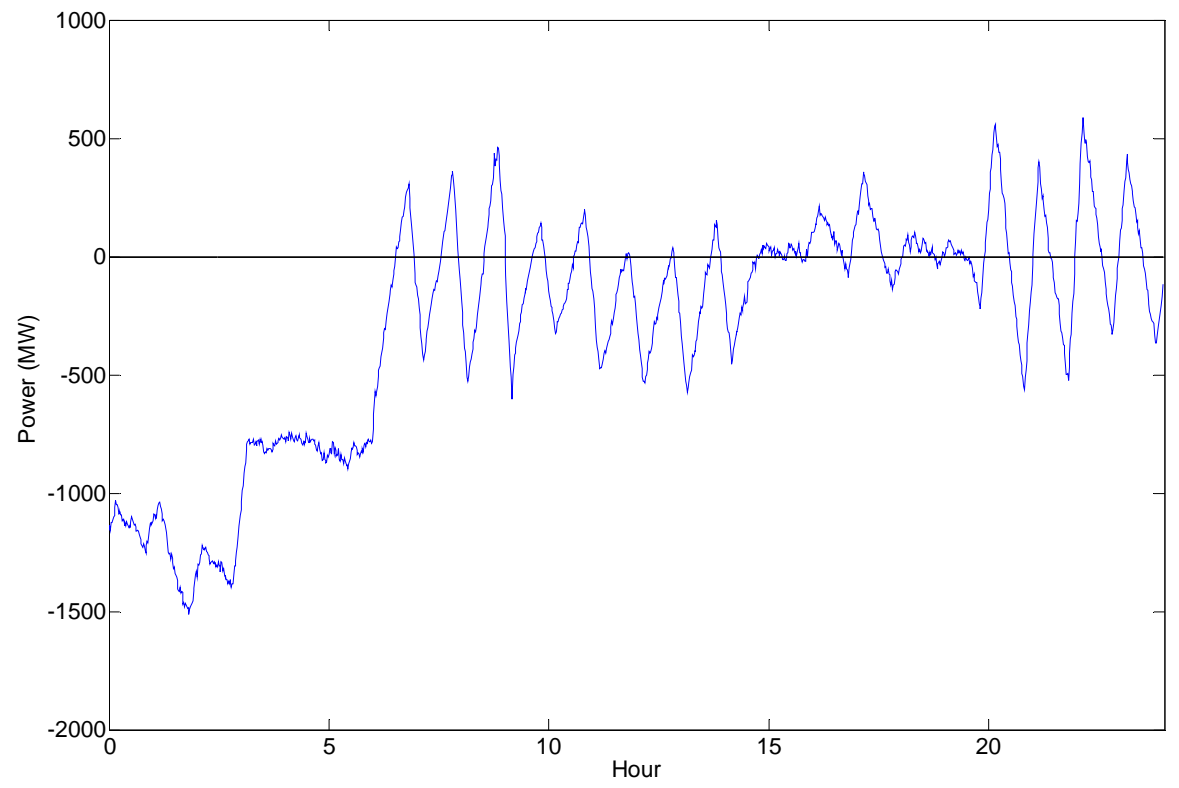

Figure 8.2. One Typical Day Total Balancing Signal of August 2020 for AZNM

Figure 8.3 shows balancing signals caused by load and caused by wind separately for the region AZNM for one month. For the southwest region, the balancing requirements are mainly caused by load uncertainty because the wind resource in the southwest region is scarce and the peak load level is high. Figure 8.4 presents the same balancing signals for one day.

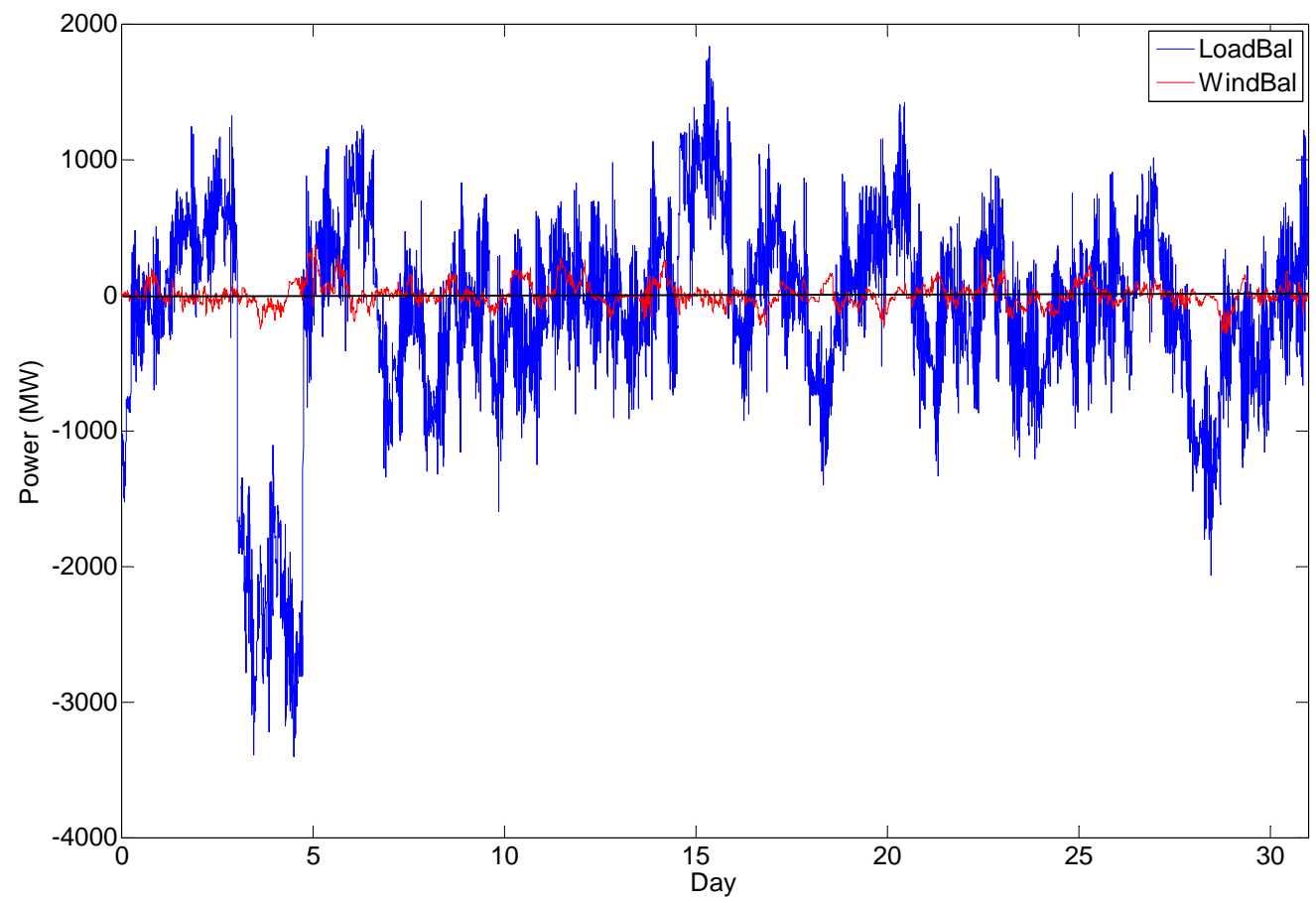

Figure 8.3. One Month Balancing Requirements Caused by Load and Wind Respectively for AZNM 


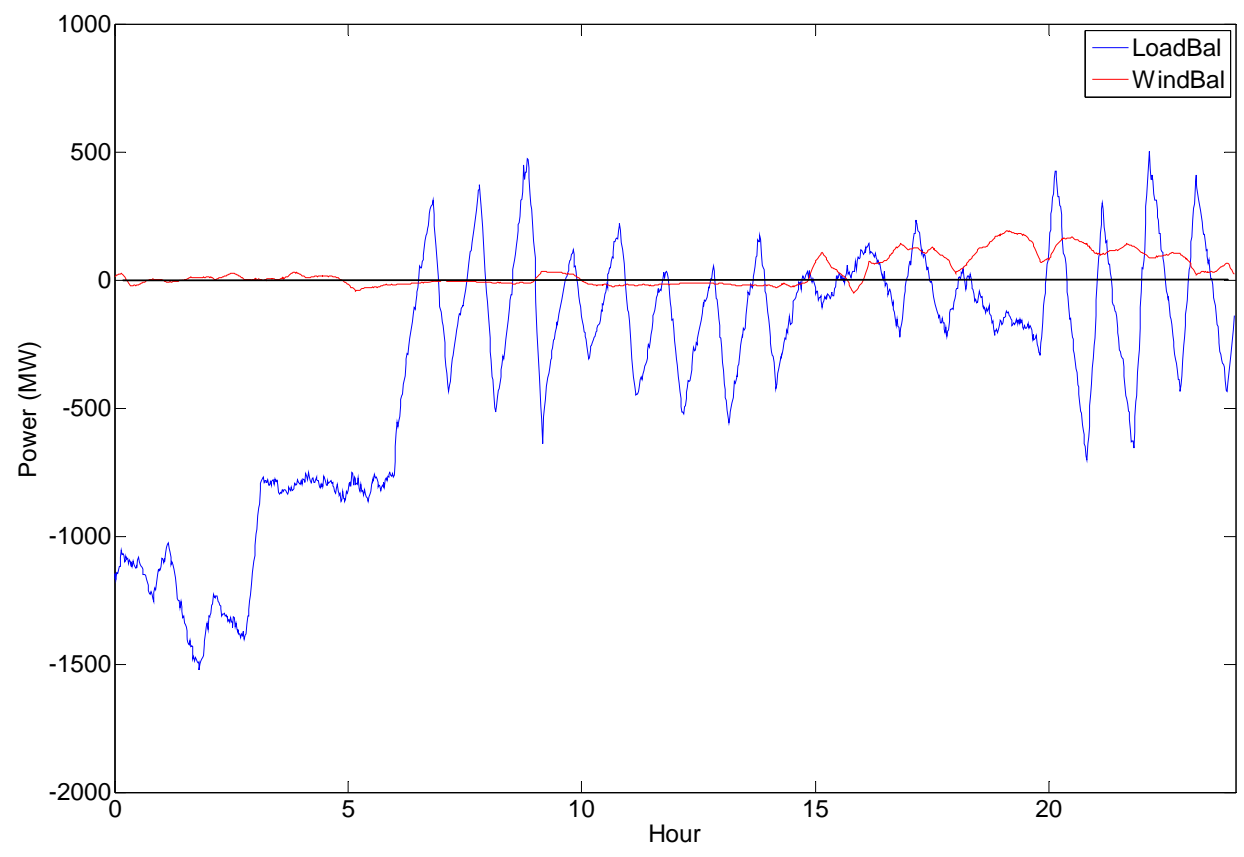

Figure 8.4. One Typical Day Balancing Requirements Caused by Load and Wind Respectively for AZNM

\subsubsection{Energy and Power Requirements}

Extensive systems modeling was performed to estimate the power and energy capacity requirements to meet the future balancing needs. Each technology and technology group required careful simulation that incorporated the specific technical features of a technology, as well as the interaction with each other if more than one technology was modeled. The results of the simulations were a pairing of power (GW) capacity, and energy (GWh) capacity requirements to meet future balancing needs. A detailed discussion of how the technologies were dispatched individually or within an ensemble of other technologies can be found in Appendix B.

Table 8.8 and Figures 8.5 and 8.6 show the results of energy and power requirements for the scenarios in the Southwest (AZNM) area. It should be noted that the capacity requirements or the minimal size of the battery is based on $100 \%$ DOD of the battery. This means that the size of the energy storage is fully utilized. The storage will be cycled from fully charged to fully discharged. As will be discussed, there are good economic reasons for upsizing the battery to a DOD of less than $100 \%$ to improve the life of the battery. For instance, a battery with a DOD of 50\% only uses its energy storage capability to $50 \%$. Significant simulation efforts were performed to determine the minimal capacity (power and energy rating) for the various technology options. The key driver that set the size of the technology was specific operational constraints that force the technology to be operated in a certain way, for instance, the limited change modes and the changeover delay of the pumped hydro and compressed air technologies.

The size for the CT is set by the requirements for generation increment, not the sum of increment and decrement. This is based on the notion that the existing CT capacity is operating at the zero balancing 
point already and would be able to provide generation decrements. Then only the increment in capacity would need to accounted for in the capital cost calculations. This is a very conservative assumption resulting in one-half of the capacity requirements as if one would size the CT to meet the entire amplitude of the balancing requirements from max increment to max decrement. This assumption is still justifiable considering that most CT may not solely be installed for providing balancing services but also participate in the energy markets. If one were to compare the CT technology against storage for its full capacity, then the CT technology must be upside by the magnitude of the maximum decrement, which, in most cases, results in about doubling the size of the CT.

Table 8.8. Power and Energy Requirements for Each Scenario for AZNM. Note: The energy capacity (GWh) for the batteries is nominated at a DOD of $100 \%$.

\begin{tabular}{clcc}
\hline Case & \multicolumn{1}{c}{ Technology } & GW & GWh \\
\hline C1 & Combustion turbine & 1.20 & - \\
C2 & Na-S & 1.22 & 0.46 \\
C3 & Li-ion & 1.22 & 0.46 \\
C4 & Flywheel & 1.21 & 0.42 \\
& CAES & 2.31 & 13.40 \\
C5 & Na-S & 0.64 & 0.06 \\
C6 & Flow battery & 1.23 & 0.48 \\
C7 & PH multiple modes & 1.22 & 0.40 \\
& 4-min waiting period, Na-S & 0.53 & 0.08 \\
C8 & PH 2 modes & 2.31 & 13.47 \\
& 4-min waiting period, Na-S & 0.51 & 0.03 \\
C9 & DR & 4.33 & - \\
\hline
\end{tabular}

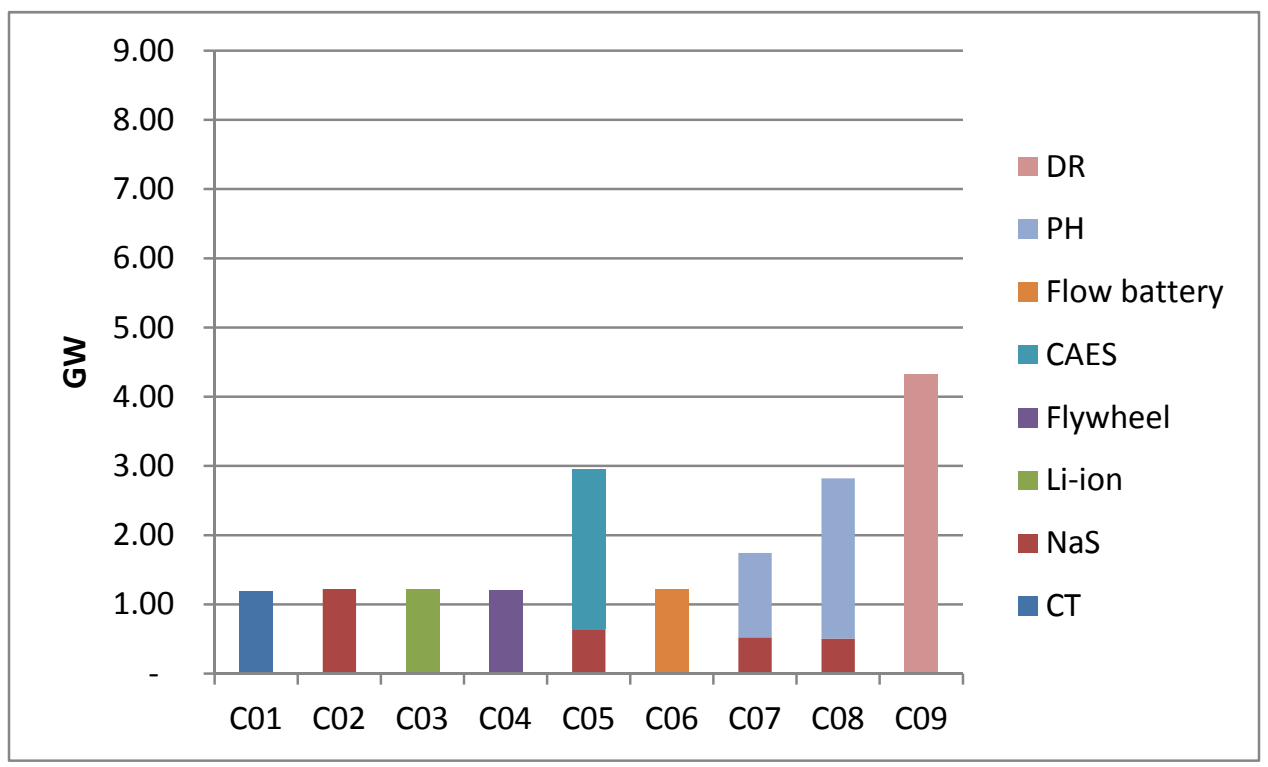

Figure 8.5. Power Requirements for all the Technologies to Meet Balancing Signal for AZNM 


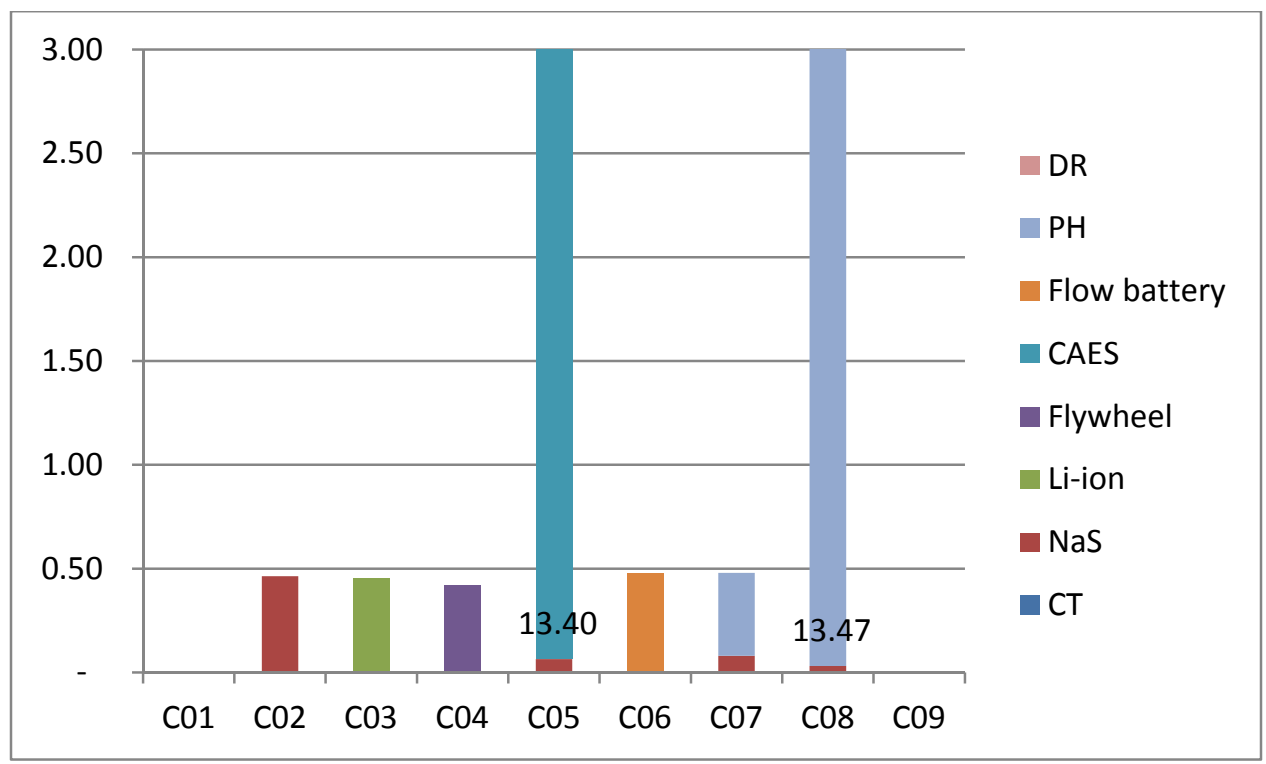

Figure 8.6. Energy Requirements for Storage Technologies to Meet Balancing Signal for AZNM

Notice that there are differences in the sizes of storage (GW and GWh) required for the different cases of studies (see Table 8.8.). These differences are due to the efficiencies and operation strategies of the storage technologies. The GW and GWh difference in cases C2 to C4 and C6 are only due to difference in storage efficiency. The GW and GWh difference in case C7 is due to storage efficiency and due to the need of an additional storage technology (Na-S) to provide balancing during the 4-minute waiting period needed to change between charging and discharging mode (pumping and generation). The large GW and GWh difference in case C5 and C8 with respect to the rest of the cases is mainly because of the restriction in operation assumed; a restriction of only two mode changes (charging to discharging or discharging to charging) is assumed causing a large increase in size requirement (GW and GWh). Details of operation strategies for each technology can be found in Appendix B.

Table 8.9 and Figures 8.6 and 8.7 show the results of energy and power requirements for the scenarios in the Southwest (AZNM) area, considering only the additional wind generation and load expected between 2011 and 2012. These are the requirements for only additional balancing assuming that the 2011 level of balancing is still provided by existing resources. 
Table 8.9. Power and Energy Requirements for Each Scenario due to 2011-2020 Additional Wind and Load Signal for AZNM. Note: The energy capacity (GWh) for the batteries is noted at a DOD of $100 \%$.

\begin{tabular}{clcl}
\hline Case & \multicolumn{1}{c}{ Technology } & GW & GWh \\
\hline C1 & Combustion turbine & 0.21 & - \\
C2 & Na-S & 0.21 & 0.08 \\
C3 & Li-ion & 0.21 & 0.08 \\
C4 & Flywheel & 0.21 & 0.07 \\
C5 & CAES & 0.37 & 1.69 \\
C6 & Flow battery & 0.12 & 0.01 \\
C7 & PH multiple modes & 0.21 & 0.08 \\
& 4 min waiting period, Na-S & 0.21 & 0.07 \\
C8 & PH 2 modes & 0.08 & 0.01 \\
& 4 min waiting period, Na-S & 0.07 & 1.70 \\
C9 & DR & 0.75 & - \\
\hline
\end{tabular}

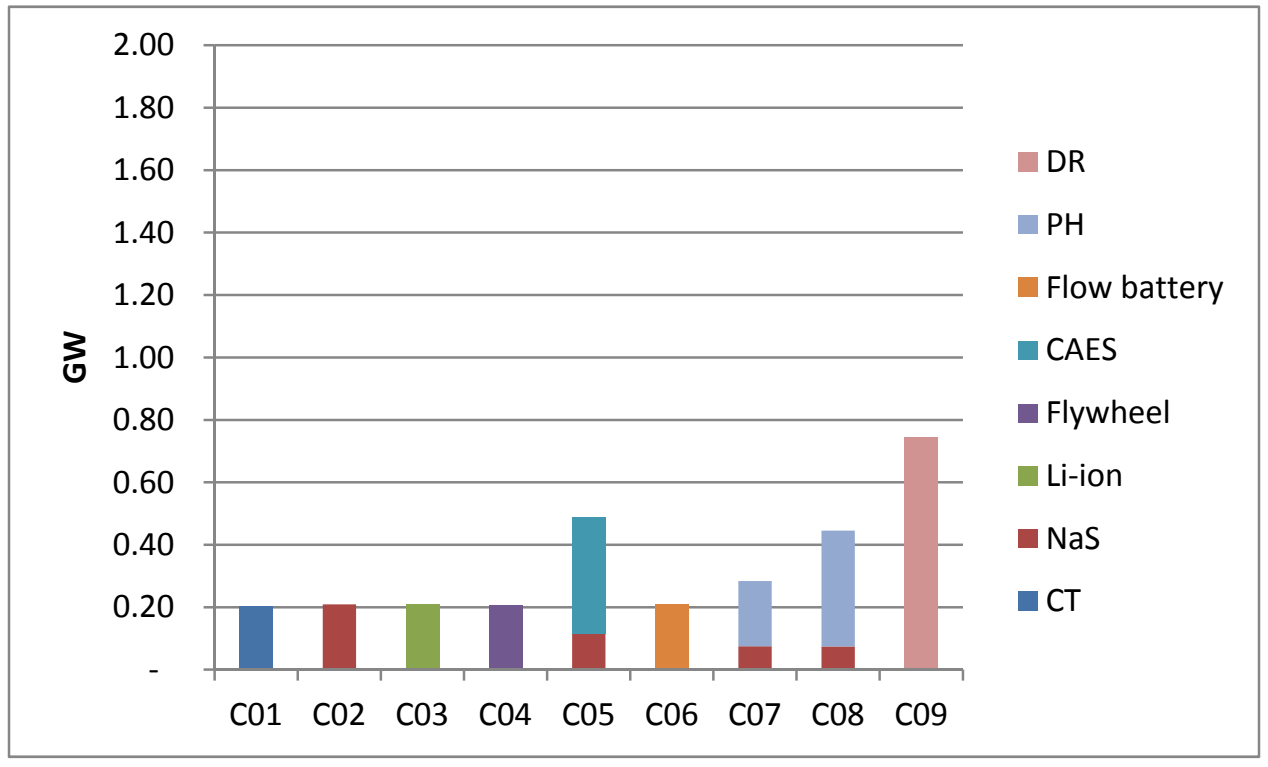

Figure 8.7. Power Requirements for all the Technologies to Meet Balancing Signal due to 2011-2020 Additional Wind and Load Signal for AZNM 


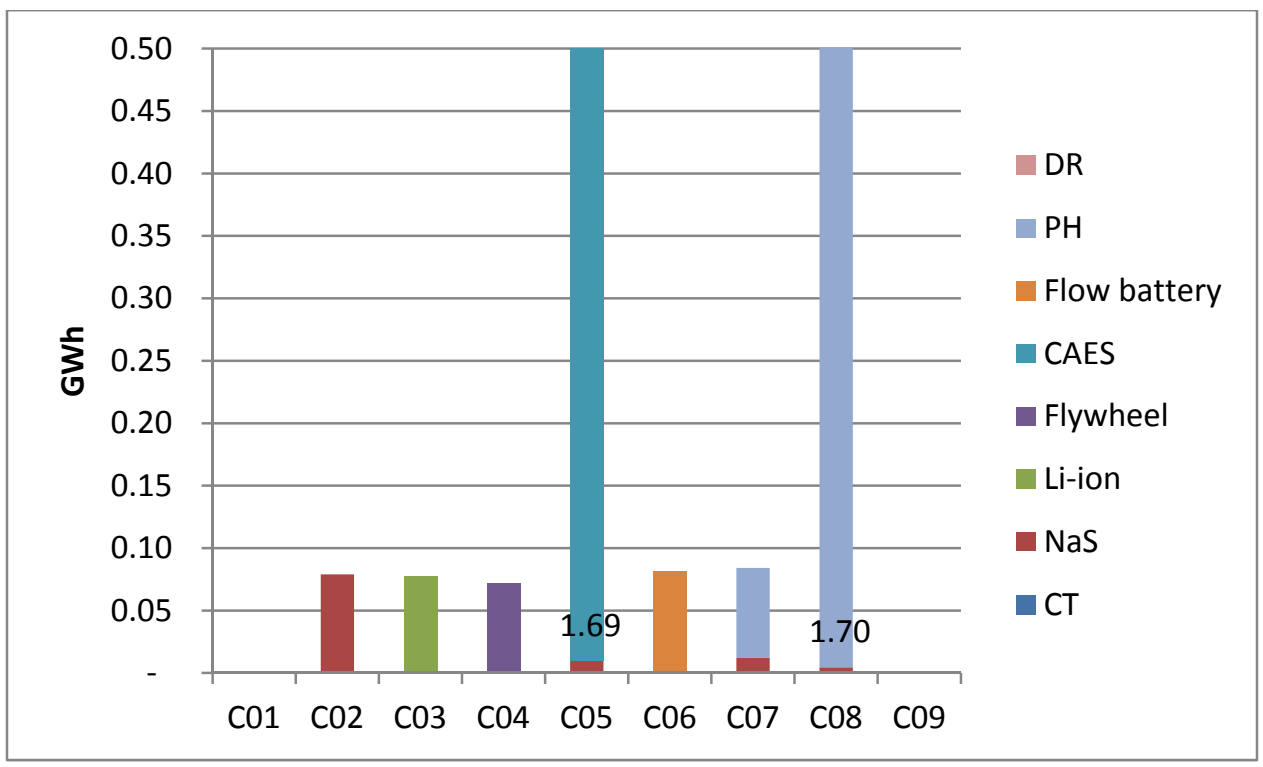

Figure 8.8. Energy Requirements for Storage Technologies to Meet Balancing Signal due to 2011-2020 Additional Wind and Load Signal for AZNM

\subsubsection{Life-Cycle Cost Analysis}

The results of the economic analysis for the AZNM power area are presented in Table 8.10 and Figure 8.9. These results represent the base or reference case for the nine technologies defined in Section 5.1. The values presented in Table 8.10 represent the present value of the stream of capital, O\&M, fuel, and emissions costs over a 50-year time horizon, using the 2020 price values presented in Section 3.3 discounted at $8.0 \%$.

Case 2, which employs Na-S batteries plus CC plants, is the least cost alternative at $\$ 1.9$ billion. Case 4, which consists of flywheels plus CC plants, represents the second least cost alternative with costs estimated at $\$ 2.0$ billion or $9.6 \%$ higher than those estimated for Case 2. The costs associated with the DR-only case (Case 9) are more than twice as expensive as those estimated for the two aforementioned cases, registering at $\$ 4.2$ billion. The CAES case (Case 5) is also more expensive with estimated costs of $\$ 5.9$ billion. In the predominantly $\mathrm{PH}$ case with two mode changes per day (Case 8), total costs are estimated at $\$ 8.0$ billion. Total costs under Case 6 , redox flow batteries plus CC plants, are estimated at $\$ 3.8$ billion. 
Table 8.10. Economic Analysis Results - AZNM (2020 Prices)

\begin{tabular}{ccrcrc}
\hline Case & Capital & Fuel & O\&M & Emissions & Total \\
\hline 1 & 3,185 & 783 & 290 & 309 & $\mathbf{4 , 5 6 7}$ \\
2 & 1,495 & 119 & 200 & 47 & $\mathbf{1 , 8 6 2}$ \\
3 & 2,431 & 107 & 196 & 42 & $\mathbf{2 , 7 7 8}$ \\
4 & 1,609 & 51 & 361 & 20 & $\mathbf{2 , 0 4 1}$ \\
5 & 3,891 & 999 & 586 & 395 & $\mathbf{5 , 8 7 1}$ \\
6 & 3,437 & 138 & 170 & 55 & $\mathbf{3 , 8 0 0}$ \\
7 & 3,832 & 104 & 181 & 41 & $\mathbf{4 , 1 5 8}$ \\
8 & 7,172 & 266 & 451 & 105 & $\mathbf{7 , 9 9 4}$ \\
9 & 4,154 & - & - & - & $\mathbf{4 , 1 5 4}$ \\
\hline
\end{tabular}

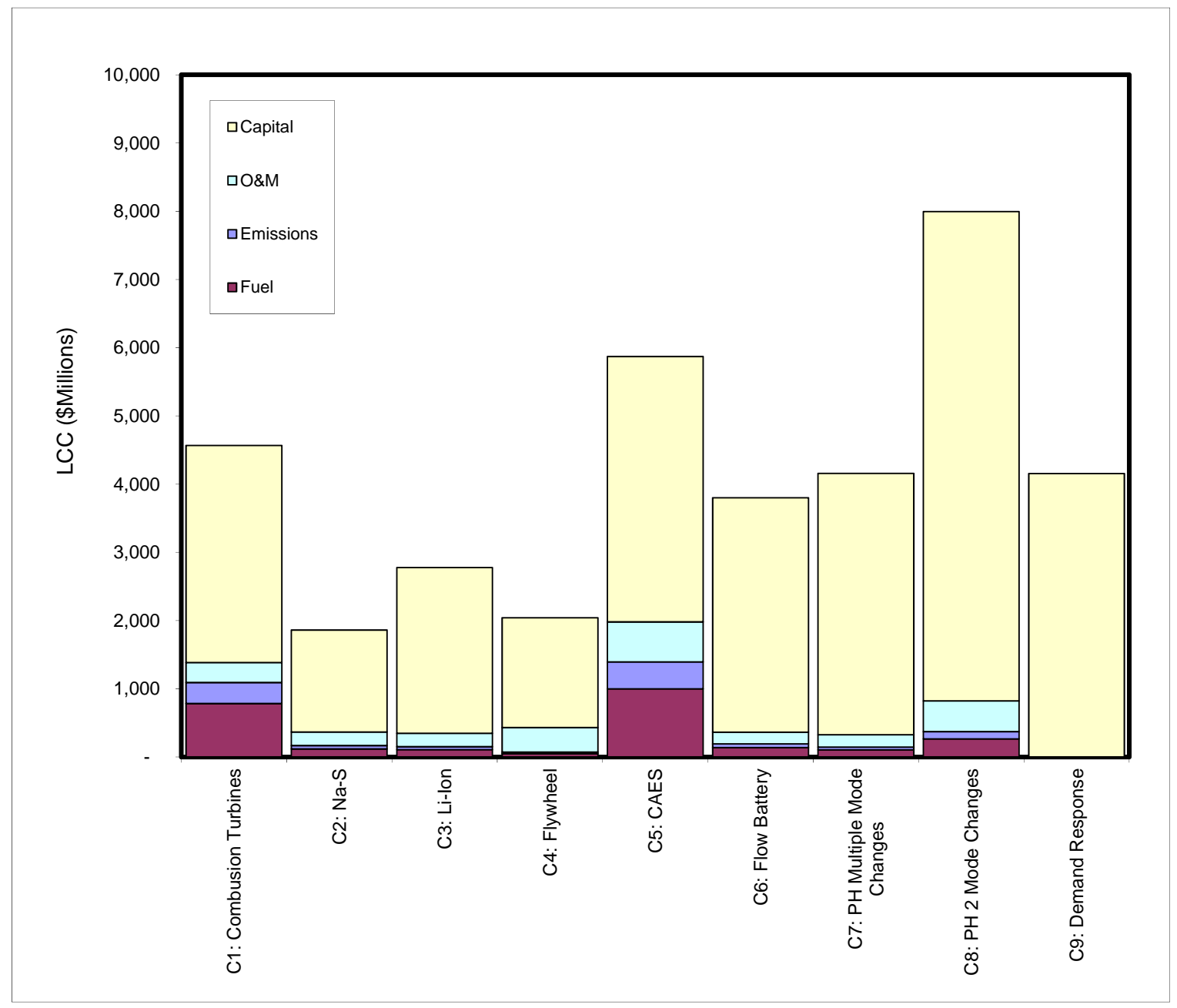

Figure 8.9. Scenario LCC Estimates for AZNM 


\subsubsection{Arbitrage}

Arbitrage analysis was not performed in the AZNM power area.

\subsection{California (CAMX)}

The pattern of balancing signal determines the size of energy storage needed to provide the signal. Specifically, the magnitude of the signal determines the power capacity requirement of energy storage.

\subsubsection{Balancing Requirements}

Figure 8.10 and Figure 8.11 show monthly and daily balancing signals for CAMX, respectively. Long cycles across several days are included in the balancing signal. If only energy storage is used to meet this balancing signal, energy storage that has several days of energy capacity is needed. The long cycle energy storage is very expensive especially for emerging energy storage technologies such as batteries and flywheels. Furthermore, traditional generation resources should have sufficient ramp capability to meet these long cycles because the long cycles usually do not have steep slope. Based on the whole year simulation, the balancing power requirements are $4126 \mathrm{MW}$ of inc. capacity and $2922 \mathrm{MW}$ of dec. capacity, using the BPA's customary $99.5 \%$ probability bound. The balancing requirements for August have a spike which is over the annual inc. capacity, but the spike has a less than $0.5 \%$ probability to happen.

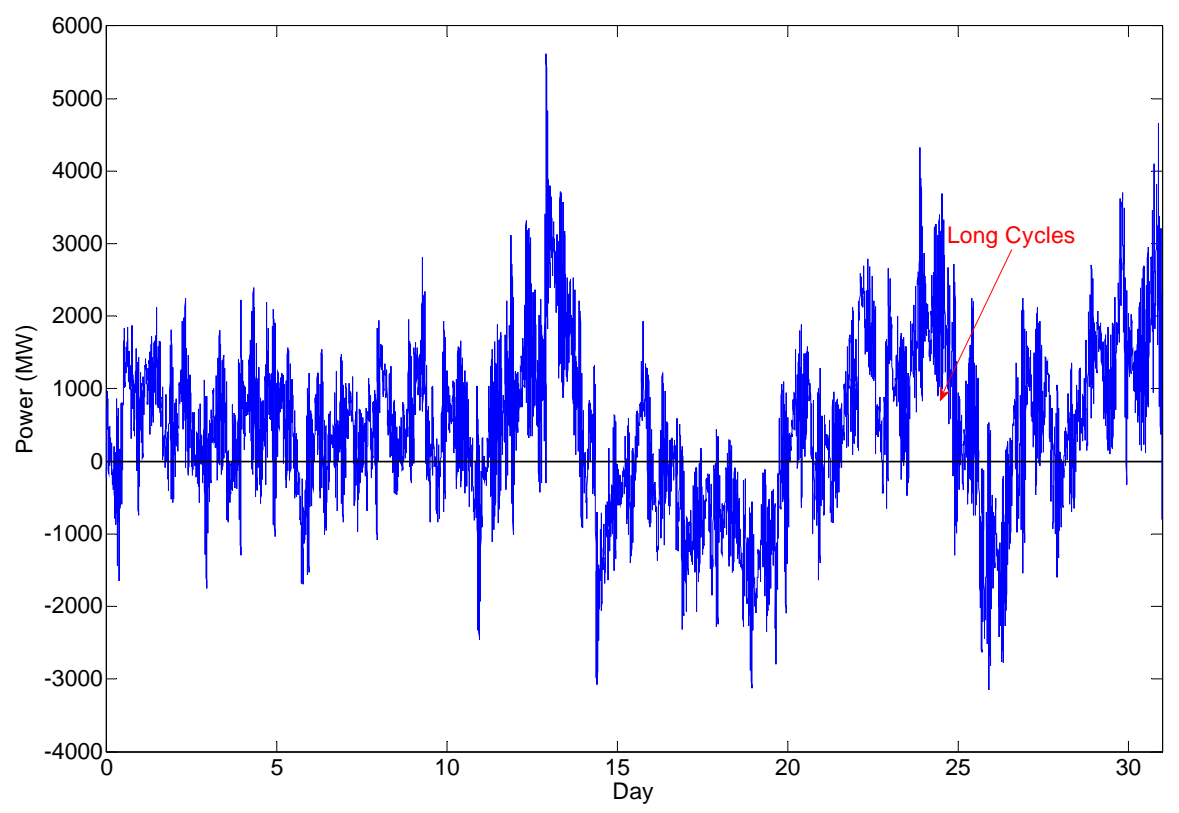

Figure 8.10. One Month Total Balancing Signal of August 2020 for CAMX

Figure 8.10 shows the balancing signal caused by wind and caused by load separately for the whole of August. Figure 8.11 shows the same information for a day in August. For the California region, the balancing requirements are mainly caused by load uncertainty because of the high peak demand in California in 2020. Most of time in August, balancing requirements caused by wind has an opposite sign 
of the balancing requirements caused by load. This indicates if wind production has an over-forecast which means actual wind power production is less than the wind forecast, load will have an underforecast. Given load has positive correlation with temperature and wind has negative correlation with temperature in summer, the results are as we expected.

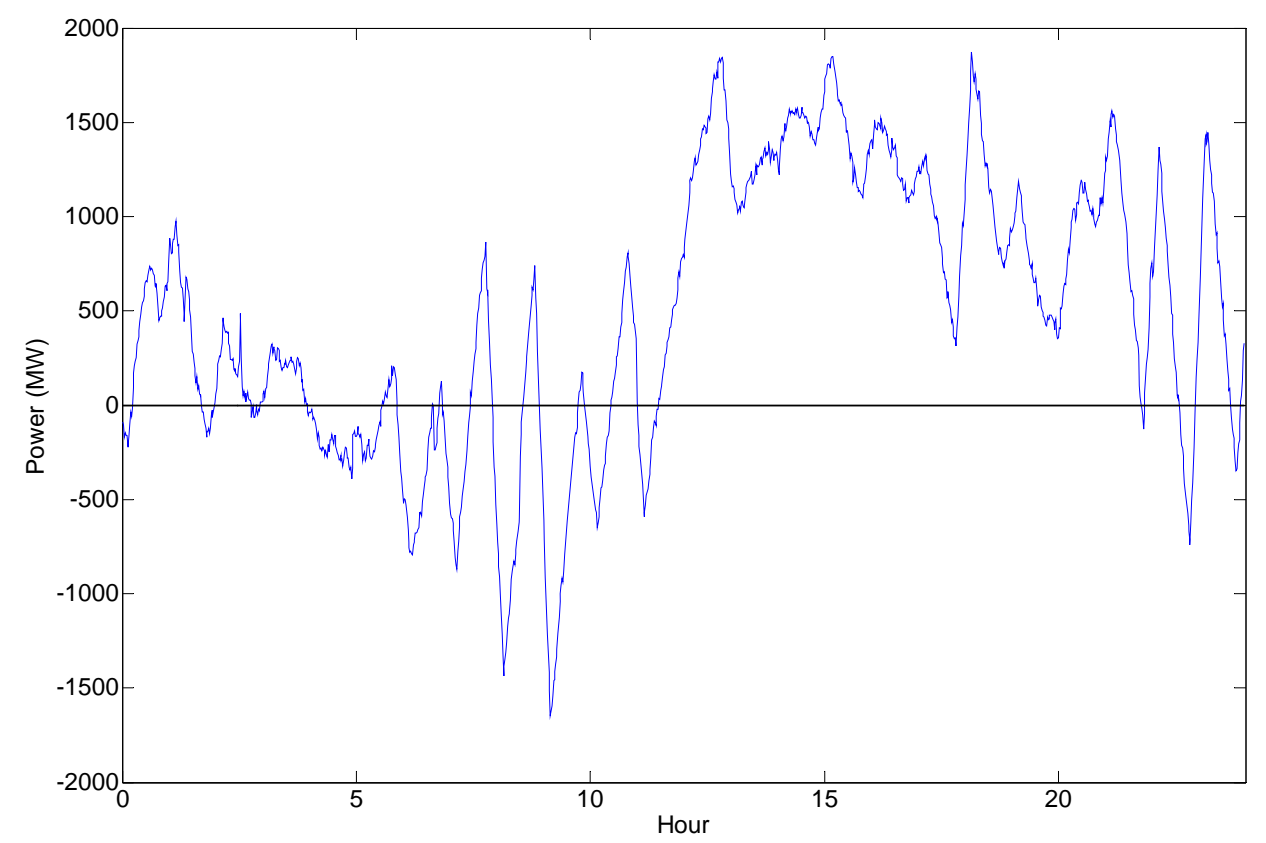

Figure 8.11. One Typical Day Total Balancing Signal of August 2020 for CAMX

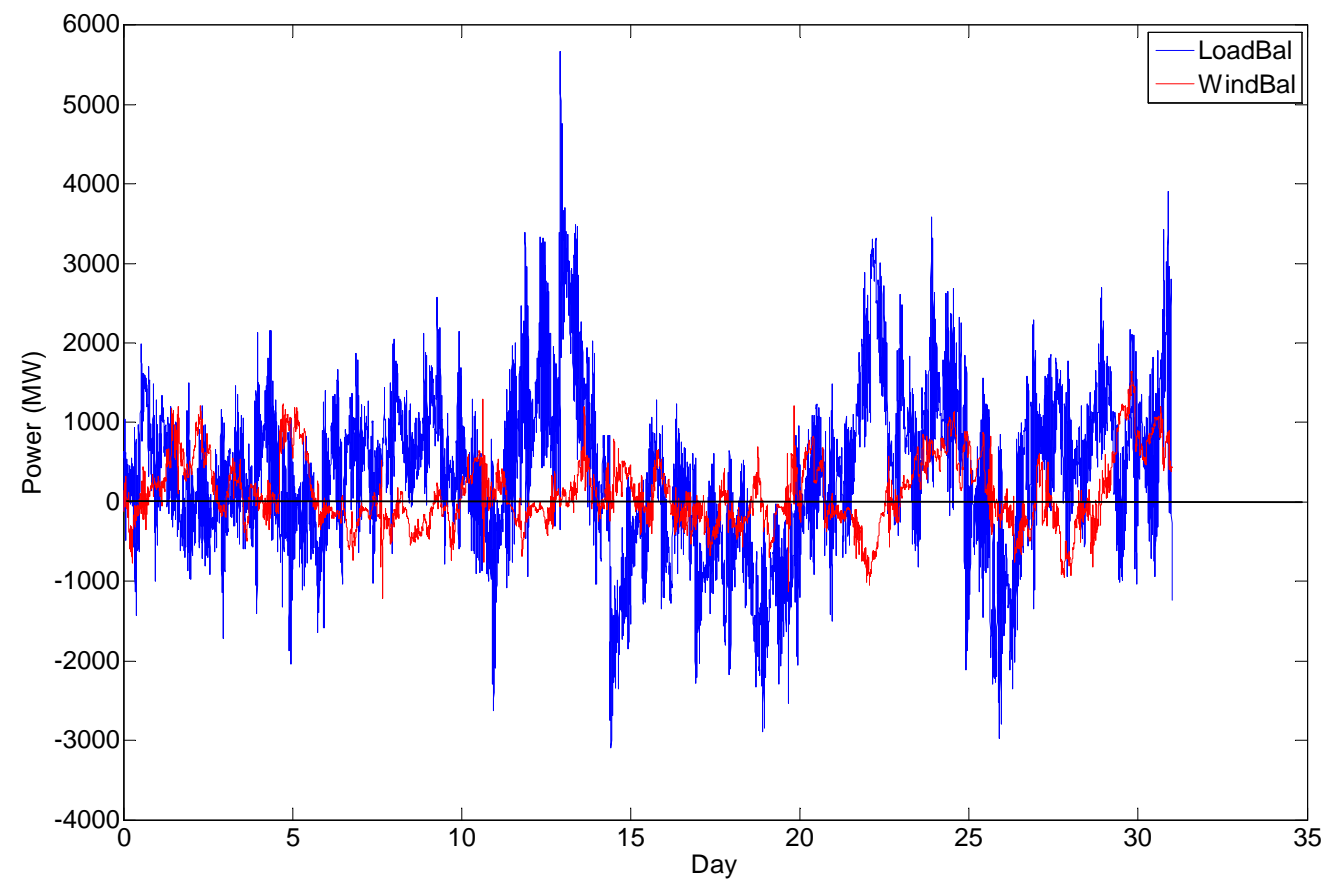

Figure 8.12. One Month Balancing Requirements Caused by Load and Wind, Respectively for CAMX 


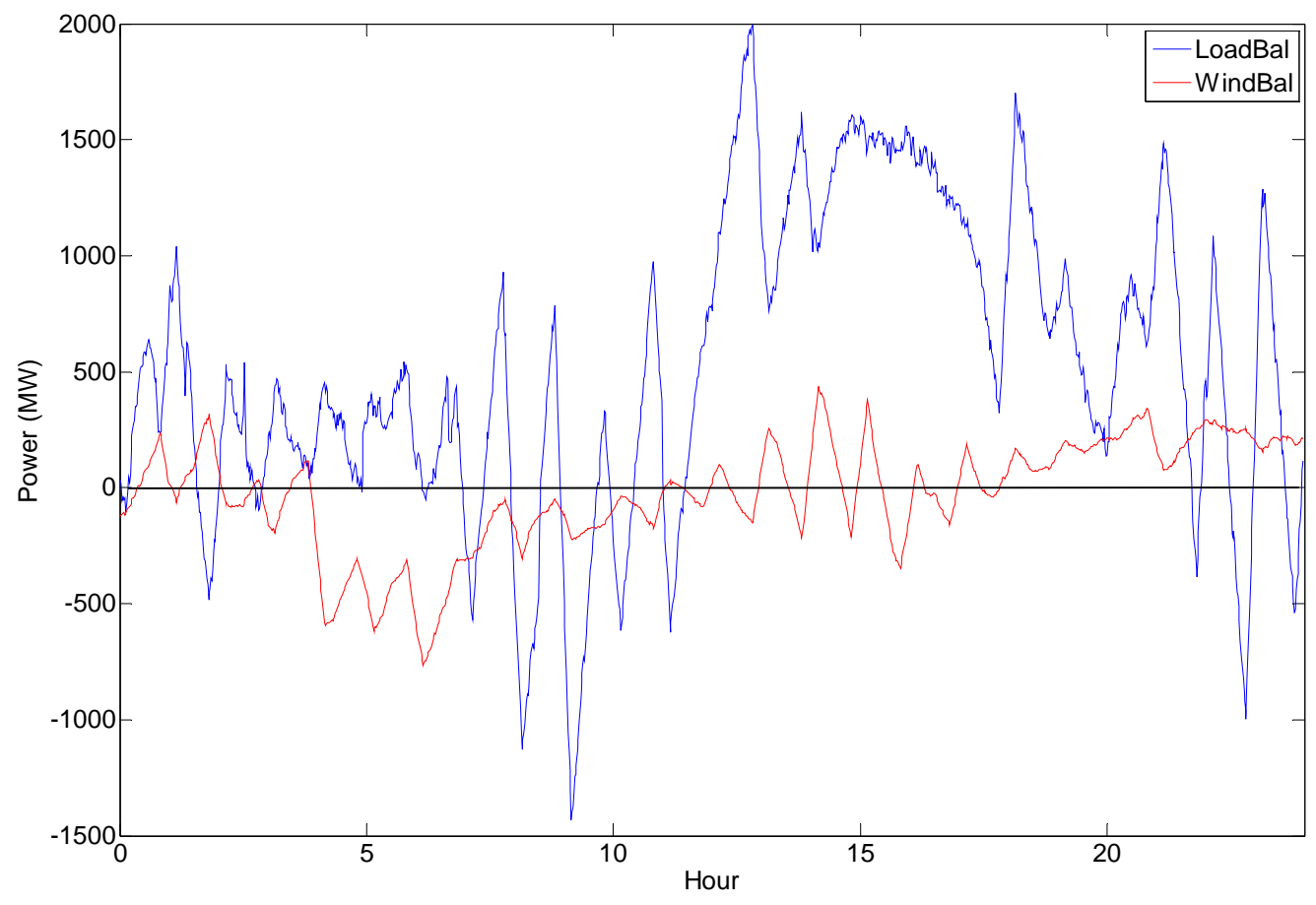

Figure 8.13. One Typical Day Balancing Requirements Caused by Load and Wind, Respectively for CAMX

\subsubsection{Life-Cycle Cost Analysis}

The results of the economic analysis for CAMX are presented in Table 8.11 and Figure 8.14. These results represent the base or reference case for the nine technologies defined in Section 5.1. The values presented in Table 7.6 represent the present value of the stream of capital, O\&M, fuel, and emissions costs over a 50-year time horizon, using the 2020 price values presented in Section 3.3 discounted at $8.0 \%$.

Table 8.11. Economic Analysis Results - California-Mexico Power Area (2020 Prices)

\begin{tabular}{crrrrr}
\hline Case & Capital & Fuel & O\&M & Emissions & Total \\
\hline 1 & 5,971 & 1,391 & 552 & 550 & $\mathbf{8 , 4 6 3}$ \\
2 & 2,827 & 214 & 373 & 85 & $\mathbf{3 , 4 9 9}$ \\
3 & 4,533 & 193 & 366 & 76 & $\mathbf{5 , 1 6 8}$ \\
4 & 3,108 & 91 & 704 & 36 & $\mathbf{3 , 9 3 9}$ \\
5 & 6,912 & 1,781 & 1,042 & 704 & $\mathbf{1 0 , 4 3 9}$ \\
6 & 6,667 & 248 & 330 & 98 & $\mathbf{7 , 3 4 4}$ \\
7 & 7,456 & 187 & 339 & 74 & $\mathbf{8 , 0 5 6}$ \\
8 & 12,838 & 462 & 794 & 183 & $\mathbf{1 4 , 2 7 7}$ \\
9 & 8,120 & - & - & - & $\mathbf{8 , 1 2 0}$ \\
\hline
\end{tabular}


Case 2, which employs Na-S batteries plus CC plants, is the least cost alternative at \$3.5 billion. Case 4, which consists of flywheels plus CC plants, represents the second least cost alternative with costs estimated at $\$ 3.9$ billion or $12.6 \%$ higher than those estimated for Case 2. The costs associated with the DR-only case (Case 9) are more than twice as expensive as those estimated for the two aforementioned cases, registering at $\$ 8.1$ billion. The CAES case (Case 5) is also more expensive with estimated costs of $\$ 10.4$ billion. In the predominantly PH case with two mode changes per day (Case 8), total costs are estimated at $\$ 14.3$ billion. Total costs under Case 6, redox flow batteries plus CC plants, are estimated at $\$ 7.3$ billion.

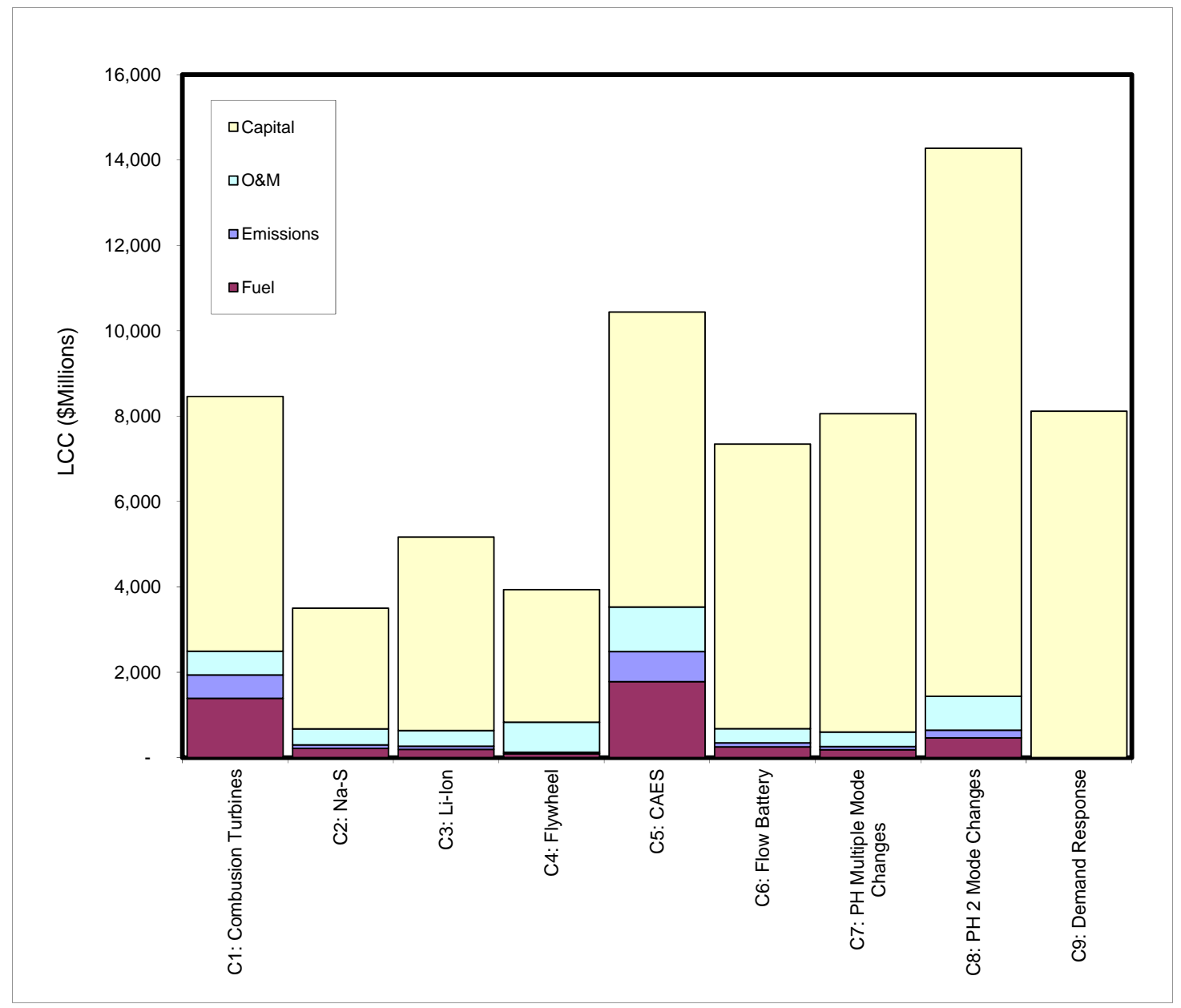

Figure 8.14. Scenario LCC Estimates (California-Mexico Power Area)

\subsubsection{Arbitrage}

Table 8.12 presents the findings of the arbitrage analysis performed for the CAMX power area. As shown, annual revenues are estimated at \$3.9-\$87.3 million based on energy storage size, which ranges from 214-8,542 MW. While the simulation results reveal there are several congested paths in the system, with the most congested path being the interface between Utah and the LADWP, arbitrage revenue expectations fall short of the revenue requirements for each size considered in this analysis. Annualized costs are estimated to range from $\$ 43.3$ million- $\$ 1.7$ billion for pumped hydro, $\$ 97.3$ million- $\$ 3.9$ billion for Na-S, and \$191.3 million-\$7.7 billion for Li-ion. This result supports the conclusion that at a 30\% 
reserve margin, the CAMX power area is not sufficiently congested for energy storage to become costeffective when used to provide only arbitrage services.

Table 8.12. Annualized Capital Costs for Na-S Batteries, Li-Ion Batteries, and Pumped Hydro (CAMX)

\begin{tabular}{|c|c|c|c|c|c|}
\hline \multicolumn{2}{|c|}{ Storage Size } & \multirow{2}{*}{$\begin{array}{l}\text { Annual } \\
\text { Revenue }\end{array}$} & \multicolumn{3}{|c|}{ Annualized Costs } \\
\hline MWh & MW & & Pumped Hydro & $\mathrm{Na}-\mathrm{S}$ & Li-Ion \\
\hline 2,136 & 214 & $\$ 3,877,592$ & $\$ 43,346,379$ & $\$ 97,314,735$ & $\$ 191,276,735$ \\
\hline 4,271 & 427 & $\$ 7,712,931$ & $\$ 86,692,758$ & $\$ 194,629,470$ & $\$ 382,553,470$ \\
\hline 8,542 & 854 & $\$ 14,972,285$ & $\$ 173,385,516$ & $\$ 389,258,940$ & $\$ 765,106,940$ \\
\hline 10,678 & 1,068 & $\$ 18,425,751$ & $\$ 216,731,895$ & $\$ 486,573,675$ & $\$ 956,383,675$ \\
\hline 21,355 & 2,136 & $\$ 33,797,951$ & $\$ 433,463,790$ & $\$ 973,147,350$ & $\$ 1,912,767,350$ \\
\hline 32,033 & 3,203 & $\$ 46,361,474$ & $\$ 650,195,685$ & $\$ 1,459,721,025$ & $\$ 2,869,151,025$ \\
\hline 42,710 & 4,271 & $\$ 58,367,752$ & $\$ 866,927,580$ & $\$ 1,946,294,700$ & $\$ 3,825,534,700$ \\
\hline 53,388 & 5,339 & $\$ 66,402,312$ & $\$ 1,083,659,475$ & $\$ 2,432,868,375$ & $\$ 4,781,918,375$ \\
\hline 85,420 & 8,542 & $\$ 87,323,653$ & $\$ 1,733,855,160$ & $\$ 3,892,589,400$ & $\$ 7,651,069,400$ \\
\hline
\end{tabular}

\subsubsection{Energy and Power Requirements}

Extensive systems modeling was performed to estimate the power and energy capacity requirements to meet the future balancing needs. Each technology and technology group required careful simulation that incorporated the specific technical features of a technology, as well as the interaction with each other if more than one technology was modeled. The results of the simulations were a pairing of power (GW) capacity, and energy (GWh) capacity requirements to meet future balancing needs. A detailed discussion of how the technologies were dispatched individually or within an ensemble of other technologies, can be found in Appendix B.

Table 8.13 and Figures 8.15 and 8.16 show the results of energy and power requirements for the scenarios in the California (CAMX) area, considering only the additional wind generation and load expected between 2011 and 2012. These are the requirements for only additional balancing assuming that the 2011 level of balancing is still provided by existing resources.

For instance, a battery with a DOD of 50\% only uses its nominal energy storage capability to $50 \%$. Significant simulation efforts were performed to determine the minimal capacity (power and energy rating) for the various technology options. The key driver that set the size of the technology was specific operational constraints that force the technology to be operated in a certain way, for instance, the limited change modes and the changeover delay of the pumped hydro and compressed air technologies.

The size for the combustion turbine is set by the requirements for generation increment, not the sum of increment and decrement. This is based on the notion that the existing combustion turbine capacity is operating at the zero balancing point already and would be able to provide generation decrements.

Notice that there are differences in the sizes of storage (GW and GWh) required for the different cases of studies (see Table 8.13.). These differences are due to the efficiencies and operation strategies of the storage technologies. The GW and GWh difference in cases C2 to C4 and C6 are only due to 
difference in storage efficiency. The GW and GWh difference in case C7 is due to storage efficiency and due to the need of an additional storage technology (Na-S) to provide balancing during the 4-minute waiting period needed to change between charging and discharging mode (pumping and generation). The large GW and GWh difference in case C5 and C8 with respect to the rest of the cases is mainly because of the restriction in operation assumed; a restriction of only two mode changes (charging to discharging or discharging to charging) is assumed causing a large increase in size requirement (GW and GWh). (See Table 8.14 and Figures 8.17-18) Details of operation strategies for each technology can be found in Appendix B.

Table 8.13. Power and Energy Requirements for Each Scenario for CAMX. Note: The energy capacity (GWh) for the batteries is nominated at a DOD of $100 \%$.

\begin{tabular}{clcc}
\hline Case & \multicolumn{1}{c}{ Technology } & GW & GWh \\
\hline C1 & Combustion turbine & 2.34 & - \\
C2 & Na-S & 2.40 & 0.67 \\
C3 & Li-ion & 2.39 & 0.66 \\
C4 & Flywheel & 2.36 & 0.64 \\
C5 & CAES & 4.17 & 23.84 \\
& Na-S & 0.90 & 0.10 \\
C6 & Flow battery & 2.41 & 0.68 \\
C7 & PH multiple modes & 2.39 & 0.59 \\
& 4-min waiting period, Na-S & 0.89 & 0.15 \\
C8 & PH 2 modes & 4.17 & 23.90 \\
& 4-min waiting period, Na-S & 0.65 & 0.05 \\
C9 & DR & 8.47 & - \\
\hline
\end{tabular}

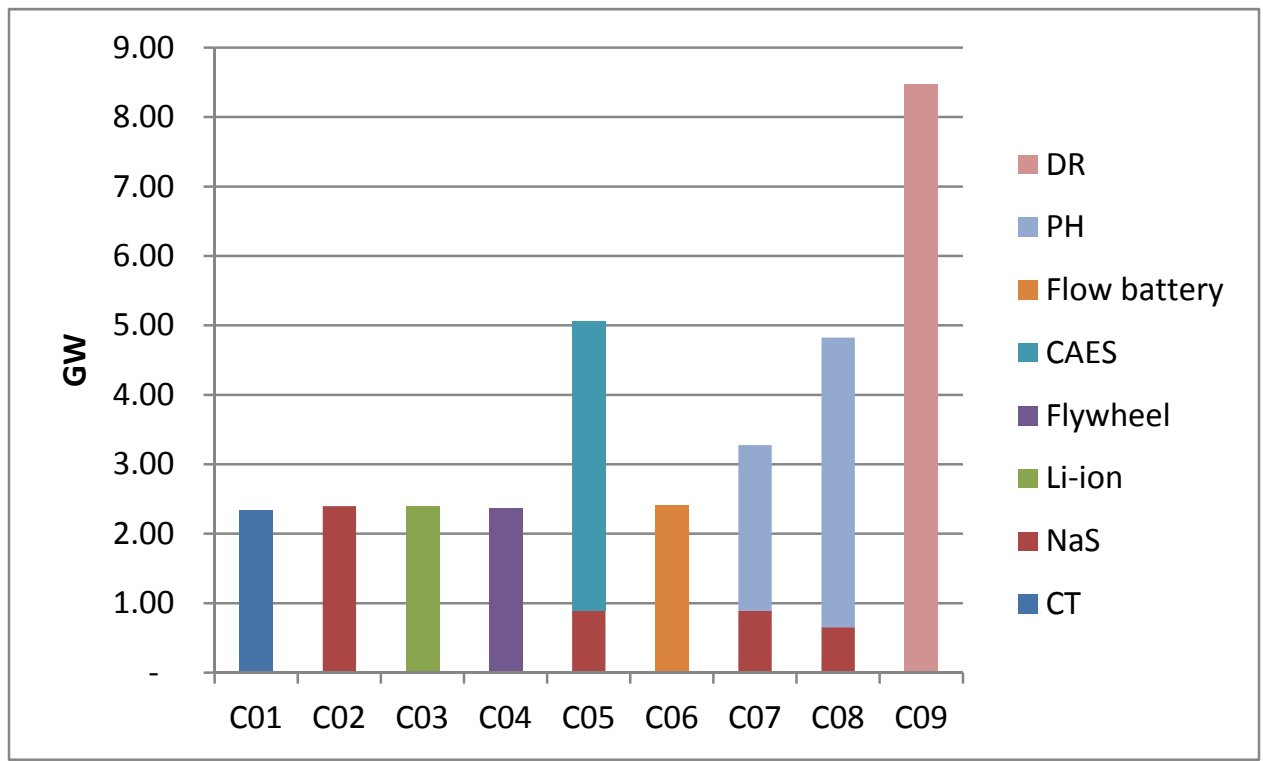

Figure 8.15. Power Requirements for all the Technologies to Meet Balancing Signal for CAMX 


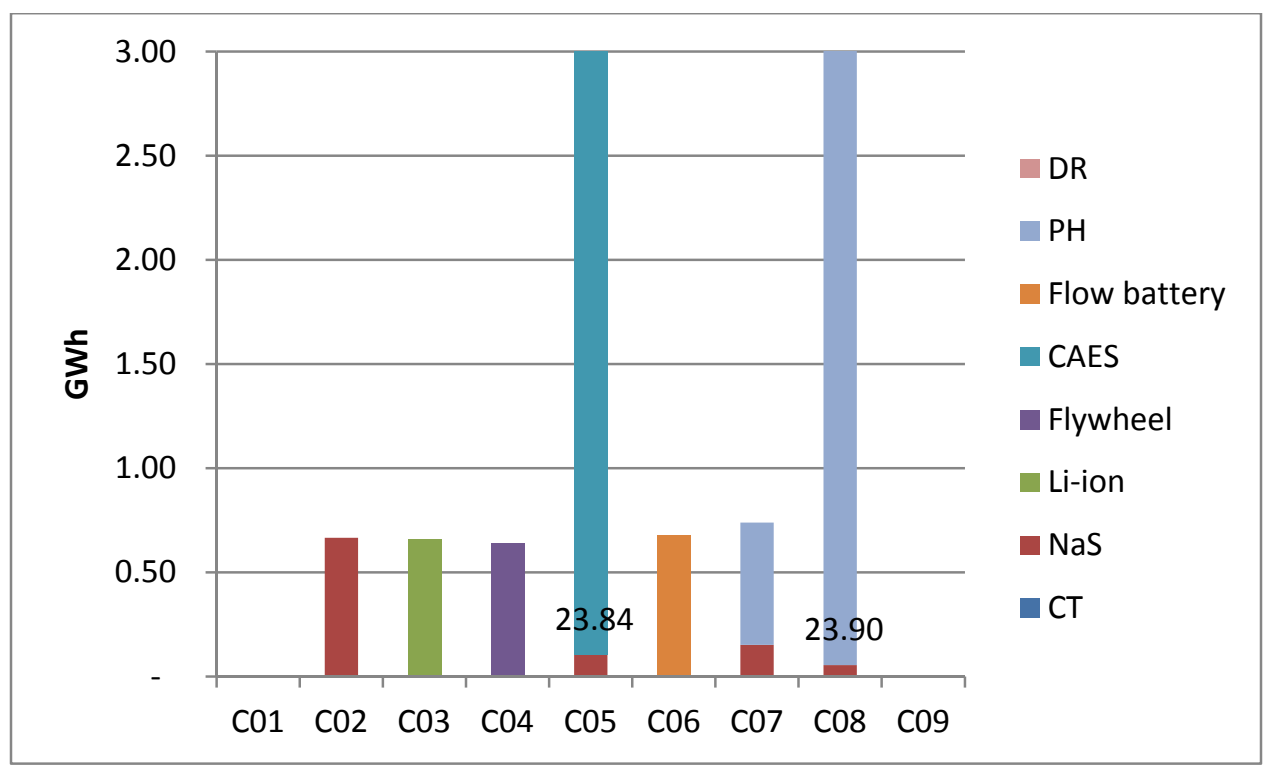

Figure 8.16. Energy Requirements for Storage Technologies to Meet Balancing Signal for CAMX

Table 8.14. Power and Energy Requirements for Each Scenario due to 2011-2020 Additional Wind and Load for CAMX. Note: The energy capacity (GWh) for the batteries is nominated at a DOD of $100 \%$.

\begin{tabular}{clcc}
\hline Case & \multicolumn{1}{c}{ Technology } & GW & GWh \\
\hline C1 & Combustion turbine & 0.53 & - \\
C2 & Na-S & 0.53 & 0.18 \\
C3 & Li-ion & 0.53 & 0.18 \\
C4 & Flywheel & 0.53 & 0.17 \\
C5 & CAES & 0.92 & 6.32 \\
& Na-S & 0.16 & 0.02 \\
C6 & Flow battery & 0.52 & 0.19 \\
C7 & PH multiple modes & 0.53 & 0.17 \\
& 4-min waiting period, Na-S & 0.18 & 0.02 \\
C8 & PH 2 modes & 0.92 & 6.35 \\
& 4-min waiting period, Na-S & 0.10 & 0.01 \\
C9 & DR & 1.53 & - \\
\hline
\end{tabular}




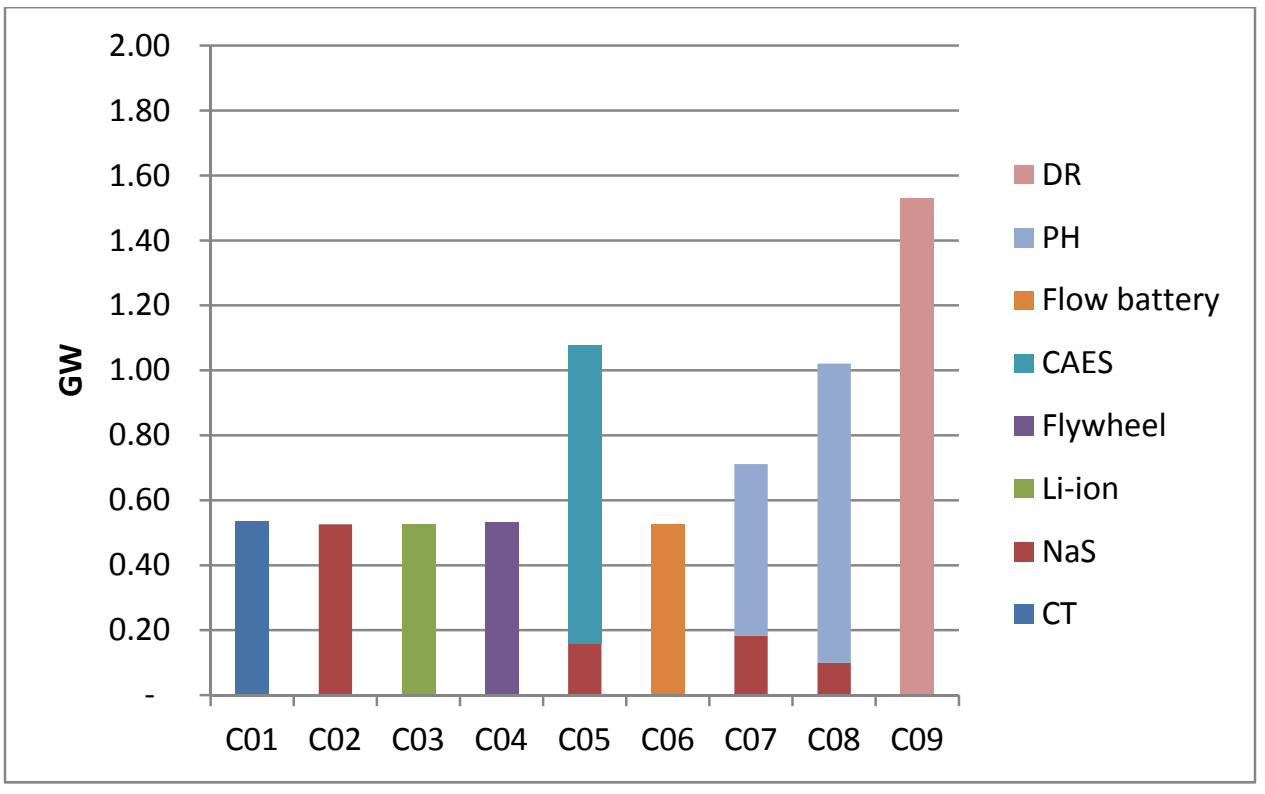

Figure 8.17. Power Requirements for all the Technologies to Meet Balancing Signal due to 2011-2020 Additional Wind and Load for CAMX

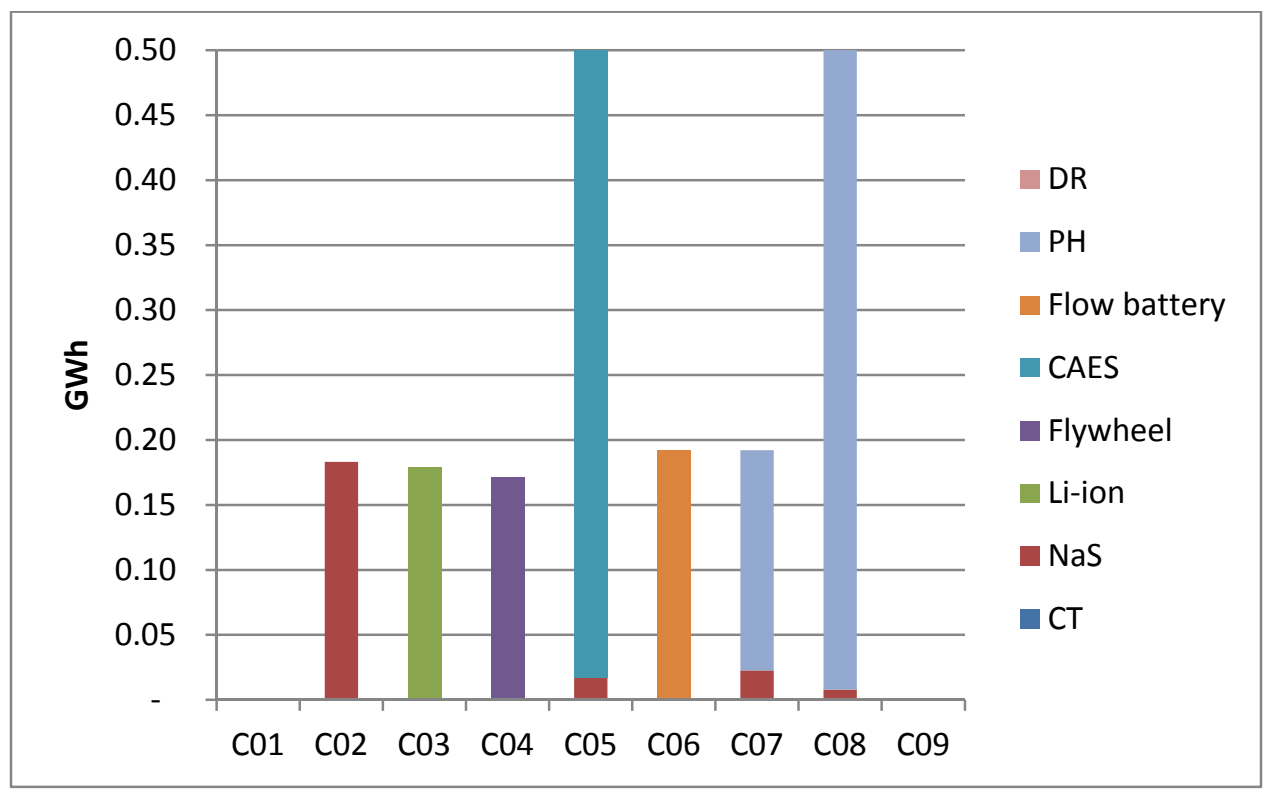

Figure 8.18. Energy Requirements for Storage Technologies to Meet Balancing Signal due to 2011-2020 Additional Wind and Load for CAMX

\subsection{Northwest Power Pool (NWPP)}

\subsubsection{Balancing Requirements}

Figure 8.19 shows balancing signal for NWPP for the whole month of August. Figure 8.20 displays the balancing signal for a day in August. Long cycles across several days are included in the balancing 
signal. If only energy storage is used to meet this balancing signal, energy storage that has several days of energy capacity is needed. The long cycle energy storage is very expensive especially for emerging energy storage technologies such as batteries and flywheels. Furthermore, traditional generation resources should have sufficient ramp capability to meet these long cycles because the long cycles usually do not have steep slope. Based on the whole year simulation, the balancing power requirements are 3431 MW of increased capacity and 2726 MW of decreased capacity, using the BPA's customary 99.5\% probability bound. The balancing requirements for August have a spike which is over the annual increased capacity, but the spike has a probability of less than $0.5 \%$.

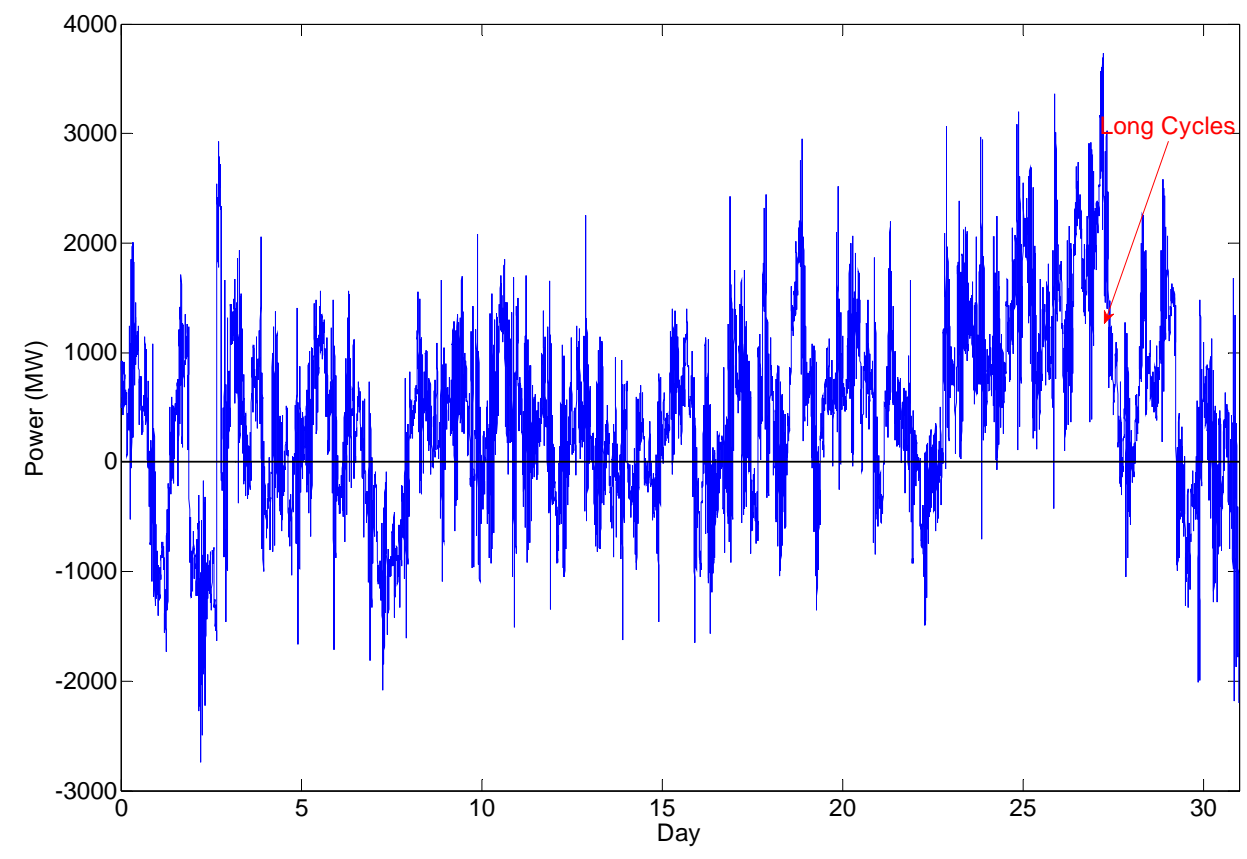

Figure 8.19. One Month Total Balancing Signal of August 2020 for NWPP

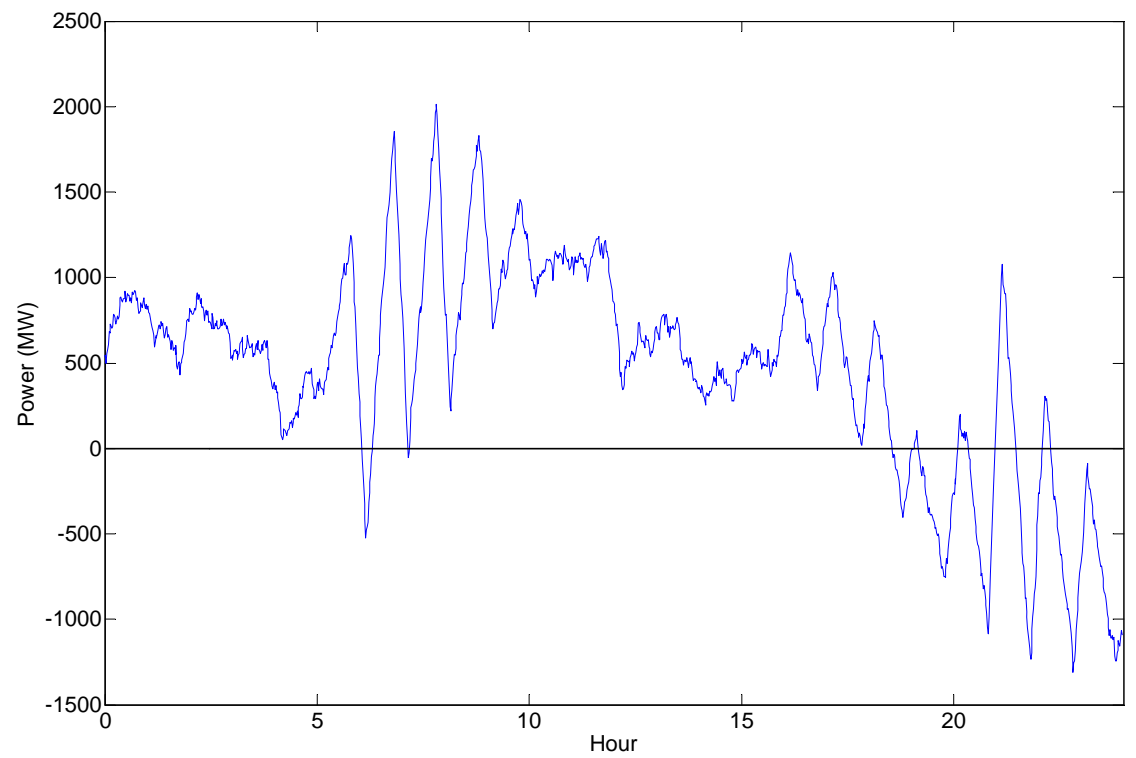

Figure 8.20. One Typical Day Total Balancing Signal of August 2020 for NWPP 
Figure 8.21 shows the balancing signals caused by wind and caused by load for the whole of August. On the third day, a significant load forecast error is observed. But because of the $99.5 \%$ criteria, the balancing signal spike falls into the $0.5 \%$ outliers. Therefore, in the summary table at the beginning of this section, the balancing requirements caused by load are still smaller than the balancing signal caused by wind for NWPP. Figure 8.22 displays the balancing signals for one day in August.

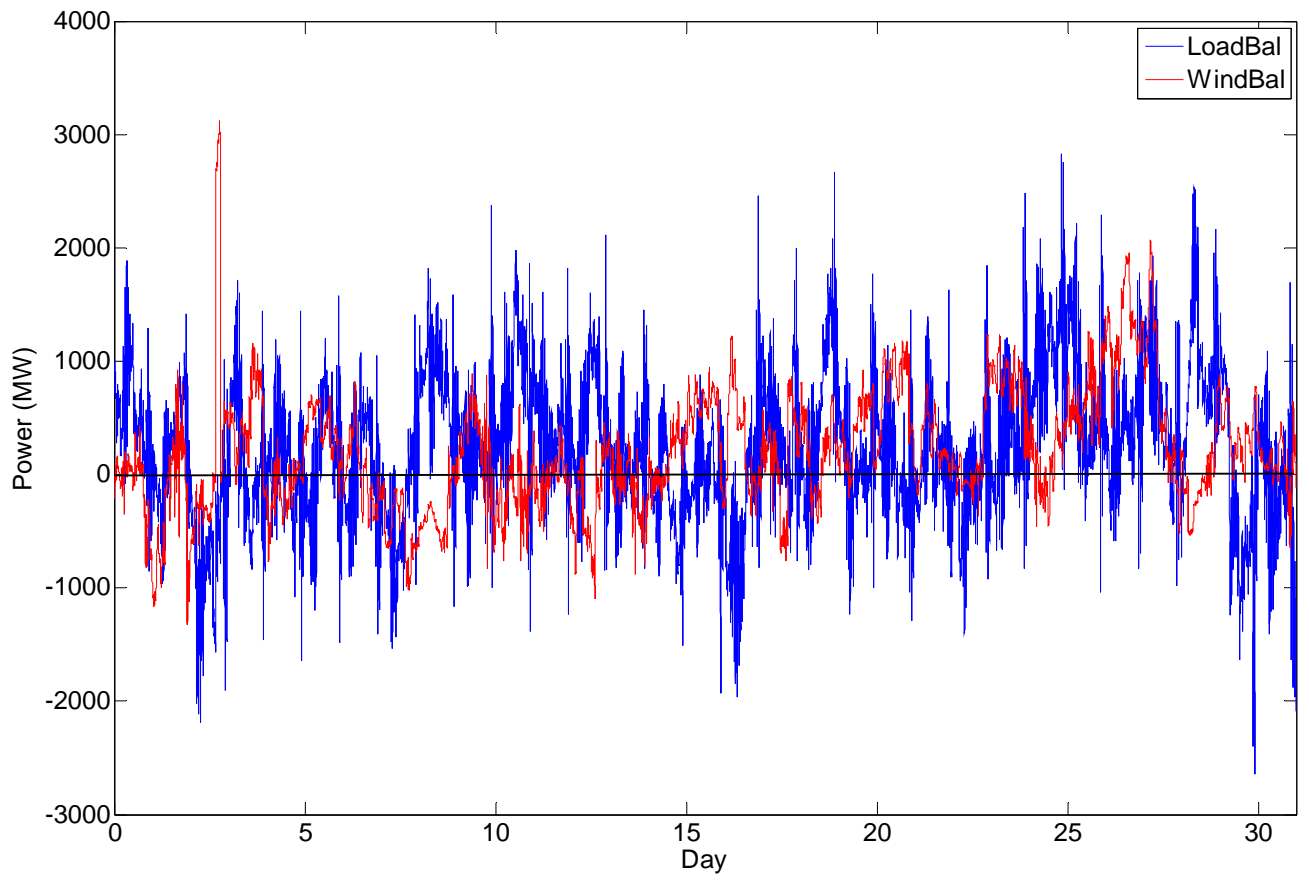

Figure 8.21. One Month Balancing Requirements Caused by Load and Wind, Respectively for NWPP

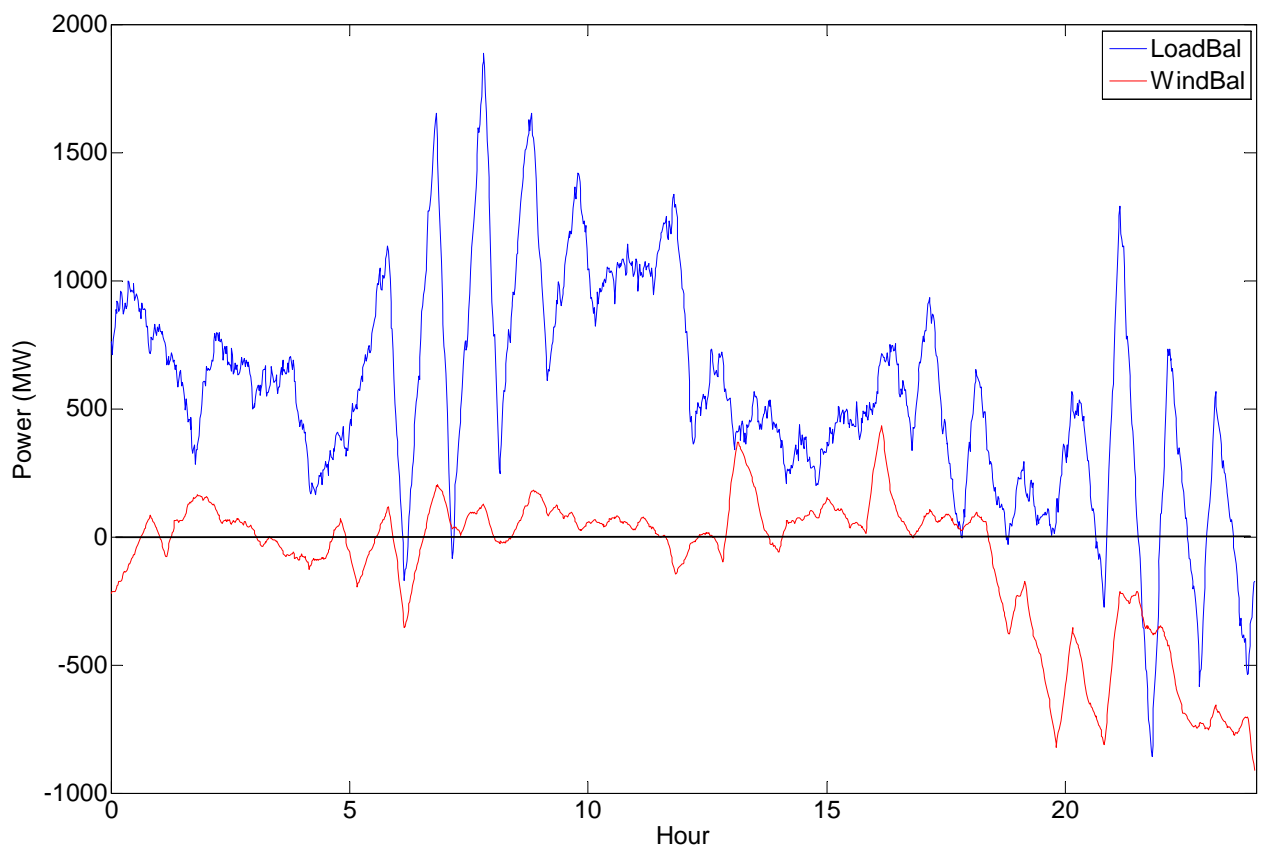

Figure 8.22. One Typical Day Balancing Requirements Caused by Load and Wind, Respectively for NWPP 


\subsubsection{Energy and Power Requirements}

Extensive systems modeling were performed to estimate the power and energy capacity requirements to meet the future balancing needs. Each technology and technology group required careful simulation that incorporated the specific technical features of a technology, as well as the interaction with each other if more than one technology was modeled. The results of the simulations were a pairing of power (GW) capacity, and energy (GWh) capacity requirements to meet future balancing needs. A detailed discussion of how the technologies were dispatched individually or within an ensemble of other technologies can be found in Appendix B.

Table 8.15 and Figures 8.23 and 8.24 show the results of energy and power requirements for the scenarios in the NWPP area. It should be noted that the capacity requirements or the minimal size of the battery is based on $100 \%$ DOD of the battery. This means that the size of the energy storage is fully utilized. The storage will be cycled from fully charged to fully discharged. As will be discussed, there are good economic reasons for upsizing the battery to a DOD of less than $100 \%$ to improve the life of the battery. For instance, a battery with a DOD of 50\% only uses its energy storage capability to $50 \%$. Significant simulation efforts were performed to determine the minimal capacity (power and energy rating) for the various technology options. The key driver that set the size of the technology was specific operational constraints that force the technology to be operated in a certain way, for instance, the limited change modes and the changeover delay of the pumped hydro and compressed air technologies.

The size for the combustion turbine is set by the requirements for generation increment, not the sum of increment and decrement. This is based on the notion that the existing combustion turbine capacity is operating at the zero balancing point already and would be able to provide generation decrements.

Notice that there are differences in the sizes of storage (GW and GWh) required for the different cases of studies (see Table 8.15). These differences are due to the efficiencies and operation strategies of the storage technologies. The GW and GWh differences in cases C2 to C4 and C6 are only due to difference in storage efficiency. The GW and GWh difference in case C7 is due to storage efficiency and due to the need of an additional storage technology (Na-S) to provide balancing during the 4-minute waiting period needed to change between charging and discharging mode (pumping and generation). The large GW and GWh difference in case C5 and C8 with respect to the rest of the cases is mainly because of the restriction in operation assumed; a restriction of only two mode changes (charging to discharging or discharging to charging) is assumed causing a large increase in size requirement (GW and GWh). Details of operation strategies for each technology can be found in Appendix B.

Table 8.16 and Figures 8.25 and 8.26 show the results of energy and power requirements for the scenarios in the Northwest (NWPP) area, considering only the additional wind generation and load expected between 2011 and 2012. These are the requirements for only additional balancing assuming that the 2011 level of balancing is still provided by existing resources. 
Table 8.15. Power and Energy Requirements for Each Scenario for NWPP. Note: The energy capacity (GWh) for the batteries is nominated at a DOD of $100 \%$.

\begin{tabular}{clcc}
\hline Case & \multicolumn{1}{c}{ Technology } & GW & GWh \\
\hline C1 & Combustion turbine & 1.99 & - \\
C2 & Na-S & 2.02 & 0.60 \\
C3 & Li-ion & 2.02 & 0.59 \\
C4 & Flywheel & 2.00 & 0.56 \\
C5 & CAES 2 modes & 3.71 & 22.09 \\
& 7 min waiting period, Na-S & 1.24 & 0.11 \\
C6 & Flow battery & 2.03 & 0.62 \\
C7 & PH multiple modes & 2.01 & 0.58 \\
& 4 min waiting period, Na-S & 0.87 & 0.14 \\
C8 & PH 2 modes & 3.71 & 22.21 \\
& 4 min waiting period, Na-S & 0.89 & 0.05 \\
C9 & DR & 7.19 & - \\
\hline
\end{tabular}

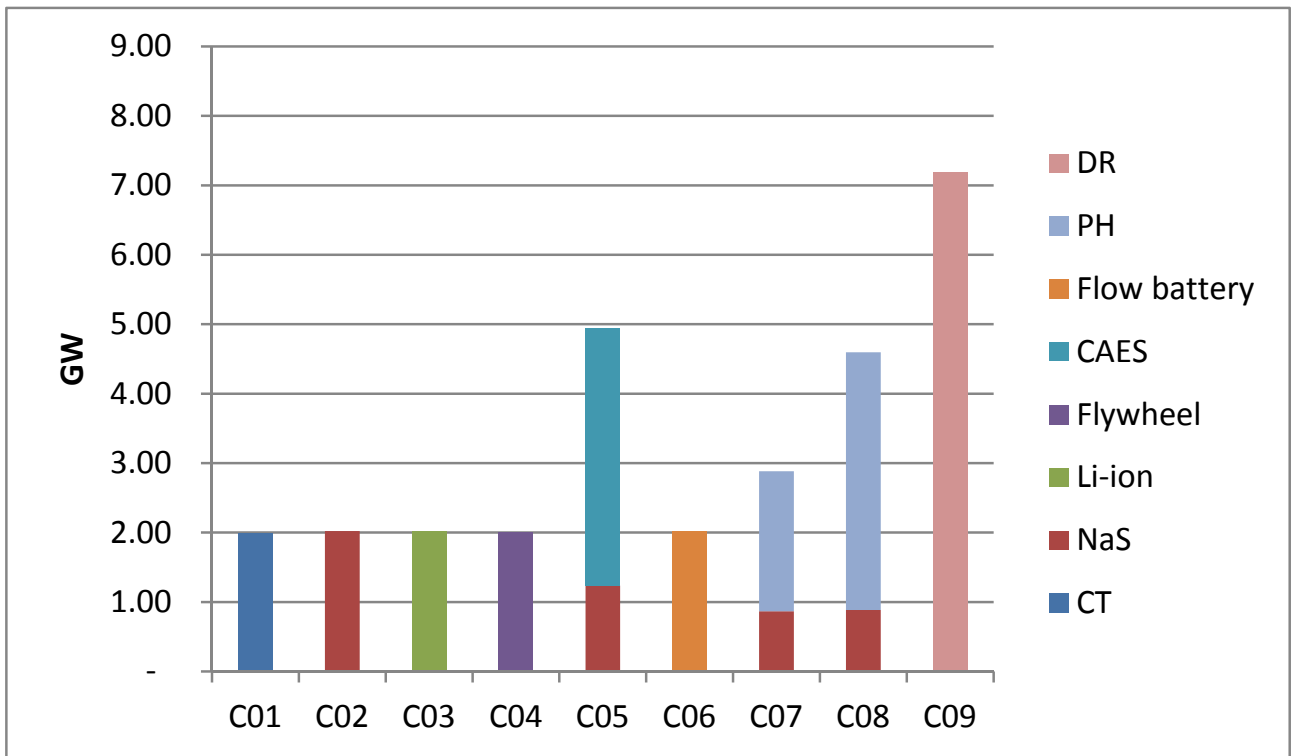

Figure 8.23. Power Requirements for all the Technologies to Meet Balancing Signal for NWPP 


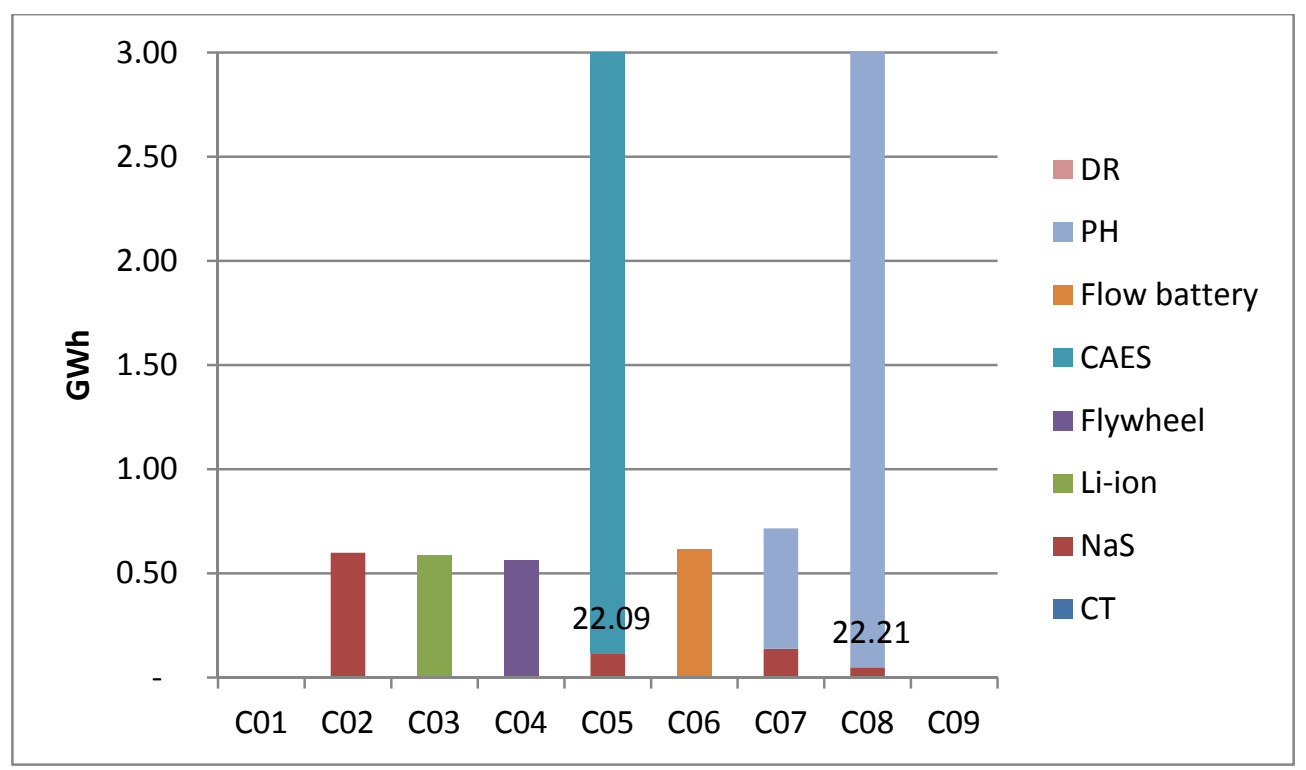

Figure 8.24. Energy Requirements for Storage Technologies to Meet Balancing Signal

Table 8.16. Power and Energy Requirements for Each Scenario due to 2011-2020 Additional Wind and Load for NWPP. Note: The energy capacity (GWh) for the batteries is nominated at a DOD of $100 \%$.

\begin{tabular}{clcc}
\hline Case & \multicolumn{1}{c}{ Technology } & GW & GWh \\
\hline C1 & Combustion turbine & 0.28 & - \\
C2 & Na-S & 0.28 & 0.13 \\
C3 & Li-ion & 0.28 & 0.12 \\
C4 & Flywheel & 0.28 & 0.11 \\
C5 & CAES & 0.52 & 2.84 \\
& Na-S & 0.10 & 0.01 \\
C6 & Flow battery & 0.28 & 0.13 \\
C7 & PH multiple modes & 0.28 & 0.12 \\
& 4-min waiting period, Na-S & 0.10 & 0.02 \\
C8 & PH 2 modes & 0.52 & 2.86 \\
& 4-min waiting period, Na-S & 0.07 & 0.01 \\
C9 & DR & 1.01 & - \\
\hline
\end{tabular}




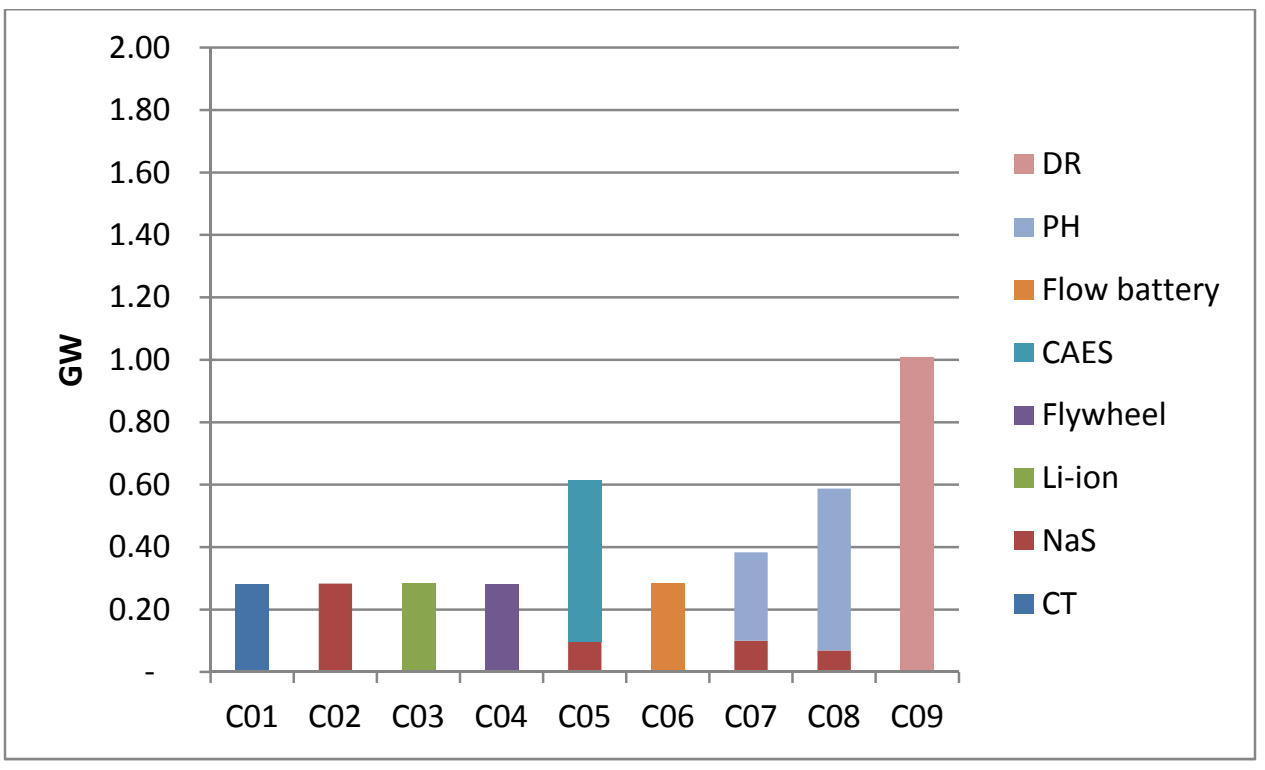

Figure 8.25. Power Requirements for all the Technologies to Meet Balancing Signal due to 2011-2020 Additional Wind and Load for NWPP

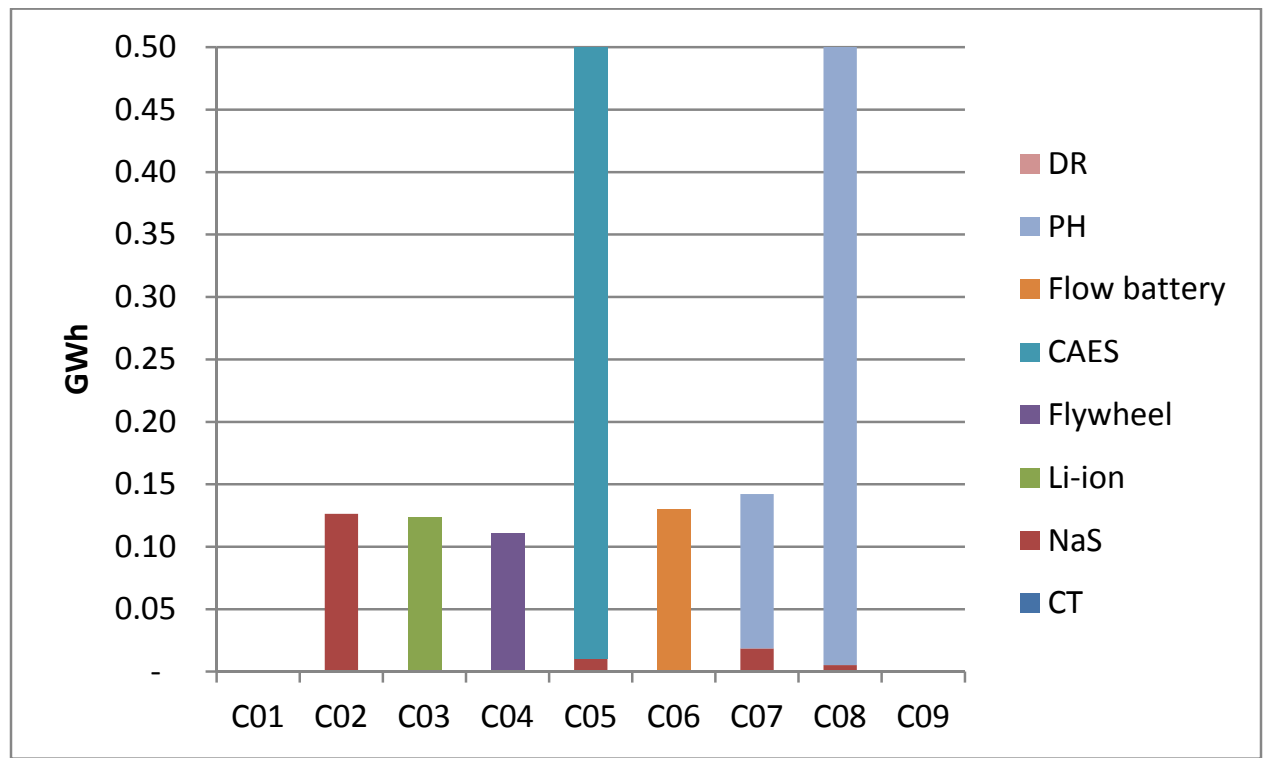

Figure 8.26. Energy Requirements for Storage Technologies to Meet Balancing Signal due to 2011-2020 Additional Wind and Load for NWPP

\subsubsection{Life-Cycle Cost Analysis}

The results of the economic analysis for the NWPP are presented in Table 8.17 and Figure 8.27. These results represent the base or reference case for the nine technologies defined in Section 5.1. The values presented in Table 8.17 represent the present value of the stream of capital, O\&M, fuel, and emissions costs over a 50-year time horizon, using the 2020 price values presented in Section 3.3 discounted at $8.0 \%$. 
Table 8.17. Economic Analysis Results for NWPP (2020 Prices)

\begin{tabular}{crrrrr}
\hline Case & Capital & Fuel & O\&M & Emissions & Total \\
\hline 1 & 5,175 & 1,067 & 454 & 422 & $\mathbf{7 , 1 1 7}$ \\
2 & 2,316 & 164 & 304 & 65 & $\mathbf{2 , 8 4 9}$ \\
3 & 3,884 & 147 & 277 & 58 & $\mathbf{4 , 3 6 6}$ \\
4 & 2,635 & 70 & 592 & 28 & $\mathbf{3 , 3 2 4}$ \\
5 & 6,332 & 1,478 & 903 & 584 & $\mathbf{9 , 2 9 8}$ \\
6 & 5,629 & 190 & 272 & 75 & $\mathbf{6 , 1 6 6}$ \\
7 & 6,334 & 144 & 281 & 57 & $\mathbf{6 , 8 1 7}$ \\
8 & 11,550 & 646 & 776 & 255 & $\mathbf{1 3 , 2 2 7}$ \\
9 & 6,891 & - & - & - & $\mathbf{6 , 8 9 1}$ \\
\hline
\end{tabular}

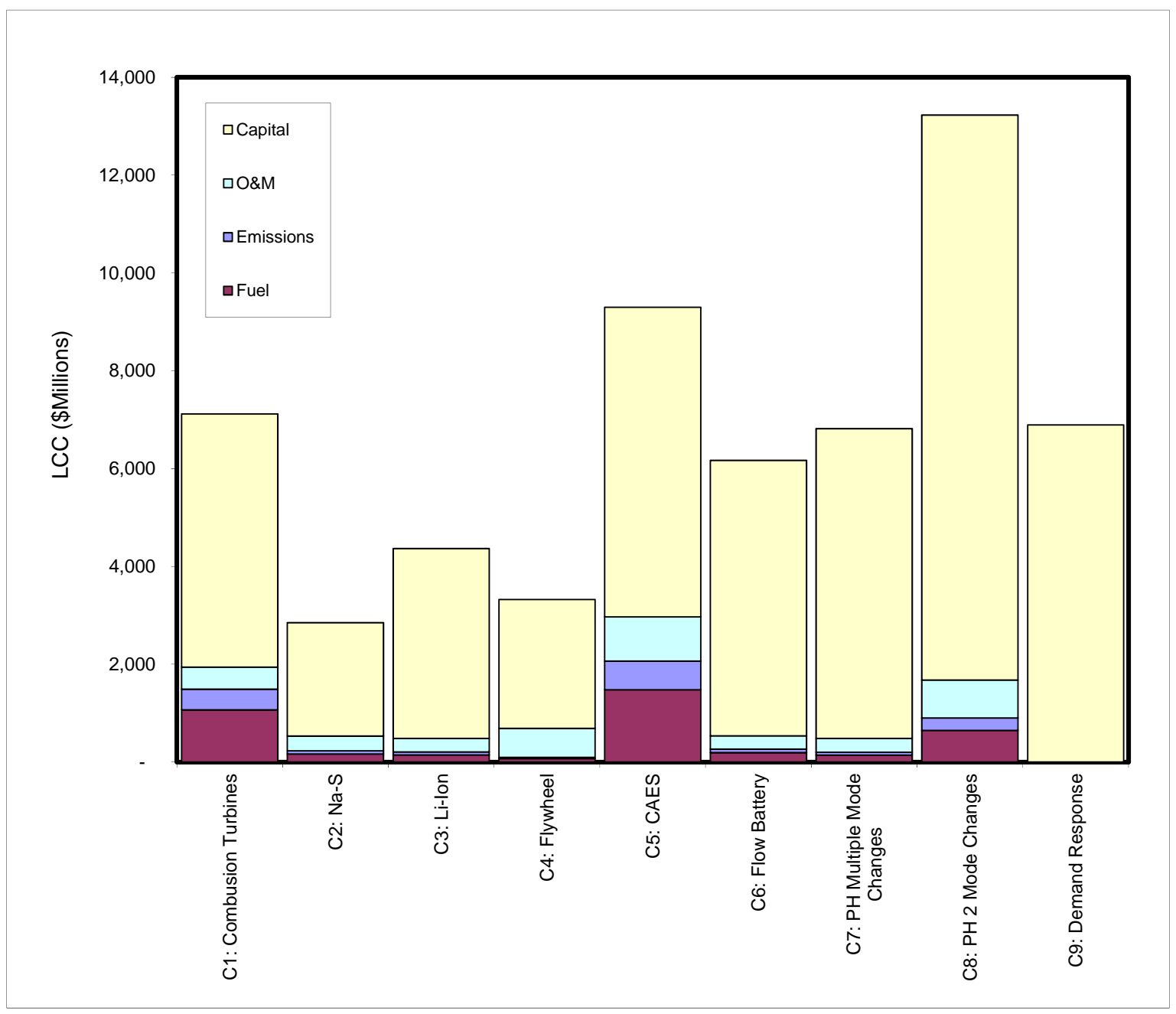

Figure 8.27. Scenario LCC Estimates (NWPP) 
Case 2, which employs Na-S batteries plus CC plants, is the least cost alternative at $\$ 2.8$ billion. Case 4, which consists of flywheels plus CC plants, represents the second least cost alternative with costs estimated at $\$ 3.3$ billion or $16.7 \%$ higher than those estimated for Case 2 . The costs associated with the DR-only case (Case 9) are more than twice as expensive as those estimated for the two aforementioned cases, registering at $\$ 6.9$ billion. The CAES case (Case 5) is also more expensive with estimated costs of $\$ 9.3$ billion. In the predominantly $\mathrm{PH}$ case with two mode changes per day (Case 8), total costs are estimated at $\$ 13.2$ billion. Total costs under Case 6, redox flow batteries plus CC plants, are estimated at $\$ 6.2$ billion.

\subsubsection{Arbitrage}

Table 8.18 presents the findings of the arbitrage analysis performed for the NWPP. As shown, annual revenues are estimated to range from $\$ 0.4-\$ 11.8$ million based on energy storage size, which ranges from 29-1,170 MW. While the simulation results reveal there are congested paths in the system, arbitrage revenue expectations fall short of the revenue requirements for each size considered in this analysis. Annualized costs are estimated to range from \$5.9-\$237.5 million for pumped hydro, \$13.3-\$533.2 million for Na-S, and \$26.2 million-\$1.0 billion for Li-ion. This result supports the conclusion that at a 30\% reserve margin, the NWPP is not sufficiently congested for energy storage to be cost-effective when used to provide only arbitrage services.

Table 8.18. Annualized Capital Costs for Na-S Batteries, Li-Ion Batteries, and Pumped Hydro (NWPP)

\begin{tabular}{|c|c|c|c|c|c|}
\hline \multicolumn{2}{|c|}{ Storage Size } & \multirow{2}{*}{$\begin{array}{c}\text { Annual } \\
\text { Revenue }\end{array}$} & \multicolumn{3}{|c|}{ Annualized Costs } \\
\hline MWh & MW & & Pumped Hydro & $\mathrm{Na}-\mathrm{S}$ & Li-Ion \\
\hline 293 & 29 & $\$ 433,947$ & $\$ 5,937,165$ & $\$ 13,329,225$ & $\$ 26,199,225$ \\
\hline 585 & 59 & $\$ 866,424$ & $\$ 11,874,330$ & $\$ 26,658,450$ & $\$ 52,398,450$ \\
\hline 1,170 & 117 & $\$ 1,710,215$ & $\$ 23,748,660$ & $\$ 53,316,900$ & $\$ 104,796,900$ \\
\hline 1,463 & 146 & $\$ 2,120,051$ & $\$ 29,685,825$ & $\$ 66,646,125$ & $\$ 130,996,125$ \\
\hline 2,925 & 293 & $\$ 4,117,123$ & $\$ 59,371,650$ & $\$ 133,292,250$ & $\$ 261,992,250$ \\
\hline 4,388 & 439 & $\$ 5,968,425$ & $\$ 89,057,475$ & $\$ 199,938,375$ & $\$ 392,988,375$ \\
\hline 5,850 & 585 & $\$ 7,627,850$ & $\$ 118,743,300$ & $\$ 266,584,500$ & $\$ 523,984,500$ \\
\hline 7,313 & 731 & $\$ 8,835,563$ & $\$ 148,429,125$ & $\$ 333,230,625$ & $\$ 654,980,625$ \\
\hline 11,700 & 1,170 & $\$ 11,810,694$ & $\$ 237,486,600$ & $\$ 533,169,000$ & $\$ 1,047,969,000$ \\
\hline
\end{tabular}

\subsection{Rocky Mountain Power Area (RMPA)}

The pattern of balancing signal determines the size of energy storage needed to provide the signal. Specifically, the magnitude of the signal determines the power capacity requirement of energy storage.

\subsubsection{Balancing Requirements}

Figure 8.28 and Figure 8.29 show the balancing signal for the whole August and one day in August, respectively. Long cycles across several days are included in the balancing signal. If only energy storage is used to meet this balancing signal, energy storage that has several days of energy capacity is needed. The long cycle energy storage is very expensive especially for emerging energy storage technologies such 
as batteries and flywheels. Furthermore, traditional generation resources should have sufficient ramp capability to meet these long cycles because the long cycles usually do not have steep slope. Based on the whole year simulation, the balancing power requirements are $2149 \mathrm{MW}$ of increased capacity and 1629 MW of decreased capacity, using the BPA’s customary 99.5\% probability bound. The balancing requirements for August, especially the increased capacity, are lower than the annual requirements.

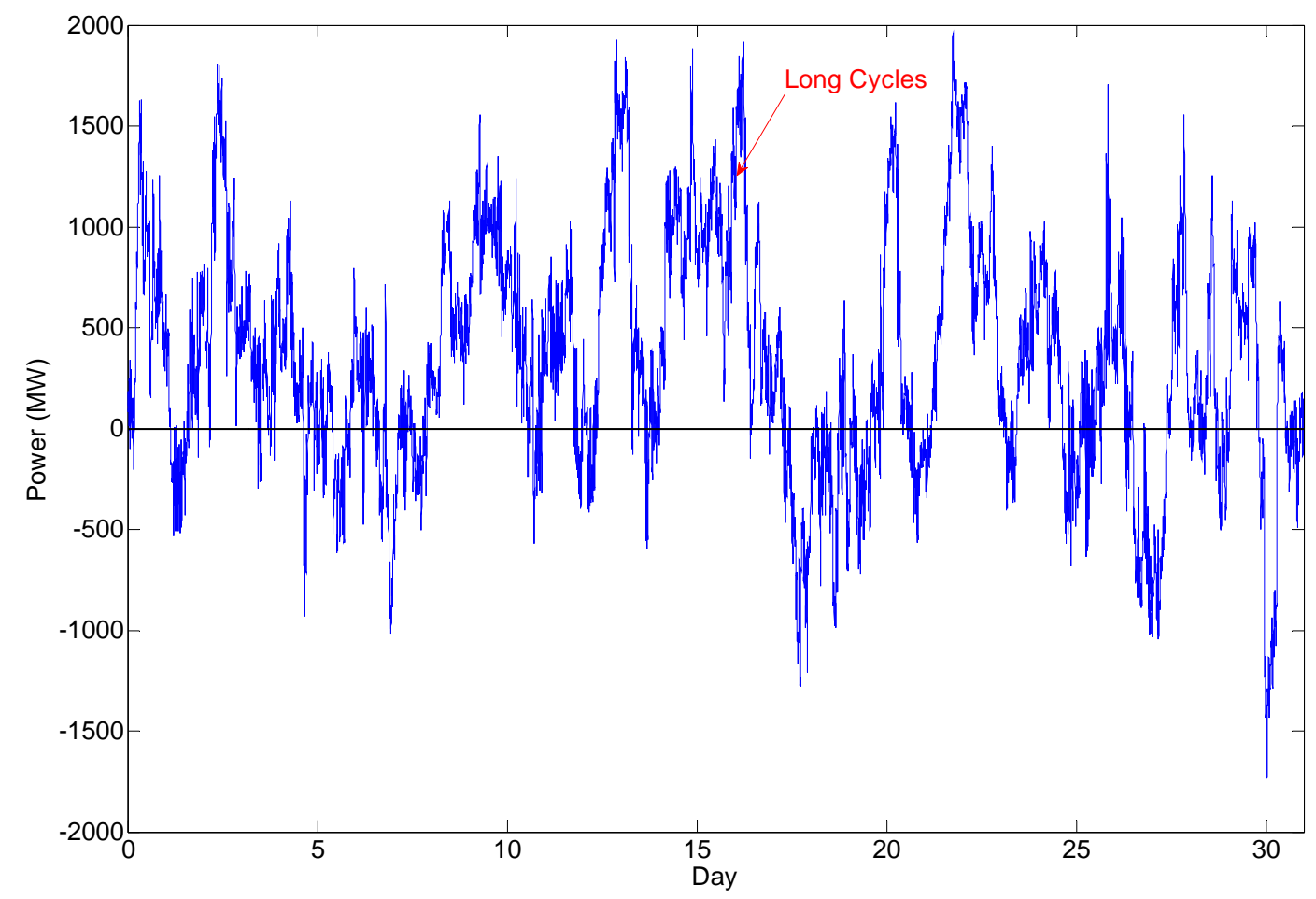

Figure 8.28. One Month Total Balancing Signal of August 2020 for RMPP

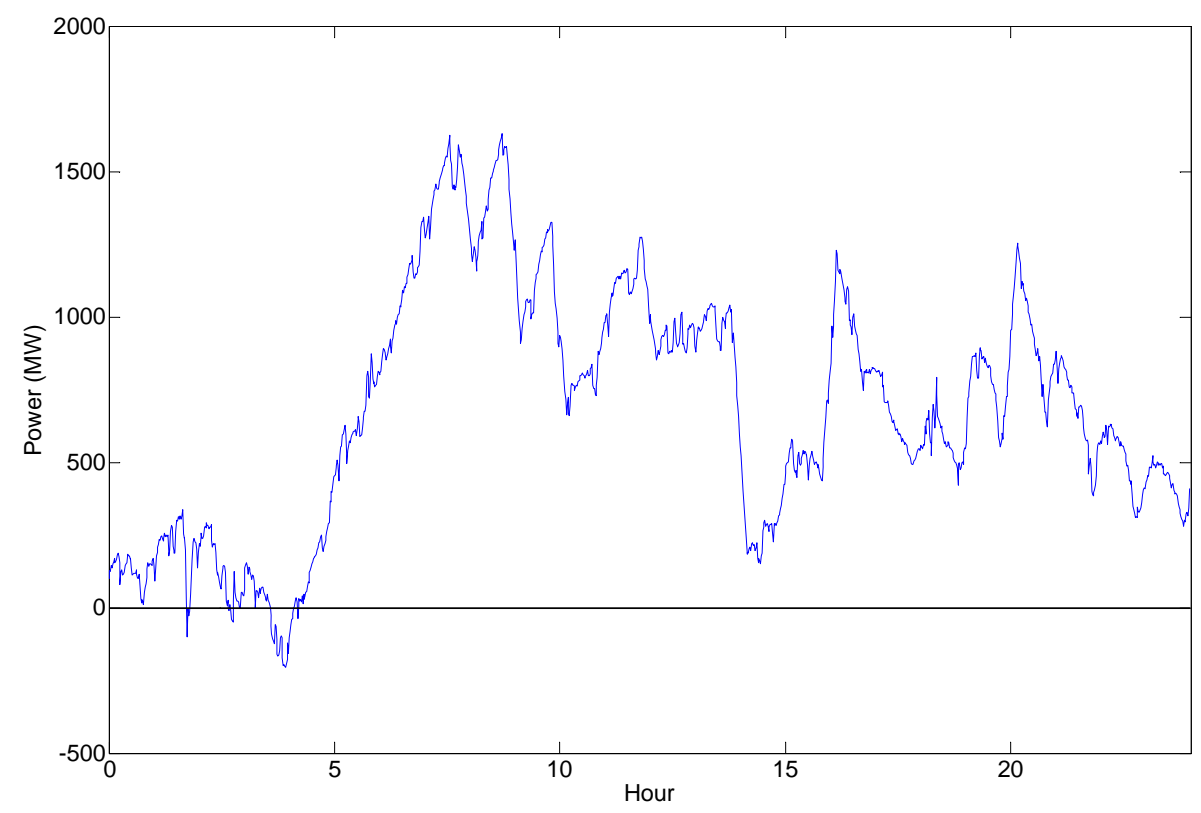

Figure 8.29. One Typical Day Total Balancing Signal of August 2020 for RMPP 
Figure 8.30 shows the balancing signals caused by wind uncertainty and caused by load uncertainty for the whole month of August. The balancing requirements are almost evenly caused by wind uncertainty and load uncertainty for RMPA. Figure 8.31 displays one day balancing signals caused by wind uncertainty and caused by load uncertainty.

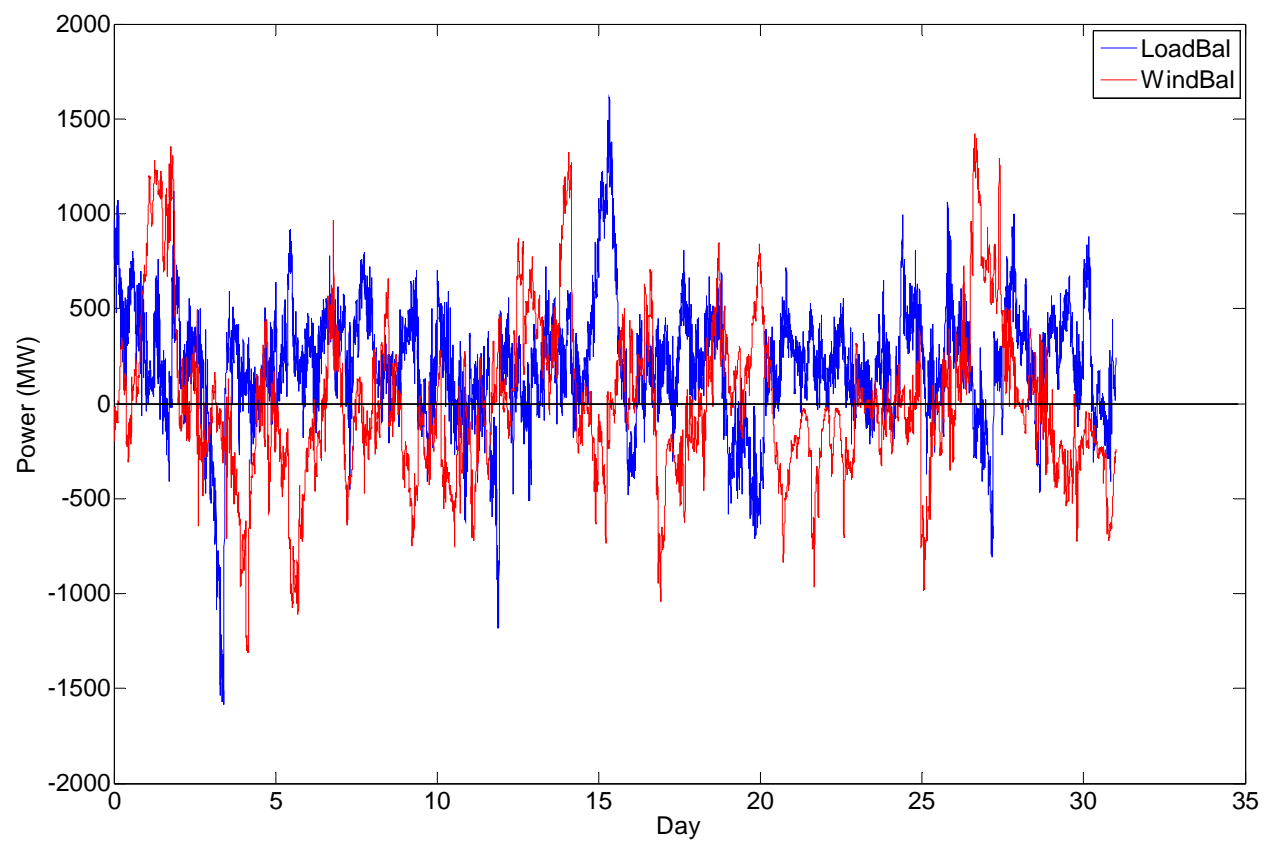

Figure 8.30. One Month Balancing Requirements Caused by Load and Wind Respectively for RMPP

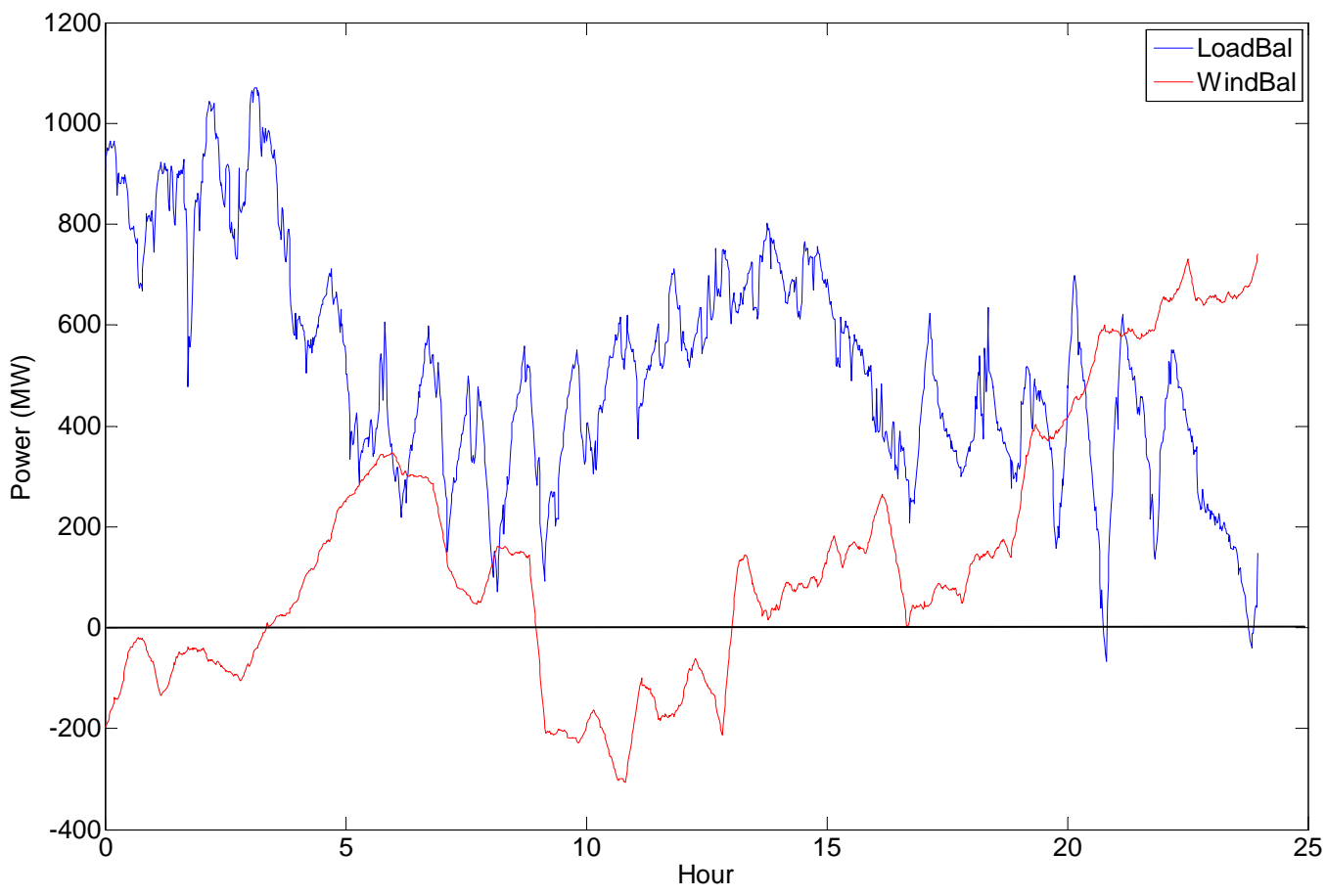

Figure 8.31. One Typical Day Balancing Requirements Caused by Load and Wind Respectively for RMPP 


\subsubsection{Energy and Power Requirements}

Extensive systems modeling were performed to estimate the power and energy capacity requirements to meet the future balancing needs. Each technology and technology group required careful simulation that incorporated the specific technical features of a technology, as well as the interaction with each other if more than one technology was modeled. The results of the simulations were a pairing of power (GW) capacity, and energy (GWh) capacity requirements to meet future balancing needs. A detailed discussion of how the technologies were dispatched individually or within an ensemble of other technologies can be found in Appendix B.

Table 8.19 and Figures 8.32 and 8.33 show the results of energy and power requirements for the scenarios in the RMPA. It should be noted that the capacity requirements or the minimal size of the battery is based on $100 \%$ DOD of the battery. This means that the size of the energy storage is fully utilized. The storage will be cycled from fully charged to fully discharged. As will be discussed, there are good economic reasons for upsizing the battery to a DOD of less than $100 \%$ to improve the life of the battery. For instance, a battery with a DOD of $50 \%$ only uses its energy storage capability to $50 \%$. Significant simulation efforts were performed to determine the minimal capacity (power and energy rating) for the various technology options. The key driver that set the size of the technology was specific operational constraints that force the technology to be operated in a certain way, for instance, the limited change modes and the changeover delay of the pumped hydro and compressed air technologies.

Table 8.19. Power and Energy Requirements for Each Scenario for RMPP. Note: The energy capacity (GWh) for the batteries is nominated at a DOD of $100 \%$.

\begin{tabular}{clcc}
\hline Case & \multicolumn{1}{c}{ Technology } & GW & GWh \\
\hline C1 & Combustion turbine & 0.68 & - \\
C2 & Na-S & 0.67 & 0.22 \\
C3 & Li-ion & 0.67 & 0.22 \\
C4 & Flywheel & 0.68 & 0.22 \\
C5 & CAES & 1.34 & 7.33 \\
& Na-S & 0.44 & 0.05 \\
C6 & Flow battery & 0.67 & 0.22 \\
C7 & PH multiple modes & 0.67 & 0.23 \\
& 4-min waiting period, Na-S & 0.33 & 0.11 \\
C8 & PH 2 modes & 1.34 & 7.37 \\
& 4-min waiting period, Na-S & 0.36 & 0.03 \\
C9 & DR & 2.37 & - \\
\hline
\end{tabular}

The size for the combustion turbine is set by the requirements for generation increment, not the sum of increment and decrement. This is based on the notion that the existing combustion turbine capacity is operating at the zero balancing point already and would be able to provide generation decrements.

Notice that there are differences in the sizes of storage (GW and GWh) required for the different cases of studies (see Table 8.20). These differences are due to the efficiencies and operation strategies of the storage technologies. The GW and GWh differences in cases C2 to C4 and C6 are only due to 
difference in storage efficiency. The GW and GWh difference in case C7 is due to storage efficiency and due to the need of an additional storage technology (Na-S) to provide balancing during the 4-minute waiting period needed to change between charging and discharging mode (pumping and generation). The large GW and GWh difference in case C5 and C8 with respect to the rest of the cases is mainly because of the restriction in operation assumed; a restriction of only two mode changes (charging to discharging or discharging to charging) is assumed causing a large increase in size requirement (GW and GWh). Details of operation strategies for each technology can be found in Appendix B.

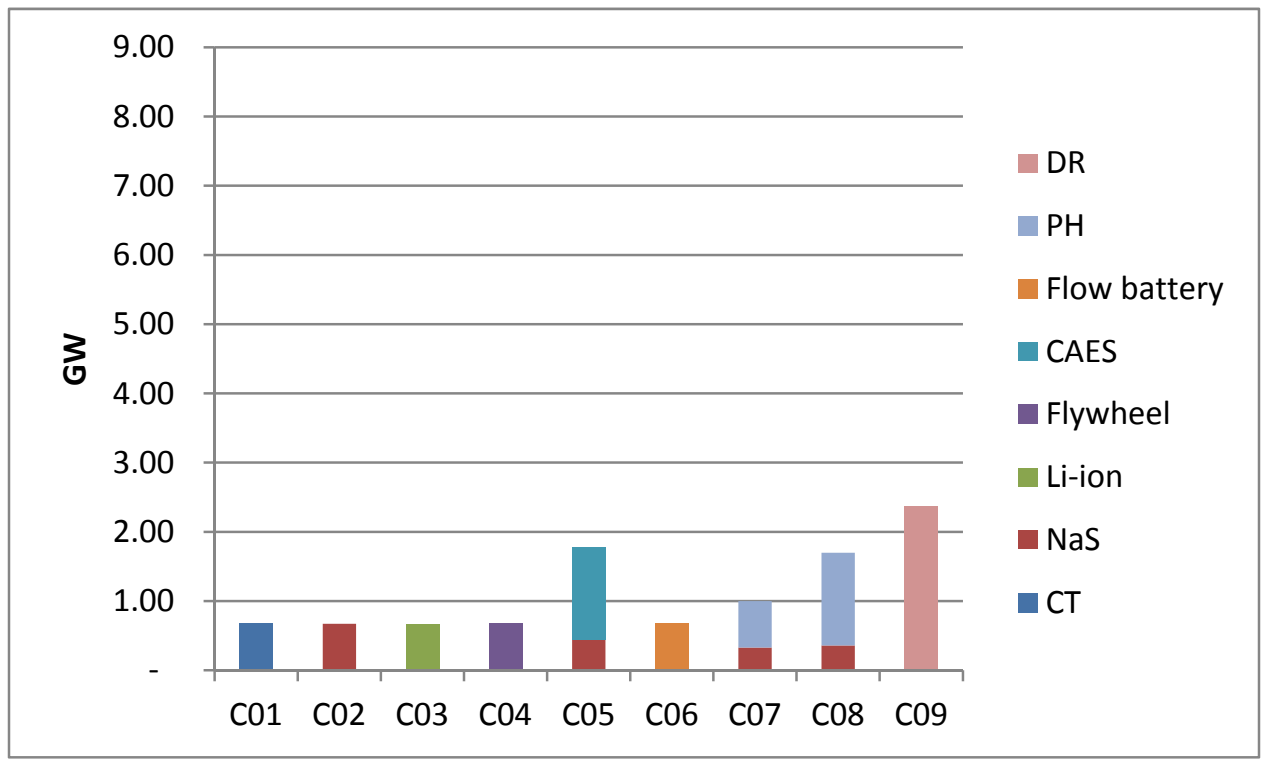

Figure 8.32. Power Requirements for all the Technologies to Meet Balancing Signal for RMPP

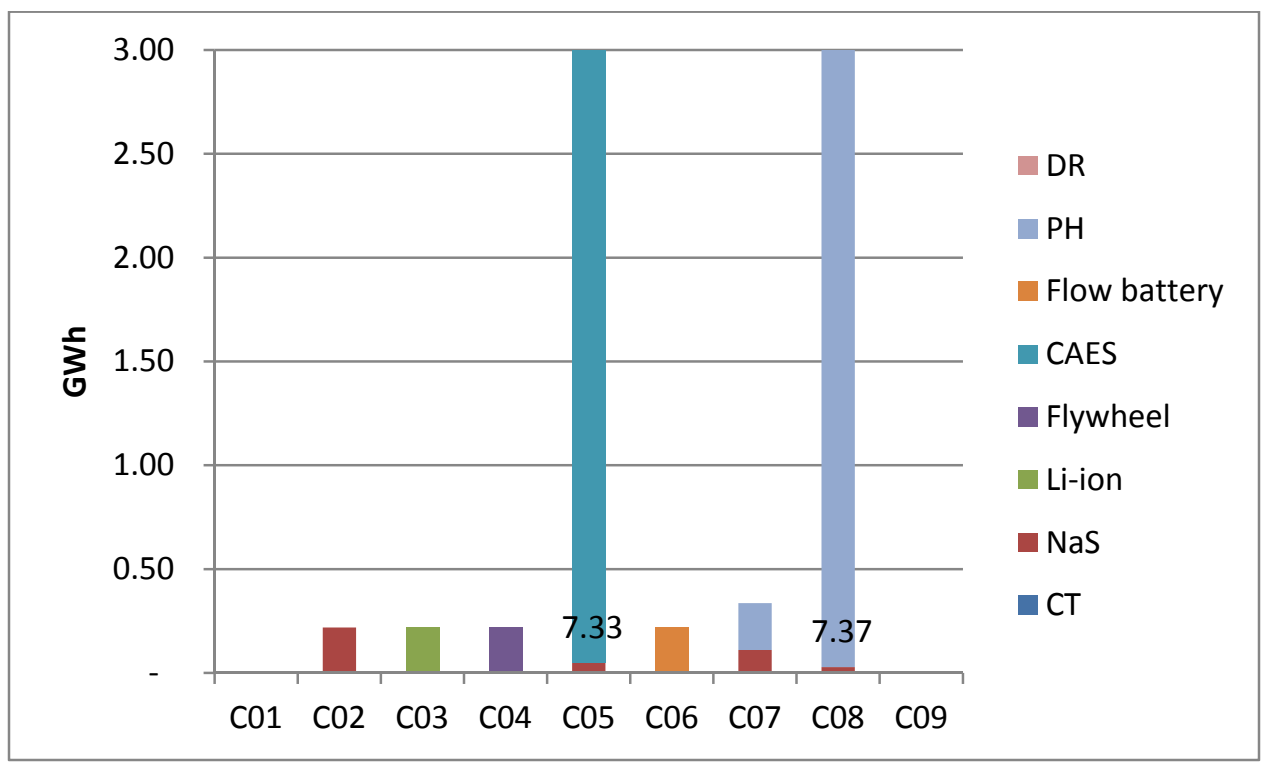

Figure 8.33. Energy Requirements for Storage Technologies to Meet Balancing Signal for RMPP 
Table 8.20 and Figures 8.34 and 8.35 show the results of energy and power requirements for the scenarios in the RMPA area, considering only the additional wind generation and load expected between 2011 and 2012. These are the requirements for only additional balancing assuming that the 2011 level of balancing is still provided by existing resources.

Table 8.20. Power and Energy Requirements for Each Scenario due to 2011-2020 Additional Wind and Load for RMPP. Note: The energy capacity (GWh) for the batteries is nominated at a DOD of $100 \%$.

\begin{tabular}{clcc}
\hline Case & \multicolumn{1}{c}{ Technology } & GW & GWh \\
\hline C1 & Combustion turbine & 0.52 & - \\
C2 & Na-S & 0.51 & 0.19 \\
C3 & Li-ion & 0.51 & 0.19 \\
C4 & Flywheel & 0.51 & 0.18 \\
C5 & CAES & 0.99 & 6.16 \\
& Na-S & 0.23 & 0.02 \\
C6 & Flow battery & 0.51 & 0.19 \\
C7 & PH multiple modes & 0.51 & 0.18 \\
& 4-min waiting period, Na-S & 0.17 & 0.03 \\
C8 & PH 2 modes & 0.99 & 6.19 \\
& 4-min waiting period, Na-S & 0.18 & 0.01 \\
C9 & DR & 1.73 & - \\
\hline
\end{tabular}

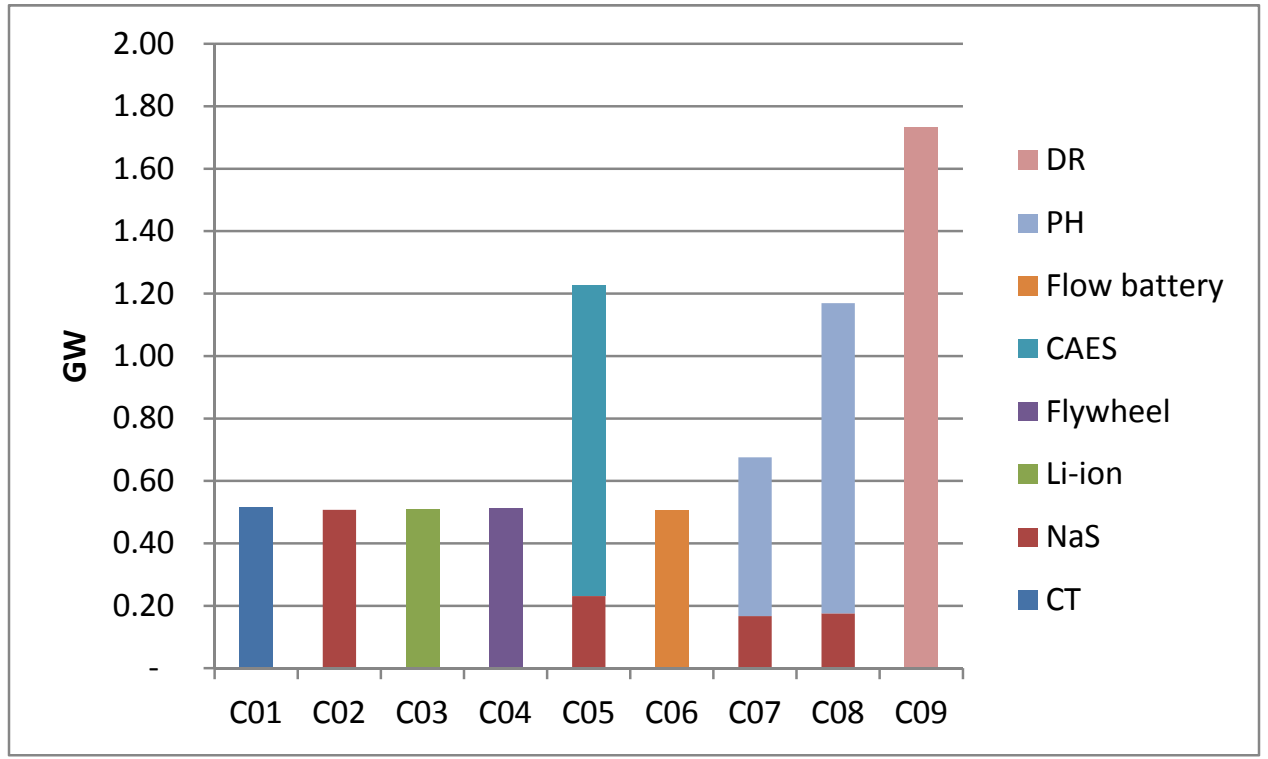

Figure 8.34. Power Requirements for all the Technologies to Meet Balancing Signal due to 2011-2020 Additional Wind and Load for RMPP 


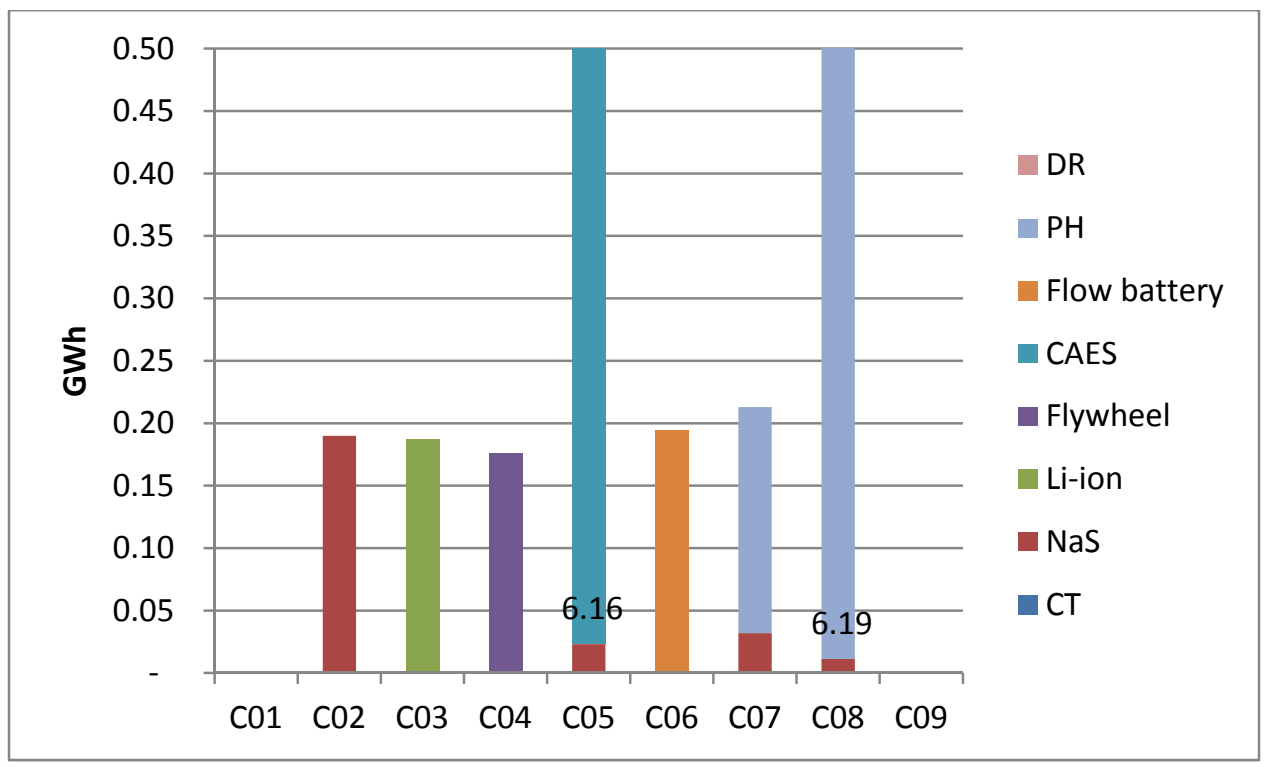

Figure 8.35. Energy Requirements for Storage Technologies to Meet Balancing Signal due to 2011-2020 Additional Wind and Load for RMPP

\subsubsection{Life-Cycle Cost Analysis}

The results of the economic analysis for the RMPA are presented in Table 8.21 and Figure 8.36. These results represent the base or reference case for the nine technologies defined in Section 5.1. The values presented in Table 8.21 represent the present value of the stream of capital, O\&M, fuel, and emissions costs over a 50-year time horizon, using the 2020 price values presented in Section 3.3 discounted at $8.0 \%$.

Table 8.21. Economic Analysis Results - RMPA (2020 Prices)

\begin{tabular}{crrrrr}
\hline Case & Capital & Fuel & O\&M & Emissions & Total \\
\hline 1 & 1,990 & 380 & 156 & 150 & $\mathbf{2 , 6 7 8}$ \\
2 & 766 & 57 & 98 & 22 & $\mathbf{9 4 4}$ \\
3 & 1,233 & 51 & 97 & 20 & $\mathbf{1 , 4 0 1}$ \\
4 & 899 & 24 & 201 & 10 & $\mathbf{1 , 1 3 4}$ \\
5 & 2,290 & 508 & 318 & 201 & $\mathbf{3 , 3 1 7}$ \\
6 & 1,873 & 66 & 91 & 26 & $\mathbf{2 , 0 5 6}$ \\
7 & 2,176 & 51 & 101 & 20 & $\mathbf{2 , 3 4 8}$ \\
8 & 4,183 & 139 & 251 & 55 & $\mathbf{4 , 6 2 7}$ \\
9 & 2,277 & - & - & - & $\mathbf{2 , 2 7 7}$ \\
\hline
\end{tabular}

Case 2, which employs Na-S batteries plus CC plants, is the least cost alternative at $\$ 0.9$ billion. Case 4, which consists of flywheels plus CC plants, represents the second least cost alternative with costs estimated at $\$ 1.1$ billion or $20.1 \%$ higher than those estimated for Case 2. The costs associated with the DR-only case (Case 9) are more than twice as expensive as those estimated for the two aforementioned 
cases, registering at $\$ 2.3$ billion. The CAES case (Case 5) is also more expensive with estimated costs of $\$ 3.3$ billion. In the predominantly PH case with two mode changes per day (Case 8), total costs are estimated at $\$ 4.6$ billion. Total costs under Case 6 , redox flow batteries plus CC plants, are estimated at $\$ 2.1$ billion.

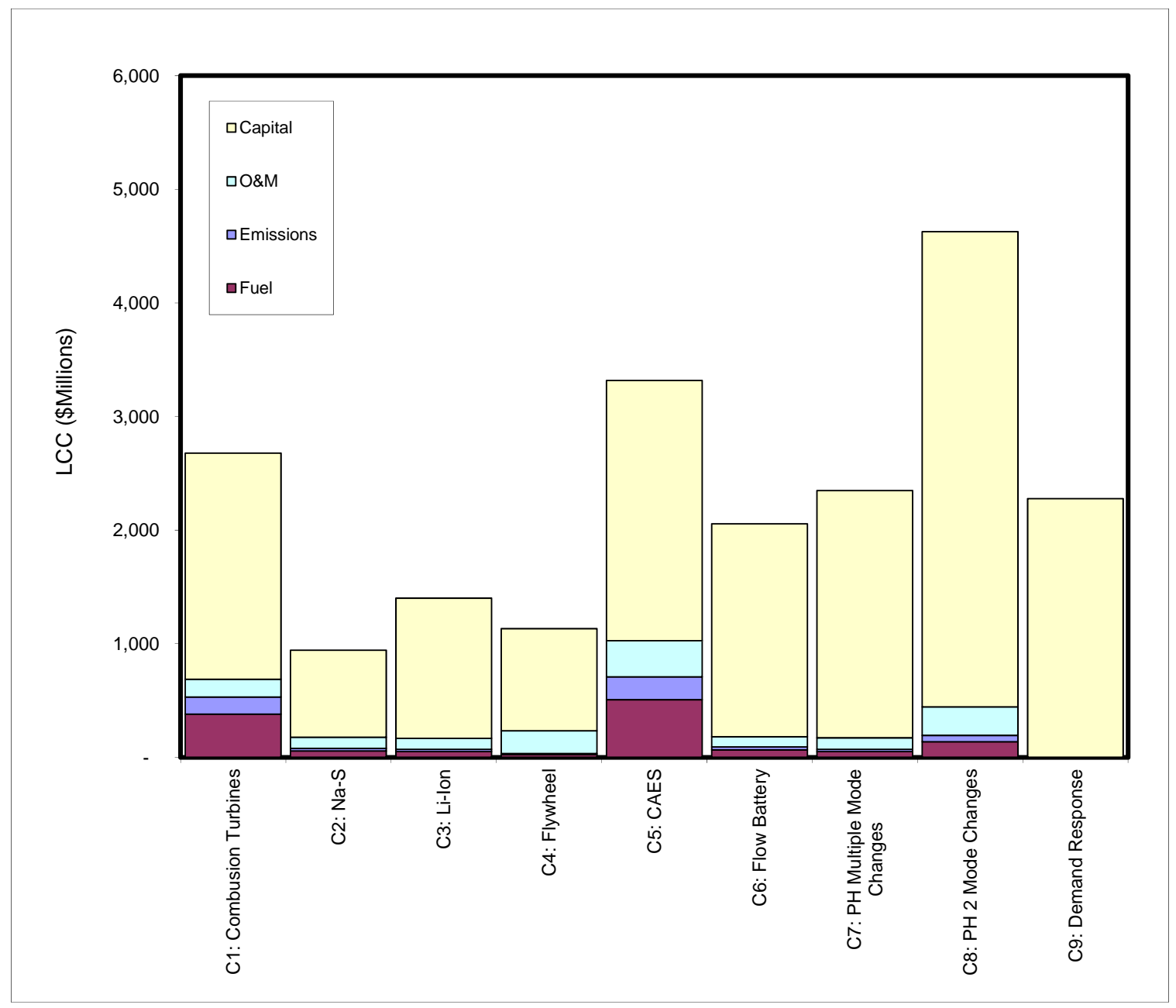

Figure 8.36. Scenario LCC Estimates for RMPP

\subsubsection{Arbitrage}

Arbitrage analysis was not performed in the RMPA.

\subsubsection{Sensitivity Analysis}

The accuracy of wind and load forecast are main factors which affect the balancing requirements. In the analysis, we investigated the impacts of wind forecast accuracy on balancing requirements and required energy storage size by assuming the wind forecast to be more accurate than current forecast accuracy. Because the mean value of wind forecast is close to zero, standard deviation of wind forecast error is used as metric to evaluate the accuracy of wind forecast. When the accuracy of wind forecast decreases which means the standard deviation of wind forecast error becomes greater, the balancing 
requirements are very likely to increase as shown in Figure 8.37 and Figure 8.38. The forecast accuracy has higher impacts if the wind adoption level in the region is higher. So greater changes are observed for regions with more wind capacity installed such as NWPP and CAMX. If the standard deviation of the wind forecast error is zero, this means wind has a perfect forecast. The balancing requirements are mainly caused by load forecast error, load within an hour variations and wind within an hour variations.

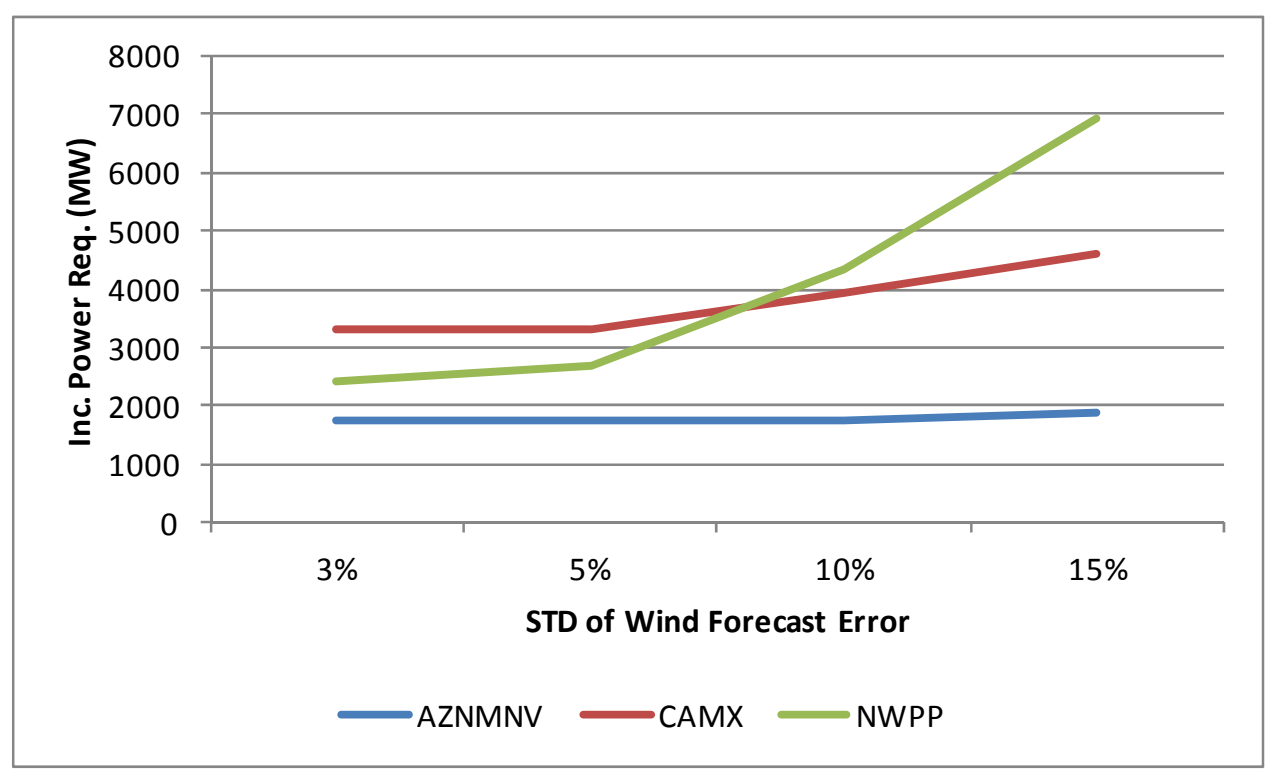

Figure 8.37. Impacts of Wind Forecast Error on Incremental Power Requirements

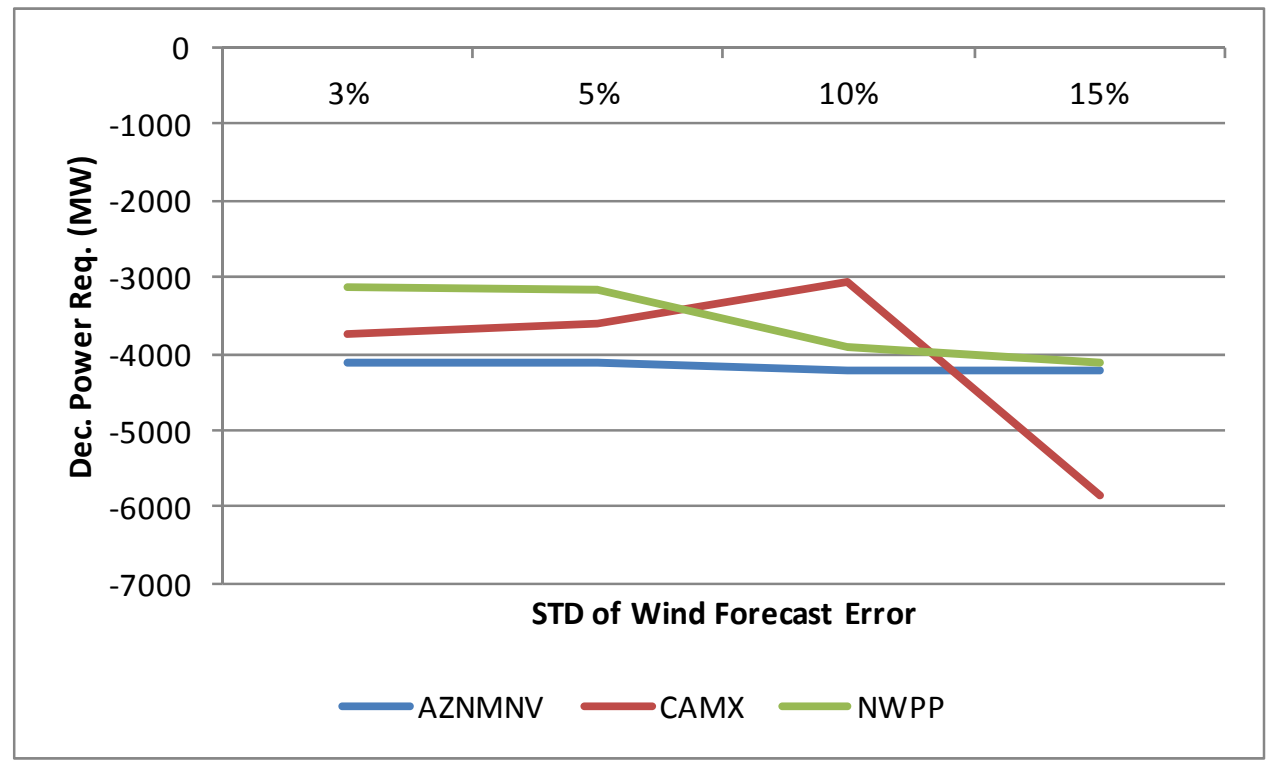

Figure 8.38. Impacts of Wind Forecast Error on Decremental Power Requirements

The balancing requirements assessment is based on a probabilistic method; 20 simulations have been done for NWPP to assess the convergence of the method. The results are shown in Table 8.22. The balancing requirements based on 20 runs are close to the results obtained based on one run which is shown in Table 8.22. 
Table 8.22. Total Balancing Requirements of NWPP Based on 20 Simulations

\begin{tabular}{lccc}
\hline & Power (MW) & Ramp Rate (MW/min) & Duration (min) \\
\hline NWPP 20 & 3775 & 183 & 30 \\
Runs & -3545 & -205 & \\
\hline
\end{tabular}




\subsection{Summary and Conclusions}

This National Assessment for Grid-Connected Energy Storage estimated the total and additional or incremental balancing requirements for four NERC WECC sub-regions for a projected 2020 grid scenario with a total of $24 \mathrm{GW}$ of wind capacity to meet a hypothetical RPS requirements of $20 \%$ across the WECC. The total balancing requirements were defined as the flexible grid assets necessary to accommodate all of the entire variability associated with renewable generation and load variabilities for a 2020 grid scenario. The additional balancing requirements define the grid asset necessary to accommodate the additional variability in load growth and the presumed $14 \mathrm{GW}$ of wind capacity additions in the WECC between 2011 and 2020. Within the groups of total and additional balancing requirements there are 2 subcategories: 1 ) total requirements including the entire spectrum of variability and 2) intra-hour balancing requirements (variabilities with a cycle period of up to 2 hours).

\subsection{Balancing Requirements}

Each sub-region was assumed to be a consolidated balancing area. The balancing-up requirements (generation increment, assigned a positive sign) and the balancing-down (generation decrement) ${ }^{1}$ are presented in Table 9.1. for both the total and intra-hour requirements.

Table 9.1. Total and Intra-Hour Balancing Requirements for every NERC Region in WECC in 2020

\begin{tabular}{ccccc}
\hline & \multicolumn{2}{c}{ Total Balancing Requirements } & \multicolumn{2}{c}{ Intra-Hour Balancing Requirements } \\
\cline { 2 - 5 } & $\begin{array}{c}\text { Power } \\
(\mathrm{MW})\end{array}$ & $\begin{array}{c}\text { Ramp Rate } \\
(\mathrm{MW} / \mathrm{min})\end{array}$ & $\begin{array}{c}\text { Power } \\
(\mathrm{MW})\end{array}$ & $\begin{array}{c}\text { Ramp Rate } \\
(\mathrm{MW} / \mathrm{min})\end{array}$ \\
\hline \multirow{2}{*}{ AZNM } & +2740 & 353 & +1090 & 294 \\
& -3550 & & -1220 & 229 \\
CAMX & +4130 & 215 & +1790 & 197 \\
& -2920 & & -2400 & 117 \\
NWPP & +3430 & 186 & +1700 & -2020 \\
& -2730 & & +670 & -670 \\
\hline
\end{tabular}

The results indicate that the total balancing requirements span a spectrum of frequencies, from minute-to-minute variability (intra-hour balancing ${ }^{2}$ ) to those indicating cycles over several hours (interhour balancing. The intra-hour balancing requirements were extracted from the total balancing requirements and are also presented in Table 9.1. The intra-hour balancing requirements are generally smaller than the total requirements because they represent only the higher frequency components of the entire balancing signal, but their ramp rate requirements are comparable. This analysis focused primarily on the intra-hour balancing market because the sharp ramp rates in this market are of significant concern

\footnotetext{
${ }^{1}$ These estimates are based on BPA's customary 99.5\% probability bound.

2 The intra-hour balancing requirement was filtered out of the total balancing signal, is smaller than the total requirements, and tends to have similar ramp rate requirements.
} 
to grid operators and furthermore, inter-hourly balancing (over several hours) can be accommodated in redispatch of generators in hour-ahead energy markets.

Table 9.2. Additional Intra-Hour and Total Intra-Hour Balancing Requirements for WECC Sub-Regions

\begin{tabular}{|c|c|c|c|c|c|c|c|}
\hline & $\begin{array}{c}\text { Additional } \\
\text { Balancing } \\
\text { Power } \\
\text { Required } \\
\text { (MW) }\end{array}$ & $\begin{array}{l}\text { Total } \\
\text { Balancing } \\
\text { Power } \\
\text { Required } \\
\text { (MW) }\end{array}$ & $\begin{array}{c}\text { Total } \\
\text { Balancing } \\
\text { Power } \\
\text { Required as a } \\
\text { Percentage of } \\
\text { Peak Load } \\
(\%) \\
\end{array}$ & $\begin{array}{c}\text { Marginal } \\
\text { Balancing } \\
\text { Power } \\
\text { Required as a } \\
\text { Percentage } \\
\text { Wind Capacity } \\
(\%) \\
\end{array}$ & $\begin{array}{l}\text { Existing } \\
\text { Wind } \\
\text { Capacity } \\
\text { (MW) }\end{array}$ & $\begin{array}{l}\text { Additional } \\
\text { Wind } \\
\text { Capacity } \\
\text { (MW) }\end{array}$ & $\begin{array}{c}\text { Total } \\
\text { Wind } \\
\text { Capacity } \\
\text { in } 2020 \\
\text { (MW) }\end{array}$ \\
\hline AZNM & 210 & 1220 & 4 & 22 & 390 & 970 & 1360 \\
\hline CAMX & 530 & 2400 & 4 & 13 & 2430 & 4110 & 6540 \\
\hline NWPP & 280 & 2020 & 3 & 7 & 5560 & 4200 & 9760 \\
\hline RMPA & 510 & 670 & 5 & 10 & 1170 & 5160 & 6330 \\
\hline Total & 1530 & 6320 & & & 9550 & 14440 & 23990 \\
\hline
\end{tabular}

This study indicates that the future total intra-hour balancing requirements to address both load and renewable variability are expected to range between $3 \%$ and $5 \%$ of the peak load in a given region. Furthermore, on the margin for every additional unit of wind capacity power, approximately 0.07 to 0.22 units of intra-hour balancing need to be added.

These values most likely under-estimate the size of the balancing market and the additional generation or storage power needed because a simplifying assumption was made in the analysis that the current individual BA are consolidated to one single, large balancing area within each sub-region. This consolidating assumption takes advantages of load diversity and renewable generation diversity within a sub-region.

\subsection{Market Size for Energy Storage for Balancing Services}

The assessment estimated the size requirements for energy storage capacity to meet the total and additional intra-hour balancing requirements as summarized in Figure 9.2. 


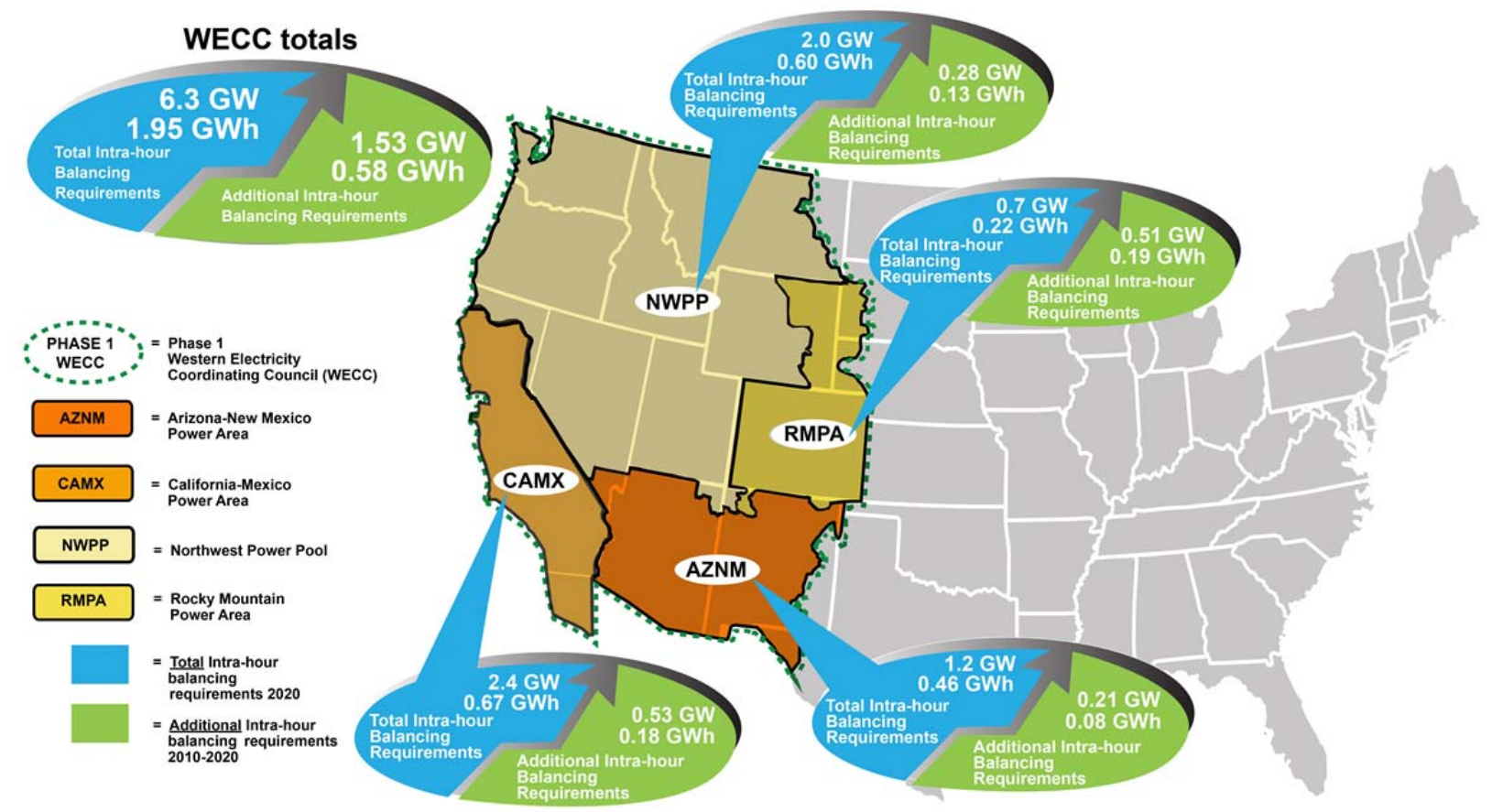

Figure 9.1. Size Requirements for Storage Technologies to Meet the Total and Additional Intra-Hour Balancing Services for a 2020 Grid with 20\% RPS

\subsection{Life-Cycle-Cost Analysis of Various Technology Options to Meet Future Balancing Requirements}

A detailed LCC analysis was performed that sought the optimal cost combinations of generation and storage technologies to meet the total intra-hour balancing requirements over a 50-year lifetime. Our analysis evaluates the nine case of different technology options listed in Table 9.3.

Cost components considered include capital, O\&M costs, as well as fuel prices and typical prices for criteria emissions. The carbon dioxide $\left(\mathrm{CO}_{2}\right)$ emissions were valued at a cost of $\$ 45 /$ ton $\mathrm{CO}_{2}$. It was assumed that all of the estimated balancing requirements will be met with new investments. Significant emphasis was placed on reviewing the literature regarding the characterization of storage and generation options for grid applications, and on choosing plausible and defensible cost performance characteristics of the technologies under consideration.

This study revealed several insights into the competitiveness of the nine cases shown in Table 9.3 of different technology options when optimizing for LCCs. First, the results of the economic analysis for all four WECC sub-regions indicate that of the nine cases examined in this report, Case 2, which employs Na-S batteries plus CC natural gas plants, is expected to be the most economical alternative in 2020. For the NWPP, the 50-year LCC for Case 2 when using forecast 2020 prices is $\$ 2.8$ billion. The next least cost alternative is Case 4 (flywheels plus CC plants) at $\$ 3.3$ billion, followed by Case 3 (Li-ion batteries plus CC plants) at $\$ 4.3$ billion, and Case 6 (redox flow batteries plus CC plants) at $\$ 6.2$ million. While Na-S batteries (Case 2) appears to be the most cost-effective option for balancing in both 2011 and 2020, this analysis assumes that Na-S batteries will be available in the required energy to rated power ratio of 
$\sim 1: 1$. Currently, this ratio is about seven, thus requiring a battery seven times the size selected in this study. This is the main reason Na-S batteries are not competitive with CT at present.

Table 9.3. Definition of Technology Cases

\begin{tabular}{|c|c|c|}
\hline Case & Technology & Comments \\
\hline $\mathrm{C} 1$ & $\begin{array}{l}\text { Combustion } \\
\text { turbine }\end{array}$ & Conventional technology considered as the reference case. \\
\hline $\mathrm{C} 2$ & $\mathrm{Na}-\mathrm{S}$ & Sodium sulfur battery only. \\
\hline C3 & Li-ion & Lithium-ion battery only. \\
\hline C4 & Flywheel & Flywheel only. \\
\hline C5 & $\begin{array}{l}\text { CAES with } 2 \\
\text { mode changes }\end{array}$ & $\begin{array}{l}\text { CAES with a 7-minute waiting period for mode changes (compression-generation } \\
\text { and vice versa). Balancing services will be provided during compression mode at } \\
\text { night ( } 8 \mathrm{pm}-8 \mathrm{am}) \text { and during generation mode during the day ( } 8 \mathrm{am}-8 \mathrm{pm}) \text {. Na-S } \\
\text { battery is assumed to make up operations during } 7 \text { minute waiting period. }\end{array}$ \\
\hline C6 & Flow battery & Flow battery only. \\
\hline C7 & $\begin{array}{l}\text { PH with multiple } \\
\text { mode changes }\end{array}$ & $\begin{array}{l}\text { PH with a 4-minute waiting period for mode changes (pumping-generation and vice } \\
\text { versa). This machine allows multiple mode changes during the day. Na-S battery is } \\
\text { assumed to make up operations during } 4 \text { minute waiting period. }\end{array}$ \\
\hline $\mathrm{C} 8$ & $\begin{array}{l}\text { PH with } 2 \text { mode } \\
\text { changes }\end{array}$ & $\begin{array}{l}\text { Same as (C7), except only two mode changes. Balancing services will be provided } \\
\text { during pumping mode at night ( } 8 \mathrm{pm}-8 \mathrm{am}) \text { and during generation mode during the } \\
\text { day ( } 8 \mathrm{am}-8 \mathrm{pm}) \text {. Na-S battery is assumed to make up operations during } 4 \text { minute } \\
\text { waiting period. }\end{array}$ \\
\hline C9 & DR & $\begin{array}{l}\text { Demand response only. This assumes that balancing services will be provided as a } \\
\text { load. Only considered is PHEV charging at home and work. Resources are } \\
\text { expressed in MW of DR capacity as well as in numbers of PHEV with demand } \\
\text { response capability. }\end{array}$ \\
\hline
\end{tabular}

Flywheels (Case 4) appear most cost-effective for both 2011 and 2020 (unless Na-S systems can be manufactured at energy to rated power ratio of 1 by 2020). For 2011, CAES (Case 5) and pumped hydro with multiple mode changes (Case 7), while costlier than flywheels (Case 4), are competitive with CTs (Case 1), while Li-ion systems (Case 3) are slightly costlier than CTs (Case 1). For 2020, all energy storage options are competitive with CTs (Case 1), except CAES (Case 5) and PH 2-mode (Case 8). Even at the high end of the capital cost estimates, in 2020, Li-ion (Case 3) and flywheels (Case 4) are expected to be cost-competitive with CTs (Case 1), while flow batteries (Case 6) are expected to be only barely costlier.

In nearly all cases, the costs associated with the energy storage options are lower than those estimated for the combustion turbine case ${ }^{1}$ (Case 1), particularly with respect to fuel and emissions costs. For the NWPP, costs for Case 1 (CT) are estimated at $\$ 7.1$ billion, while the pumped hydro cases (Case 7 and Case 8), which vary based on the assumed number of mode changes per day, are estimated to cost between $\$ 6.8$ and $\$ 13.2$ billion. Under the current scenarios, capital costs drive the outcome of the analysis and the CT and pumped hydro with their corresponding high capital costs do not perform well.

\footnotetext{
${ }^{1}$ Natural gas used for combustion turbine is assumed to be $\$ 4.94$ per MMBTU in 2011 escalated at 3.2\% over the 50-year analysis time horizon.
} 
The findings of this analysis suggest that both options appear ill-suited for providing balancing services. For example, pumped hydro with its large reservoir is underutilized in this analysis.

Figure 9.2 presents the results of the LCC analysis and the effects of capital, O\&M, emissions, and fuel costs on the total LCC for each case, as applied in the NWPP. Note that costs are also presented per MW to meet intra-hour balancing requirements for the NWPP. As noted in the figure, the bar chart uses 2020 cost assumptions while the results using 2011 price data are identified using the brackets added to the figure. For example, the LCC for the Na-S case (Case 2) is \$2.8 billion when using 2020 prices but rises to $\$ 3.9$ billion when using present 2011 prices.

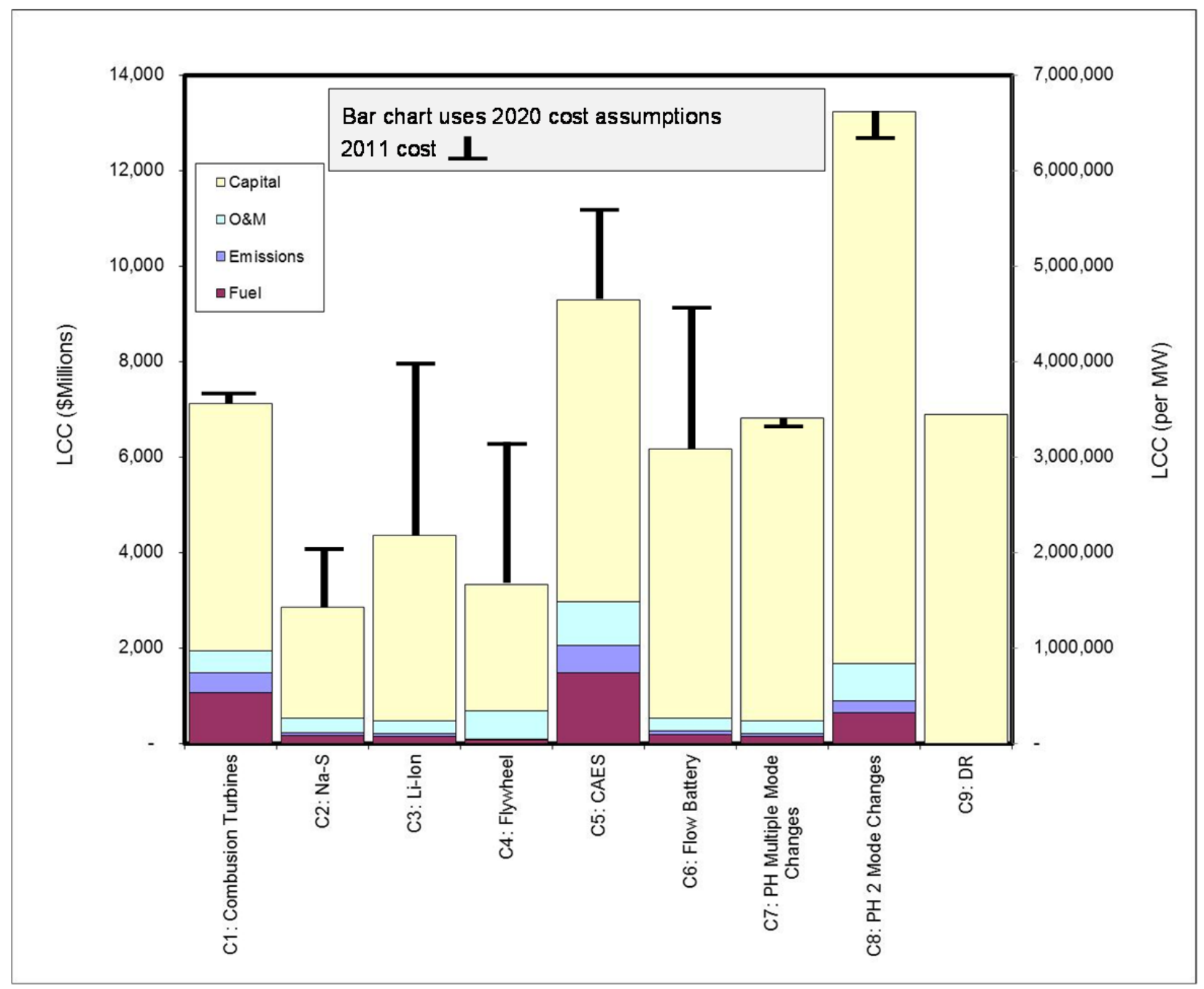

Figure 9.2. Scenario LCC Estimates

The technical and cost uncertainty regarding energy storage technologies is evaluated in TRLs and MRLs. TRLs assigned to the energy storage technologies are as low as six for redox flow batteries and seven for Na-S batteries, Li-ion batteries, and flywheels. A TRL of six indicates that a prototype system has been verified while a TRL of seven indicates that an integrated pilot system has been demonstrated. Conversely, CT received a TRL of nine, which indicates that the system is proven and ready for full 
commercial deployment. The MRL for flywheels and redox flow batteries is five indicating that the manufacturing process is under development. Na-S and Li-ion batteries received MRLs of six indicating that a critical manufacturing process for utility-scale systems has been prototyped. CTs received an MRL rating of 10 indicating that full rate production has been demonstrated and lean production practices are in place.

\subsection{Hybrid Storage Systems}

Additionally, this analysis investigated cases in which the balancing service was provided by combinations of two storage or generation technologies. The balancing signal was divided into "slow" and "fast" components. These balancing components are satisfied by two storage or generation technologies with different technical and economic characteristics. The LCC methodology was applied to different shares of the combination of technologies to determine the split with the highest cost effectiveness. The main finding is that technology share comprised primarily of the lower-cost technology was always the most cost-effective. The only exceptions to this were for combinations of Li-ion and DR technologies, and combinations of pumped hydro with multiple mode change and flywheels. This result stems from the non-linearity in these two combination cases. For the Li-ion and DR combination case under the 2011 price scenario, the least cost technology share was $60 \%$ DR and $40 \% \mathrm{Li}$-ion in most regions as shown in Figure 9.3. There was a non-linearity originated by the availability of DR. For the pumped hydro with multiple mode changes plus flywheels combination case, the least cost technology share was 60\% PH and 40\% flywheel for the CAMX area and under the 2011 price scenario. The non-linearity in this case is stemmed from the waiting period between mode changes of pumped hydro. The non-linearity influences the technology share outcome when the costs of the two technologies are comparable.

To quantify the arbitrage value of energy storage, a cost effectiveness approach was developed that contrasted the annual revenue requirements from the capital expenditure to the revenue potential from arbitrage. The revenue potential analysis is based on a production cost approach using PROMOD IV ${ }^{1}$ from Ventyx as an analysis tool. PROMOD is a production cost software that solves security constrained unit commitment and dispatch problems in power systems at either the zonal or nodal transmission level.

\footnotetext{
${ }^{1}$ PROMOD IV is an Energy Planning and Analytics Software developed by Ventyx http://www.ventyx.com/analytics/promod.asp.
} 


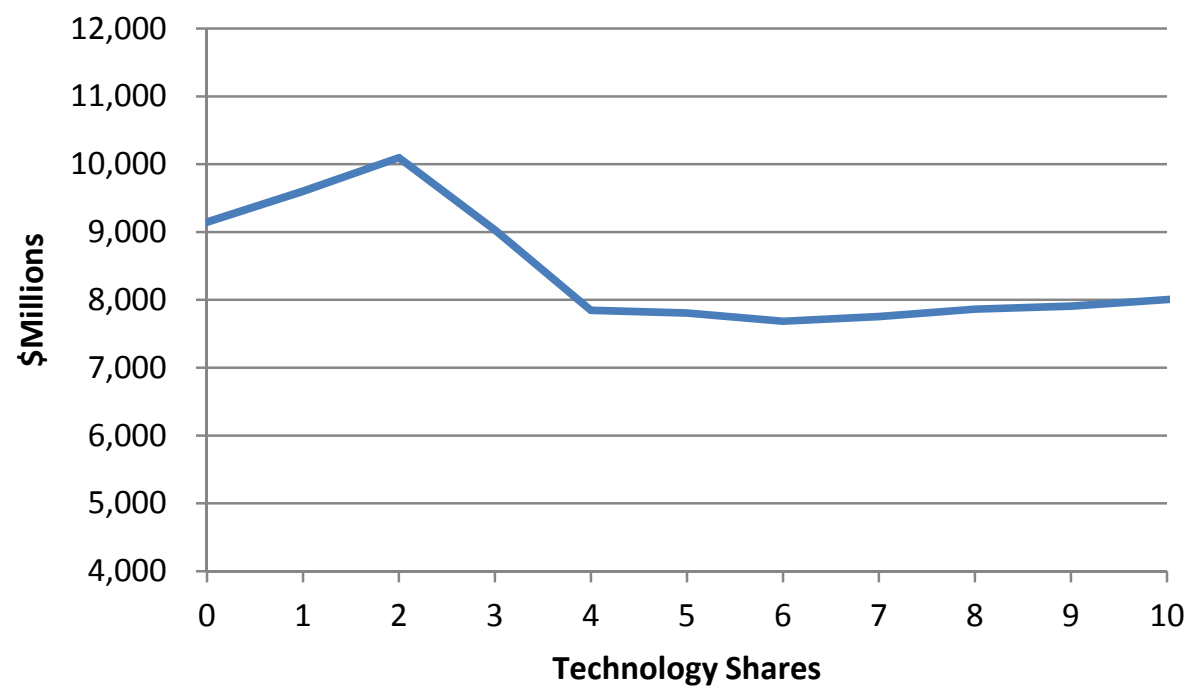

Figure 9.3. Total 50-Year LCCs for Li-Ion +DR Technology Shares for 2011 Cost Assumptions. Optimal combination (technology share 6) only present in two cases under 2011 cost assumptions.

\subsection{Energy Arbitrage Opportunities}

The revenue potential of arbitrage was determined by identifying constraints in the system and determining the LMP differential for hours throughout the year along those congested paths. Simulations were then carried out for different energy storage sizes for the NWPP and CAMX. The results of the WECC simulation are presented in Table ES.4. Arbitrage revenues in the WECC grow from $\$ 4.3$ million annually at $243 \mathrm{MW}$ of installed capacity to $\$ 99.1$ million at 9,712 MW.

Annual costs are also presented in Table ES.4, demonstrating that arbitrage revenue expectations fall short of the revenue requirements necessary for cost recovery. This general finding applies to each scenario presented in this report. Thus, it would take either a significant increase in the peak- to off-peak LMP differential for extended periods of time or reductions in energy storage capital costs for energy arbitrage as designed in this analysis to break even. Under the current projections for the WECC, even the $243 \mathrm{MW}$ scenario fails to yield profits when applied to the most profitable hours of the year. This result supports the conclusion that at a 30\% reserve margin, the WECC region is not sufficiently congested for energy storage to become cost-effective when used to provide only arbitrage services.

While the findings of this analysis indicate that profits from energy arbitrage are insufficient to achieve capital cost recovery, it is important to note that there are several other services that could be supplied by energy storage technologies that were excluded from this assessment. These services include load following, transmission and distribution upgrade deferral, power quality enhancements, electricity service reliability, and additional reserve capacity. Thus, revenues from the energy storage technologies are not fully realized due to the limited focus of the analysis. Additional research is therefore necessary to examine the full revenue potential of energy storage used in multiple applications. 
Table 9.4. Annualized Revenue and Capital Costs for Na-S Batteries, Li-Ion Batteries, and Pumped Hydro

\begin{tabular}{|c|c|c|c|c|c|}
\hline \multicolumn{2}{|c|}{ Storage Size } & \multirow[t]{2}{*}{$\begin{array}{c}\text { Annual } \\
\text { Revenue } \\
\text { (in \$mill.) }\end{array}$} & \multicolumn{3}{|c|}{ Annualized Costs (in \$ mill.) } \\
\hline MWh & MW & & Pumped Hydro & $\mathrm{Na}-\mathrm{S}$ & Li-Ion \\
\hline 2,400 & 240 & $\$ 4.3$ & $\$ 49$ & $\$ 111$ & $\$ 217$ \\
\hline 4,900 & 490 & $\$ 8.6$ & $\$ 99$ & $\$ 221$ & $\$ 435$ \\
\hline 9,700 & 970 & $\$ 16.7$ & \$197 & $\$ 443$ & $\$ 870$ \\
\hline 12,100 & 1,210 & $\$ 20.5$ & $\$ 246$ & $\$ 553$ & $\$ 1,087$ \\
\hline 24,300 & 2,430 & $\$ 37.9$ & $\$ 493$ & $\$ 1,106$ & $\$ 2,175$ \\
\hline 36,400 & 3,640 & $\$ 52.3$ & $\$ 739$ & $\$ 1,660$ & $\$ 3,262$ \\
\hline 48,600 & 4,860 & $\$ 66.0$ & $\$ 986$ & $\$ 2,213$ & $\$ 4,350$ \\
\hline 60,700 & 6,070 & $\$ 75.2$ & $\$ 1,232$ & $\$ 2,766$ & $\$ 5,437$ \\
\hline 97,100 & 9,710 & $\$ 99.1$ & $\$ 1,971$ & $\$ 4,426$ & $\$ 8,699$ \\
\hline
\end{tabular}

\subsection{Overall Conclusions}

Phase I results provide crucial insights into the potential market size for energy storage from a national perspective. The following conclusions can be drawn from this analysis:

1. The total amount of power capacity for a 20\% RPS scenario in the WECC would require a total intrahour balancing capacity of approximately $\sim 6.32 \mathrm{GW}$. The total market size was estimated for the WECC by NERC sub-regions based on the potential for energy storage in the high-value balancing market. The energy capacity, if provided by energy storage, would be approximately $2.0 \mathrm{GWh}$, or a storage that could provide power at rated capacity for about 20 minutes. The addition intra-hour balancing capacity that is required to accommodate the variability due to capacity addition in wind technology and load growth from 2011-2020 was estimated to be $1.53 \mathrm{GW}$. If these additional balancing services were provided by new energy storage technology, the energy capacity would be about $0.58 \mathrm{GWh}$, or a storage capable of providing electricity at the rated power capacity for about 20 minutes.

2. The regional distribution of balancing requirements within the WECC is driven by load forecasting wind prediction errors. Because of the non-homogeneous distribution of the loads and wind across the WECC region, the balancing requirements increase with load and wind capacity. NWPP and California were the two major regions with significant intra-hour balancing requirements.

3. Various technologies compete for the growing balancing market opportunities, not only energy storage, but also DR. The base case technology is a gas-fueled CT, which may be attractive particularly under low cost gas projections for the next decades. The LCC analysis for intra-hour service indicated that Na-S, flywheel storage technologies, and DR are under current cost estimates are already cost-competitive (lowest LCC). Li-ion will follow under cost reduction assumption for the 2020 timeframe.

4. LCC results are strictly applicable for intra-hour balancing services with an average cycle time of about 20-30 minutes. As the application requires longer cycle times with higher energy capacity, 
capital costs and production cost of conventional generators will be different, all affecting the LCC results and the relative cost competitiveness.

5. Energy arbitrage alone is insufficient to provide sufficient revenues to make new energy storage installations economically viable even in congested paths such as transfer into Southern California and interchange at the California-Oregon border. Although this result was based on the production cost modeling that estimates cost differential between peak and off-peak, not market price differentials, which tends to be higher than the cost differentials, the frequency and duration of transmission congestions were simply not sufficient to make energy storage technologies a viable business proposition as an energy product. It will require other value streams such as a capacity payments that are valued more highly that the average energy value to augment the revenue stream for storage capacities. Potentially, with sufficiently large duration of energy storage beyond 4 hours, capital deferment in the bulkpower system and at the substation could provide additional revenues such that longer duration storage could be economically viable.

6. The hybrid energy storage system analysis did not show very compelling tradeoff between slower cycling and faster cycling technologies. In all cases the time resolution used (minute-by-minute) did not show sufficiently sharp transients such that ramp limits affected use and selection of some technologies. As a consequence, all of the optimal cost pairings of two technologies were determined solely on price, and, as such, the most prevalent results indicated a winner-take-all solution, in which the optimal pairing to technology suggested only one (namely the lower-cost) technology. This suggests that hybridizing storage technologies will only be meaningful if there are a wide spectrum of cycles expected with sharp transients with sub-one-minute time resolution, which this analysis did not expose. 



\subsection{References}

Active Power. 2011. Available at http://www.activepower.com/no_cache/solutions/whitepapers/. Accessed December 22, 2011.

Alstom. 2009. “Alstom Signs €178 Million Variable Speed Pumped-Storage Hydro Contract.” Press Release October 28, 2009. Accessed March 24, 2010 at http://www.alstom.com/pr_corp_v2/2009/corp/61513.EN.php?languageId=EN\&dir=/pr_corp_v2/2009/co rp/\&idRubriqueCourante=23132\&cookie=true (undated webpage).

Altair Nanotechnologies. 2008. “Advanced Batteries Supply Ancillary Services.” POWER, December 2008. Accessed March 24, 2010 at www.powermag.com (undated webpage).

Barnett B, D Ofer, C McCoy, Y Yang, T Rhodes, B Oh, M Hastbacka, J Rempel, and S Sririramulu. 2009. “PHEV Battery Cost Assessment.” U.S. Department of Energy Annual Merit Review, May 19, 2009, Washington D.C.

Beacon Power. 2010. "Beacon Power and NYSERDA Sign \$2 Million Contract in Support of Stephentown Flywheel Plant.” Accessed December 1, 2011 at http://investors.beaconpower.com/releasedetail.cfm?ReleaseID=560549.

Bindner H, C Ekman, O Gehrke, and F Isleifsson. 2010. “Characterization of Vanadium Flow Battery,” Riso Report Riso-R-1753(EN) October 2010.'

BINE Informationsdienst. 2007. “Compressed air energy storage power plants.” projektinfo 05/07. Accessed September 28, 2011 at http://www.bine.info/en/hauptnavigation/publications/publikation/druckluftspeicherkraftwerke/?artikel=277.

Bolund B. 2007. “Flywheel Energy and Power Storage Systems.” Renewable and Sustainable Energy Reviews 11:235-259.

Boyes J. Energy Storage Tutorial. Sandia National Laboratories, Albuquerque, New Mexico. Accessed March 24, 2010 at http://www.netl.doe.gov/technologies/coalpower/fuelcells/seca/tutorial/TutorialIII_files/TutorialIII.pdf (undated webpage).

Butler P, Miller JL, and Taylor PA. 2002. Energy Storage Opportunities Analysis Phase II Final Report. SAND2002-1314, May 2002, Sandia National Laboratories, Albuquerque, New Mexico.

CAISO - California Independent System Operator. 2009. Discussion Paper Renewable Integration: Market and Product Review. CAISO, July 2009.

CAISO - California Independent System Operator. 2010. Integration of Renewable Resources:

Technical Appendices for California ISO Renewable Integration Studies, Version 1, Draft 1, October 11, 2010. 
Cavallo A. 2007. "Controllable and affordable utility-scale electricity from intermittent wind resources and CAES.” Energy 32:120-127.

Cavallo A. 2011. E-mail to Vilayanur Viswanathan, received September 16, 2011.

Chiao E. 2011. “Amber Kinetics Flywheel Energy Storage Demonstration.” 2011 DOE-OE Annual Merit Review, October 20-21, San Diego, California.

Chintawar PS. 2010. E-mail message from Prashant S. Chintawar (BASF) to V.V. Viswanathan. “Li-ion Battery Cost,” February 3, 2010.

Colella WG, MZ Jacobson, and DM Golden. 2005. "Switching to a U.S. Hydrogen Fuel Cell Vehicle Fleet: The Resultant Change in Energy Use, Emissions, and Global Warming Gases.” Journal of Power Sources 150:150-181.

Corey GP, LE Stoddard, and RM Kerschen. 2002. Boulder City Battery Energy Storage Feasibility Study. Sandia Report SAND2002-0751, Sandia National Laboratories, Albuquerque, New Mexico.

Daniel R. 2008. CAES Strategic Needs. CAES Conference \& Workshop, Columbia University, New York. October 21.

Denholm P, E Ela, B Kirby, and M Milligan. 2010. The Tole of Energy Storage with Renewable Electricity Generation. Technical Report. NREL/TP-6A2-47187, National Renewable Energy Laboratory, Golden, Colorado.

Divya KC and J Østergaard. 2009. "Battery Energy Storage Technology for Power Systems.” Electric Power Systems Research 79(4):511-520.

Doane J, R O’Toole, R Chamberlain, P Bos, and P Maycock. 1976. The Cost of Energy from UtilityOwned Solar Electric Systems: A Required Revenue Methodology for ERDA/EPRI Evaluations. California Institute of Technology, Pasadena, California.

DOD - U.S. Department of Defense. 2010. U.S. Department of Defense Manufacturing Readiness Levels. Deskbook, 3 January 2010 Draft, Washington, D.C.

DOE - U.S. Department of Energy. 2009. U.S. Department of Energy Technology Readiness Assessment Guide. DOE G 413.3-4 10-12-09, Washington, D.C.

DOE - U.S. Department of Energy, Office of Electricity Delivery and Energy Reliability. 2011. Energy Storage. Program Planning Document, U.S. Department of Energy, Office of Electricity Delivery \& Energy Reliability, Washington, D.C.

DOE/EIA. 2011. The Electricity Market Module of the National Energy Modeling System. Model Documentation Report. DOE/EIA-M068(2011), Energy Information Administration, Washington, D.C.

DOE/EIA. 2008. Annual Energy Outlook 2008. Energy Information Administration, Washington, D.C. DOE/EIA. 2009. Annual Energy Outlook 2009. Energy Information Administration, Washington, D.C. 
DOE/EIA. 2010a. Annual Energy Outlook 2010. Energy Information Administration, Washington, D.C.

DOE/EIA. 2010b. Annual Energy Outlook 2011. Energy Information Administration, Washington, D.C.

DOT - U.S. Department of Transportation. 2003. Highlights of the 2001 National Household Travel Survey. BTS03-05, Table A-8, U.S. Department of Transportation, Bureau of Transportation Statistics, Washington, D.C.

Drury E, P Denholm, and R Sioshansi. 2011. “The Value of Compressed Air Energy Storage in Energy and Reserve Markets.” Energy 36:4959-4973.

EAC - Electricity Advisory Committee. 2008. Bottling Electricity: Storage as a Strategic Tool for Managing Variability and Capacity Concerns in the Modern Grid. Prepared by Energetics Incorporated for the Electricity Advisory Committee.

Eckroad S. 2004. EPRI-DOE Handbook Supplement. "Energy Storage for Grid Connected Wind Generation Applications,” EPRI, Palo Alto, California, and the U.S. Department of Energy, Washington, D.C. 2004.1008703.

Eckroad S. 2007. Vanadium Redox Flow Batteries: An In-Depth Analysis. EPRI, 1014836, Palo Alto, California.

Energystoragenews.com. 2010. "Vanadium Redox Flow Batteries for Large Scale Storage of Electricity.” Accessed October 3, 2011 at http://www.energystoragenews.com/Vanadium\%20Redox\%20Flow\%20Batteries.htm.

EPA - U.S. Environmental Protection Agency. 1995. "Compilation of Air Pollutant Emission Factors, Volume 1: Stationary Point and Area Sources.” AP 42, Fifth Edition, U.S. Environmental Protection Agency, Washington, D.C.

EPRI - Electric Power Research Institute. 2009. Assessment of Achievable Potential from Energy Efficiency and Demand Response Programs in the U.S. Palo Alto, California.

EPRI - Electric Power Research Institute. 2009. Economic and Greenhouse Gas Emission Assessment of Utilizing Energy Storage Systems in ERCOT. EPRI 1017284, November 2009.

Evolution Markets. 2010. Jan/Feb 2010. NOx Market Monthly Update (Annual NOx Allowances).

Figueiredo FC and PC Flynn. 2006. “Using Diurnal Power Price to Configure Pumped Storage.” IEEE Transactions on Energy Conversion EC 21(3):804-809.

Eyer J and G Corey. 2010. "Energy Storage for the Electricity Grid: Benefits and Market Potential Assessment Guide.” SAND2010-0815, Sandia National Laboratories, Albuquerque, New Mexico.

Eyer JM, JJ Iannucci, and GP Corey. 2004. "Energy Storage Benefits and Market Analysis Handbook, A Study for the DOE Energy Storage Systems Program,” SAND2004-6177, December 2004. 
FERC, NOPR. 2011. Frequency Regulation Compensation in the Organized Wholesale Power Markets. Docket Nos. RM11-7-00, Federal Energy Regulatory Commission, February 17, 2011, Washington, D.C.

First Hydro Company. 2009. "Welcome to First Hydro Company.” International Power, June 4, 2009.

Fodstad LA. Pumped Storage. Staraft Energy AS, Oslo, Norway.

GE Energy. 2004. E to Provide Equipment for 1,000-Megawatt Hydropower Station in China. Press Release March 15, 2004. Accessed March 24, 2010 at http://www.gepower.com/about/press/en/2004_press/031504a.htm (undated webpage).

Geadah A. 2009. Introducing Pumped Storage in Lebanon: Towards a Prospective National Master Plan. International Seminar on River Basin Management and Co-operation in the Euro-Mediterranean Region, October 6-9, 2009, Beirut, Lebanon.

Gray B. 2009. Grid Scale Energy Storage: Linchpin Technology for our Clean and Secure Energy Future Environmental Energy Technologies Division Seminars, E.O. Lawrence Berkeley National Laboratory, March 17. Accessed Nov 30, 2011 at http://eetd-seminars.lbl.gov/seminar/grid-scale-energystorage-linchpin-technology-our-clean-and-secure-energy-future.

Gray B. 2011. Telephone conversation with Bill Gray of Velkess, Inc. on September 27, 2011.

Grazzini G and A Millazzo. 2008. “Thermodynamic analysis of CAES/TES systems for renewable energy plants.” Renewable Energy 44:1998-2006.

Greenberg D, I Krepchin, and K Kamm. "Big Batteries Blooming.” Power. Accessed March 24, 2010 at http://www.powermag.com/print/environmental/Big-batteries-blooming_1042.html (undated webpage).

Greenblatt JB. 2005. "Using gas turbines to enhance the value of wind power.” $C C J$ 1Q:34-8.

Gyuk IP and S Eckroad. 2003. EPRI-DOE Handbook of Energy Storage for Transmission and Distribution Applications, 1001834 Final Report, December 2003. Prepared by Technology Insights and EPRI PEAC Corporation for the U.S. Department of Energy, Washington D.C., and Electric Power Research Institute, Inc., Palo Alto, California.

Gyuk IP and S Eckroad. 2004. EPRI-DOE Handbook Supplement of Energy Storage for Grid Connected Wind Generation Applications, 1008703 Technical Update, December 2004. Prepared by Technology Insights and EPRI PEAC Corporation for the U.S. Department of Energy, Washington D.C., and Electric Power Research Institute, Inc., Palo Alto, California.

Hazel J. 2010. E-mail message from John Hazel (Lithium Technology Corporation) to VV Viswanathan (PNNL), “Li-ion Battery Cost,” February 3, 2010.

Henson W. 2008. “Optimal battery/ultracapacitor storage combination.” Journal of Power Sources 179(1):417-423, ISSN 0378-7753, 10.1016/j.jpowsour.2007.

Herman D. 2003. Comparison of Storage Technologies for Distributed Resource Applications. Electric Power Research Institute, Palo Alto, California, 2003.1007301. 
Hirst E. 2002. Integrating Wind Energy with the BPA Power System: Preliminary Study. Available at http://www.bpa.gov/power/pgc/wind/Wind_Integration_Study_09-2002.pdf.

Hoffman P, H Miller, and J Kerth. 2008. "McIntosh CAES Experience NYSERDA-Columbia University Compressed Air Energy Workshop.” CAES Conference \& Workshop, Columbia University, October 21-22.

Howell D. 2009. “Annual Merit Review: Energy Storage R\&D Overview.” U.S. Department of Energy, Energy Efficiency and Renewable Energy.

Jacobson MZ, WG Colella, and DM Golden. 2005. "Cleaning the Air and Improving Health with Hydrogen Fuel Cell Vehicles.” Science 308:1901-1905.

Jenkinson P. 2005. “Introduction.” International Power First Hydro Analysis Conference, July 2005.

Joerissen L, J Garche, Ch Fabjan, and G Tomazic. 2004. "Possible use of vanadium redox-flow batteries for energy storage in small grids and stand-alone photovoltaic systems.” J. Power Sources 127:98-104.

Jossen A and DU Sauer. 2006. “Advances in Redox-Flow batteries.” First International Renewable Energy Storage Conference, October 30-31, Gelsenkirchen, Germany.

Dragoon K and M Milligan. 2003. "Assessing Wind Integration Costs with Dispatch Models: A Case Study of PacifiCorp.” WindPower 2003.

Kaizuka T and T Sasaki. 2001. "Evaluation of control maintaining electric power quality by use of rechargeable battery system.” In IEEE Power Engineering Society Winter Meeting, 2001, vol 1, pp. 88-93.

Kamibayashi M, DK Nichols, and T Oshima. 2002. "Development Update for the NAS Battery." Transmission and Distribution Conference and Exhibition 2002: Asia Pacific, IEEE/PES 3:1664-1668. DOI:10.1109/TDC.2002.1176850.

Kannurpatti AR. Dupont. Telephone conversation on July 14, 2011.

Kear G, AA Shah, FC Walsh. 2011. "Development of the all-vanadium redox flow battery for energy storage: a review of technological, financial and policy aspects.” Int. J. Energy Res. 2011, DOI:10.1002/er.1863.

KEMA - Keuring Electrotechnisch Materieel Arnhem. 2010. Research Evaluation of Wind Generation, Solar Generation and Storage Impact on the California Grid. June 2010, CEC-500-2010-010.

KEMA- Keuring Electrotechnisch Materieel Arnhem. 2008. "Summary of KEMA Validation Report: Two Megawatt Advanced Lithium-ion BESS Successfully Demonstrates Potential for Utility Applications.”

Kishinevsky Y. 2006. “Long Island Bus Sodium Sulfur Battery Storage Project.” U.S. DOE Peer Review, Washington, D.C. 
Lamont A. 2004. Improving the Value of Wind Energy Generation Through Back-up Generation and Energy Storage. CWEC-2003-005, Table 4. Prepared for California Wind Energy Collaborative by Lawrence Livermore National Laboratory.

Lazarewicz M. 2011. Telephone conversation of Viswanathan with M Lazarewicz on September 12, 2011.

Lemofouet S and A Rufer. 2006. “A Hybrid Energy Storage System Based on Compressed Air and Supercapacitors with Maximum Efficiency Point Tracking (MEPT).” Industrial Electronics, IEEE Transactions 53(4):1105-1115.

Li L, K Soowhan, W Wang, M Vijayakumar, Z Nie, B Chen, J Zhang, G Xia, J Hu, G Graff, J Liu, and Z Yang. 2011. "A Stable Vanadium Redox-Flow Battery with High Energy Density for Large-Scale Energy Storage.” Adv. Energy Mater. 1:394-400.

Lipman TE, R Ramos, and DM Kammen. 2005. An Assessment of Battery and Hydrogen Energy Storage Systems Integrated with Wind Energy Resources in California . PIER Final Project Report CEC-500-2005-136, Table 1. Prepared by University of California, Berkeley for California Energy Commission, Public Interest Energy Research Program.

Liu H and J Jiang. 2007. "Flywheel energy storage - An upswing technology for energy sustainability." Energy and Buildings 39(5):599-604.

Lu N and DJ Hammerstrom. 2006. "Design Considerations for Frequency Responsive Grid Friendly Appliances." In 2005/2006 IEEE/PES Transmission and Distribution Conference and Exhibition, May 21-24, 2006, Dallas, Texas, pp. 647-652, Institute of Electrical and Electronics Engineers, Piscataway, New Jersey.

Lucas G and H Miller. 2010. Dresser-Rand's SMARTCAES Compressed Air Energy Storage Solution, 2nd Compressed Air Energy Storage Conference \& Workshop, Columbia University, October 20 \& 21, 2010.

Lukic SM, SG Wirasingha, F Rodriguez, J Cao, and A Emadi. 2006. "Power Management of an Ultracapacitor/Battery Hybrid Energy Storage System in an HEV.” 2006 IEEE Vehicle Power and Propulsion Conference. VPPC '06, pp. 1-6, September 6-8, 2006.

Macchi E and G Lozza, 1987. "A study of Thermodynamic performance of CAES plants, including unsteady effects.” Presented at the Gas Turbine Conference and Exhibition, Anaheim, California, May 31-June 4, 1987.

Makarov Y, M Kintner-Meyer, P Du, C Jin, and H Illian. 2010a. "Using DFT for Cycle Analysis of Energy Storage Capacity Requirement to Accommodate High Penetration of Renewable Resources in the WECC System.” IEEE PES Innovative Smart Grid Technologies Conference, January 19-21, 2010, Gaithersburg, Maryland. 
Makarov YV, JF Reyes Spindola, NA Samaan, R Diao, and RP Hafen. 2010b. "Wind and Load Forecast Error Model for Multiple Geographically Distributed Forecasts." In Proceedings of the 9th International Workshop on Large-Scale Integration of Wind Power into Power Systems as well as on Transmission Networks for Offshore Wind Power Plants, Quebec City, Quebec, Canada, October 18-19, p. Paper No. w151, Energynautics GmbH, Langen, Germany.

Makarov Y, S Lu, B McManus, and J Pease. 2008a. “The Future Impact of Wind on BPA Power System Ancillary Services.” Transmission and Distribution Conference and Exposition, T\&D, IEEE/PES, pp. 1-5.

Makarov YV, J Ma, S Lu, and T Nugyen. 2008b. Assessing the Value of Regulation Resources Based on Their Time Response Characteristics. PNNL-17632, Pacific Northwest National Laboratory, Richland, Washington.

Miller R. 2010. E-mail messages from Rick Miller (Renewable Energy Services to V.V. Viswanathan (PNNL), “Pumped Hydro Cost and Performance,” January 26, 29, February 1, and 22, 2010.

Miller H. 2011. E-mail message from Harry Miller to Vilayanur Viswanathan, “Attention Harry Miller,” October 4, 2011.

Nakhamkin M, B Kraft, and C Moran. 2010. Advanced Second Generation of CAES Technology $180 \mathrm{MW}, 310 \mathrm{MW}$ and $450 \mathrm{MW}$ CAES Plants. 2nd CAES Conference \& Workshop, Columbia University, October 20-21.

Nakhamkin M, M Chiruvolu, and C Daniel. 2007. “Available CAES Plant Concepts.” Available at www.espcinc.com/library/PowerGen_2007_paper.pdf. Accessed November 1, 2011.

Nakhamkin M, M Chiruvolu, M Patel, S Byrd, and R Schainker. 2009. "Second Generation of CAES Technology: Performance, Operations, Economics, Renewable Load Management, Green Energy.” POWER-GEN International, December 8-10, 2009, Las Vegas Convention Center, Las Vegas, Nevada.

Nakhamkin M. 2008. Second Generation of the CAES Technology. CAES Conference \& Workshop, Columbia University, October 21-22.

Nelson PA, DJ Santini, and J Barnes. 2009. “Factors Determining the Manufacturing Costs of LithiumIon Batteries for PHEVs.” EVS24 International Battery, Hybrid and Fuel Cell Electric Vehicle Symposium, May 13-16, 2009, Stavanger, Norway.

Nexight. 2010. Electric Power Industry Needs for Grid-Scale Storage Roadmap. (Draft), September 20, 2010. Results of a workshop organized by Sandia National Laboratories, Albuquerque, New Mexico.

NGK Insulators, LTD. Accessed at http://www.ngk.co.jp/english/index.html (undated webpage).

NHC - The Nevada Hydro Company, Inc. 2007. "Lake Elsinore Advanced Pump Storage FERC Project Number 11858 and Talega-Escondido/Valley-Serrano 500-kV Interconnect (LEAPS/TE-VS).” CEC Joint Committee Workshop, Transmission Corridors, May 14, 2007. 
Northwest Power and Conservation Council. 2010. "Sixth Northwest Conservation and Electric Power Plan.” Portland, Oregon.Nourai A. 2007. Installation of the First Distributed Energy Storage System (DESS) at American Electric Power (AEP): A Study for the DOE Energy Storage Systems Program. SAND2007-3580, Sandia National Laboratories, Albuquerque, New Mexico.

Nourai A. 2009. "Why and How Electric Vehicle Li-ion Batteries are Penetrating the Utility Market.” EV Li-ion Battery Forum, September 2-3, 2009, Shanghai, China.

Nourai A. 2010. E-mail message from Ali Nourai (American Electric Power) to V.V. Viswanathan (PNNL), “Na-S Battery System Cost,” February 2, 2010.

NREL - National Renewable Energy Laboratory. 2009. “About the Wind Integration Datasets.” Accessed March 24, 2010 at http://www.nrel.gov/wind/integrationdatasets/about.html (last updated November 24, 2009), p. 24 f 27.

NYISO -New York Independent System Operator. 2010. Proposed NYISO Installed Capacity Demand Curves for Capacity Years 2011/2012/2013/2014. Final Report, October 2010, New York Independent Systems Operator.

Pacini H and M Landauer. 2010. Phone conversation to Heidi Pacini (WECC), Mark Landauer (WECC). March 12, 2010.

Parker Hannifin Corp. 2010. SSD Drives Division. Available at http://www.ssddrives.com/usa/News/press_releases.php January 12, 2010 press release. Accessed March 26, 2010.

Pratt R, C Conner, E Richman, K Ritland, W Sandusky, and M Taylor. 1989. Description of Electric Energy Use in Single-Family Residences in the Pacific Northwest. End-use Load And Consumer Assessment Program (ELCAP), DOE/BP-13795-21, Bonneville Power Administration, Portland, Oregon.

Prodromidis A and FA Coutelieris. 2011. "Simulations of economical and technical feasibility of battery and flywheel hybrid energy storage systems in autonomous projects.” Renewable Energy 39(1):149-153.

Rahman S. 1990. "Economic Impact of Integrating Photovoltaics with Conventional Electric Utility Operation.” IEEE Transactions on Energy Conversion 5(3):422-428. DOI:10.1109/60.105264.

Rastler D, P Lemar, and S Price. 2007. Market Driven Distributed Energy Storage Requirements for Load Management Applications Technical Update 1014668. Prepared by Electric Power Research Institute, Resource Dynamics Corporation, and Energy Environmental Economics, Inc. for Electric Power Research Institute, Palo Alto, California.

Rastler D. 2010. Electricity Energy Storage Technology Options: A White Paper Primer on Applications, Costs and Benefits. EPRI 1020676, Final Report, December 2010, Electric Power Research Institute, Palo Alto, California.

Rastler D. 2011a. “Energy Storage Applications and Economics: Costs, Benefits, Revenue.” California Energy Commission IEPR Committee Workshop Energy Storage for Renewable Integration, Sacramento, California, April 28, 2011. 
Rastler D. 2011b. “MISO Energy Storage Study Phase 1 Report.” EPRI 1024489, Draft Report, November 2011, Electric Power Research Institute, Palo Alto, California.

Rastler D. 2012. “Benchmarking the Costs of Energy Storage Systems.” 22nd Annual ESA Meeting: Electricity Storage: Meeting the Challenge of an Evolving Grid, May 2-4, 2012, Washington D.C.

Ridge Energy Storage \& Grid Services L.P. 2005. The Economic Impact of CAES on Wind in TX, OK and NM. Final Report, June 27, 2005, Report for Texas State Energy Conservation Office.

Riedel G. 2010. E-mail message from Gary Riedel (Compact Power, Inc.) to V.V. Viswanathan (PNNL), “Li-ion Battery Cost,” February 2, 2010.

Ritterhausen J and M McDonagh. 2011. "Moving Energy Storage from Concept to Reality: Southern California Edison’s Approach to Evaluating Energy Storage.” White paper, May 202011.

Rounds R and GH Peek. 2008. Design \& Development of a 20-MW Flywheel-based Frequency Regulation Power Plant. Sandia Report SAND 2008-8229, Sandia National Laboratories, Albuquerque, New Mexico.

Rousseau A, N Shidore, R Carlson, and V Freyermuth. 2007. Research on PHEV Battery Requirements and Evaluation of Early Prototypes. Argonne National Laboratory, Chicago, Illinois. Accessed March 24, 2010 at http://www.transportation.anl.gov/pdfs/HV/434.pdf (undated webpage).

Rydh CJ. 1999. "Environmental assessment of vanadium redox and lead-acid batteries for stationary energy storage.” J. Power Sources 80:21-29.

Schainker RB, WJ Steeley, and V M-Wray. 2010. Insights from EPRI's CAES Economic Benefit-Cost Analyses. 2nd CAES Conference \& Workshop, Columbia University, October 20-21.

Schoenung S, J Eyer, J Iannucci, and S Horgan. 1996. “Energy Storage for a Competitive Power Market.” Annual Review of Energy and the Environment 21:347-370.

DOI:10.1146/annurev.energy.21.1.347.

Schoenung SM and WV Hassenzahl. 2003. Long vs. Short-Term Energy Storage Technology Analysis. SAND2003-2783, Sandia National Laboratories, Albuquerque, New Mexico.

Schoenung SM. 2001. Characteristics and Technologies for Long vs. Short-Term Energy Storage: A Study by the DOE Energy Storage Systems Program. SAND2001-0765, Sandia National Laboratories, Albuquerque, New Mexico.

Schoenung SM and J Eyer. 2008. Benefit/Cost Framework for Evaluating Modular Energy Storage. SAND2008-0978, February 2008, Sandia National Laboratories, Albuquerque, New Mexico.

Sibley L. 2011a. Available at www.tribologysystems.com. Accessed September 2, 2011.

Sibley L. 2011b. Telephone conversation with Lew Sibley, of Tribology Systems, Inc. on September 26, 2011. 
Sibley L. 2011c. E-mail received from Lew Sibley of Tribology Systems, Inc. on October 27, 2011.

Skyllas-Kazacos M. 2010. New Generation Vanadium Redox Battery. 2010 Electric Metals Conference, April 14, 2010.

Skyllas-Kazacos M. 2011. The Vanadium Redox Flow Battery: Science and Materials. Accessed October 31, 2001 at http://www.ccrhq.org/userfiles/file/Niche/Skyllas-Kazacosweb.ppt.pdf.

Spitzer F and G Penninger. 2008. "Pumped Storage Power Plants—Different Solutions for Improved Ancillary Services through Rapid Response to Power Needs.” HydroVision 2008, July 2008.

Staudt L. Undated. Electricity Storage: Windfarm and Industrial Applications. Centre for Renewable Energy, Dundalk Institute of Technology, Ireland. Accessed on October 26, 2011 at www.engineersireland.ie/sector_papers/EI_Storage_Presentation.pdf.

Steeley W. 2005. VRB Energy Storage for Voltage Stabilization. EPRI Report 1008434, March 2005, Electric Power Research Institute, Palo Alto, California.

Succar S and RH Williams. 2008. Compressed Air Energy Storage: Theory, Resources and Applications for Wind Power. Princeton Environmental Institute, Princeton University, April 8, 2008.

Sullivan P, W Short, and N Blair. 2008. Modeling the Benefits of Storage Technologies to Wind Power. Conference Paper NREL/CP-670-43510, June 2008, National Renewable Energy Laboratory, Golden, Colorado.

Taylor P. et al. 1999. A summary of the state-of-the-art of SMES, flywheel, and CAES. Sandia report SAND991854, July 1999, Sandia National Laboratories, Albuquerque, New Mexico.

Technology Insights. 2005. Overview of NAS Battery for Load Management. CEC Energy Storage Workshop, February 2005.

Tokuda N, T Kanno, T Hara, T Shigematsu, Y Tsutsui, A Ikeuchi, T Itou, and T. Kumamoto. 2000. "Development of a Redox Flow Battery System.” SEI Technical Review 50:88-93.

TEPPC - Transmission Expansion Planning Policy Committee. 2010. 2009 Study Program Results Report. Western Electricity Coordinating Council (WECC), Salt Lake City, Utah.

Tuffner F and M Kintner-Meyer. 2011. Using Electric Vehicles to Meet Balancing Requirements Associated with Wind Power. PNNL-20501, Pacific Northwest National Laboratory, Richland, Washington.

Ulibas O. Telephone conversation with Vilayanur Viswanathan of PNNL, December 22, 2011.

USCAR - United States Council for Automotive Research LLC. 2007. "USABC Final Cost Model 2007-05-23.” Available at http://www.uscar.org/guest/article_view.php?articles_id=143 (undated webpage). 
van der Linden S. 2006. "Bulk Energy Storage Potential in the USA, Current Developments and Future Prospects.” Energy 31(15):3446-3457.

Vanadiumsite. 2011. “Deeya Energy L-Cell Iron Chromium Flow Battery.” Accessed October 3, 2011 at http://www.vanadiumsite.com/vanadium-redox-l-cell-iron-chromium-flow-battery/.

Vfuel. 2011. “Company and Technology Information Sheet.” Accessed October 3, 2011 at www.vfuel.com.au/infosheet.pdf.

Vosen SR and JO Keller. 1999. "Hybrid energy storage systems for stand-alone electric power systems: optimization of system performance and cost through control strategies." International Journal of Hydrogen Energy 24(12):1139-1156, ISSN 0360-3199, 10.1016/S0360-3199(98)00175-X.

Walwalkar R, J Apt, and R Mancini. 2006. Economics of electric energy storage for energy arbitration and regulation in New York. Carnegie Mellon Electricity Industry Center Working Paper CEIC-06-04. Accessed October 26, 2011 at https://wpweb2.tepper.cmu.edu/ceic/pdfs/CEIC_06_04.pdf.

Western Electricity Coordinating Council. 2010. Transmission Expansion Planning Policy Committee. 2009 Study Program Results Report, January 26, 2010, WECC, Salt Lake City, Utah.

Yang Z, J Zhang, MC Kintner-Meyer, X Lu, D Choi, JP Lemmon, and J Liu. 2011. "Electrochemical Energy Storage for Green Grid.” Chem. Rev. 111(5):3577:613.

Zhang H. 2009. “R\&D Progress of Redox Flow Battery (RFB) for Energy Storage.” MS\&T 2009 Conference \& Exhibition.

Zhao P, H Zhang, H Zhou, J Chen, S Gao, and B Yi. 2006. "Characteristics and performance of 10kW class all-vanadium redox-flow battery stack.” J. Power Sources 162:1416-1420. 

Appendix A

\section{Detailed Technology Discussion}





\section{Appendix A}

\section{Detailed Technology Discussion}

\section{A.1 Sodium sulfur (Na-S) Battery}

\section{A.1.1 Battery Sizing Method}

Battery sizing depends on DOD, which depends on the number of cycles needed from the battery during its life. The only Na-S battery commercially available is from NGK Insulators, Ltd., which has an energy content of $\sim 6.8$ times the rated power (i.e., it has a storage capacity of 6.8 hours at rated power) (Nourai 2007). Hence, it is best suited for applications where a low DOD is desired for long life. As will be discussed in Section A.1.3, the batteries will be sized to cycles that are much shorter than 6.8 hours; they will be sized to $1 / 2-1$ hour. Na-S batteries with storage duration of $1 / 2$ hour do not currently exist. As a consequence, we provide a discussion that adjusts the cost for the shorter duration battery storage given the battery sizes currently available.

Typically, Na-S batteries can provide five times the rated power for up to 5 minutes (Kamibayashi et al. 2002). In our application, (as was discussed in Section 3.2.3.1), the peak power occurs for only short durations of 1-2 minutes. Hence, using the peak power (rather than the rated power) would still be conservative as a sizing criterion for the battery. If the peak power needed is $1 \mathrm{MW}$ and energy needed is $1 \mathrm{MWh}$, the battery size currently available and sufficient for the $1 \mathrm{MW} / 1 \mathrm{MWh}$ requirements would be 0.2 MW/1.36 MWh, because the energy/rated power (E/P) ratio is 6.8 for Na-S batteries. Hence, sizing of the battery is determined by the peak power needs.

While the sizing discussion above is appropriate for batteries currently available, we have assumed that as energy storage applications in utilities become more diverse, $\mathrm{Na}-\mathrm{S}$ will be available in various $\mathrm{E} / \mathrm{P}$ ratios, with the batteries being able to deliver peak power equal to twice the energy content (1 to 2MW/MWh) for 1-2 minutes. For Li-ion, the ability of batteries to provide 1 to $2 \mathrm{MW} / \mathrm{MWh}$ has been well demonstrated. Hence, the batteries were sized per the energy requirements.

\section{A.1.2 Capital Cost}

The cost of the PCS was estimated from the $\$ / \mathrm{kW}$ values available in the literature. It should be noted that the rated power of PCS is typically specified for continuous power. For durations of seconds to minutes, the peak power would be at least two times higher than the rated power for PCS, which would bring costs down.

An extensive search for capital costs of the Na-S battery system was conducted. In some publications, the battery energy storage system costs were given in terms of $\$ / \mathrm{kW}$, while they were given as $\$ / \mathrm{kWh}$ in others. For Na-S batteries, the long-term cost was $\sim 250 / \mathrm{kWh}$ (Schoenung and Hassenzahl 2003; Schoenung 2001; Gyuk and Eckroad 2003; Boyes; Kamibayashi et al. 2002), while the cost for PCS was $\$ 150$ to $\$ 260 / \mathrm{kW}$. The cost of the batteries was $\$ 1800$ to $\$ 2000 / \mathrm{kW}$ (Greenberg et al.; Kamibayashi et al. 2002), and $\$ 3080 / \mathrm{kW}$ (EAC 2008). The cost for the battery system including PCS and 
BOP was $\$ 2400$ to $\$ 2500 / \mathrm{kW}$ (Nourai 2007; Kishinevsky 2006). While these numbers vary widely, it should be noted that the long-term costs provided in $\$ / \mathrm{kWh}$ are only projections, and are not necessarily reflective of actual costs.

A detailed explanation of PCS and BOP costs was provided by Gyuk and Eckroad (2003). Type I PCS is maintained in hot standby and is operated continuously for durations greater than 30 seconds. Type II PCS may be employed for applications allowing 10 minutes advance notice, while Type III is used for durations less than 30 seconds. For this work, Type I describes the PCS most closely. The cost for Type I PCS as a function of output power P is given by Equation A.1:

$$
\frac{\$}{k W}=300 \cdot p^{-0.3}
$$

This provides a range of $\$ 40-\$ 300 / \mathrm{kW}$ for applications needing $1 \mathrm{GW}-1 \mathrm{MW}$ power. Clearly, as the system power increases, costs for PCS drops. Type I PCS costs are also given by Equation A.2:

$$
\frac{\$}{k W}=13500 \cdot V^{-0.59}
$$

where $\mathrm{V}$ is the minimum voltage on the battery side, and ranges from $300-3000 \mathrm{~V}$. Since the power needs in this study are in the order of a few GWs, for a minimum voltage of $3000 \mathrm{~V}$, the PCS cost would be $\$ 120 / \mathrm{kW}$. For this work, we have assumed a cost of $\$ 200 / \mathrm{kW}$, where the power corresponds to half the peak power, (or $\$ 100 / \mathrm{kW}$ based on peak power).

BOP costs include various components: systems integration (in $\$ / \mathrm{kW}$ ) not covered by PCS, costs for project engineering, grid connection (transformers), land, foundation, and buildings (Gyuk and Eckroad 2003). The BOP systems integration cost accounts for any underestimation of integration costs for PCS and energy storage, especially for pre-commercial systems. A value of $\$ 100 / \mathrm{kW}$ was proposed for precommercial systems, and $\$ 50 / \mathrm{kW}$ for commercial systems (Gyuk and Eckroad 2003). For this work, only systems integration costs in terms of $\$ / \mathrm{kW}$ are included with the cost being $\$ 100 / \mathrm{kW}$, based on power corresponding to half of peak power.

Table A.1 summarizes the literature review of current and future capital cost for Na-S battery systems. The value of $\$ 180-\$ 250 / \mathrm{kWh}$ provided by various sources is a long-term estimate. The $\$ 2500 / \mathrm{kW}$ value correspond to installed cost in the year 2006 for the Long Island Bus Project [9], while the $\$ 3000 / \mathrm{kW}$ provided by Nourai (Nourai 2010) was the actual installed cost for the year 2009 for a 7MW/48MWh battery system. The latter value was used for current price of a Na-S system. The cost of the battery portion was estimated by subtracting $\$ 200 / \mathrm{kW}$ for PCS and $\$ 100 / \mathrm{kW}$ for BOP, to yield $\$ 2700 / \mathrm{kW}$. Using a factor of 6-6.8 for $\mathrm{E} / \mathrm{P}_{\text {rated }}$ for Na-S batteries, the unit energy cost for the battery works out to $\$ 390-\$ 440 / \mathrm{kWh}$. Since the energy/power ratio varies for the different scenarios addressed in this report, the batteries have been costed using $\$ / \mathrm{kWh}$ numbers. For long-term cost, $\$ 250 / \mathrm{kWh}$ was used for the battery, along with $\$ 150 / \mathrm{kW}$ for PCS and $\$ 100 / \mathrm{kW}$ for BOP. 
Table A.1. Summary of Current Capital Cost Diversity for Na-S Systems

\begin{tabular}{|c|c|c|c|c|}
\hline $\begin{array}{l}\$ / \mathrm{kWh} \\
\text { current }\end{array}$ & $\begin{array}{l}\$ / \mathrm{kW} \\
\text { current }\end{array}$ & $\begin{array}{l}\$ / \mathrm{kWh} \\
\text { future }\end{array}$ & Notes & Source \\
\hline & & $180-250$ & Battery & $\begin{array}{l}\text { (Boyes; Kamibayashi et al. 2002; Schoenung and Hassenzahl } \\
\text { 2003; Gyuk and Eckroad 2003; Schoenung 2001) }\end{array}$ \\
\hline & & 818 & System & (Gyuk and Eckroad 2003) \\
\hline & 1800 & & Battery & (Greenberg et al.) \\
\hline & 3080 & & Battery & (EAC 2008) \\
\hline & $2400-2500$ & & System & ${\text { (Nourai } 2007^{(\mathrm{a})} \text {; Kishinevsky 2006 }}^{(\mathrm{b})}$ ) \\
\hline & 3000 & & System & (Nourai 2010) \\
\hline & $150-240$ & & PCS & $\begin{array}{l}\text { (Schoenung and Hassenzahl 2003; Schoenung 2001; } \\
\text { Kamibayashi et al. 2002; Boyes) }\end{array}$ \\
\hline & 100 & & BOP & (Kamibayashi et al. 2002) \\
\hline \multirow[t]{3}{*}{50} & & & BOP & (Boyes) \\
\hline & 100 & & $\mathrm{BOP}$ & (Gyuk and Eckroad 2003) \\
\hline & $150-450$ & & $\mathrm{PCS}^{(\mathrm{c})}$ & (Gyuk and Eckroad 2003) \\
\hline
\end{tabular}

(a) More reliable 2007 numbers.

(b) Reliable - 2006 Long Island Bus actual installation numbers.

(c) For short duration application, cost is low; for long duration, cost is higher.

The life cycles relationship with DOD of Na-S battery is depicted in Figure A.1. Therefore, in the battery sizing model, we put one variable called sizing factor to size up or size down the battery energy capacity, thus determining cycle life. It is also assumed that the calendar life of the batteries is 15 years.

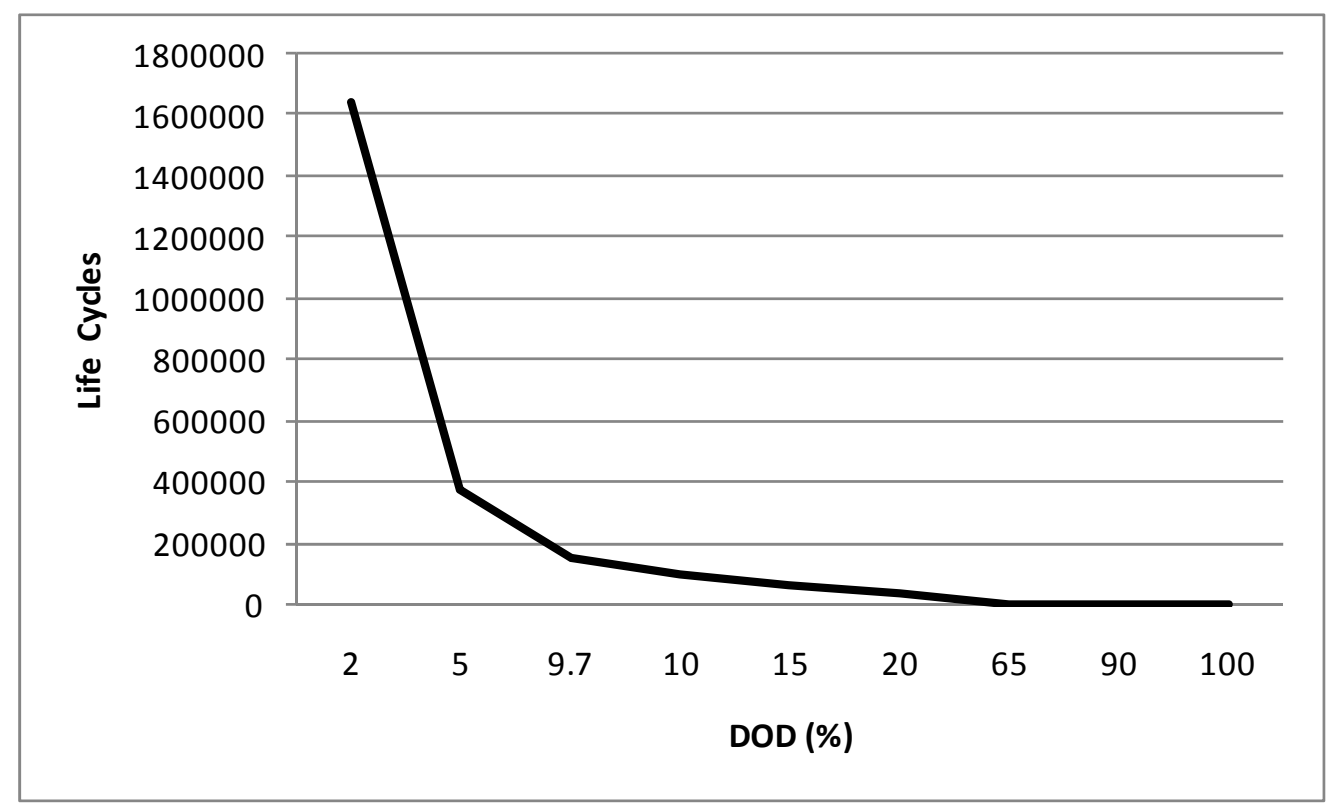

Figure A.1. Na-S Life Cycles versus DOD Curve 


\section{A.1.3 Fixed O\&M Cost}

The PCS consists of equipment necessary for energy transfer between the grid and energy storage system. The BOP is a catch-all for anything not covered by PCS. The O\&M costs for BOP have not been included in this analysis, since such costs are expected to be uniform across all technologies. In the next analysis, we plan to include uniform BOP costs, since it does affect the price tag for each technology. For Na-S batteries, fixed O\&M costs given in the literature varied over a wide range without any consistency on what services are included and which are excluded from the fixed O\&M cost. The low figures start at \$0.5/kW-year (Lamont 2004; Gyuk and Eckroad 2004) and go up to \$51/kW-year (Schoenung and Hassenzahl 2003; Gyuk and Eckroad 2003), which includes insurance and property taxes. For the purpose of this study, we used $\$ 3 / \mathrm{kW}$-year as the fixed O\&M cost.

\section{A.1.4 Variable O\&M Cost}

Variable costs have traditionally been reported in cents/kWh, and ranged from 0.4-0.7 cents/kWh, where $\mathrm{kWh}$ is the cumulative energy out of the battery (Lamont 2004; Schoenung et al. 1996). The variable O\&M cost for Na-S systems for various applications ranged from \$2.6-\$13.4/kW-year (Gyuk and Eckroad 2003). An average of these values yields $\$ 7 / \mathrm{kW}$-year, while conversion of this to $\$ / \mathrm{kWh}$ yields $0.4-0.8$ cents $/ \mathrm{kWh}$. We used 0.7 cents per $\mathrm{kWh}$ in this study. Table A.2 summarizes the O\&M cost estimates as found in the literature.

Table A.2. Summary of O\&M Fixed and Variable Costs for Na-S Battery

\begin{tabular}{lll}
\hline \multicolumn{1}{c}{ Fixed O\&M (\$/kW-year) } & \multicolumn{1}{c}{ Variable O\&M } & \multicolumn{1}{c}{ Reference } \\
\hline 3 & $16.9(\$ / \mathrm{kW}-$ year $)$ & (Gyuk and Eckroad 2004) \\
20 & & (Schoenung and Hassenzahl 2003) \\
$13-51(\$ 2 / \mathrm{kW}$-year for PCS) & $2.6-13.4(\$ / \mathrm{kW}$-year) & (Gyuk and Eckroad 2003) \\
0.5 & 0.7 cents $/ \mathrm{kWh}$ & (Lamont 2004) \\
1.5 & 0.5 cents $/ \mathrm{kWh}$ & (Schoenung et al. 1996) \\
3 & 0.7 cents $/ \mathrm{kWh}$ & Selected for study \\
\hline
\end{tabular}

\section{A.1.5 Efficiency}

For Na-S batteries, the AC-AC round trip efficiency was in the range of 0.75-0.85 (Kishinevsky 2006; Schoenung and Hassenzahl 2003; Schoenung 2001; Technology Insights 2005). We chose a roundtrip efficiency of 0.78 . For the Li-ion battery, the round trip efficiency was estimated to be 0.8 (Rastler et al. 2007). These figures correspond to battery system efficiencies that include all of the losses in the PCS. The specific PCS round trip efficiency was estimated to be 0.95 . The roundtrip efficiency is expected to change as a function of charge and discharge rate. For this analysis, the efficiency is kept constant for all rates. 


\section{A.2 Li-ion Battery}

\section{A.2.1 Battery Sizing}

Li-ion batteries from various applications have various power to energy (P/E) ratios, ranging from 60 for hybrid EVs (HEVs) to 4-16 for PHEV batteries (Rousseau 2007). In the transportation sector, $\mathrm{P}$ is defined as the power delivered by the battery for 2 seconds. For our application, the minimum resolution is 1 minute. Typically, Li-ion batteries can be discharged continuously at the maximum rate of $2 \mathrm{C}$ for $\sim 20$ minutes, with a P/E ratio of 1.3, where $\mathrm{C}$ is the energy capacity of the battery in Wh. For a 1-2 minute duration, it can be assumed that the $\mathrm{P} / \mathrm{E}$ for Li-ion batteries is $\sim 2$. This value will vary with the battery design, with high power battery having larger P/E ratios. Hence, in order to determine the actual cost of a battery, it must be determined whether power or energy is limiting. It is important to consider not just the maximum power requirements, but how long this maximum power will be needed continuously. This will fix the smallest battery energy content that would meet the power requirement after taking into account battery degradation.

\section{A.2.2 Capital Costs}

Present day Li-ion batteries cost ranges from \$1,015-\$1,450/kWh (Divya and Østergaard 2009) and $\$ 1000 / \mathrm{kWh}$ (Howell 2009). Other cost values given in $\$ / \mathrm{kW}$ are $\sim \$ 1070 / \mathrm{kW}$ (EAC 2008) and $\$ 970 / \mathrm{kW}$ (Gyuk and Eckroad 2003) with the higher cost including PCS and BOP costs. These numbers can be confusing, since the $\$ / \mathrm{kW}$ cost would equal $\$ / \mathrm{kWh}$ for a 1 -hour application, while it would be twice the $\$ / \mathrm{kWh}$ value for a 2-hour application. Telephone conversations with various battery manufacturers yielded a current price of $\$ 700$ to $\$ 1,500 / \mathrm{kWh}$, with the price for large volume sales of 1000 batteries or more in 5 years estimated at $\$ 500-\$ 700 / \mathrm{kWh}$, where the cost includes battery management + mark-up (Riedel 2010). Cost estimation models from Argonne National Laboratory (ANL), Tiaxx and The US Advanced Battery Consortium (USABC) (Nelson et al. 2009; Barnett et al. 2009; USCAR 2007), when normalized to the same total energy, provided a cost of $\$ 440, \$ 415$, and $\$ 450 / \mathrm{kWh}$, respectively, for large volume production, or an average of $\$ 420 / \mathrm{kWh}$. The cost to be used for this study was assumed to be $\$ 510 / \mathrm{kWh}$ long-term for high volume production using the average of $\$ 600 / \mathrm{kWh}$ and $\$ 420 / \mathrm{kWh}$. The long-term costs were $\$ 510 / \mathrm{kWh} * 2.35=\$ 1200 / \mathrm{kW}$. Table A.3 summarizes the cost information for Li-ion batteries. 
Table A.3. Summary of Current Capital Cost Diversity for Li-Ion Systems

\begin{tabular}{|c|c|c|c|}
\hline$\$ /$ kWh current & $\$ / \mathrm{kW}$ current & $\begin{array}{c}\text { \$/kWh } \\
\text { high volume }\end{array}$ & Source \\
\hline 700-1000 Euros & -- & -- & (Divya and Østergaard 2009) \\
\hline \multirow[t]{3}{*}{1000} & -- & & (Howell 2009) \\
\hline & $\begin{array}{l}1070 \text { includes PCS } \\
\text { and BOP }\end{array}$ & & (EAC 2008) \\
\hline & 970 & & (Gyuk and Eckroad 2003) \\
\hline $\begin{array}{c}1500 \text { (60,000 } \\
\text { cells/year) }\end{array}$ & & $\begin{array}{c}1000-1250 \\
(180,000 \text { cells/year })\end{array}$ & $\begin{array}{l}\text { Lithium Technology Corp. } \\
\text { (LTC) (Hazel 2010) }\end{array}$ \\
\hline 700 & & & $\begin{array}{l}\text { Lithium Technology Corp. } \\
\text { (LTC) (Hazel 2010) }\end{array}$ \\
\hline $\begin{array}{c}1000-1200(1000+ \\
\text { batteries/year) }\end{array}$ & & $\begin{array}{l}\text { 500-700 ( } 5 \text { years } \\
\text { from now) }\end{array}$ & Compact Power (Riedel 2010) \\
\hline \multirow[t]{4}{*}{1000} & & & BASF (Chintawar 2010) \\
\hline & & 415 & ANL (Nelson et al. 2009) \\
\hline & & $440^{(a)}$ & TIAX (Barnett et al. 2009) \\
\hline & & 450 & USABC (USCAR 2007) \\
\hline
\end{tabular}

The life cycles versus DOD curve of Li-ion batteries are shown in Figure A.2.

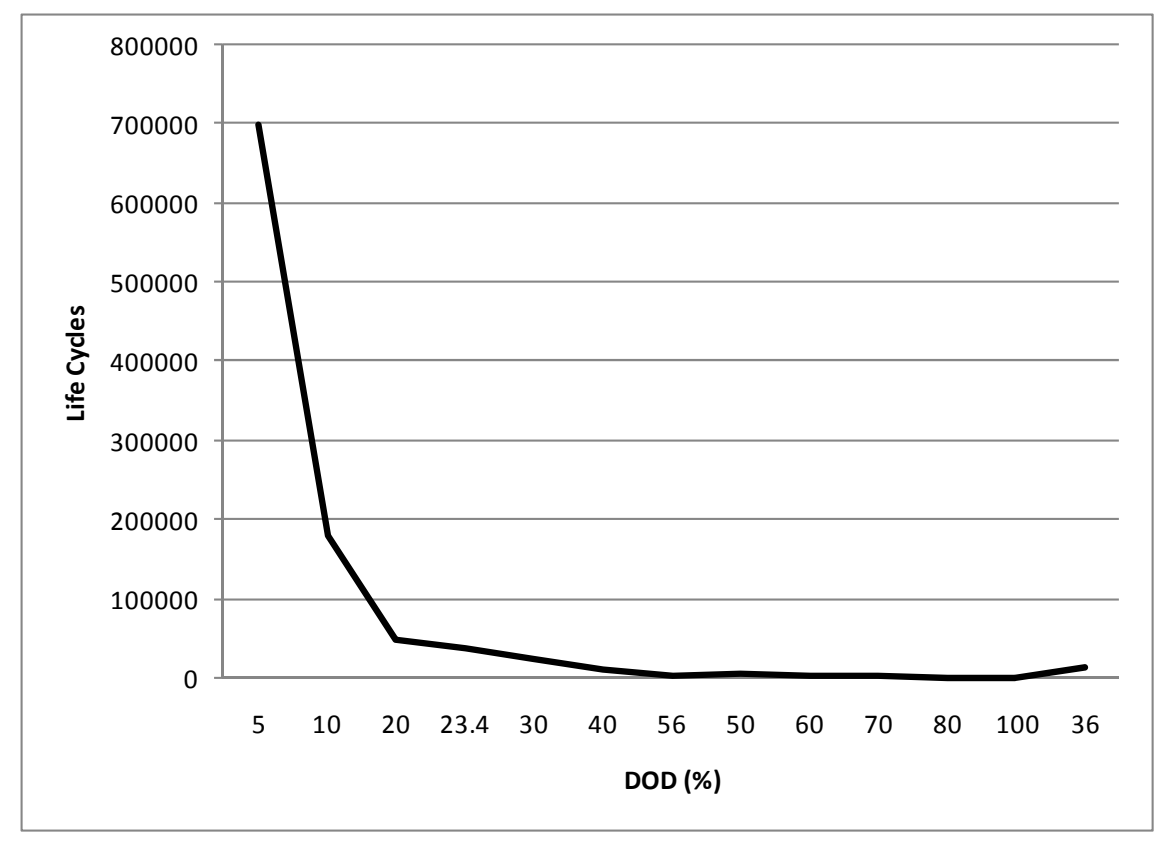

Figure A.2. Li-Ion Battery Life-Cycle versus DOD Curve 


\section{A.3 Pumped Hydroelectric (PH) Systems}

\section{A.3.1 Capital and O\&M Costs}

For PH systems, the capital cost is provided in $\$ / \mathrm{kW}$. Most systems provide this information by including PCS and BOP costs. For this analysis, BOP costs for PH will be neglected, since the values provided in the literature are as small as $\$ 4 / \mathrm{kWh}$. Additional capital cost information was provided by Rick Miller of Renewable Energy Services in several e-mail communications (Miller 2010). The capital costs for single speed $\mathrm{PH}$ systems are in the range of $\$ 1,500$ to $\$ 2,500 / \mathrm{kW}$, while the range for variable speed pumps is $\$ 1,800$ to $\$ 3,200 / \mathrm{kW}$. This cost is broken down into three parts:

1. Pump/turbine and motor/generator costs, $(\$ 600 / \mathrm{kW}$ for single speed and $\$ 850 / \mathrm{kW}$ for variable speed units)

2. Hydro-mechanical equipment, transformers, switchgears, remaining BOP

3. Engineering/design services, civil construction, excavations and construction for water conveyance system, upper and lower dams and reservoirs.

The pump and turbine costs typically for pumped hydro projects vary significantly, and have been reported to be as low as $\$ 78 / \mathrm{kW}$ to $\$ 264 / \mathrm{kW}$ (GE Energy 2004; Alstom 2009). Since these costs are about $33 \%$ of total system cost, they also contribute to large variation in system cost, in addition to the siting-related contribution.

A range of values obtained from the literature on capital costs, O\&M fixed and O\&M variable for PH systems is shown in Table A.4. While replacement costs are minimal, the generators need rewinding every 20-25 years.

The round trip efficiency is $80 \%$ to $82 \%$, and does not include transmission losses. It should be noted that the reported $75 \%$ efficiency probably includes transmission losses. 
Table A.4. Capital Costs for Pumped Hydro Systems

\begin{tabular}{|c|c|c|c|c|}
\hline$\$ / \mathrm{kW}$ & $\begin{array}{l}\text { O\&M Fixed } \\
\$ / \mathrm{kW} \text {-year }\end{array}$ & $\begin{array}{l}\text { O\&M Variable } \\
\text { cents/kWh }\end{array}$ & $\begin{array}{l}\text { Efficiency } \\
\text { AC-AC }\end{array}$ & Reference \\
\hline 1000 & 2.5 & Very small & 0.75 & (Schoenung and Hassenzahl 2003) \\
\hline 600 & 3.8 & 0.38 & 0.87 & (Schoenung 2001) \\
\hline 1483 & & & & (Geadah 2009) \\
\hline 1552 & & & & $\left(_{(G e a d a h ~ 2009)}\right)^{(a)}$ \\
\hline \multirow[t]{3}{*}{$>350 € / \mathrm{kW}$} & & & $0.70-0.80$ & (Fodstad) \\
\hline & & 2 & 0.75 & (Rahman 1990) \\
\hline & & & 0.75 & (First Hydro Company 2009; Jenkinson 2005) \\
\hline 1500 & & & 0.82 & $(\mathrm{NHC} 2007)^{(\mathrm{c})}$ \\
\hline 1000 & & & & (Boyes) \\
\hline $1800-3200^{(\mathrm{d})}$ & & & $0.78-0.82$ & (Miller 2010) \\
\hline $517,583^{(\mathrm{e})}$ & 4.6 & & $0.80^{(\mathrm{f})}$ & (Figueiredo et al. 2006) \\
\hline $\begin{array}{l}1100-2000^{(\mathrm{g})} \\
1700^{(\mathrm{i})}\end{array}$ & 4.3 & $0.43^{(\mathrm{h})}$ & $0.60-0.78$ & $\begin{array}{l}\text { (Lipman et al. 2005) } \\
\text { (Miller 2010) }\end{array}$ \\
\hline \\
\hline \multicolumn{5}{|c|}{$\begin{array}{l}\text { (a) } 2016-2020 \text { costs. } \\
\text { (b) At Dinorwig ( } 1800 \mathrm{MW} 1.5 \text { min start-up). }\end{array}$} \\
\hline \multicolumn{5}{|c|}{500 MW fully dispatchable in 15 seconds with unit spinning, 10 min black start. } \\
\hline \multicolumn{5}{|c|}{ d) $\$ 1500-\$ 2500 / \mathrm{kW}$ for single speed. } \\
\hline \multicolumn{5}{|c|}{ \$517/kW Grand Cache 218 MW, \$583/kW Kneehills 194 MW. } \\
\hline \multicolumn{5}{|c|}{ Efficiency set at 0.8 , not measured. } \\
\hline \multicolumn{5}{|c|}{ Projected cost for year 2020 was $\$ 800 / \mathrm{kW}$. } \\
\hline \multicolumn{5}{|c|}{ Referenced Schoenung et al. (1996). } \\
\hline \multicolumn{5}{|c|}{ \$245/kW for pump turbine, motor generator and power electronics. } \\
\hline
\end{tabular}

\section{A.3.2 Duration Between Various Modes}

The response time for PH systems is fast, achieving high ramp rates as high as $3 \mathrm{MW} / \mathrm{s}$ (First Hydro Company 2009). The calendar life was estimated to be 50 years (Schoenung 2001). Figure A.3 shows typical start and stop times for PH systems. It takes about 12 seconds for the system to go from a spinning to generation state, while the corresponding time for transition to pumping is 30 seconds. The average time from shutdown to generation is 90 seconds, while shutting down the system from the pumping state takes about 6 minutes.

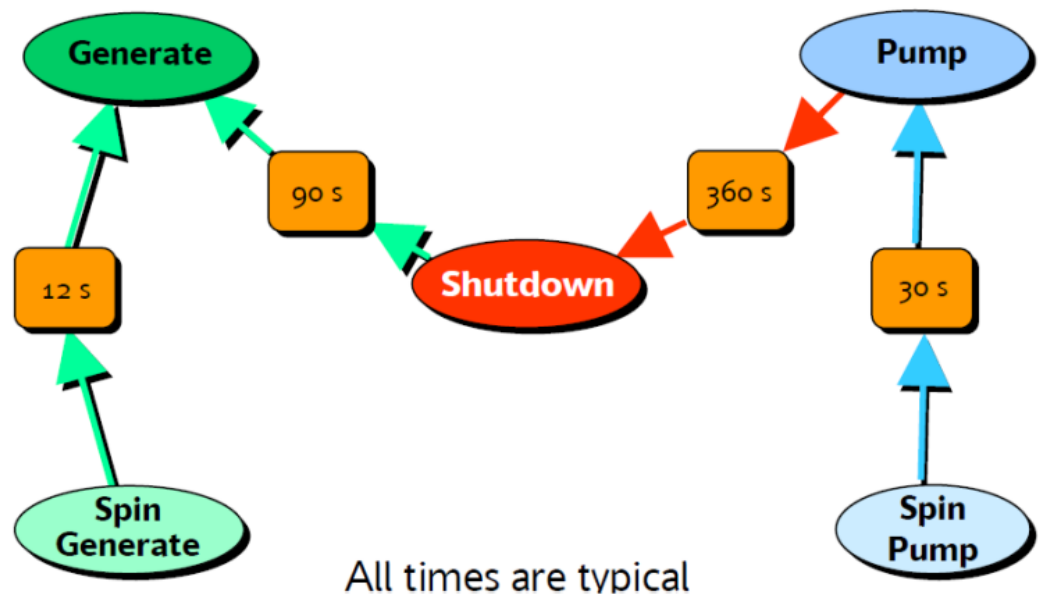

Figure A.3. Typical Duration Between Various Modes at the Dinorwig PH System (Jenkinson 2005) 
After several consultations with turbine and pumped hydro storage system experts, we assume a delay of 4 minutes to switch operating modes in each direction (pumping to generation and vice versa).

\section{A.4 Compressed Air Energy Storage}

\section{A.4.1 Current Status}

There are two operating CAES plants in the world, a 300 MW/3h plant in Huntorf, Germany, commissioned in 1978 and a 110 MW/26h plant located in McIntosh, Alabama, which was commissioned in 1991. The Huntorf plant, designed by ABB has a rated output of $290 \mathrm{MW}$ over 3 hours (Succar and Williams 2008), with an overall efficiency of $42 \%$ and a heat rate of $5870 \mathrm{~kJ} / \mathrm{kWh}$ lower heating value LHV. The McIntosh plant, designed by Dresser-Rand, recuperates the turbine exhaust heat, thus improving the overall efficiency to 53\%, with a heat rate of $4330 \mathrm{~kJ} / \mathrm{kWh}$ LHV (Succar and Williams 2008; BINE 2007). Dresser-Rand supplied all the rotating equipment for this plant, while Energy Storage and Power (ESPC) was responsible for engineering, design, manufacture, testing and commissioning of the plant (Lucas and Miller 2010; Nakhamkin et al. 2010). Other proposed projects such as the limestone mine in Norton Ohio (800 MW) and plans by Ridge Energy Storage \& Grid Services L.P. for several CAES installations in Texas have been described by Succar and Williams (2008). These plants are termed first generation, with compressed air being heated by a high pressure and low pressure combustor, and the heated air passing through a high and low pressure expander. In the second generation CAES plant developed by ESPC, one-third of the power is generated by a combustion turbine which heats atmospheric pressure air. The exhaust from the CT is used to heat the stored compressed air, which is passed through an expander to generate power. Separate power trains are used for the compression and expansion sections in order to improve reliability at the expense of higher cost (Nakhamkin et al. 2010).

\section{A.4.2 CAES Performance and Life}

The energy and power components for CAES are separate. The CAES has a start-up time of $<5$ minutes during compression to full load, while the corresponding start-up time in the power generation mode is $<10$ minutes (Miller 2011). This affects the sizing of the energy storage system, since a battery needs to be used during the changeover period. Additionally, if the CAES is used as a load at night and as a generator during the day, it needs to be oversized.

The cycle life, while not known, is expected to be $>10,000$ cycles based on the 20-30 year life at the Huntorf and McIntosh plants and the 5000+ combined starts for generation and compression at the McIntosh plant (Hoffman 2008). A wide range of round trip efficiency (48\%-90\%) for CAES has been quoted in the literature using different definitions (Drury 2011; Macchi and Lozza 1987; Miller 2011; Succar and Williams 2008). The efficiency is in the 77\%-89\% range if it is defined as the ratio of electricity generated to the sum of the electricity input to the compressor and the electricity that could have been generated by the natural gas in a combustion turbine; while it is approximately $66 \%$ for a ratio of output electricity adjusted by subtracting the electricity that could have been generated by the natural gas in a combustion turbine to the electricity input to the compressor (Succar and Williams 2008). In this work, efficiency is defined as the ratio of electricity generated to total energy input to the system, and is $50 \%$. It is anticipated that efficiency as high as 0.7 can be obtained for adiabatic/isothermal CAES (Drury et al. 2011), but the technology is not yet mature. A summary of novel concepts for the next generation CAES plants was described by Nakhamkin et al. (2009). 
The ramp rates for CAES were reported in the range of $17 \%-40 \%$ rated power/minute (Gyuk and Eckroad 2003; Ridge 2005; Succar and Williams 2008; Nakhamkin et al. 2010; Miller 2011). A ramp rate of $30 \%$ of rated power per minute has been assumed in this work.

\section{A.4.3 CAES Capital and O\&M Cost}

For CAES systems, the capital costs are provided in $\$ / \mathrm{kW}$. For most systems, the BOP and PCS costs are also included. Some of the main suppliers of CT are GE, Siemens and Westinghouse, while compressors are supplied by MAN Turbo, Dresser-Rand, Mitsubishi Hitachi, Rolls-Royce and Ingersoll Rand (Nakhamkin 2008). The cost for the current generation 110 MW CAES plant was estimated to be $\$ 1250 / \mathrm{kW}$, with second generation systems estimated to be $\$ 750 / \mathrm{kW}$, while systems based on advanced concepts were estimated to be in the $\$ 500-\$ 560 / \mathrm{kW}$ range (Daniel 2008; Nakhamkin et al. 2010). The capital cost was quoted at $\$ 1200 / \mathrm{kW}$ for the recently cancelled Iowa project (Cavallo 2011).

Table A.5 summarizes the CAES capital costs from the literature. The power-related capital costs for CAES is in the range $\$ 500-\$ 1750 / \mathrm{kW}$, while the energy related costs are approximately $\$ 3 / \mathrm{kWh}$ for salt dome storage. The energy related costs varies with storage type, with porous rock storage and surface storage costs higher by more than an order of magnitude. For this work, the capital cost was assumed to be $\$ 1000 / \mathrm{kW}$ in 2011 and $\$ 850 / \mathrm{kW}$ in 2020 , taking into account an increase in motor/generator material cost that is expected to counter some of the advances anticipated, with energy related costs assumed to be $\$ 3 / \mathrm{kWh}$.

Table A.5. Summary of Capital Cost Diversity for CAES Systems

\begin{tabular}{|c|c|c|c|c|c|}
\hline $\begin{array}{l}\text { Capital Cost } \\
(\$ / \mathrm{kW})\end{array}$ & $\begin{array}{c}\text { Capital Cost } \\
(\$ / \mathrm{kWh})\end{array}$ & $\begin{array}{l}\text { O\&M Fixed } \\
\text { (\$/kW-year) }\end{array}$ & $\begin{array}{c}\text { O\&M } \\
\text { Variable } \\
\text { (cents/kWh) }\end{array}$ & $\begin{array}{l}\text { Ramp Rate \% } \\
\text { Rated } \\
\text { Power/min }\end{array}$ & Reference \\
\hline 560 & 3 & 1.2 & 0.15 & & (Cavallo 1995) \\
\hline 425 & 3 & 2.5 & & & $\begin{array}{l}\text { (Schoenung and } \\
\text { Hassenzahl 2003) }\end{array}$ \\
\hline $440^{(\mathrm{a})}$ & 1 & 13 & 0.2 & $10 \%-25 \%{ }^{(\mathrm{b})}$ & (Gyuk 2003) \\
\hline 430 & $40^{(\mathrm{c})}$ & $19-24.6$ & & $10 \%-25 \%{ }^{(\mathrm{b})}$ & (Gyuk 2003) \\
\hline $500-850$ & & & 0.3 & & $\begin{array}{l}\text { (van der Linden 2006, } \\
\text { Miller 2011) }\end{array}$ \\
\hline 350 & $1 / 0.1 / 30 / 30^{(\mathrm{d})}$ & 6 & & & (Herman 2003) \\
\hline 350 & $1.75 / 40^{(\mathrm{e})}$ & & & & (Eckroad 2004) \\
\hline $\begin{array}{l}1700 \text { (72 MW } \\
\text { adiabatic CAES) }\end{array}$ & & 6 & & & (Nakhamkin et al. 2007) \\
\hline $800-850$ & & & & $\begin{array}{l}27 \% \\
18 \%\end{array}$ & $\begin{array}{l}\text { (Nakhamkin et al. 2009) } \\
\text { (Ridge Energy 2005) }\end{array}$ \\
\hline $\begin{array}{l}890 \\
750-800\end{array}$ & & $<5^{(\mathrm{f})}$ & & & (Greenblatt 2005) \\
\hline 580 & 1.7 & & & $7 \%-14 \%$ & $\begin{array}{l}\text { (Succar and Williams } \\
\text { 2008) }\end{array}$ \\
\hline $960-1250$ & $60-120$ & & & & $\begin{array}{l}\text { (Rastler 2010, Daniel } \\
\text { 2008) }\end{array}$ \\
\hline $850-900^{(\mathrm{g})}$ & $85-90$ & & & $12 \%-27 \%{ }^{(\mathrm{h})}$ & (Nakhamkin et al. 2010) \\
\hline 500 & & & 0.3 & $\begin{array}{l}20 \%-35 \% \\
40 \% \\
10 \%-20 \%{ }^{(i)}\end{array}$ & $\begin{array}{l}\text { (Miller 2011) } \\
\text { (Schainker et al. 2010) } \\
\text { (Lucas 2010) }\end{array}$ \\
\hline
\end{tabular}


(b) $10 \% /$ min generation, $25 \% /$ min compression.

(c) $10 \mathrm{MW}$ AC surface storage.

(d) Salt/porous/hard rock/surface.

(e) 1.75 for salt mine, 40 for surface.

(f) Replacement cost $<\$ 5 / \mathrm{kW}$-year.

(g) $\mathrm{S}$ gen 2 plants, included energy costs for $10 \mathrm{~h}$ storage. $\$ / \mathrm{kWh}$ obtained by dividing $\$ / \mathrm{kW}$ by 10 .

(h) Load following in the $20 \%-100 \%$ of capacity within 3-5 minutes.

(i) $10 \% / \mathrm{min}$ generation, $20 \% / \mathrm{min}$ compression.

\section{A.5 Flywheel Energy Storage}

\section{A.5.1 Current Status}

The major manufacturers of flywheels (Gyuk 2003) include Beacon Power, Tribology Systems Inc, Velkess Flywheels and Amber Kinetics, with about half the manufacturers using steel rotors, which is appropriate for low energy power quality applications. Among the current manufacturers, Beacon Power has developed flywheels that can provide $25 \mathrm{kWh}$ at a power of $100 \mathrm{~kW}$, and is currently developing $100 \mathrm{~kW} / 100 \mathrm{kWh}$ modules (Lazarewicz 2011). Flywheel systems using such modular flywheels have been developed to provide $20 \mathrm{MW}$ power and an energy of $5 \mathrm{MWh}$, with a goal of $100 \mathrm{MWh}$. In terms of losses, based on testing of the $100 \mathrm{~kW}$ modules on CAISO and NYISO grids, the total losses were $7.09 \%$ per year, with $7 \%$ corresponding to efficiency losses, and $0.09 \%$ to standby losses. For their $100 \mathrm{~kW}$ $25 \mathrm{kWh}$ module, all of the energy is usable, since it is oversized to $40 \mathrm{kWh}$. The flywheel uses a permanent magnet high speed motor. For this module, the system roundtrip efficiency was 85\%, with 13\% losses attributed to the PCS (Lazarewicz 2011). $40 \mathrm{kWh}$ systems running at $25 \mathrm{~kW}$ peak power and $4 \mathrm{~kW}$ continuous power developed by Tribology Systems Inc. have ceramic bearings, with an estimated energy loss of $<0.03 \%$ per hour, allowing the flywheels to operate unpowered for over 4 months (Sibley 2011a, 2011b). Velkess has developed $10 \mathrm{~kW} / 80 \mathrm{kWh}$ systems targeting telecommunications (Gray 2009). Amber Kinetics has a demonstration scheduled in 2013 with California ISO, with initial development of $20 \mathrm{~kW}, 5 \mathrm{kWh}$ system in Phase 1, followed by a target of $500 \mathrm{~kW}, 125 \mathrm{kWh}$ commercialscale prototype system, and a grid-connected demonstration system at the MWh level (Chiao 2011). Active Power and VYCON Energy are targeting UPS markets. Active Power has deployed over 2000 flywheels for UPS with a diesel generator to deliver 15-seconds ride through at peak power and 30 seconds at $50 \%$ of peak power (Active Power 2011). While the cost per $\mathrm{kW}$ was stated to be $\$ 330 / \mathrm{kW}$, this low cost could possibly be due to the small duration for which these systems are designed. As of now, Active Power is not participating in the regulation market. VYCON Energy has developed $300 \mathrm{~kW}$, $1.1 \mathrm{kWh}$ flywheel systems targeting the UPS market (Ulibas 2011). Other markets currently being targeted are capturing regenerative power from cranes, electric rail, and buses. The UPS market has low cycle life requirements, while in cranes, the flywheels cycle every 2 minutes for 8 hours. For this application, the flywheels are de-rated both in terms of power and energy. Their systems have standby losses of $2 \mathrm{~kW}$, and the efficiency during continuous operation is anticipated to be $97 \%$.

\section{A.5.2 Flywheels Performance and Life}

Flywheels are expected to last 25 years, with a cycle life of 125,000 at 100\% DOD (Lazarewicz 2011; Sibley 2011a; Gray 2011; Chiao 2011). While DOD typically determined cycle life for batteries, for flywheels, the wear and tear mainly depends on the rotational speed of the rotors. Hence, the number of 
charges to $100 \%$ state of charge (SOC) is expected to be more degradative compared to number of charges to $50 \%$ SOC. For example, the cycle life at $25 \%$ DOD was estimated to be 450,000 by Beacon Power.

As discussed earlier, the efficiency of the Beacon flywheels system is $98 \%$, with additional $13 \%$ losses from the PCS. The Tribology Systems Inc. (TSI) Flywheel has efficiency in the 95\%-97\% range due to low losses associated with the ceramic bearings. The roundtrip efficiency of the Velkess Flywheels kW sized Systems (Gray 2009) and the Amber Kinetics systems (Chiao 2011) is 85\%, while the efficiency for MW sized systems was estimated to be $90 \%-95 \%$ (Gray 2011). The ramp rate for the Velkess Flywheels is twice the rated power in less than a second using $250 \mathrm{kWh}$ modules (Gray 2011). Most flywheels are oversized since operating at 100\% DOD is not practical due to low efficiencies at low speeds. One unique feature of the TSI system is that over-sizing is not necessary, since the flywheel can be discharged down to low speeds without loss of efficiency (Sibley 2011c).

\section{A.5.3 Flywheels Capital and O\&M Costs}

Beacon Power signed a \$2 million contract with the New York State Energy Research \& Development Authority (NYSERDA) for partial funding of its $20 \mathrm{MW} / 5 \mathrm{MWh}$ frequency regulation plant in Stephentown, New York (Beacon 2010). The plant is expected to consist of 200 Smart Energy 25 flywheels (100 kW, $25 \mathrm{kWh}$ ), with a total estimated cost was \$25 million, out of which \$5 million was estimated to be installation cost (Lazarewicz 2011). This corresponds to $\$ 1,000 / \mathrm{kW}$ for the system excluding installation costs. The flywheel is a turnkey system, with a dc-dc converter stepping the voltage to $480 \mathrm{~V}$ dc, followed by a bi-directional inverter and a transformer for conversion to $115 \mathrm{kV}$ ac. This was a significant increase from the estimated \$10-\$12 million reported earlier (Rounds and Peek 2008). The estimated cost for a $250 \mathrm{kWh}$ TSI flywheels system was $\$ 200 / \mathrm{kWh}$, and was $\$ 165 / \mathrm{kWh}$ for a $1 \mathrm{MWH}$ system, with the cost inclusive of the motor/generator cost for charging and discharging the flywheel. These estimates were based on current carbon fiber prices, and are mainly sensitive to the energy content of the system due to the $>1$-h charge discharge periods (Sibley 2011c). The Velkess Flywheels have a flexible rotor and a passive magnet, with lower associated construction cost. For MW/MWh sized system, the costs were estimated to be $\$ 200 / \mathrm{kW}$ and $\$ 100 / \mathrm{kWh}$ (Gray 2011). Table A.6 shows a list of these costs. The capital cost in terms of $\$ / \mathrm{kWh}$ for steel, graphite and carbon fiber rotors are also listed. Amber Kinetics uses commercially off the shelf bearings and low cost high strength steel rotors, with a goal to reduce the unit energy cost for the rotors by a factor of 15 (Chiao 2011).

The O\&M costs are in the $\$ 20$-\$30/kW-year range (Walwalkar et al. 2006), \$18/kW-year (Gyuk and Eckroad 2003) and $2 \%$ of capital costs per year or $\$ 7 / \mathrm{kW}$-year for high power low energy systems (Taylor 1999), while O\&M variable cost was 0.1 cents/kWh (Gyuk and Eckroad 2003). For this study, fixed O\&M costs of $\$ 18 / \mathrm{kW}$-year and variable O\&M costs of 0.1 cents $/ \mathrm{kWh}$ were used. 
Table A.6. Summary of Capital Cost Diversity for Flywheel systems

\begin{tabular}{|c|c|c|c|c|c|}
\hline $\begin{array}{c}\text { Capital Cost } \\
(\$ / \mathrm{kW})\end{array}$ & $\begin{array}{l}\text { Capital Cost } \\
(\$ / \mathrm{kWh})\end{array}$ & $\begin{array}{l}\text { O\&M Fixed } \\
(\$ / \mathrm{kW} \text {-year) }\end{array}$ & $\begin{array}{c}\text { O\&M } \\
\text { Variable } \\
\text { (cents/kWh) }\end{array}$ & Efficiency (\%) & Reference \\
\hline $200-500(5 s)$ & & $\begin{array}{c}2 \% \text { of } \\
\text { capital costs }\end{array}$ & & & (Taylor et al. 1999) \\
\hline 800 (UPS) & & & & & (Taylor et al. 1999) \\
\hline 200-500 (few min) & & & & & $\begin{array}{l}\text { (Prodromidis and } \\
\text { Coutelieris 2012) }\end{array}$ \\
\hline $1000-3000(1 \mathrm{~h})$ & & & & & $\begin{array}{l}\text { (Prodromidis and } \\
\text { Coutelieris 2012) }\end{array}$ \\
\hline $\begin{array}{l}1000 \text { (Gen } 44 \\
\text { Beacon) }^{(\mathrm{a})}\end{array}$ & & & & $85 \%$ & (Lazarewicz 2011) \\
\hline & & 18 & 0.1 & & (Gyuk 2003) \\
\hline \multirow[t]{5}{*}{1630} & & & & & (Rounds and Peek 2008) \\
\hline & & & & & $\begin{array}{l}\text { (Liu and Jiang 2007, } \\
\text { Bolund 2007) }\end{array}$ \\
\hline & $38^{(\mathrm{c})}$ & & & & (Liu and Jiang 2007) \\
\hline & $104-290^{(\mathrm{d})}$ & & & & (Bolund 2007) \\
\hline & $165-250^{(\mathrm{e})}$ & & & $95 \%-97 \%{ }^{(f)}$ & (Sibley 2011b,c) \\
\hline $200^{(\mathrm{g})}$ & 100 & & & $90 \%-95 \%{ }^{(\mathrm{h})}$ & (Gray 2011) \\
\hline \multirow[t]{2}{*}{$\begin{array}{l}650(1 \mathrm{MW} / \\
0.25 \mathrm{MWh})\end{array}$} & & $20-30$ & & & (Walwalkar et al. 2006) \\
\hline & & & & $85 \%$ & (Chiao 2011) \\
\hline $\begin{array}{l}\text { (a) } 200 \mathrm{MW} \text { AC, s } \\
\text { (b) } 10 \% / \text { min gener } \\
\text { (c) } 10 \mathrm{MW} \text { AC sur } \\
\text { (d) Salt/porous/har } \\
\text { (e) } 1.75 \text { for salt mi } \\
\text { (f) Replacement c } \\
\text { (g) } \mathrm{S} \text { gen } 2 \text { plants, } \\
\text { (h) Load following } \\
\text { (i) } 10 \% / \text { min gener }\end{array}$ & $\begin{array}{l}\text { lt mine storage, } \\
\text { tion, } 25 \% / \text { min } \\
\text { ace storage. } \\
\text { I rock/surface. } \\
\text { e, } 40 \text { for surface } \\
\text { st }<\$ 5 / \mathrm{kW} \text {-year. } \\
\text { ncluded energy } \\
\text { in the } 20 \%-100 \\
\text { tion, } 20 \% / \mathrm{min} \text { c }\end{array}$ & $\begin{array}{l}\text { includes BOP } \\
\text { ompression. } \\
\text { costs for 10h st } \\
\text { o of capacity h } \\
\text { ompression. } \\
\end{array}$ & $\begin{array}{l}\text { f \$170/kW. } \\
\text { orage. \$/kWh } \\
\text { thin 3-5 min }\end{array}$ & ined by dividi & \$/kW by 10. \\
\hline
\end{tabular}

\section{A.6 Vanadium Redox Flow Batteries}

\section{A.6.1 Current Status}

Vanadium redox flow battery systems range from several small 5-kW units deployed in field trials to much larger installations. For example, a 15-kW/120-kWh system was deployed by Risø-DTU, Denmark, a 50 kW/200 kWh was installed by Kashima-Kita Electric Power in 1995, a 200 kW/800 kWh system was installed by VRB at the King Island wind farm in Australia, a $250 \mathrm{~kW} / 2 \mathrm{MWh}$ system built by VRB Power Systems was installed by PacificCorp in Castle Valley, Utah, and the 4MW/6MWh system built by Sumitomo Industries was installed at the 32MW Tomamae wind farm in northern Japan (Rastler 2010; Eckroad 2007; Yang et al. 2011; Steeley 2005; Zhang 2009; Skyllas-Kazacos 2010).

While most vanadium redox flow batteries operate in the $0^{\circ} \mathrm{C}-40^{\circ} \mathrm{C}$ temperature range, a wider $-10^{\circ} \mathrm{C}$ to $50^{\circ} \mathrm{C}$ range has been demonstrated ( $\mathrm{Li}$ et al. 2011). Widening the temperature range would lower costs associated with heat exchangers, while also increasing the life of the electrolyte. Efforts are also ongoing 
to reduce stack costs by developing higher performance stacks, lower-cost membranes, electrodes and bipolar plates. On the energy side, higher concentration electrolytes are being developed to increase energy density. System design issues being addressed include minimization of losses associated with pumping and shunt current.

\section{A.6.2 Performance and Life}

The efficiency of the system is mainly a function of the power density, and can be controlled to be in the $75 \%-85 \%$ range by varying the stack area for a desired power output. Hence, this provides an additional lever by designing the stacks appropriately for short duration applications such as balancing and regulation versus energy intensive applications such as arbitrage and load leveling. The cycle life of redox flow batteries depends on its use profile. Tokuda et al. (2000) targeted at least 1500 cycles over 10 years. In principle, the battery can be cycled an order of magnitude higher, as long as the charge voltage is maintained below the gas evolution range (Vanadiumsite 2011; Energystoragenews 2010; Vfuel 2011; Staudt). The Tomomae wind energy storage demonstration using a $4 \mathrm{MW} / 6 \mathrm{MWh}$ Sumitomo Electric Industries system has undergone $>200,000$ cycles after 3 years, thus indicating the life for VRB systems is mainly limited by calendar life rather than cycle life (Skyllas-Kazacos 2011).

\section{A.6.3 Vanadium Redox Flow Battery Capital and O\&M Costs}

A wide range of costs has been reported for redox flow batteries, with unit costs varying based on the power to energy ratio (Eckroad 2007; Gyuk 2003; Corey 2002). A comprehensive review of the vanadium redox flow battery systems deployment and cost analysis was recently published (Kear et al. 2011).

We have estimated the power and energy cost components in this work. The stack cost is mainly governed by separator costs, which currently are in the $\$ 500-\$ 800 / \mathrm{m}^{2}$ range. It is expected that in the next 10 years, the separator costs would drop to $\$ 200 / \mathrm{m}^{2}$ (Kannurpatti 2011). Felt electrode development with various forms of heat and chemical treatment was expected to enhance performance (Yang et al. 2011). The energy costs are mainly dependent on $\mathrm{V}_{2} \mathrm{O}_{5}$ costs, which peaked in 2005 at $\$ 27 / \mathrm{lb}$, and have stabilized at $\$ 10 / \mathrm{lb}$ (Eckroad 2007). With recycling, $\mathrm{V}_{2} \mathrm{O}_{5}$ costs are expected to contribute less in the future. The electrolyte is expected to be very stable, thus enabling reuse in the future. For this work, a 2020 cost estimation was prepared, assuming an anticipated drop in component costs and a 20\% anticipated increase in power density, which would decrease stack costs for a fixed power output. In addition, for both 2011 and 2020 costs, the unit power costs were divided by 1.4, which is the ratio of peak to rated power (Bindner 2010). In 2020, the capital cost used in this study was $\$ 131 / \mathrm{kWh}$ for the energy related component and $\$ 775 / \mathrm{kW}$ for the power-related component. Of the total power-related costs, $\$ 496$ are associated with stack costs and the economic life of the stack is assumed to be 10 years compared with 25 years on all other components. In 2011, power-related capital costs used were $\$ 1,111 / \mathrm{kW}$ for the entire system and $\$ 783 / \mathrm{kW}$ for stack costs. The 2011 energy related component was estimated at $\$ 215 / \mathrm{kWh}$. Fixed and variable O\&M costs for 2011 and 2020 were estimated at $\$ 5$ per $\mathrm{kW}$ and 0.1 cents/kWh, respectively. 
Table A.7. Summary of Capital Cost Diversity for Vanadium Redox Flow Battery Systems

\begin{tabular}{|c|c|c|c|c|c|}
\hline $\begin{array}{l}\text { Capital Cost } \\
(\$ / \mathrm{kW})\end{array}$ & $\begin{array}{l}\text { Capital Cost } \\
(\$ / \mathrm{kWh})\end{array}$ & $\begin{array}{l}\text { O\&M Fixed } \\
\text { (\$/kW-year) }\end{array}$ & $\begin{array}{c}\text { O\&M } \\
\text { Variable } \\
\text { (cents/kWh) }\end{array}$ & Efficiency (\%) & Reference \\
\hline \multirow[t]{2}{*}{1138 (Euros) $^{(\mathrm{a})}$} & 100 (Euros) & & & & (Joerissen et al. 2004) \\
\hline & & & & $72 \%-85 \%$ & $\begin{array}{l}\text { (Kaizuka and Sasaki } \\
\text { 2001; Tokuda 2000; } \\
\text { Rydh 1999; Zhao et al. } \\
\text { 2006; Eckroad 2007) }\end{array}$ \\
\hline $\begin{array}{l}4800 / 29600 \\
(2.5 \mathrm{MW}-10 / \\
100 \mathrm{MWh})\end{array}$ & $\begin{array}{l}\text { 1200/350 } \\
(2.5 \mathrm{MW}- \\
\text { 10/100 MWh) }\end{array}$ & $4.6^{(\mathrm{b})}$ & & & (Corey et al. 2002) \\
\hline $\begin{array}{l}1800 / 2600 \text { (4/8h) } \\
\text { (Euros) }\end{array}$ & & $\begin{array}{l}0.5 \% \text { capital } \\
\text { cost }\end{array}$ & & $65 \%-75 \%$ & (Staudt) \\
\hline $\begin{array}{l}\text { 1200/2000 } \\
(10 \mathrm{MWac} \\
\text { 30-100 MWh) }\end{array}$ & $\begin{array}{l}\text { 400-200 } \\
(10 \mathrm{MWac} \\
\text { 30-100 MWh) }\end{array}$ & $39^{(\mathrm{c})}$ & $0.2-0.7$ & $65 \%-75 \%$ & (Gyuk 2003b) \\
\hline 1620 & 217 & & & $72-90$ & (Kear 2011) \\
\hline $2300 / 1250$ & $300 / 210$ & & & & \\
\hline 970 (Euros) $^{(\mathrm{d})}$ & 78 (Euros) & & & $75 \%$ & (Jossen and Sauer 2006) \\
\hline \multicolumn{6}{|c|}{$\begin{array}{l}\text { (a) For } 2 \mathrm{~kW} 30 \mathrm{kWh} \text { and } 2 \mathrm{~kW} 300 \mathrm{kWh} \text { systems } \\
\text { (b) Actual O\&M cost was } \$ 20 / \mathrm{kW} \text {-year, but this may include annual property taxes and labor. For Na-S, O\&M } \\
\text { cost was } \$ 13 / \mathrm{kW} \text {-year. Use this ratio and multiply by O\&M cost for Na-S used in this report } \\
\text { (c) Includes annual property taxes and labor, which can be as high as } 2 \% \text { of capital costs } \\
\text { (d) For } 2 \mathrm{~kW} / 30 \mathrm{kWh} \text { system. Tank cost was included in their calculation - removed it }\end{array}$} \\
\hline
\end{tabular}

\section{A.7 Technology and Manufacturing Readiness Levels}

Table A.8 provides a short description of Technology Readiness Levels (TRL) (DOE 2009), while Table A.9 addresses MRL (DOD 2009).

Table A.8. Description of TRL

\begin{tabular}{|c|c|}
\hline TRL & Description \\
\hline TRL 1 & Basic Research \\
\hline TRL 2 & Applied Research \\
\hline TRL 3 & Critical Function or Proof of Concept Established \\
\hline TRL 4 & $\begin{array}{l}\text { Laboratory testing/Validation of } \\
\text { Component(s)/Process(es) }\end{array}$ \\
\hline TRL 5 & $\begin{array}{l}\text { Laboratory Testing of Integrated/Semi-Integrated } \\
\text { System }\end{array}$ \\
\hline TRL 6 & Integrated Pilot System Demonstrated \\
\hline TRL 8 & System Incorporated in Commercial Design \\
\hline TRL 9 & $\begin{array}{l}\text { System Proven and Ready for Full Commercial } \\
\text { Deployment }\end{array}$ \\
\hline
\end{tabular}


Table A.9. Description of MRL

\begin{tabular}{ll}
\hline \multicolumn{1}{c}{ MRL } & \multicolumn{1}{c}{ Description } \\
\hline MRL 1 & Manufacturing feasibility assessed \\
MRL 2 & Manufacturing concepts defined \\
MRL 3 & Manufacturing concepts developed \\
MRL 4 & Laboratory manufacturing process demonstration \\
MRL 5 & Manufacturing process development \\
MRL 6 & Critical manufacturing process prototyped \\
MRL 7 & Prototype manufacturing system \\
MRL 8 & Manufacturing Process Maturity Demonstration \\
MRL 9 & Manufacturing processes proven \\
MRL 10 & Full rate production demonstrated and lean production practices in \\
& place \\
\hline
\end{tabular}

\section{A.7.1 Fixed O\&M Cost}

The PCS consists of equipment necessary for energy transfer between the grid and energy storage system. The BOP is a catch-all for anything not covered by PCS. This includes project engineering, construction management, transformers for grid connection, land, foundation, building and other costs. The O\&M costs for BOP have not been included in this analysis, since such costs are expected to be uniform across all technologies. For Na-S batteries, fixed O\&M costs given in the literature varied over a wide range without any consistency on what services are included and which are excluded from the fixed O\&M cost. The low figures start at \$0.5/kW-year (Lamont 2004; Gyuk and Eckroad 2004) and go up to \$51/kW-year (Schoenung and Hassenzahl 2003; Gyuk and Eckroad 2003), which includes insurance and property taxes. For the purpose of this study, we used $\$ 3 / \mathrm{kW}$-year as the fixed O\&M cost, which corresponds to $\$ 0.46 / \mathrm{kWh}$ for E/P of 6.5. Since the E/P ratio for the batteries used varies, we have used $\$ 0.46 / \mathrm{kWh}$ as fixed O\&M cost in this report.

\section{A.7.2 Variable O\&M Cost}

Variable costs have traditionally been reported in cents/kWh, and ranged from 0.4-0.7 cents/kWh, where kWh is the cumulative energy out of the battery (Lamont 2004, Schoenung et al. 1996). The variable O\&M cost for Na-S systems for various applications ranged from \$2.6-\$13.4/kW-year (Gyuk and Eckroad 2003). An average of these values yields $\$ 7 / \mathrm{kW}$-year, while conversion of this to $\$ / \mathrm{kWh}$ yields $0.4-0.8$ cents $/ \mathrm{kWh}$. We used 0.7 cents per $\mathrm{kWh}$ in this study. Table A.10 summarizes the O\&M cost estimates as found in the literature. 
Table A.10. Summary of O\&M fixed and Variable Costs for Na-S Battery

\begin{tabular}{lll}
\hline \multicolumn{1}{c}{ Fixed O\&M (\$/kW-year) } & \multicolumn{1}{c}{ Variable O\&M } & \multicolumn{1}{c}{ Reference } \\
\hline 3 & $16.9(\$ / \mathrm{kW}$-year) & (Gyuk and Eckroad 2004) \\
20 & & (Schoenung and Hassenzahl 2003) \\
$13-51(\$ 2 / \mathrm{kW}$-year for PCS) & $2.6-13.4(\$ / \mathrm{kW}$-year) & (Gyuk and Eckroad 2003) \\
0.5 & 0.7 cents $/ \mathrm{kWh}$ & (Lamont 2004) \\
1.5 & 0.5 cents $/ \mathrm{kWh}$ & (Schoenung et al. 1996) \\
$\mathbf{\$ 0 . 4 6 / k W h}$ & $\mathbf{0 . 7}$ cents $/ \mathbf{k W h}$ & Selected for study \\
\hline
\end{tabular}

\section{A.7.3 Efficiency}

For Na-S batteries, the AC-AC efficiency was in the range of 0.75-0.85 (Kishinevsky 2006;

Schoenung and Hassenzahl 2003; Schoenung 2001; Technology Insights 2005). We chose a roundtrip efficiency of 0.78 . For the Li-ion battery, the efficiency was estimated to be 0.8 (Rastler et al. 2007). These figures correspond to battery system efficiencies that include all of the losses in the PCS. The specific PCS efficiency was estimated to be 0.95 . The roundtrip efficiency is expected to change as a function of charge and discharge rate. For this analysis, the efficiency is kept constant for all rates. 

Appendix B

Specific Operational Strategies to Meet Balancing Requirements 



\section{Appendix B}

\section{Specific Operational Strategies to Meet Balancing Requirements}

To explore different operational strategies and how their selection will impact balancing requirements, several technology cases were introduced in Section 3.2. Table 3.4 provided a brief outline of the different cases, and is replicated on the next page as Table B.1 for convenience. A more detailed description of each case in Table B.1 is presented in the sections of this appendix.

\section{B.1 Case 1: Combustion Turbines}

The base case for operational strategies involves the use of only CTs for energy balancing requirements. Part load efficiencies are considered in the CT implementations. This scenario represents a case similar to current operational procedures.

The size for the combustion turbine is set by the requirements for generation increment, not the sum of increment and decrement. This is based on the notion that the existing combustion turbine capacity is operating at the zero balancing point already and would be able to provide generation decrements.

\section{B.2 Case 2: Na-S batteries + CC}

The second scenario utilizes Na-S batteries and CC generation to meet balancing requirements. Figure B.1 shows the typical power output of the Na-S battery storage and CC generator over a two-day period. CC generation is used to compensate for the efficiency loss of the batteries, and to provide a constant energy source for the batteries to assure a net zero energy change over the course of the entire day. As such, the Na-S contributions are actually the difference between the blue line and red line at each interval. If above the red line, the Na-S battery is discharging into the system. If below the red line, the storage is charging. 
Table B.1. Definition of Technology Cases

\begin{tabular}{|c|c|c|c|}
\hline & Case & Technology & Comments \\
\hline \multirow{9}{*}{ 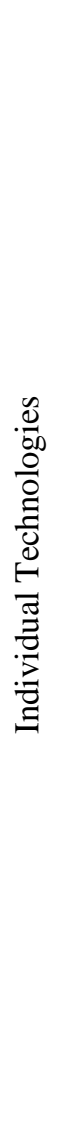 } & $\mathrm{C} 1$ & Combustion turbine & Conventional technology considered as the reference case \\
\hline & $\mathrm{C} 2$ & $\mathrm{Na}-\mathrm{S}$ & Sodium sulfur battery only \\
\hline & C3 & Li-ion & Lithium-ion battery only \\
\hline & $\mathrm{C} 4$ & Flywheel & Flywheel only \\
\hline & C5 & CAES with 2 mode changes & $\begin{array}{l}\text { CAES with a 7-minute waiting period for mode changes } \\
\text { (compression-generation and vice versa). Balancing services } \\
\text { will be provided during compression mode at night (8pm-8am) } \\
\text { and during generation mode during the day (8am-8pm). Na-S } \\
\text { battery is assumed to make up operations during } 7 \text { minute } \\
\text { waiting period. }\end{array}$ \\
\hline & C6 & Flow battery & Flow battery only \\
\hline & C7 & PH with multiple mode changes & $\begin{array}{l}\text { Pumped hydro with a 4-minute waiting period for mode changes } \\
\text { (pumping-generation and vice versa). This machine allows to } \\
\text { multiple mode changes during the day. Na-S battery is assumed } \\
\text { to make up operations during } 4 \text { minute waiting period. }\end{array}$ \\
\hline & $\mathrm{C} 8$ & PH with 2 mode changes & $\begin{array}{l}\text { Same as (C7), except only two mode changes. Balancing } \\
\text { services will be provided during pumping mode at night (8pm- } \\
\text { 8am) and during generation mode during the day (8am-8pm). } \\
\text { Na-S battery is assumed to make up operations during } 4 \text { minute } \\
\text { waiting period. }\end{array}$ \\
\hline & C9 & DR & $\begin{array}{l}\text { Demand response only. This assumes that balancing services } \\
\text { will be provided as a load. Only considered is PHEV charging } \\
\text { at home and work. Resources are expressed in MW of DR } \\
\text { capacity as well as in numbers of PHEV with demand response } \\
\text { capability. }\end{array}$ \\
\hline \multirow{8}{*}{ 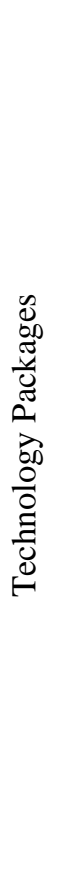 } & $\mathrm{C} 10$ & $\mathrm{Na}-\mathrm{S}$ & Sodium sulfur hattery and DR combined \\
\hline & & DR & sodium sultur battery and DR combined \\
\hline & C11 & $\begin{array}{l}\text { Li-ion } \\
\text { DR }\end{array}$ & Lithium-ion battery and DR combined \\
\hline & $\mathrm{C} 12$ & $\begin{array}{l}\text { CAES } \\
\text { Flywheel }\end{array}$ & $\begin{array}{l}\text { CAES with no constraints for mode changes with Flywheel. The } \\
\text { balancing requirement is allocated to each technology according } \\
\text { to minimum cost. }\end{array}$ \\
\hline & C13 & $\begin{array}{l}\text { PH with multiple mode chances } \\
\text { Na-S }\end{array}$ & $\begin{array}{l}\text { Pumped hydro with no constraints for mode changes with } \mathrm{Na}-\mathrm{S} \\
\text { battery. The balancing requirement is allocated to each } \\
\text { technology according to minimum cost. }\end{array}$ \\
\hline & C14 & $\begin{array}{l}\text { PH with } 2 \text { mode changes } \\
\text { Na-S }\end{array}$ & $\begin{array}{l}\text { Pumped hydro with two mode changes per day (see C8) with } \\
\text { Na-S battery. The balancing requirement is allocated to each } \\
\text { technology according to minimum cost. }\end{array}$ \\
\hline & C15 & $\begin{array}{l}\text { PH with multiple mode chances } \\
\text { Flywheel }\end{array}$ & $\begin{array}{l}\text { Pumped hydro with no constraints for mode changes with } \\
\text { Flywheel. The balancing requirement is allocated to each } \\
\text { technology according to minimum cost. }\end{array}$ \\
\hline & C16 & $\begin{array}{l}\text { PH with } 2 \text { mode changes } \\
\text { Flywheel }\end{array}$ & $\begin{array}{l}\text { Pumped hydro with two mode changes per day (see C8) with } \\
\text { Flywheel. The balancing requirement is allocated to each } \\
\text { technology according to minimum cost. }\end{array}$ \\
\hline
\end{tabular}




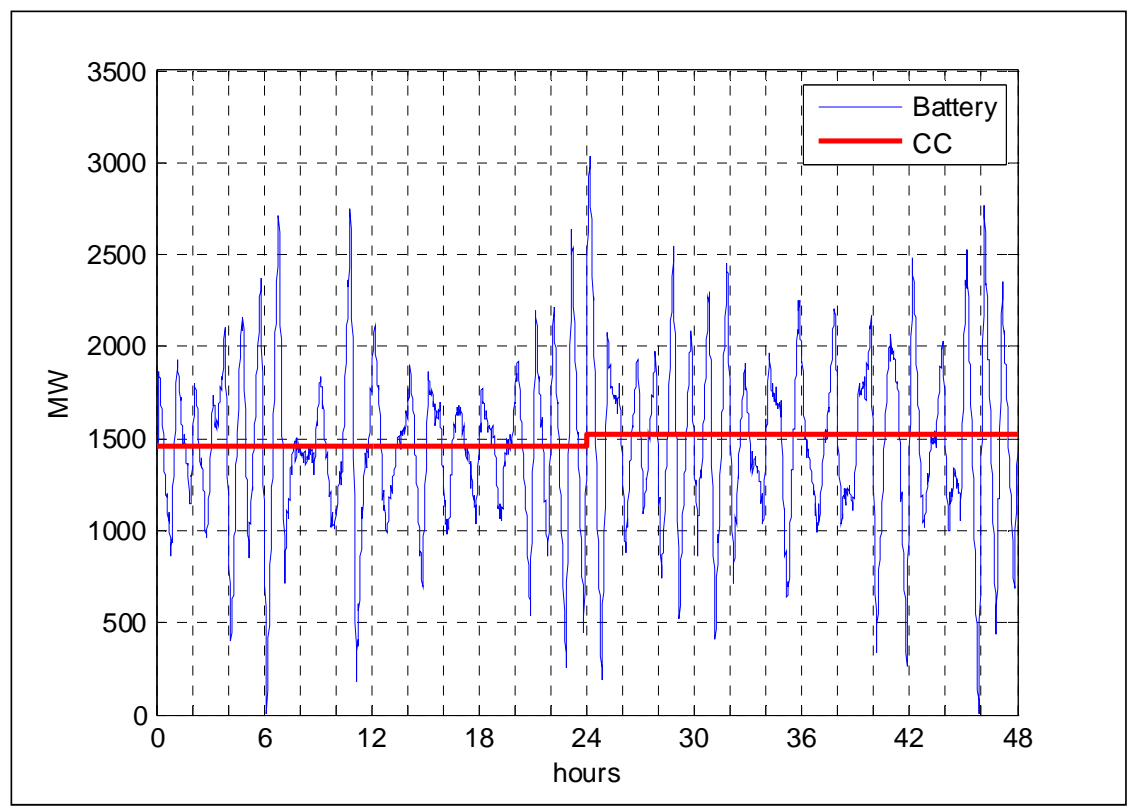

Figure B.1. Power Output of Na-S Battery and CC Generation for Two-Day Period

\section{B.3 Case 3: Li-Ion + CC}

The third scenario focuses on the use of Li-ion batteries and CC generation. The scenario is executed in an identical manner to Case 2 above, but the lower efficiency Na-S batteries are replaced with Li-ion batteries. CC generation is once again utilized to compensate for efficiency losses in the battery storage and to ensure a balanced energy transfer over the day. The efficiency of Li-ion batteries was nearly identical to that of Na-S batteries for this case (80\% compared to 78\%). The typical power output of Figure B. 1 is also representative of the Li-ion battery and CC case.

\section{B.4 Case 4: Flywheel + CC}

The fourth scenario focuses on the use of flywheels and CC generation. The scenario is executed in an identical manner to Case 2 above, but the lower efficiency Na-S batteries are replaced with flywheels. CC generation is once again utilized to compensate for efficiency losses in the flywheels and to ensure a balanced energy transfer over the day. The efficiency of flywheel was higher than that of Na-S batteries (90\% compared to 80\%). The typical power output of Figure B.1 is also representative of the Flywheel and CC.

\section{B.5 Case 5: CAES with Two Mode Changes + CC}

The CAES is restricted to two mode changes (changes between compression-generation and vice versa) per day. The CAES operates in compression mode from 8 p.m. to 8 a.m., and operates in generation mode from 8 a.m. to 8 p.m. each day. A 7-minute changeover delay is incorporated into the CAES system. This changeover delay is handled by supplementary Na-S battery storage. CC generation is not only utilized to compensate for efficiency losses in the battery and CAES, but also to provide additional compressing power. Figure B.2 represents the power output of the CAES storage when 
restricted to only two operating modes. The areas associated with the Na-S storage are not visible on this plot, as they only represent 14 minutes out of the 24-hour period.

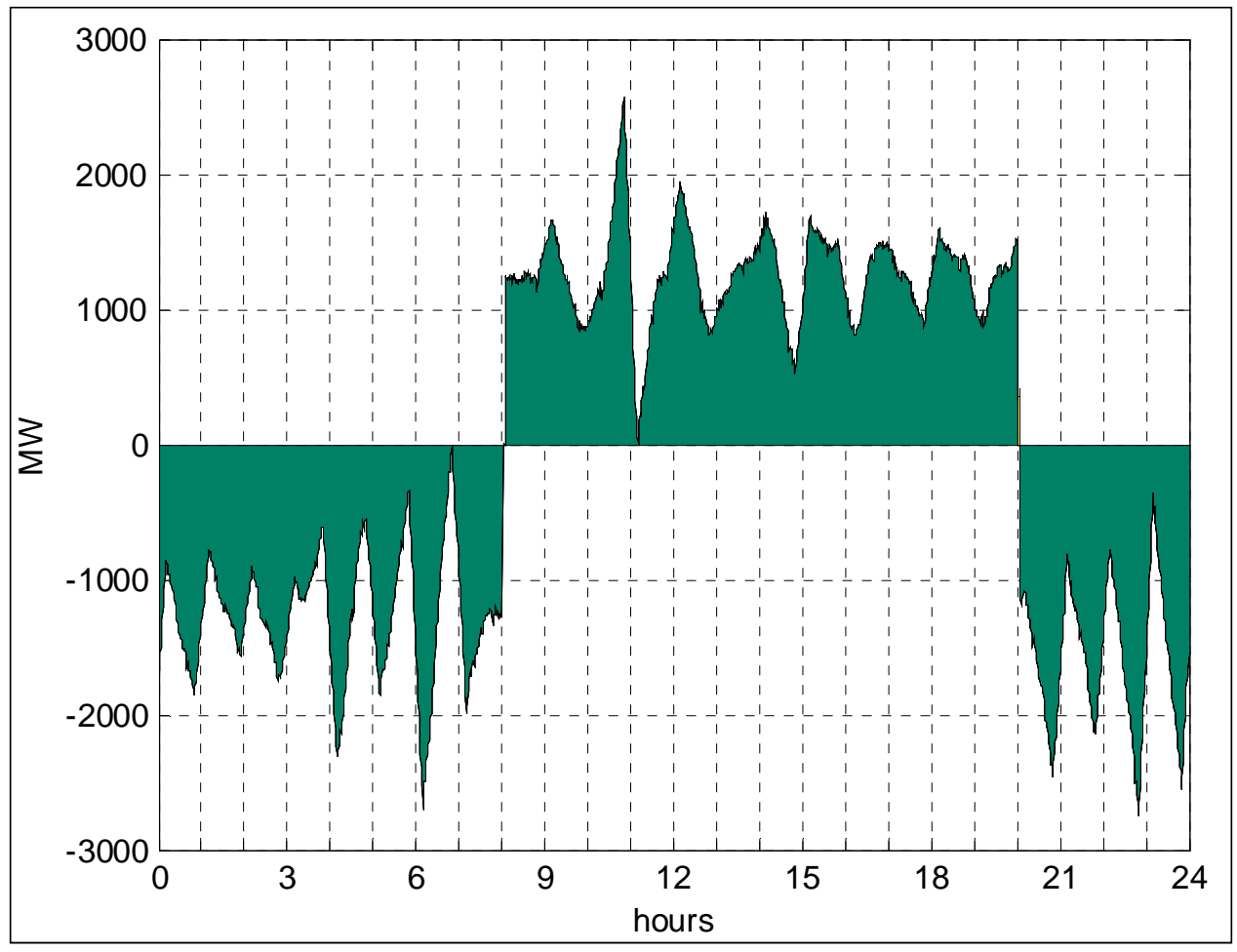

Figure B.2. Power Output of CAES with Only Two Mode Changes Per Day

\section{B.6 Case 6: Flow battery + CC}

The sixth scenario focuses on the use of flow battery and CC generation. The scenario is executed in an identical manner to Case 2 above, but the higher efficiency Na-S batteries are replaced with flow batteries. CC generation is once again utilized to compensate for efficiency losses in the flow batteries and to ensure a balanced energy transfer over the day. The efficiency of flow battery was lower than that of Na-S batteries (75\% compared to 80\%). The typical power output of Figure B.1 is representative of the flow battery and CC case as well.

\section{B.7 Cases 7: Pumped Hydro with Multiple Mode Changes + CC}

Technology Case 7 utilizes pumped hydro generation for the primary balancing requirement. For this particular case, the pumped hydro has no mode switching limit. The pumped hydro storage can switch between pumping and generation modes as many times as necessary during the day. This results in approximately 40 mode changes a day, which can cause a considerable drop in the expected lifetime of the equipment (Spitzer and Penninger 2008). Mode changes experience a 4-minute changeover delay. During the changeover, Na-S batteries are utilized to cover the balancing requirements. Figure B.3 demonstrates this implementation. As with the previous cases, CC generation is utilized to compensate for the efficiency losses of both the Na-S battery and pumped hydro, as well as balance the energy consumption in the storage. 


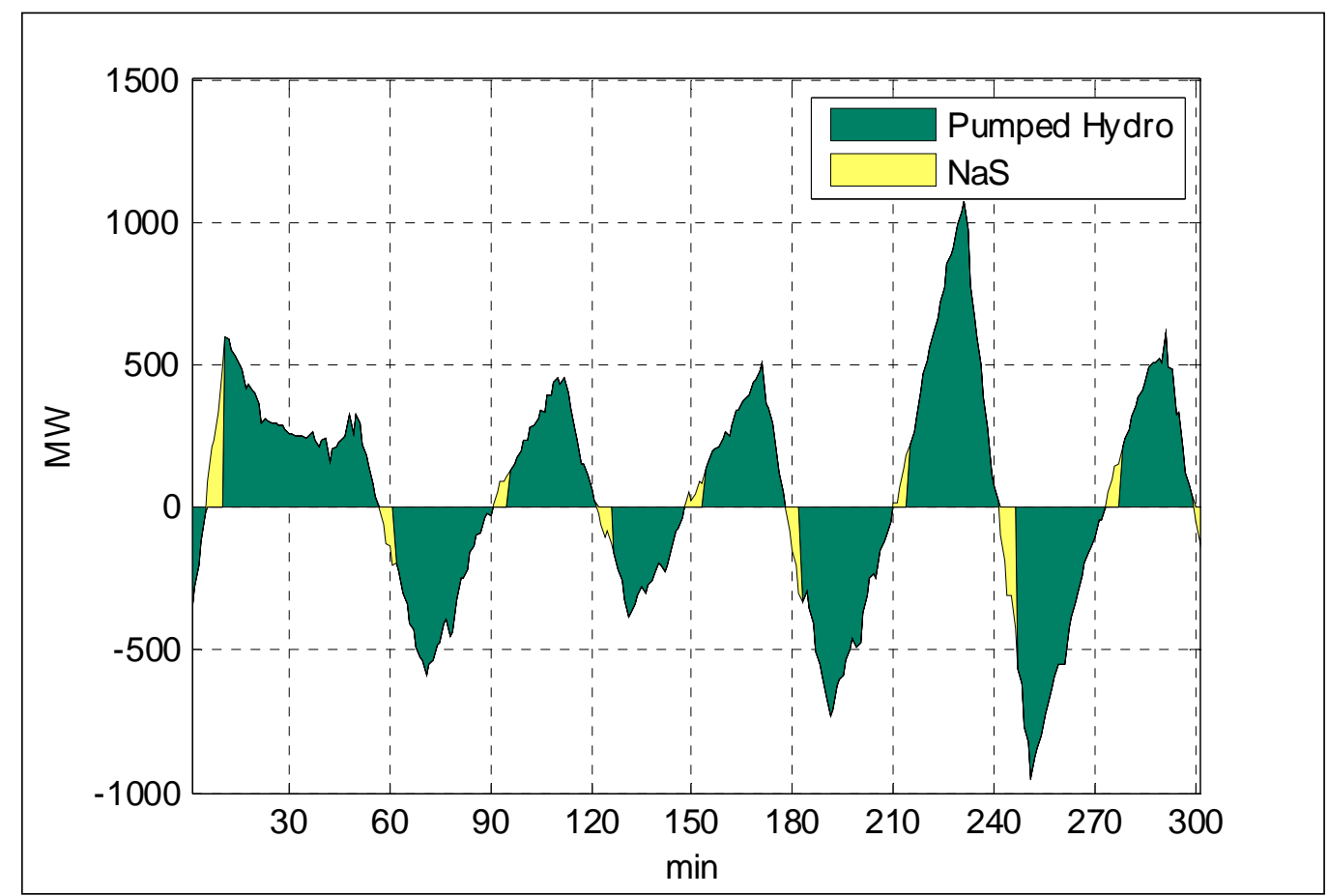

Figure B.3. Balancing Signal Taken by Pumped Hydro and Na-S Battery When the Changeover Delay is Modeled

\section{B.8 Case 8: Pumped Hydro with Two Mode Changes + CC}

Technology Case 8 is very similar to the scenario in Case 7. However, the pumped hydro storage is restricted to two mode changes per day. The pumped hydro operates in pump mode from 8 p.m. to 8 a.m., and operates in generation mode from 8 a.m. to 8 p.m. each day. This reduced number of mode changes increases the expected lifetime of the equipment, when compared to Case 7 . As with Case 7, a 4-minute changeover delay is incorporated into the pumped hydro system. This changeover delay is again handled by supplementary Na-S battery storage. CC generation is not only utilized to compensate for efficiency losses in the battery and pumped hydro storage, but also to provide additional pumping power. Figure B.4 represents the power output of the pumped hydro storage when restricted to only two operating modes. The yellow areas associated with the Na-S storage are not visible on this plot, as they only represent 8 minutes out of the 24-hour period. 


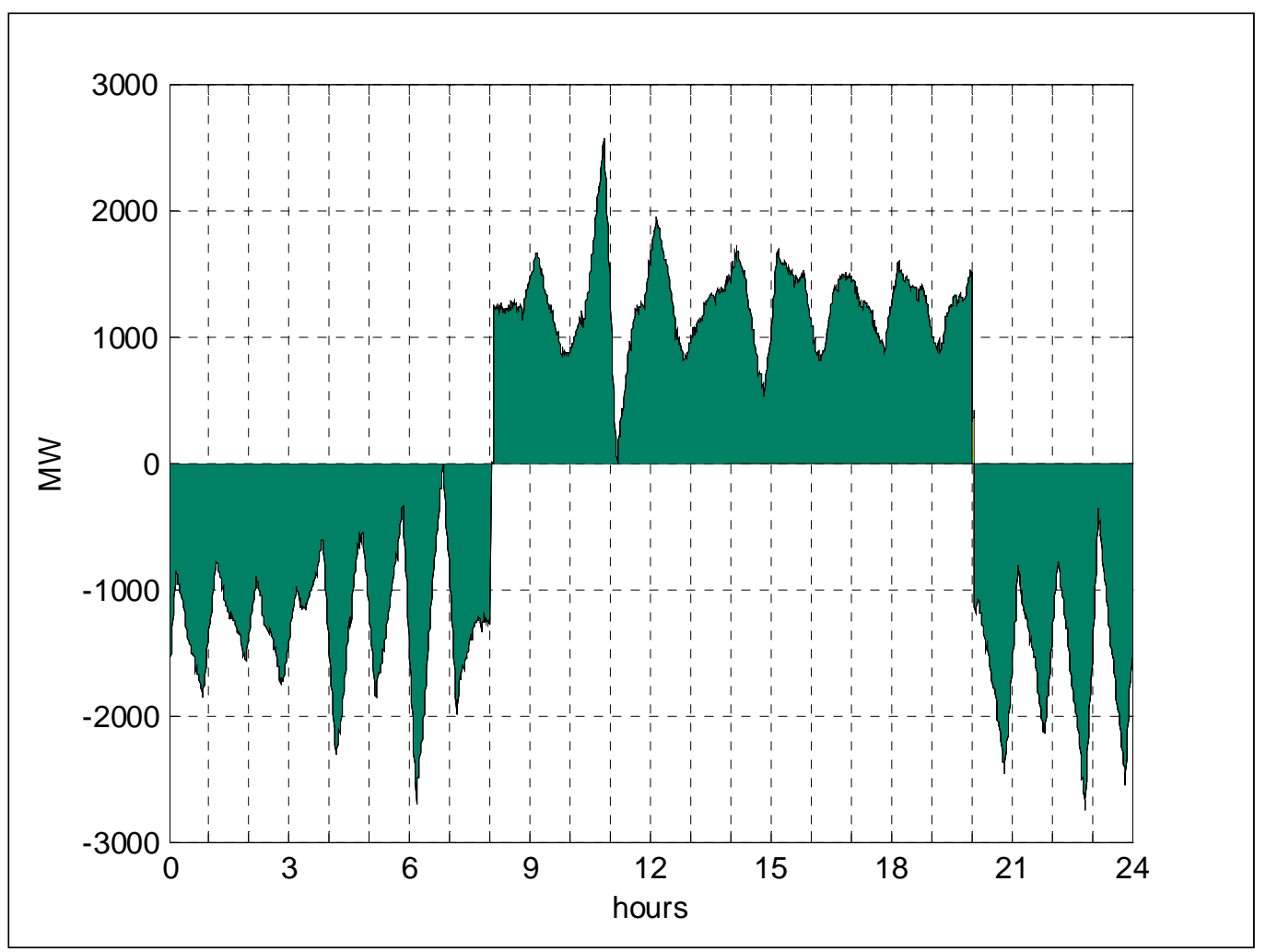

Figure B.4. Power Output of Pumped Hydro with Only Two Mode Changes Per Day

\section{B.9 Case 9: Demand Response}

Technology Case 9 utilizes a different scenario to meet the balancing requirements. Using DR, the load of the system is adjusted to meet the varying energy demands of the system, rather than using an energy storage solution. Pure DR balancing was accomplished using PHEV charging where both home and work charging was assumed available.

Figure B.5 shows the balancing signal and the load resource availability of EVs. The balancing would be achieved solely during the charging mode. No Vehicle-to-Grid (V2G) is necessary to meeting the balancing requirements. The balancing services can be furnished only during the charging mode. PNNL coined the term V2Ghalf, expressing the feature of intelligent or smart charging whereby the balancing is provide by a load resource (i.e., charging of a EV/PHEV battery) in such a manner that the charging is varied around an operating point. The aggregated EV battery charging load is not constant but varies as a function of time-of-day and availability of public charging stations at the workplace to allow for making the vehicle resource available to grid services. The number of vehicles necessary to provide sufficient load resources is then the number of vehicles that will furnish just enough load to meet the maximum balancing capacity, as seen in Figure B.4, at 6:00 a.m. when most of the chargers are turned off after having recharged the battery overnight. 


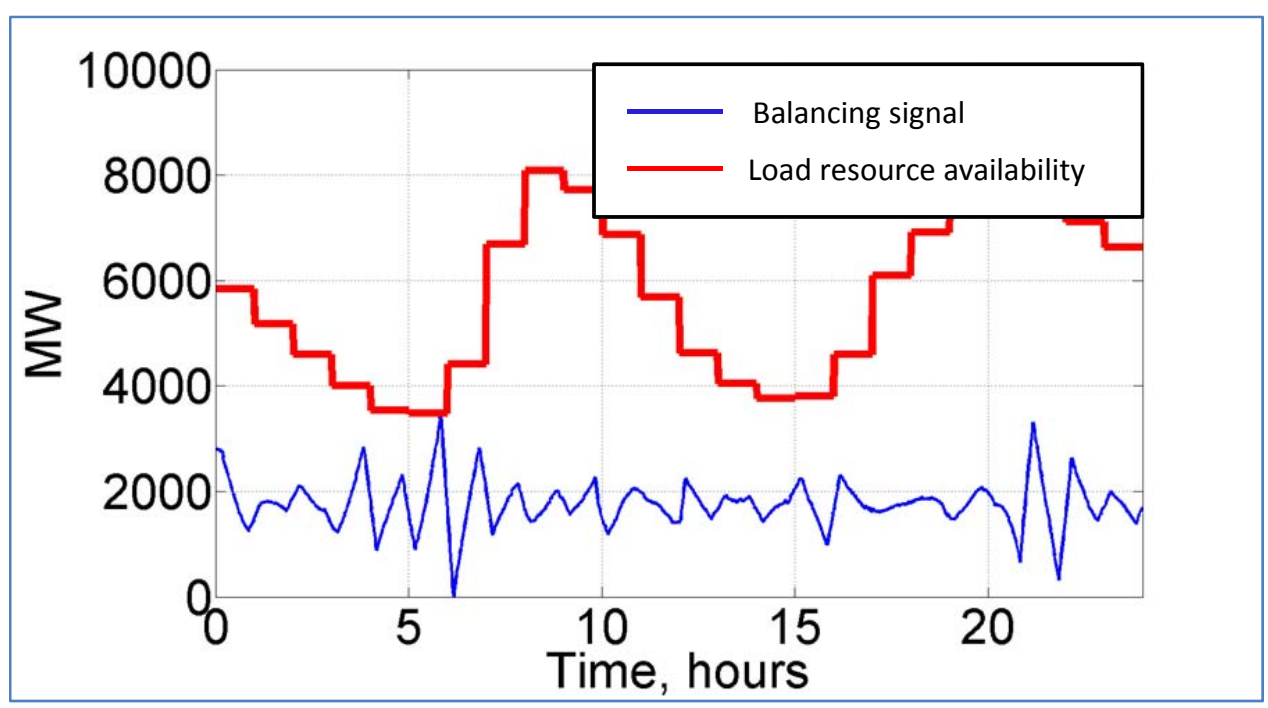

Figure B.5. Load Curves for PHEV with Home and Work Charging and Balancing Signal for the Average PHEV

Once a proper battery size was obtained, the vehicle's current SOC was randomized. As part of this randomization, it is often necessary to remove the first day of simulation results from each parameter investigation. This first day is often used to initialize the population into its charging routine, so some abnormal behavior is often present. Figure B.6 shows the first three days of a simulation investigating a particular ratio of home-only and work-home charging. While variations in the individual days are expected (due to the nature of the balancing signal), the first half day is noticeably different.

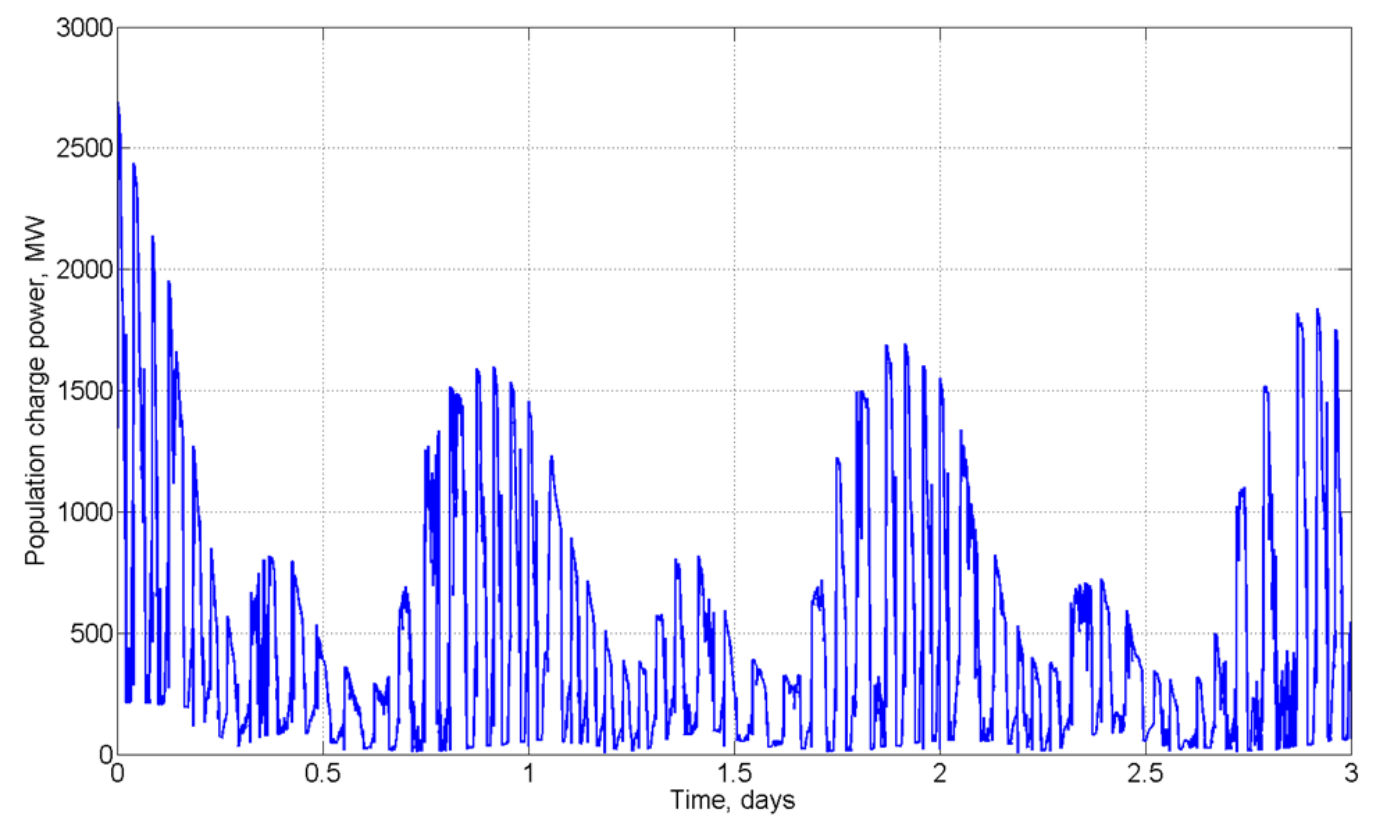

Figure B.6. Load Curves Population Charge Rate over Three-Day Period 
It should also be noted that vehicle battery sizes and states of charge are calculated as the fully available capacity. That is, a 3.0-kWh battery is assumed to have all $3.0 \mathrm{kWh}$ of energy available for use. The industry practice of keeping a battery in an optimal SOC band (i.e., 25\% to 90\% (Tate et al. 2008) to extend life is not utilized here. One can reasonably assume that the battery capacities mentioned could merely be an "adjusted battery size." That is, the 3.0-kWh battery is really a 4.62-kWh battery, but only $3.0 \mathrm{kWh}$ is normally available for use.

\section{B.10 Case 10: CC + Na-S + DR}

The tenth technical case utilizes a combination of CC generation, Na-S battery storage, and DR to meet the balancing requirements. CC generation is again utilized for energy balance, as well as compensating for battery efficiency.

The balancing requirements were divided between the Na-S storage and DR. Twelve combinations of "slow storage" (DR) and "fast storage" (Na-S) components were defined, including the extreme cases of one single technology. The lower frequency contents of the intra-hour balancing signal are assigned to the "slow storage" component, while the higher frequency contents of the intra-hour balancing signal are assigned to the other component ("fast storage”). The 12 technology shares are defined using the filtering process discussed in Section 4.3.

DR capabilities were modeled as PHEV-home and work charging as explained in the previous technology case. Supplemented with Na-S battery storage, the amount of DR required is changes according to the division of the balancing signal that defines the 12 technology shares.

To determine the optimal combination, the 12 technology shares are further optimized using the economic procedure discussed in Section 5. The full process is explained in Section 4.3.

\section{B.11 Case 11: CC + Li-ion + DR}

This case is similar to Case 10 discussed in the previous subsection; there is only a difference in the battery efficiency.

\section{B.12 Case 12: Flywheel + CC + CAES with Two Mode Changes}

The CAES was restricted to a night pump and day generation cycle, as per Case 5. However, the flywheel storage capacity was used instead of Na-S batteries. CC generation is again utilized for energy balance, as well as compensating for battery efficiency.

The balancing requirements were divided between the flywheel storage and CAES. Twelve combinations of "slow storage" (CAES) and "fast storage" (Flywheels) components were defined, including the extreme cases of one single technology (notice that CAES only still requires flywheels to make up operation during changeover waiting period). The lower frequency contents of the intra-hour balancing signal are assigned to the "slow storage" component, while the higher frequency contents of the intra-hour balancing signal are assigned to the other component ("fast storage"). The 12 technology shares are defined using the filtering process discussed in Section 4.3. 
Figure B.7 shows the power output of the CAES for one of the 12 technology shares. Figure B.8 shows the power output for the flywheel storage. The addition of the flywheel storage helps alleviate the amount of CAES required. Figures B.7 and B.8 change according to the division of the balancing signal that defines the 12 technology shares.

To determine the optimal combination, the 12 technology shares are further optimized using the economic procedure discussed in Section 5. The full process is explained in Section 4.3.

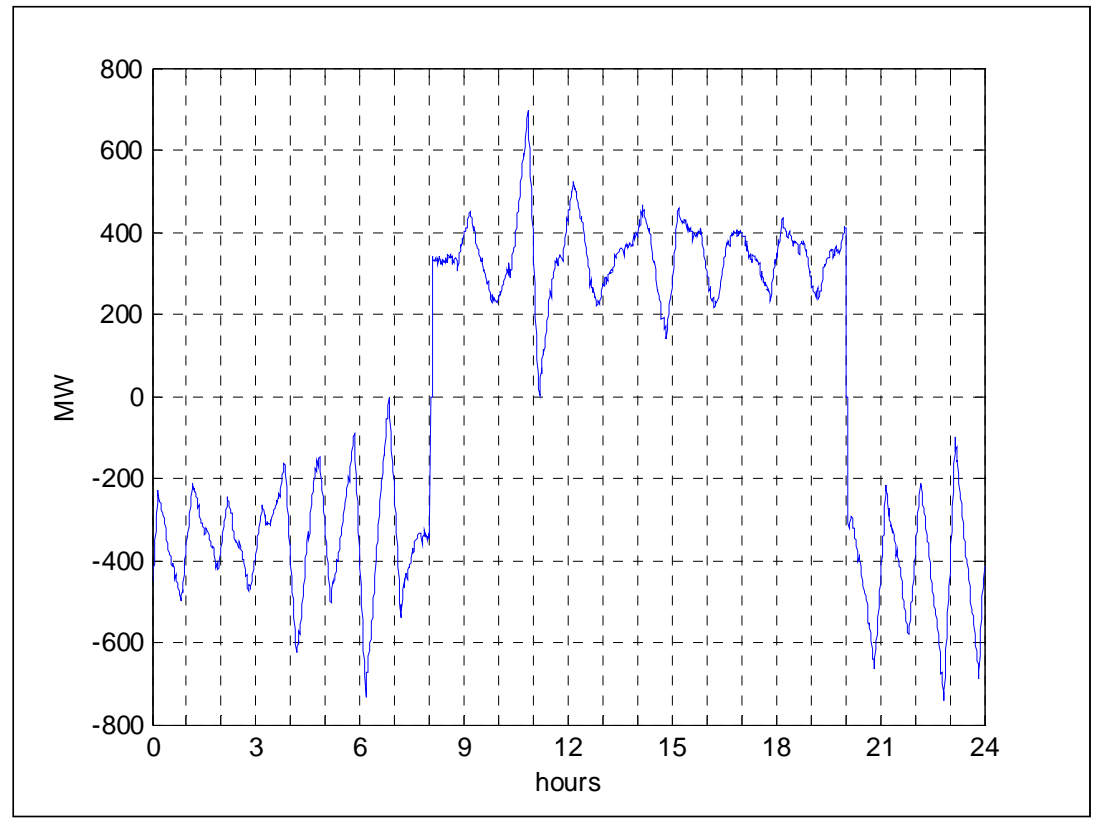

Figure B.7. Power Output of CAES with Only Two Mode Changes per Day for One of Twelve Technology Shares Options

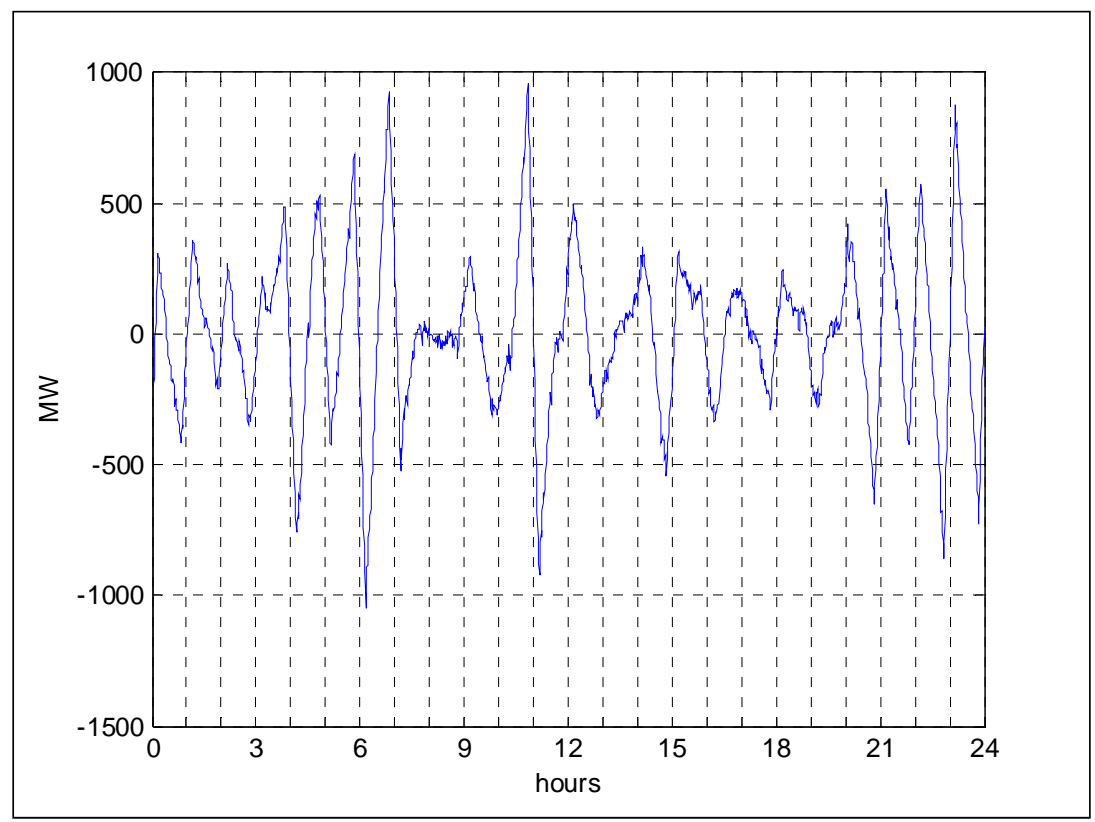

Figure B.8. Power Output of Flywheel for One of Twelve Technology Shares Options 


\section{B.13 Case 13: Na-S + CC + Pumped Hydro with Multiple Mode Changes}

Technology Case 7 earlier utilized pumped hydro storage with multiple mode changes in a day, which was supplemented by Na-S battery storage. This technology case supplements that analysis with a larger amount of Na-S battery storage available. Unlike Case 7, the balancing requirements are divided between the Na-S and pumped hydro storage. CC generation is again utilized for energy balance, as well as compensating for battery efficiency.

The balancing requirements were divided between the Na-S battery storage and CAES. Twelve combinations of "slow storage" (pumped hydro) and "fast storage" (Na-S) components were defined, including the extreme cases of one single technology (notice that pumped hydro only still requires Na-S to make up operation during changeover waiting period). The lower frequency contents of the intra-hour balancing signal are assigned to the "slow storage" component, while the higher frequency contents of the intra-hour balancing signal are assigned to the other component ("fast storage"). The 12 technology shares are defined using the filtering process discussed in Section 4.3.

Figure B.9 shows the power output of the pumped hydro for one of the 12 technology shares. Figure B.10 shows the power output for the Na-S battery storage. The addition of the flywheel storage helps alleviate the amount of pumped hydro required. Figures B.9 and B.10 change according to the division of the balancing signal that defines the 12 technology shares.

To determine the optimal combination, the 12 technology shares are further optimized using the economic procedure discussed in Section 5. The full process is explained in Section 4.3.

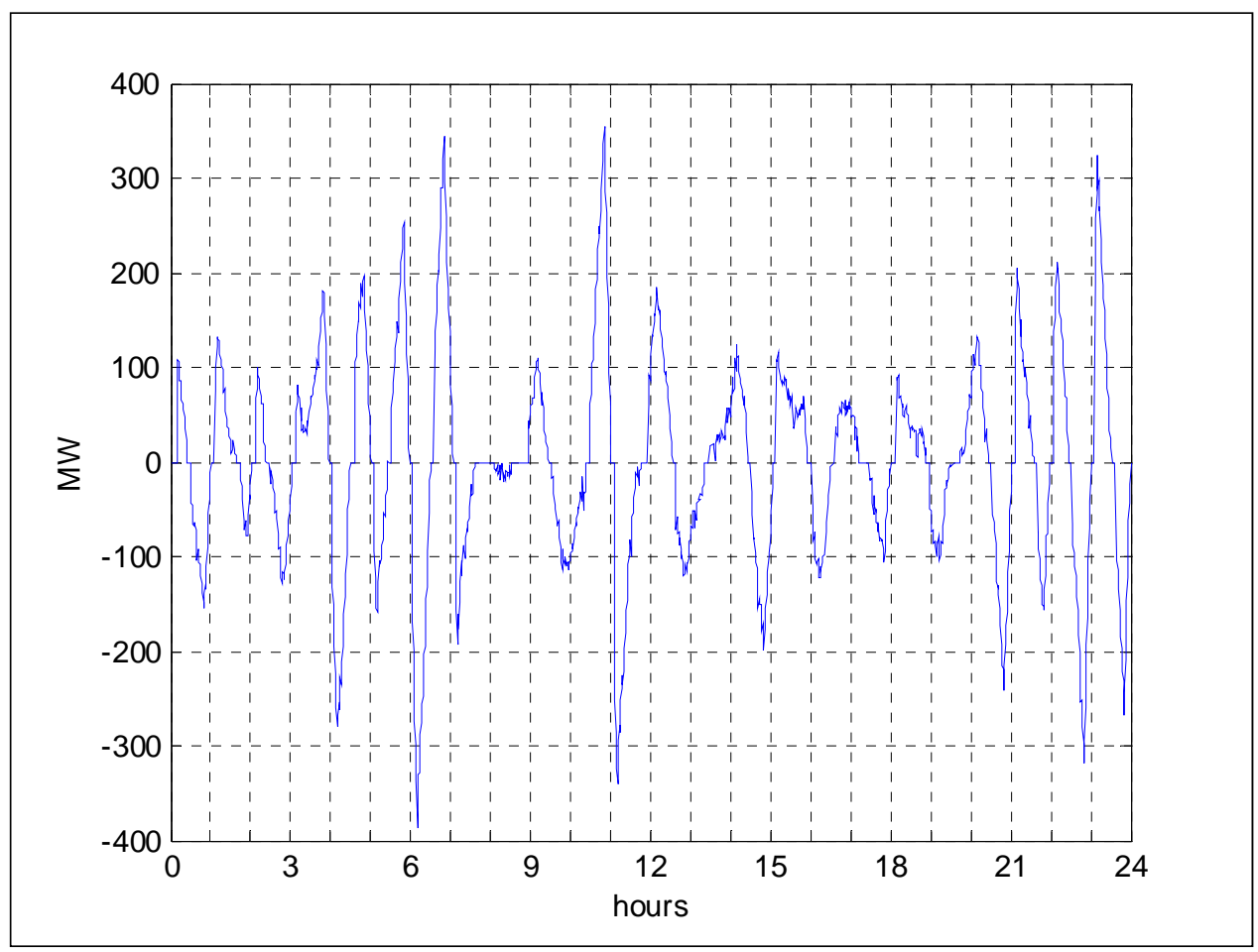

Figure B.9. Power Output of Pumped Hydro with Multiple Mode Changes per Day with Na-S + CC Technology Shares Options 


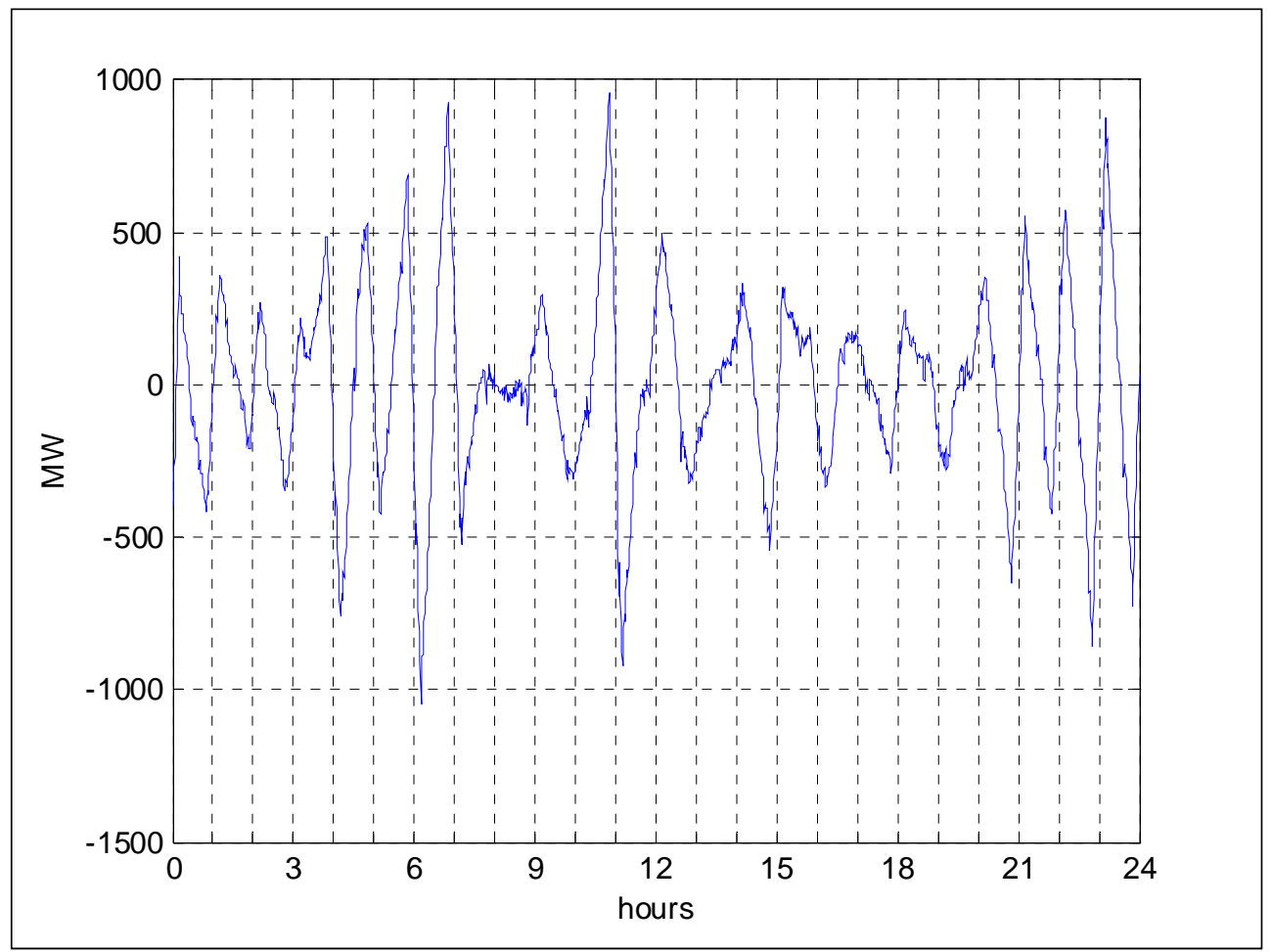

Figure B.10. Power Output of Na-S Battery for Na-S + Pumped Hydro + CC Scenario Technology Shares Options

\section{B.14 Case 14: Na-S + CC + Pumped Hydro with Two Mode Changes}

The pumped hydro and Na-S battery technology Case 8 was re-evaluated with various amounts of Na-S storage available. The procedure is similar to Case 12; there are the following differences: the storage efficiencies, the "slow storage" corresponds to pumped hydro, and the "fast storage" corresponds to the Na-S battery.

\section{B.15 Case 15: Pumped Hydro + Flywheel + CC + DR}

Case 15 considers pumped hydro with multiple mode changes and flywheel storage. Various combinations of these two technologies are evaluated. The procedure is similar to Case 13; there are the following differences: the storage efficiencies, the "slow storage" corresponds to pumped hydro storage, and the "fast storage" corresponds to the flywheel storage.

\section{B.16 Case 16: Pumped Hydro + Flywheel + CC + DR}

Case 16 considers pumped hydro with 2 mode changes and flywheel storage. Various combinations of these two technologies are evaluated. The procedure is similar to Cases 14 and 12; there are the following differences: the storage efficiencies, the "slow storage" corresponds to pumped hydro storage, and the "fast storage" corresponds to the flywheel storage. 


\section{B.17 Wind Spillage}

The balancing requirements are produced when there is over-generation and under-generation in the system. Balancing requirements caused by over-generation can be compensated using wind spillage, that is, wind generators can be controlled to generate less power than the available wind resource would allow. This action is called wind spillage in this study. Wind spillage can curtail the peaks of the overgeneration balancing signal. However, as it is illustrated in this section, wind spillage does reduce the capacity requirements (MW) slightly, but increases the energy capacity requirements (if energy storage is used). This is a non-intuitive result and is explained below.

Wind spillage introduces asymmetry to the balancing signal, since the peaks curtailments are only performed when there is over-generation and the under-generation peaks remain unchanged. As a result, the power requirements for balancing services are not considerably reduced. What is more, the energy requirements for balancing services increase due to the introduction of asymmetry in the balancing signal. An illustration of this phenomenon is given in this section.

Consider the case where the balancing requirements are meet by Na-S batteries only (Case 2). If the complete over-generation part of the balancing signal is curtailed by wind spillage, the balancing signal taken by the Na-S batteries is as shown in Figure B.11. Charging and discharging of the Na-S batteries is decided by the difference between the balancing signal and a daily fixed power output of a CC generator as shown in Figure B.12. It can be seen from Figure B.12 that the maximum power requirement for Na-S batteries (difference between balancing signal and constant power output of CC) is not considerably reduced by curtailing half of the balancing signal using wind spillage. What is more, the energy requirements for Na-S batteries are larger than the energy requirements without wind spillage as it can be seen comparing Figure B.13 and Figure B.14.

Figure B.15 and Figure B.16 show the energy and power requirements for several levels of wind spillage. A $100 \%$ wind spillage means that the complete over-generation side of the balancing signal is curtailed by wind spillage. It can be seen in Figure B.15 and Figure B.16 that wind spillage increases the energy requirements while it does not considerably reduces the power requirements. Therefore wind spillage by itself is not a good strategy to provide balancing services.

A reduction in the energy balancing requirements through wind spillage could be only achieved if the balancing signal is also curtailed in the under-generation peaks. DR can be used to curtail undergeneration peaks. Symmetry in the balancing signal can be maintained by using both DR and wind spillage. 


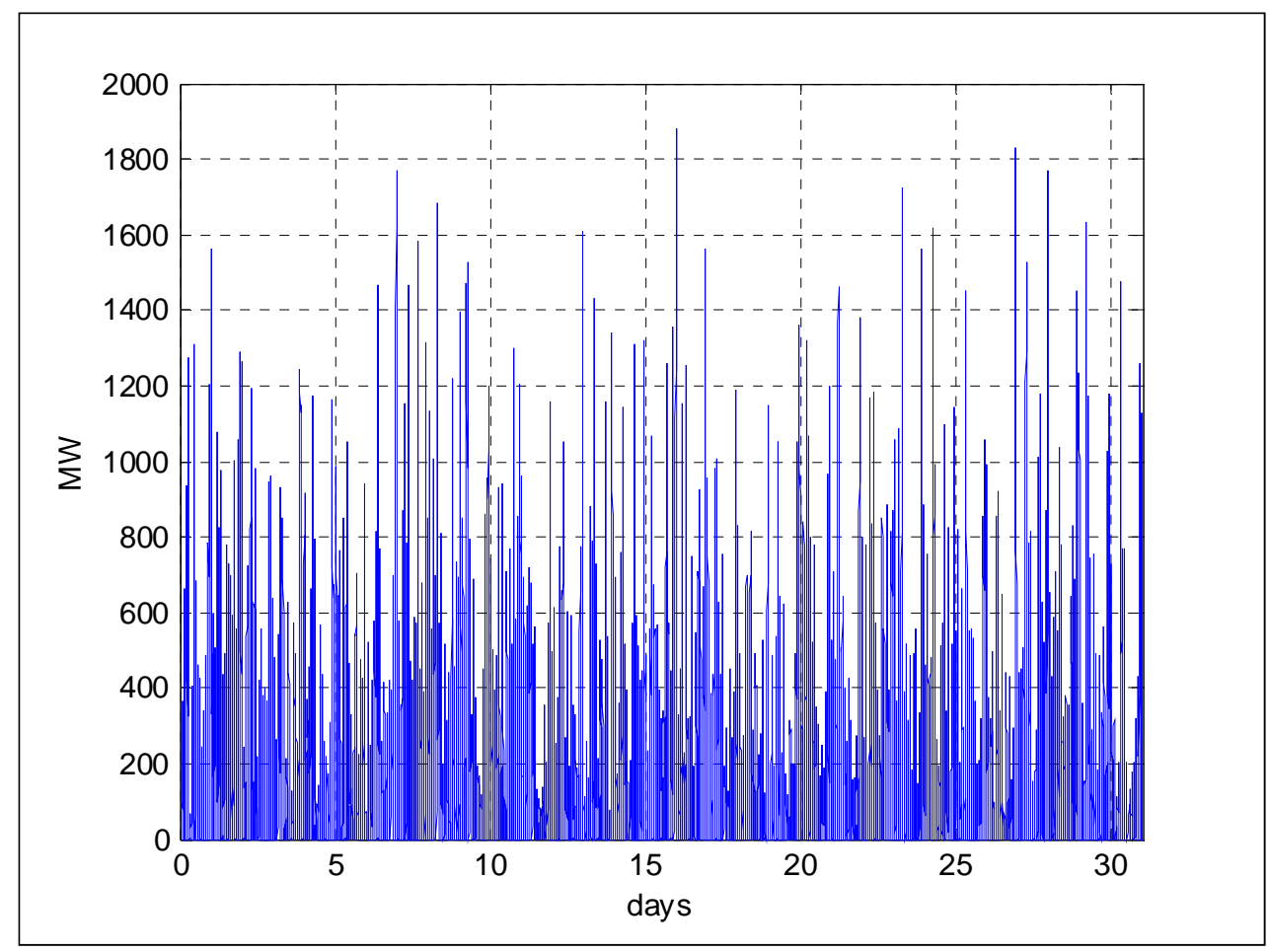

Figure B.11. Balancing Signal Taken by Na-S Batteries After the Complete Over-Generation Component is Curtailed by Wind Spillage

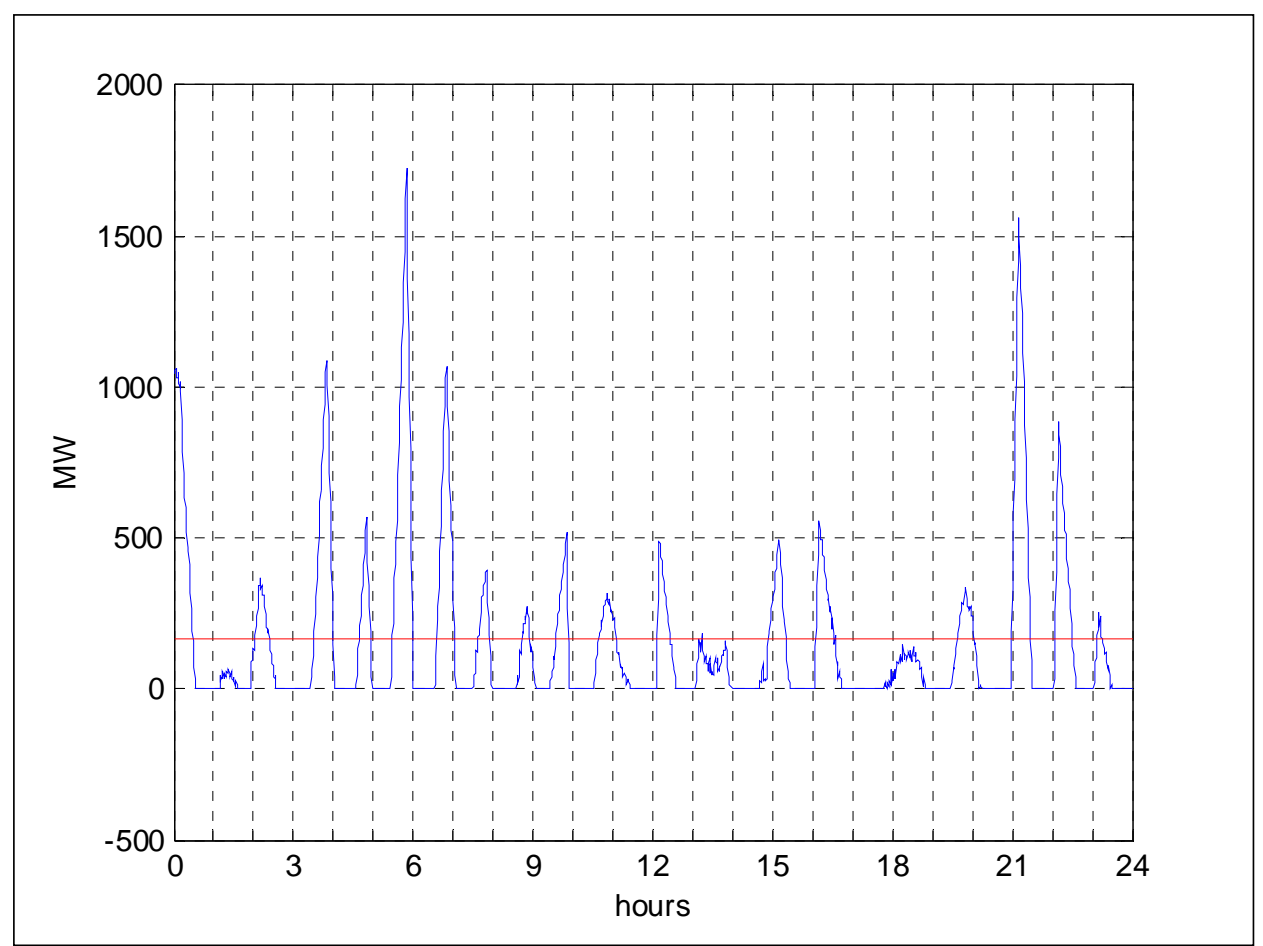

Figure B.12. Balancing Signal Taken by Na-S Batteries After the Complete Over-Generation Component is Curtailed by Wind Spillage, and Constant Power Output of CC Generation 


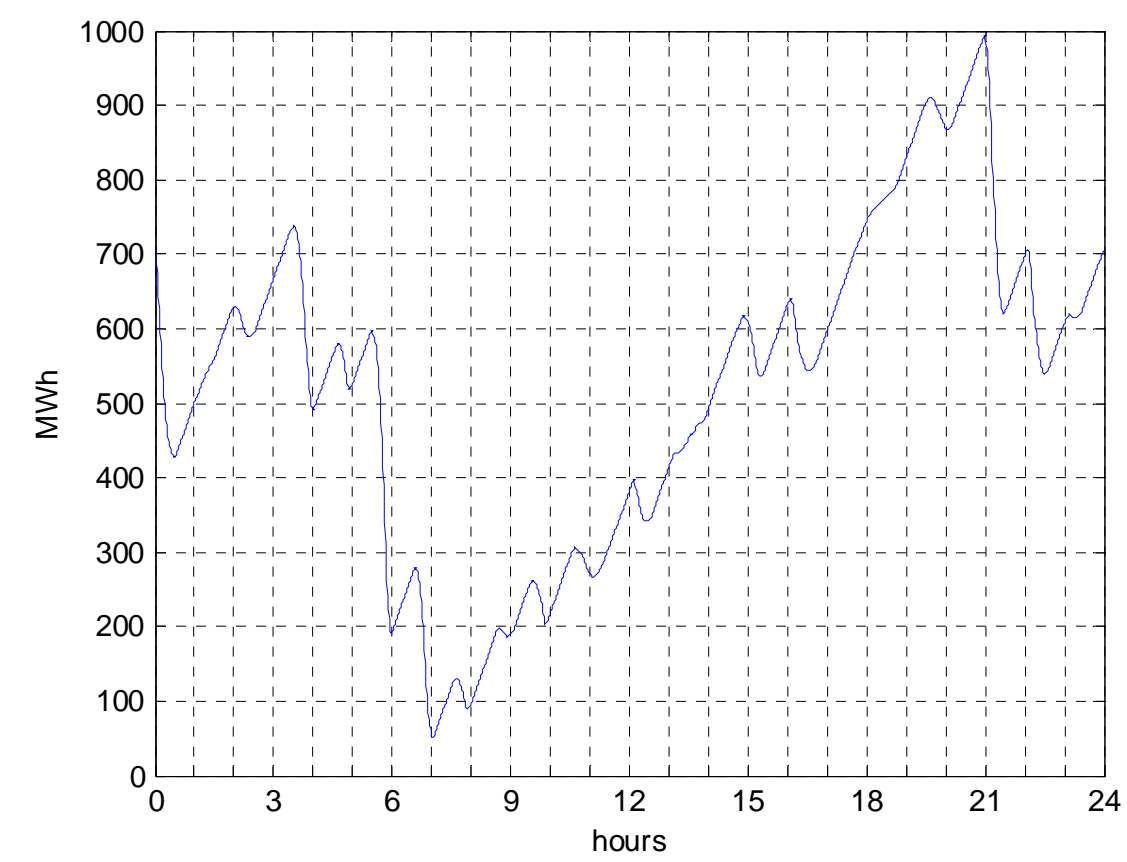

Figure B.13. Charging Status of Na-S Batteries, for Day 24, After the Complete Over-Generation Component is Curtailed by Wind Spillage

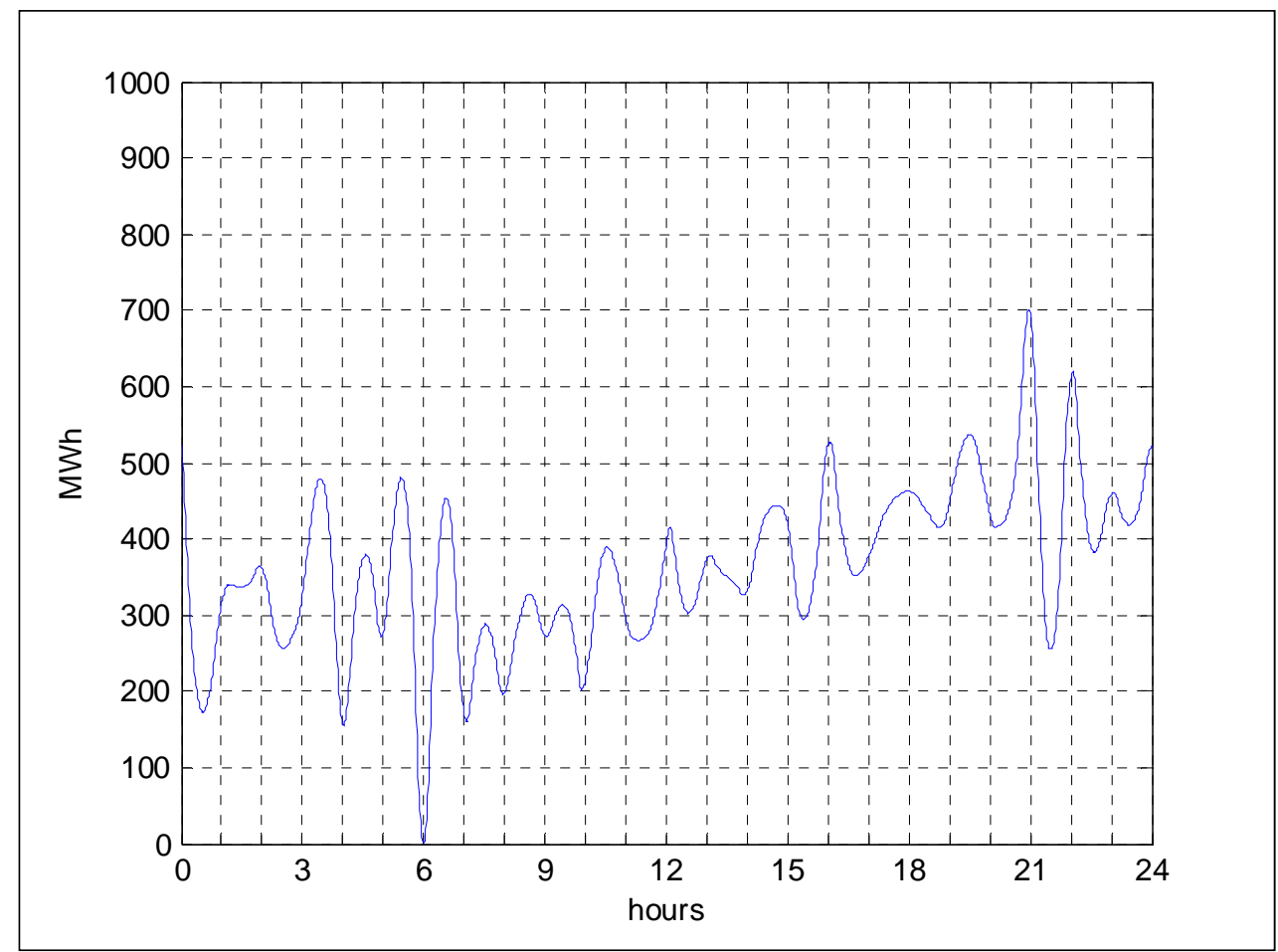

Figure B.14. Charging Status of Na-S Batteries Without Wind Spillage for Day 24 


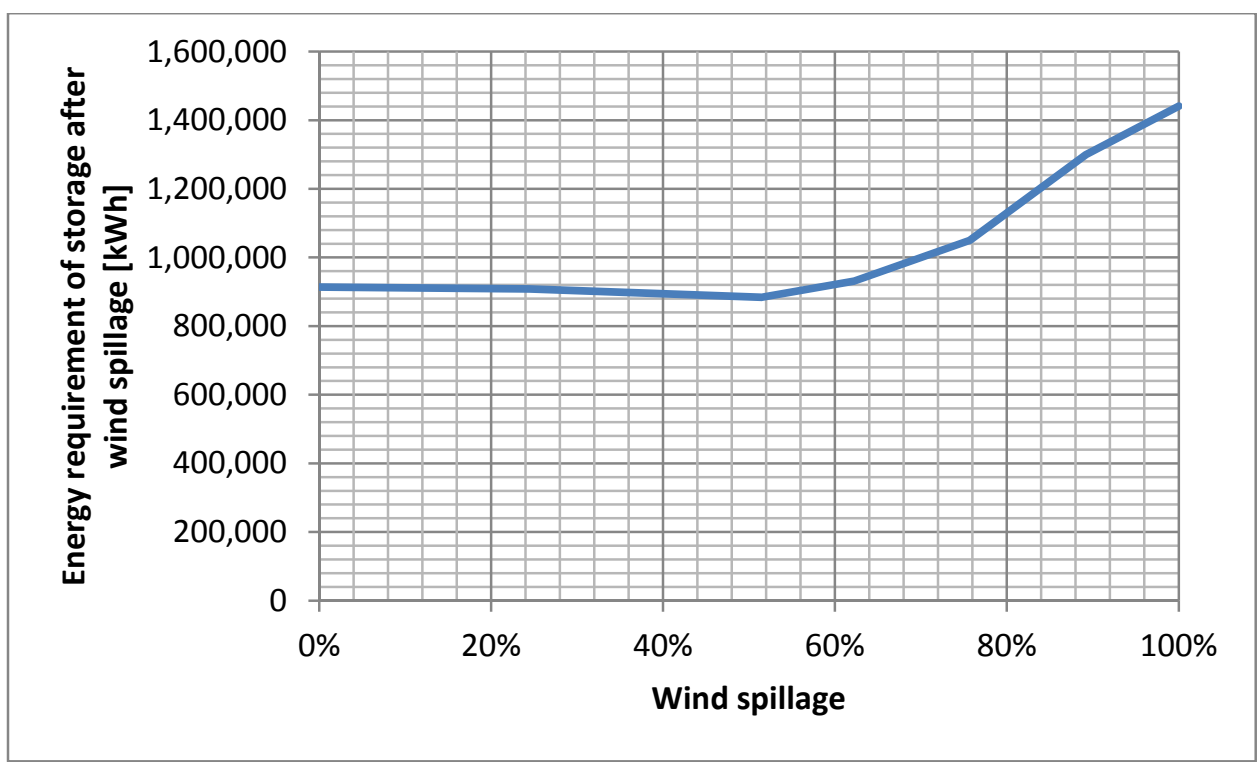

Figure B.15. Energy Requirements for Storage after Wind Spillage is Applied

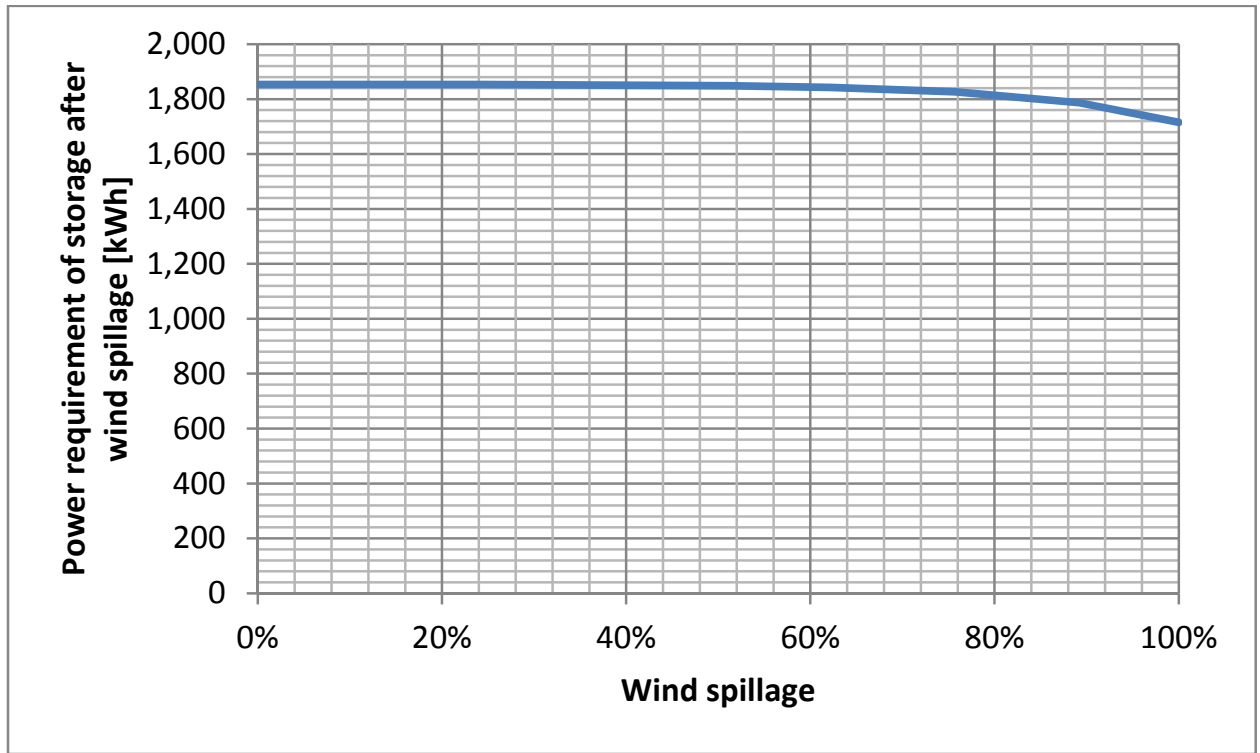

Figure B.16. Power Requirements for Storage After Wind Spillage is Applied 



\section{Appendix C}

Additional Balancing Requirements and Report Differentiators 



\section{Appendix C}

\section{Additional Balancing Requirements and Report Differentiators}

\section{C.1 Southwest (AZNM)}

The pattern of additional balancing signal determines the size of energy storage needed to provide the additional balancing services caused by future wind capacity and incremental load. Specifically, the magnitude of the signal determines the power capacity requirement of energy storage.

\section{C.1.1 Additional Balancing Requirements}

Monthly and daily balancing signals of region AZNM are shown in Figures C.1 and C.2 respectively. Based on the whole year simulation, the additional balancing power requirements are $331 \mathrm{MW}$ of inc. capacity and 449 MW of dec. capacity, using the BPA’s customary 99.5\% probability bound.

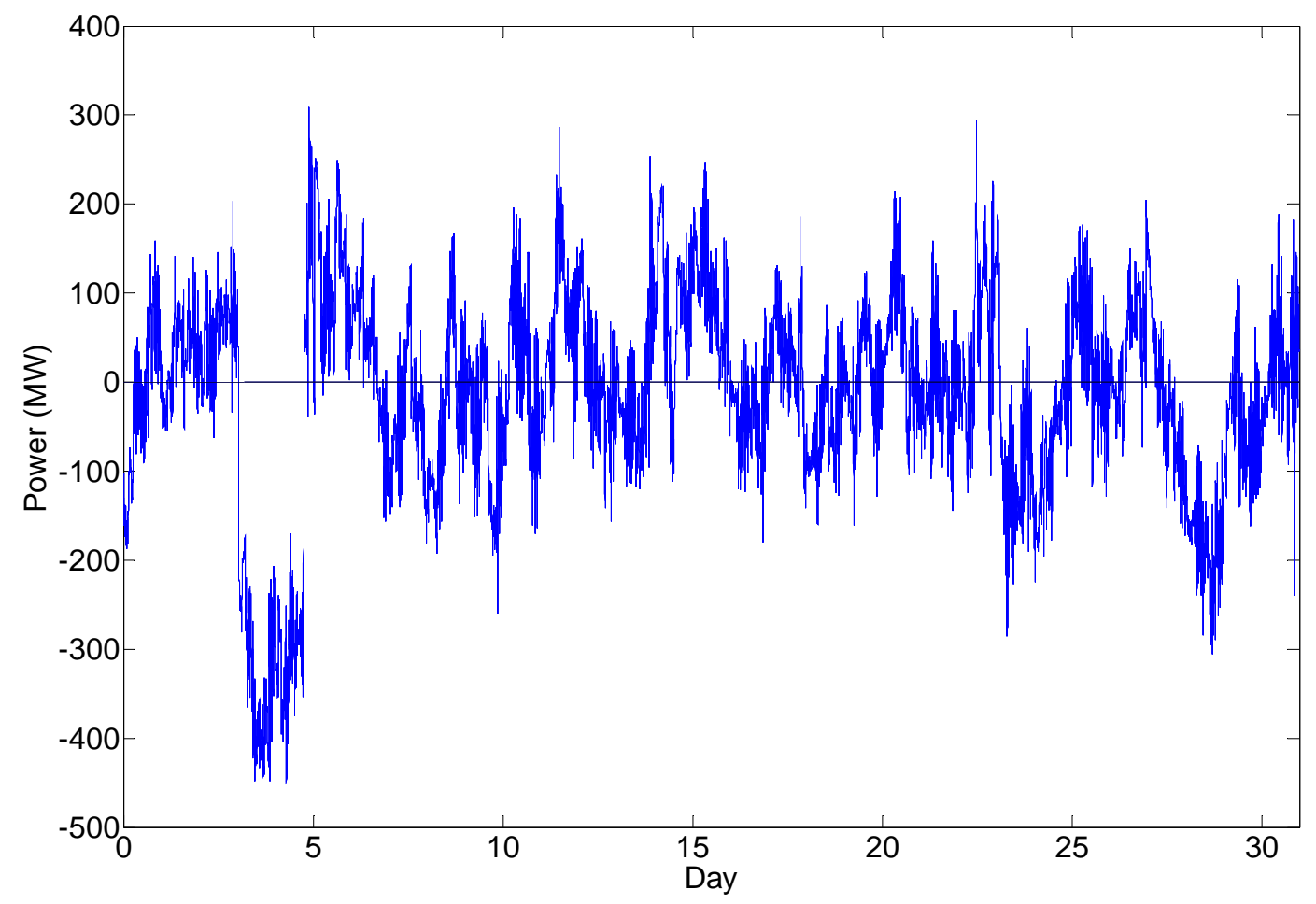

Figure C.1. One Month Additional Balancing Signal of August 2020 for AZNM 


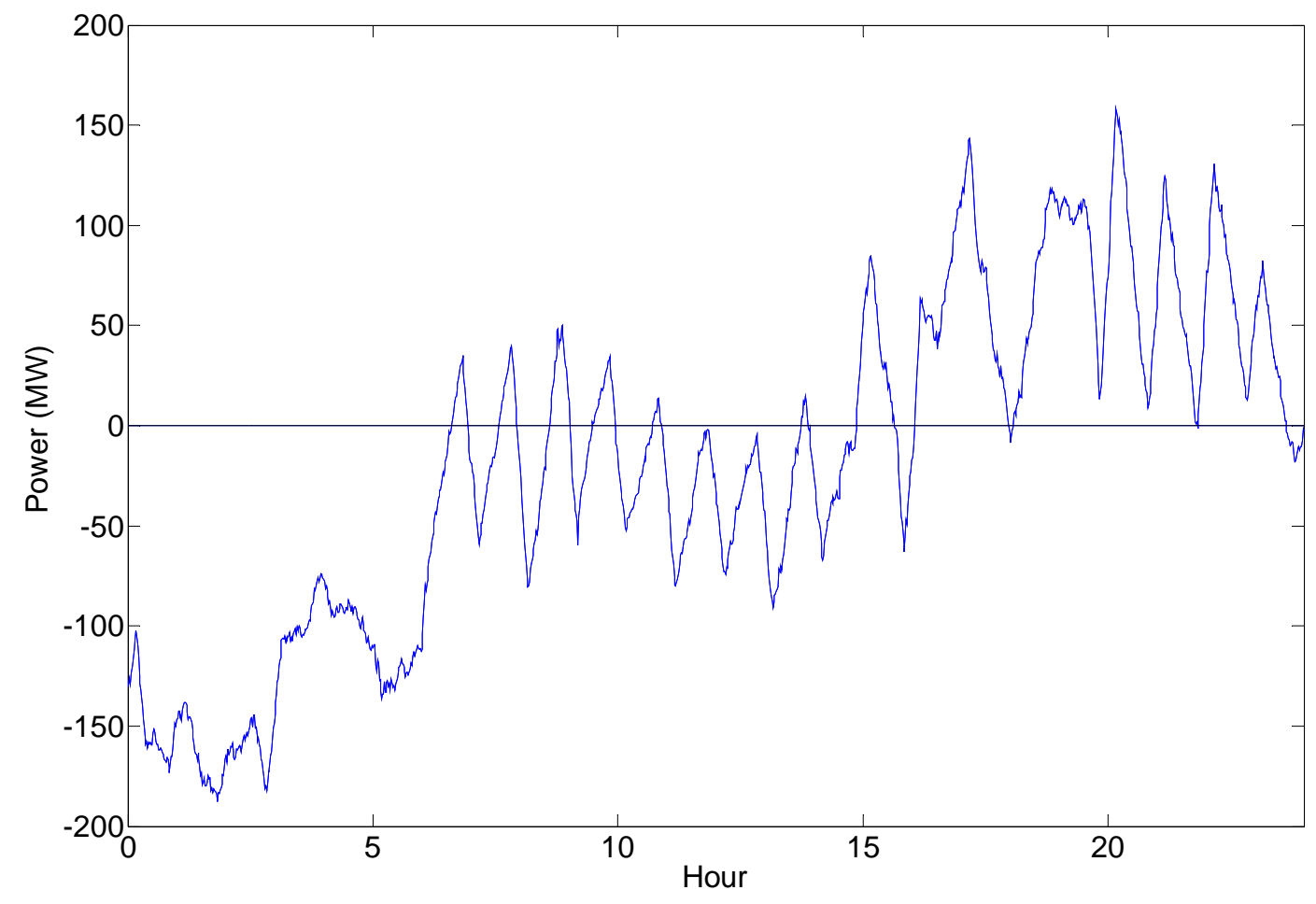

Figure C.2. One Typical Day Additional Balancing Signal of August 2020 for AZNM

\section{C.2 California (CAMX)}

The pattern of balancing signal determines the size of energy storage needed to provide the signal. Specifically, the magnitude of the signal determines the power capacity requirement of energy storage.

\section{C.2.1 Balancing Requirements}

Figure C.3 and C.4 shows monthly and daily balancing signal for CAMX, respectively. Based on the whole year simulation, the balancing power requirements are $972 \mathrm{MW}$ of inc. capacity and -999 MW of dec. capacity, using the BPA’s customary 99.5\% probability bound. 


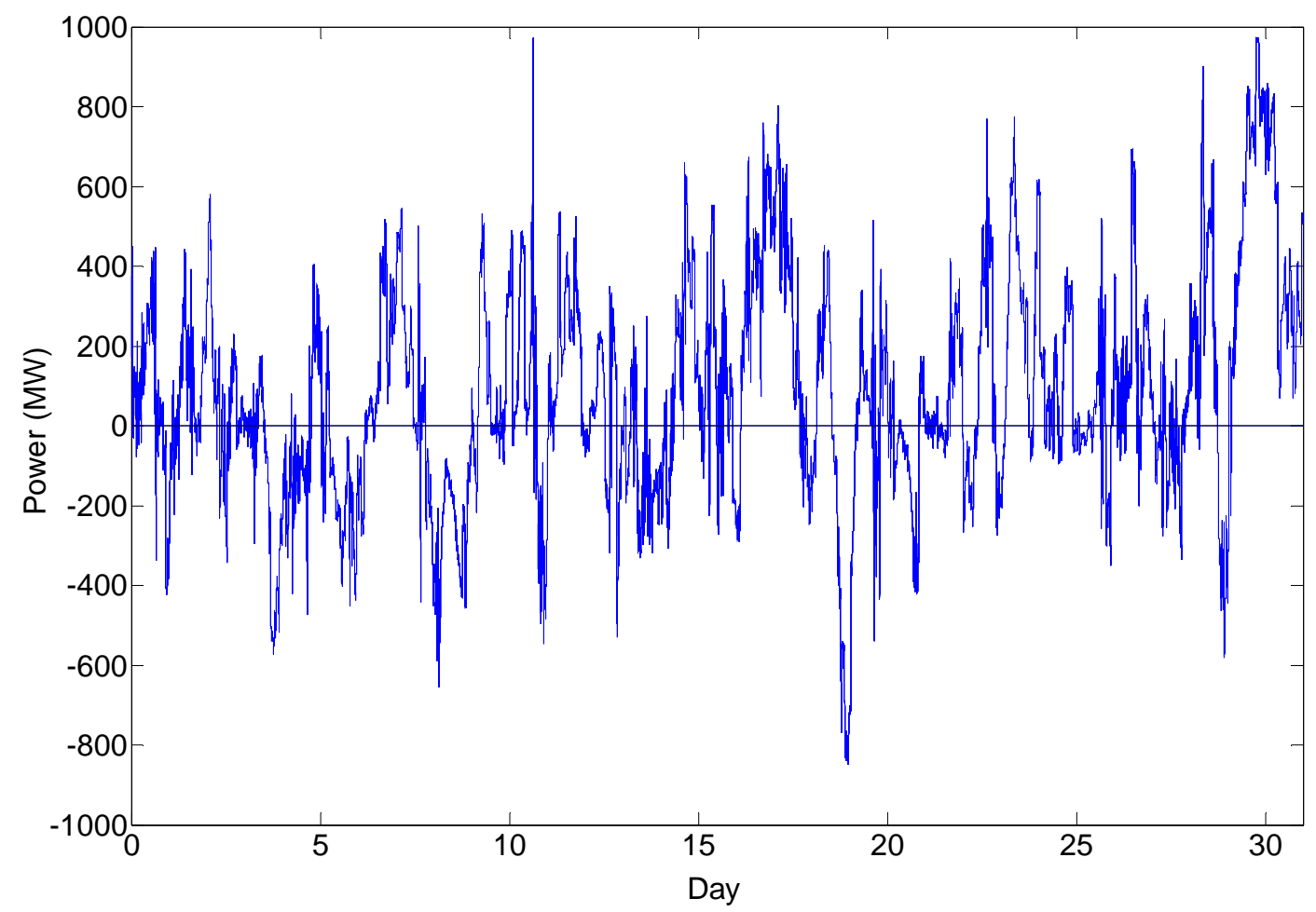

Figure C.3. One Month Additional Balancing Signal of August 2020

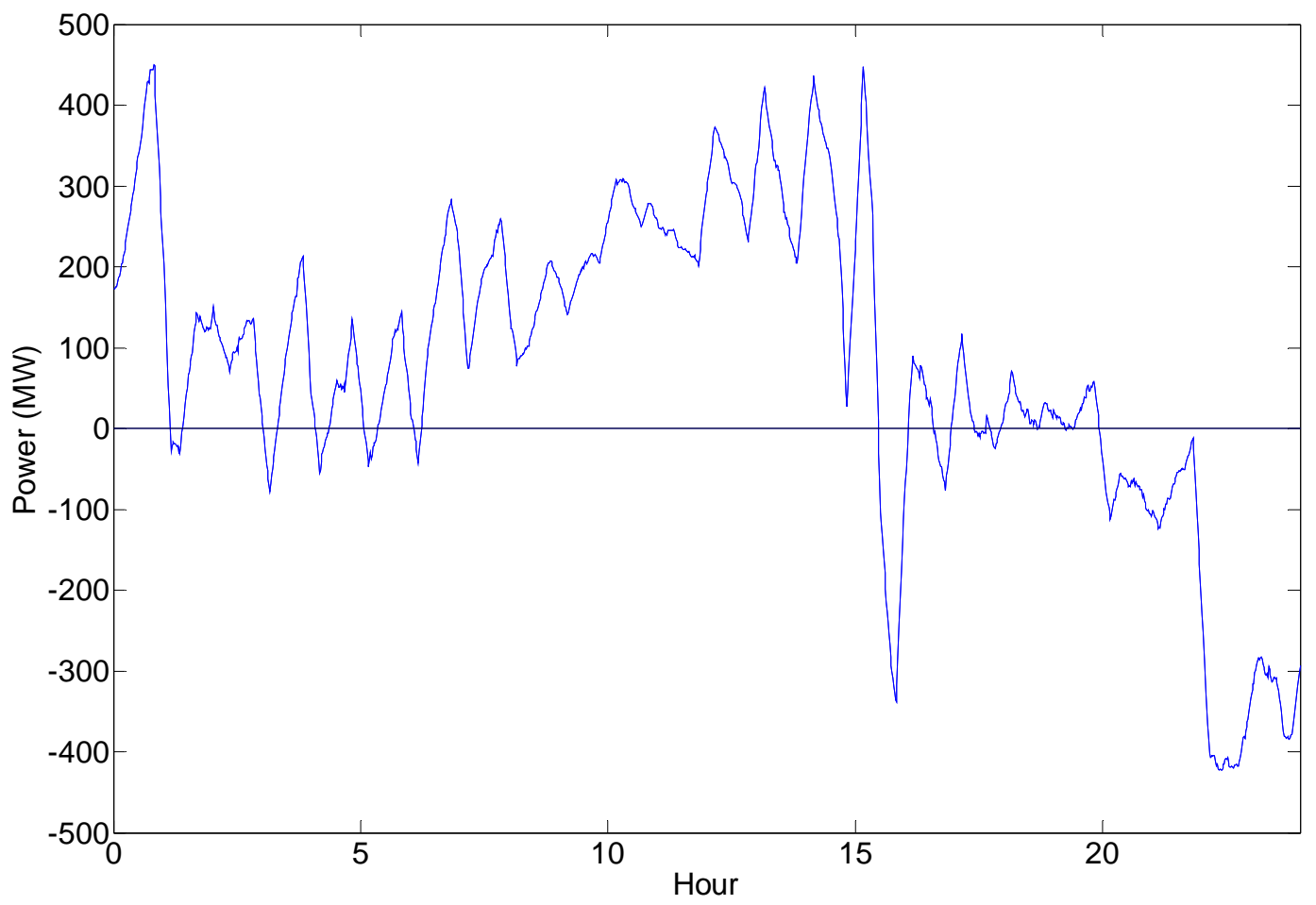

Figure C.4. One Typical Day Additional Balancing Signal of August 2020 


\section{C.3 Northwest Power Pool (NWPP)}

\section{C.3.1 Balancing Requirements}

Figure C.5 shows balancing signal for NWPP for the whole month of August. Figure C.6 displays the balancing signal for a day in August. Based on the whole year simulation, the balancing power requirements are $716 \mathrm{MW}$ of inc. capacity and -743 MW of dec. capacity, using the BPA's customary 99.5\% probability bound.

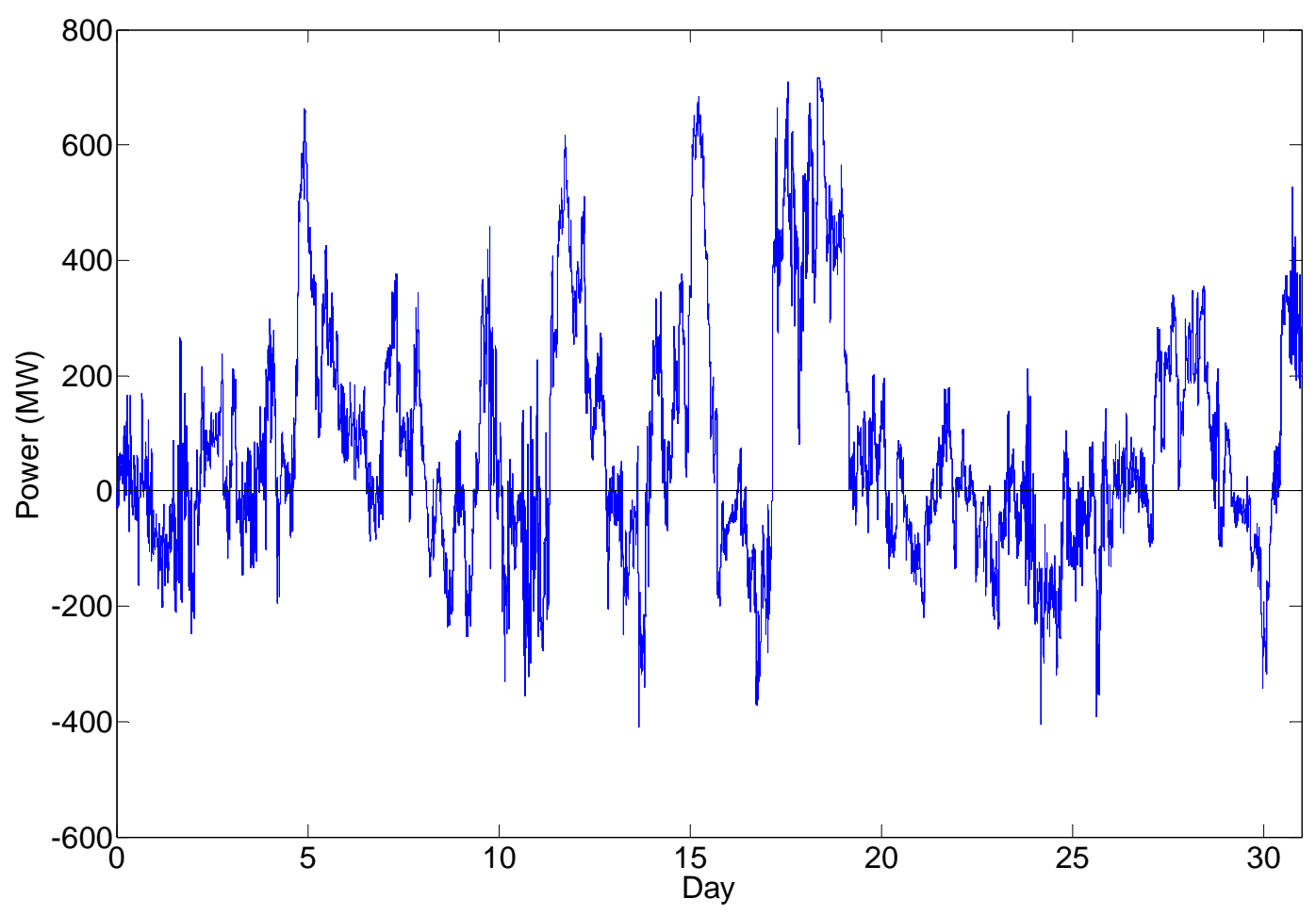

Figure C.5. One Month Additional Balancing Signal of August 2020 


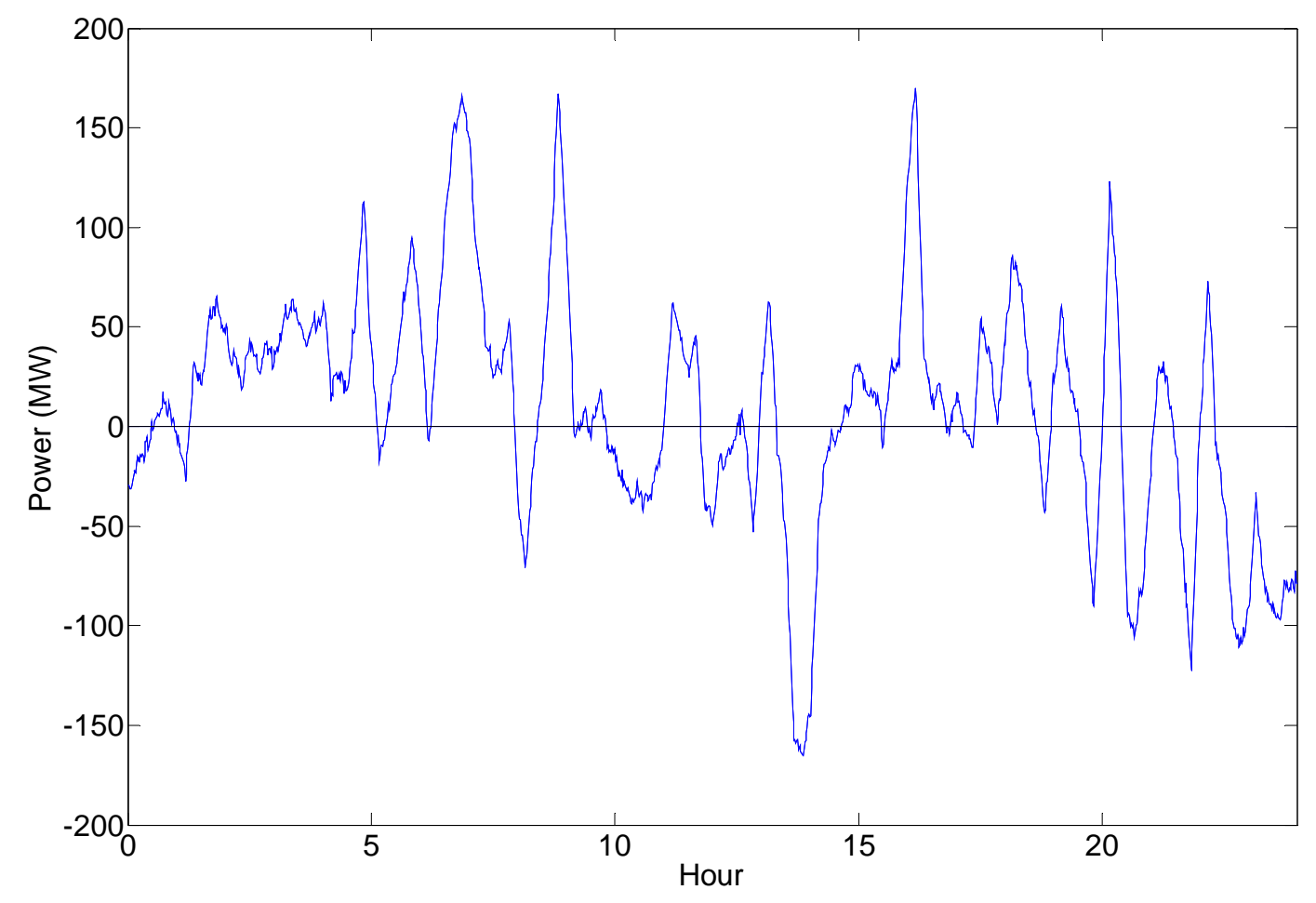

Figure C.6. One Typical Day Additional Balancing Signal of August 2020

\section{C.4 Rocky Mountain Power Area (RMPA)}

The pattern of balancing signal determines the size of energy storage needed to provide the signal. Specifically, the magnitude of the signal determines the power capacity requirement of energy storage.

\section{C.4.1 Balancing Requirements}

Figure C.7 and Figure C.8 show the balancing signal for the whole August and one day in August respectively. Based on the whole year simulation, the balancing power requirements are $1304 \mathrm{MW}$ of inc. capacity and -912 MW of dec. capacity, using the BPA’s customary 99.5\% probability bound. 


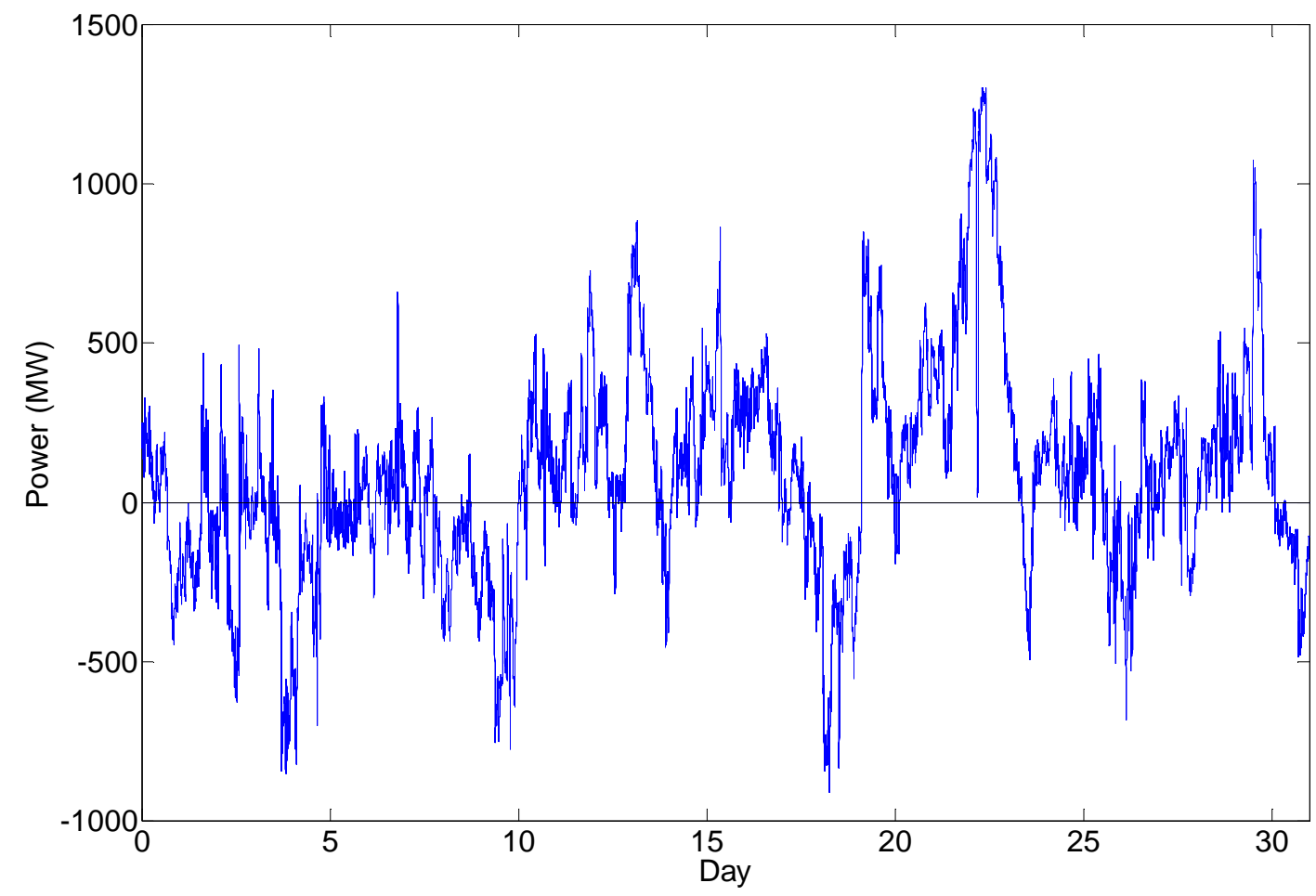

Figure C.7. One Month Additional Balancing Signal of August 2020

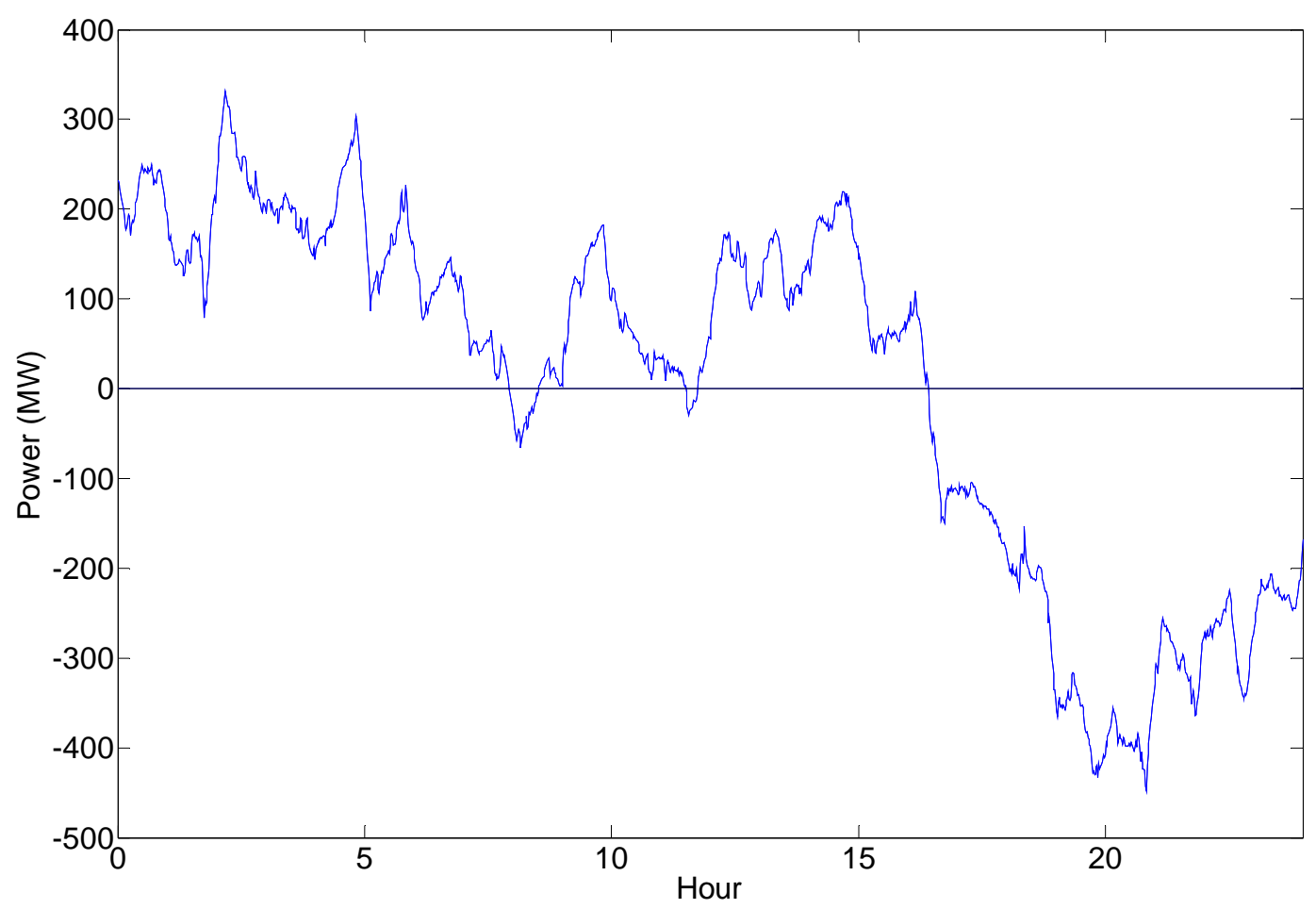

Figure C.8. One Typical Day Additional Balancing Signal of August 2020 
Appendix D

\section{Detailed Desciption of Energy Storage Analysis Landscape}





\section{Appendix D}

\section{Detailed Description of Energy Storage Analysis Landscape}

\section{Pacific Northwest National Laboratory}

The PNNL National assessment attempts to provide market sizes estimates for stationary energy storage system for two specific applications: balancing services and energy arbitrage. The Assessment is national in scope covering 22 regions of the U.S. power system. The market size estimate are based on engineering approaches that estimates the future balancing requirements based on the expected temporal and spatial variability from both wind generation and load demands. After estimating the market size for balancing services, the study analyzed for each region the lowest LCC technology that could provide the total balancing services for a 2020 scenario or the incremental services necessary as the grid expanse during the 2011 to 2020 time horizon. The cost calculations were based on a rigorous literature survey of today's cost and expected 2020 cost of nine different technologies (seven storage technologies, one combustion turbine, and one DR technology). Each technology was simulated as to how it would meet future estimated balancing requirements on a minute-by-minute basis. The analysis adopts a comprehensive and holistic approach by addressing capital costs, optimization of operating conditions such as DOD in terms of life vs. cost, operating and maintenance costs. Technology maturity levels were assigned to each storage technology to assess the risk to deployment. Cost information was obtained from extensive review of prior literature and phone calls with energy storage developers and component suppliers (such as electrode material, chemical cost, pumps).

Previous economic assessments of energy storage typically did not model the relationship between battery sizes and LCCs (none were found in the extensive literature review). The challenge in sizing storage cost-optimally (based on LCC) for a given application requires an optimization that solves the coupled problem associated with sizing (capital cost) and operation (cycle life, efficiency) of storage. The size of storage influences the DOD, with lower DOD corresponding to higher cycle life. In this analysis, the battery cycle life as a function of DOD was estimated from literature data. For each energy storage system considered in this analysis, a schedule of DODs and battery energy capacities was considered. This analysis provides insights into optimal sizing and the influence of oversizing a battery system on the total LCC.

The assessment considered hybrid systems to utilize the "sweet" spot of individual storage technologies such that each individual storage component contributes its strengths to the system to achieve a more cost-effective storage solution. Generally, a fast responding technology for high ramp rate resilience is combined with the slower responding technology that has a better cost effectiveness for storage of large amounts of energy. To explore the hybridization opportunity for balancing services, we established a cost-optimization approach, which considers technical operation and constraints, to identify the optimal pairing of two different storage technologies. Several of the two technologies pairings were investigated. 
Regarding energy arbitrage application, the analysis utilized PROMOD, a production cost model for economic dispatch of generators, widely used in the power industry. The economic viability of arbitrage opportunities for energy storage was evaluated and cost performance targets determined for storage to be cost-competitive. This part of the assessment required significant computing resources to explore the parameter space of different energy storage sizes and different locations in the WECC grid.

\section{Electric Power Research Institute}

The Electric Power Research Institute (EPRI) estimates the current market size for energy storage in the U.S. using a bottom-up approach considering storage requirement for each application (Rastler 2010). Bundled services with multiple revenue streams for storage were not considered. The EPRI studies (Rastler 2010, 2011a, 2012) estimated the price points at which energy storage may be economically viable for 17 applications. In the first two reports (2010, 2011a), long duration and short duration applications were considered, with PHES and CAES deemed appropriate for the former. The reports summarize the benefits for up to 17 different applications in terms of $\$ / \mathrm{kW}$, the power capacity potential in 10 years, and the financial market potential. EPRI estimated the benefits of energy storage over a period of 1 year. In some cases, several applications were grouped together, thus providing an upper bound on benefits. The market benefits for various applications along with their market size were provided. The cost in terms of $\$ / \mathrm{kW}$ and $\$ / \mathrm{kWh}$ for each technology was provided. In the 2012 presentation, sensitivity analysis was done by varying baseline parameters $\pm 10 \%$. Fixed replacement periods for various technologies were assigned. Detailed cost information for the power conditioning system were obtained from three vendors, while detailed electrical drawings for connection to the grid were used for estimation of transformer equipment and installation costs. These studies provided a rich set of construction cost data for installing MW-size energy storage systems. Projection of storage costs in the future was not done. Sizing of the batteries did not include effect of DOD and whether power or energy was the limiting factor for storage. Performance metrics such as efficiency, response time and ramp rate were not emphasized. The EPRI analysis takes into account the duration for energy storage, and assigns various storage life based on application and technology. One of the contrasting and complementary differences between the EPRI studies and the PNNL Assessment is that EPRI evaluated the incremental benefit that storage could provide given current grid conditions, while the PNNL approach assessed the future grid flexibility needs and then evaluated LCCs for a portfolio of technical storage and non-storage solutions. Both analyses complement each other. For compatible applications, the EPRI study reflects more a perspective of today's technology under today's competition, while the PNNL Assessment provides a perspective of the future competition.

\section{Midwest Independent System Operator}

The MISO energy storage study (Rastler 2011b) models energy storage as part of the capacity expansion process. This very recent study sought to determine economic potential for storage technologies in the MISO footprint. No detailed component cost analysis for individual storage technologies was provided. Technologies were characterized by a single incremental cost figure. EPRI's electric generation expansion analysis system (EGEAS) was used to explore the cost effectiveness of pumped hydro, CAES, and batteries. The study recognized that EGEAS has limitations in its temporal representation of the energy arbitrage opportunity to model the value of energy storage appropriately. PLEXOS, a production cost modeling environment was also used to provide more temporal granularity in the dispatch of energy storage system as part of a power system. However, the real-time energy market (5-min time increments) was not model in PLEXOS. The study only performed hourly economic 
dispatch simulating a day-ahead market. This significantly limited the valuation of energy storage with its superior ramping capability when following sharp fluctuations in the electricity generation from wind and solar energy technologies. However, this study provides valuable insights into the complexity of the long-term planning process as well as of the day-to-day operations process associate with the full utilization of the flexibility of energy storage. This study is unique in that it attempted to evaluate the value of energy storage not on the margin (i.e., what would be the benefits of one energy storage system in a certain utility footprint), but as part of the power system planning process that considers all options: transmission, generation, DR and energy storage.

\section{Sandia National Laboratories}

Sandia National Laboratories estimated market potential for energy storage in the U.S. in the year 2020 as part of a comprehensive report on stationary energy storage (Eyer 2010). The technical potential was defined as some portion of the total electric load for both the U.S. and California. More importantly, an estimate of the maximum storage market potential was provided in this study, that was based on the authors and other analysts' experiences with the industry. U.S. results were scaled up from California estimates. This maximum market potential for storage is listed for 19 different services for both California and the entire U.S. While the estimates were based on expert knowledge, and thus they were not model-based, they provide for the first time a detailed zero-order estimate on market sizes for 19 specific applications. The report also provides a very detailed discussion on the 26 distinct benefits that storage may furnish. The basis for the benefit estimates were general market value estimates that have been observed across the nation with some bounding minimum and maximum values. The PNNL Assessment differs from this Sandia study in both its approach and its scope. The Sandia report provided a very comprehensive zero-order market size estimate and a very detailed benefit assessment, however, it was based on expert knowledge and grid conditions that reflect historic trends and represent today's grid. The PNNL approach was model-based considering regional differences in terms of future wind capacity deployment and load growth projections. PNNL employed a stochastic model to estimate the balancing services for a 2020 grid and from those results estimated the market potential of energy storage. Rather than estimating benefits of energy storage for specific service, the PNNL Assessment attempted to answer the question: given the estimated need for additional balancing services for a 2020 grid scenario, what storage technologies and competing alternatives (i.e., combustion turbine and DR) are likely to be the most cost-effective technology? This approach provides different insights into the competitiveness and role of energy storage compared to the Sandia study and thus complements the existing literature.

\section{Southern California Edison}

The 2010 SCE study (Ritterhausen 2010) published a comprehensive analysis of cost/benefits of energy storage for various applications in the SCE service territory. The U.S. energy storage market size studies from various reports was reviewed. The study is unique in that it assessed the value of storage at various topological locations within SCE's grid ranging from the site of the generation resource, within transmission system, as well as within distribution system down to the end user site. This analysis studied 12 applications or use-cases which incorporated one or more of the 22 operational uses. The benefit/cost ratio for a few select applications was calculated, with what-if 2020 scenarios for falling technology costs and higher renewable penetration. Their study assigned the largest benefit/cost ratio for applications that deferred or displaced peak-capacity costs over several hours. The effect of diminishing returns on energy shifting was estimated. The model included installation costs and investment tax credit. This study contributed to the public literature by its comprehensiveness in both the locational aspect of energy 
storage (central plant versus distributed) as well as in the selection and definition of multiple services for energy storage. The cost/benefit analysis was performed for the SCE footprint, which renders the study to be most likely very accurate in the value estimates compared to other national studies, while at the same time, it limits the findings to the SCE grid, with potential applications to California as a whole.

\section{DNV Keuring Electrotechnisch Materieel Arnhem}

DNV Keuring Electrotechnisch Materieel Arnhem (DNV KEMA) has a long history in evaluating and testing energy storage system for grid applications. One of the recent studies evaluated the storage value for the CAISO grid (KEMA 2010). The effect of renewable penetration of $20 \%$ and $33 \%$ on CAISO was estimated using KEMA's KERMIT model. The energy storage market size for regulation/ ramping services in the 33\% RPS scenario was estimated using highly detailed operations data from the CAISO. The model performs a dynamic simulation of the grid taking into account existing generators to assess system performance in the second-hour time frame. The model was calibrated using existing generator fleet, daily schedules, loads, interchange, ACE and frequency data in the 4-second and 1-minute interval, extending the model to 2012 and 2020 RPS levels of $20 \%$ and 33\%, respectively. Control algorithms for storage were developed, and the benefits of storage estimated for the $20 \%$ and 33\% RPS scenarios. The faster ramp rate of storage was found to be beneficial over gas turbines. Avoided greenhouse gas emissions using storage was also estimated. This study provided deep insights on operational improvements when using fast responding energy storage technologies under realistic grid conditions.

\section{National Renewable Energy Laboratory}

The National Renewable Energy Laboratory (NREL) studied the role of energy storage for renewables integration (Sullivan 2008; Denholm 2010). In the 2008 study, three types of energy storage technologies were evaluated as part of an expansion planning process using the ReEDS model. This study looked at the economics of energy storage as part of long-term planning process to estimate generation capacity additions for the 2050 time horizon. Because of the modeling framework of ReEDS, the capacity value and the energy value of storage could be modeled. Ancillary services and transmission flexibility to meet ramping requirement were outside the scope of this analysis.

The 2010 NREL study focused on the role of energy storage with renewable electricity generation. Rather than focusing exclusively on energy storage, the study frame the problem as a renewable energy technology integration cost issue. As the fraction of wind and solar energy technology increase, grid operators need more grid flexibility to accommodate the variability from the renewable resources. Several competing technologies are available. Energy storage is one of several technology options. This study suggested a flexibility supply curve, in which energy storage may be at the higher cost segment of the supply curve. Other potentially lower-cost strategies are supply and reserve sharing, flexible generation, flexible demand, new loads, and renewable curtailment. This study provided a different perspective on the energy storage analyses by analyzing the ultimate operational needs at growing variable renewable energy resources. How these needs are met will be determined by economics.

\section{Hybrid Storage Studies}

Several researchers have studied hybrid storage systems. This research has largely focused on purely operational aspects of hybrid storage systems. These studies discussed strategies of how to optimize the 
operation of systems given a certain size of two storage components. Only two papers have focused on economic sizing of a hybrid storage - Henson (2008) and Vosen (1999). One work showed only the concept of a very simplified model without consideration of balancing services and applied to one single pair of technologies, while the other work was applied to a small power system at a particular location, also considering a single pair of storage technologies. The optimization considers technical aspects, such as ramp rate limitations and storage life degradation, combined with economic aspects, such as LCC influenced by oversizing storage technologies. In other words, the optimization is a coupled sizing and operations problem.

Henson illustrated the potential of hybrid energy storage system to minimize system LCCs; this reference used a simplified model for the need for balancing, and only considered a single pair of storage technologies. An optimization of a hybrid storage system is presented in part of the analysis in Vosen (1999), where a combination of short-term and long-term storage is optimized for seasonal and daily energy storage to obtain minimal cost design. However, the provision of balancing services is not considered and the hybrid storage system is applied in a small power system in one specific location. Other references such as Lemofouet (2006) and Lukic (2006) focus only on the operational aspects of hybrid storage systems. 


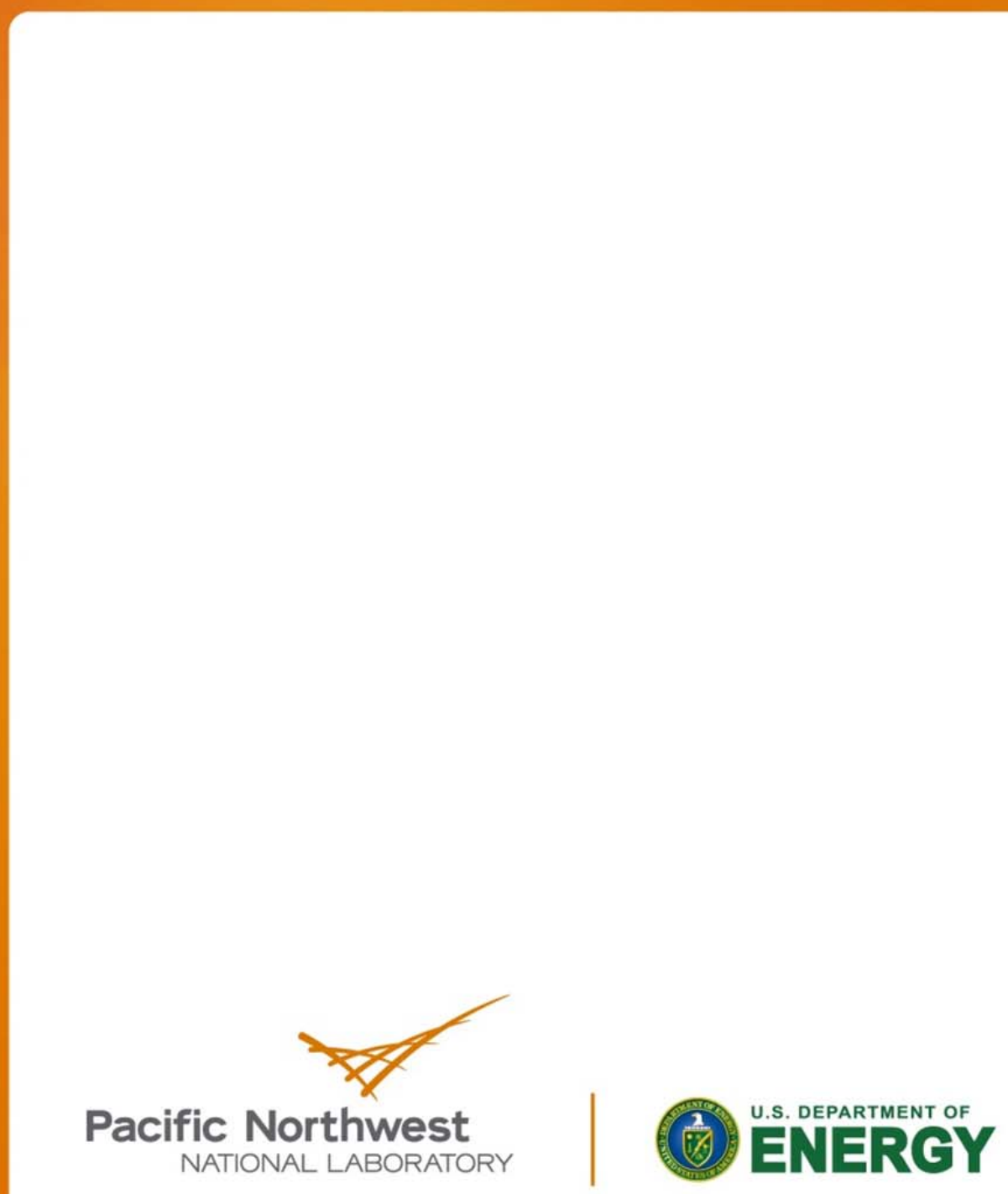

Proudly Operated by Battelle Since 1965

902 Battelle Boulevard

P.O. Box 999

Richland, WA 99352

1-888-375-PNNL (7665)

www.pnnl.gov 